\title{
Synthesis of Chiral 3-Substituted Indanones via an Enantioselective Reductive-Heck Reaction
}

\author{
Ana Minatti, Xiaolai Zheng, Stephen L. Buchwald* \\ Department of Chemistry \\ Massachusetts Institute of Technology \\ Cambridge, MA 02139 \\ sbuchwal@mit.edu
}

\section{Supporting Information}

Table of Contents:

$\begin{array}{ll}\text { General Considerations } & \text { S2 }\end{array}$

$\begin{array}{ll}\text { Synthesis of }(E)-2 \text { '-iodo-chalcone and }(E)-2 \text { '-bromo-chalcone } & \text { S3 }\end{array}$

Experimental procedures for the synthesis of $(E)-2^{\prime}$-hydroxy- $\alpha, \beta$-unsaturated ketones S3

Experimental procedures for the synthesis of nonaflates and triflates 1a-m $\quad$ S8

$\begin{array}{ll}\text { Experimental procedures for the synthesis of compounds 2a-m } & \text { S14 }\end{array}$

Experimental procedures for the synthesis of compounds 3a-k and $4 \quad$ S20

$\begin{array}{ll}\text { Experimental procedure for the synthesis of Pd-complex I } & \text { S25 }\end{array}$

$\begin{array}{ll}\text { Experimental procedure for the synthesis of compounds } \mathbf{5} \text { and } \text { cis-1m } & \text { S26 }\end{array}$

Crystallographic information and ORTEP file for Pd-complex I $\quad$ S28

$\begin{array}{ll}\text { NMR spectra } & \text { S49 }\end{array}$

$\begin{array}{ll}\text { References } & \text { S107 }\end{array}$ 


\section{General Considerations.}

All reactions were carried out under an argon atmosphere in oven-dried glassware. $\operatorname{Pd}(\mathrm{OAc})_{2}$ was a generous gift from BASF and used without further purification. $\operatorname{Pd}(\mathrm{dba})_{2}$ was purchased from Strem and used without further purification. ( $R$-MeOBIPHEP, $(R)$-3,5-XylMeOBIPHEP and $(R)-3,5-t$-BuylMeOBIPHEP were provided by Dr. Rudolph Schmid (Hoffmann-LaRoche). $(R)$-BINAP, $(R)$-TolBINAP and $(R)$-3,5-XylBINAP were purchased from Strem. Proton sponge was purchased from Lancaster. 1,2,2,6,6-pentamethylpiperidine was purchased from Fluka and stored under nitrogen. All other reagents were purchased from commercial sources and used without further purification. Dichloromethane, toluene, diethyl ether and tetrahydrofuran were purchased from J. T. Baker in CYCLE-TAINER solvent delivery kegs, which were vigorously purged with argon for $2 \mathrm{~h}$, and further purified by passing the solvent through two packed columns of neutral alumina and copper(II) oxide under argon pressure. Anhydrous 1,4-dioxane and DMF were purchased from Aldrich in Sure-Seal ${ }^{\circledR}$ bottles.

Yields reported for the preparation of starting materials refer to a single experiment whereas those reported in Tables 2 and 3 are an average of at least two independent runs. Compounds described in the literature were characterized by comparing their ${ }^{1} \mathrm{H}$ NMR, IR spectra and melting point ( $\mathrm{mp}$ ) to the previously reported data.

Column chromatography was performed employing EM Science silica gel 60 (230-400 mesh). GC analyses were performed on an Agilent 6890 instrument with an FID detector and a Agilent $10 \mathrm{~m} \times 0.1 \mathrm{~mm}$ DB-1 capillary column using dodecane as an internal standard. Analytic high performance liquid chromatography (HPLC) was performed on a Hewlett-Packard 1100 Series HPLC equipped with a variable wavelength detector using the corresponding chiral stationary phase.

Elemental analyses were performed by Atlantic Microlab, Inc., Norcross, GA. ${ }^{1} \mathrm{H}$ NMR, ${ }^{13} \mathrm{C}$ NMR, ${ }^{31} \mathrm{P}$ NMR and ${ }^{19} \mathrm{~F}$ NMR spectra were recorded on Bruker Advance $400 \mathrm{MHz}$ instruments or on a Varian $500 \mathrm{MHz}$ instrument with chemical shifts reported in ppm relative to the deuterated solvent or the internal standard tetramethylsilane. IR spectra were recorded on a Perkin-Elmer System 2000 FT-IR instrument ( $\mathrm{KBr}$ disc). Melting points (uncorrected) were obtained on a Mel-Temp II capillary melting point apparatus. Optical rotations were measured on a Perkin-Elmer Model 241 Polarimeter at $\mathrm{T}=21^{\circ} \mathrm{C}$. 


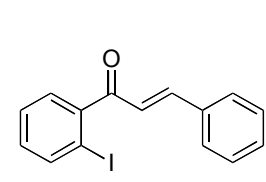

\section{(E)-2'-Iodo-chalcone 1a $(X=I)^{\mathrm{i}}$}

A solution of $\mathrm{NaOH}(2.5 \mathrm{~g}, 63 \mathrm{mmol})$ in water $(15 \mathrm{~mL})$ and ethanol $(15 \mathrm{~mL})$ was cooled to 0

${ }^{\circ} \mathrm{C}$ and 2'-iodoacetophenone $(6.0 \mathrm{~mL}, 42 \mathrm{mmol})$ and benzaldehyde $(4.3 \mathrm{~mL}, 42 \mathrm{mmol})$ were slowly added successively. The reaction mixture was allowed to warm to room temperature over night. After $12 \mathrm{~h}$ the reaction mixture was diluted with diethyl ether $(400 \mathrm{~mL})$, the organic phase was separated and washed with a saturated, aqueous solution of $\mathrm{NH}_{4} \mathrm{Cl}$ and dried over $\mathrm{MgSO}_{4}$. The solvent was evaporated and the crude material was purified by column chromatography (hexane $/ \mathrm{CH}_{2} \mathrm{Cl}_{2} 1: 1$ ) to yield the desired product as a pale yellow oil (13.3 g, 95\%). ${ }^{1} \mathrm{H}$ NMR (400 MHz, $\left.\mathrm{CDCl}_{3}\right) \delta 7.89$ (dd, $\left.1 \mathrm{H}, J=8.0 \mathrm{~Hz}, 0.8 \mathrm{~Hz}\right), 7.51(\mathrm{~m}, 2 \mathrm{H}), 7.36-7.44(\mathrm{~m}, 6 \mathrm{H})$, $7.13(\mathrm{~m}, 1 \mathrm{H}), 7.07$ (d, 1H, $J=16.0 \mathrm{~Hz}) ;{ }^{13} \mathrm{C} \mathrm{NMR}\left(100 \mathrm{MHz}, \mathrm{CDCl}_{3}\right) \delta 195.7,146.8,144.4,139.9,134.2,131.3$, 130.9, 128.9, 128.5, 128.4, 127.9, 125.4, 92.2; IR (neat) v3059, 1959, 1645, 1604, 1495, 1449, 1425, 1331, 1286, 1211, 1097, 1061, 1013, 978, 872, 753, 729, 702, 598, 568, 485, $446 \mathrm{~cm}^{-1}$; Anal. Calcd. for $\mathrm{C}_{15} \mathrm{H}_{11} \mathrm{IO}$ : C, 53.92; H, 3.32. Found: C, 54.00; H, 3.32.

\section{(E)-2'-Bromo-chalcone 1a $(\mathrm{X}=\mathrm{Br})^{\mathrm{ii}}$}

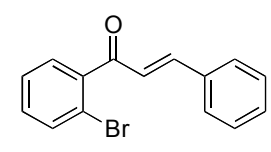

A solution of $\mathrm{NaOH}(1.9 \mathrm{~g}, 47 \mathrm{mmol})$ in water $(10 \mathrm{~mL})$ and ethanol $(10 \mathrm{~mL})$ was cooled to 0

${ }^{\circ} \mathrm{C}$ and 2'-bromoacetophenone $(4.2 \mathrm{~mL}, 31 \mathrm{mmol})$ and benzaldehyde $(3.2 \mathrm{~mL}, 31 \mathrm{mmol})$ were slowly added successively. The reaction mixture was allowed to warm to room temperature over night. After $12 \mathrm{~h}$ the reaction mixture was diluted with diethyl ether $(400 \mathrm{~mL})$, the organic phase was separated and washed with a saturated, aqueous solution of $\mathrm{NH}_{4} \mathrm{Cl}$ and dried over $\mathrm{MgSO}_{4}$. The solvent was evaporated and the crude material was purified by column chromatography (hexane $/ \mathrm{CH}_{2} \mathrm{Cl}_{2} 1: 1$ ) to yield the desired product as a pale yellow oil (8.56 g, 95\%). ${ }^{1} \mathrm{H}$ NMR (400 MHz, $\left.\mathrm{CDCl}_{3}\right) \delta 7.63(\mathrm{~d}, 1 \mathrm{H}, J=8.0 \mathrm{~Hz}), 7.54(\mathrm{~m}, 2 \mathrm{H}), 7.45(\mathrm{~d}, 1 \mathrm{H}, J=16.0 \mathrm{~Hz})$, 7.37-7.45 (m, 4H), $7.32(\mathrm{dd}, 1 \mathrm{H}, J=2.0,7.8 \mathrm{~Hz}), 7.11(\mathrm{~d}, 1 \mathrm{H}, J=16.0 \mathrm{~Hz}) ;{ }^{13} \mathrm{C} \mathrm{NMR}\left(100 \mathrm{MHz}, \mathrm{CDCl}_{3}\right) \delta$ 194.6, 146.6, 141.0, 134.3, 133.4, 131.4, 130.9, 129.1, 129.0, 128.6, 127.4, 126.0, 119.4; IR (neat) v3060, 1959, 1649, 1606, 1495, 1449, 1429, 1332, 1299, 1211, 1102, 1065, 1027, 978, 872, 754, 704, 599, 568, 486, $456 \mathrm{~cm}^{-1}$; Anal. Calcd. for $\mathrm{C}_{15} \mathrm{H}_{11} \mathrm{BrO}$ : C, 62.74; H, 3.86. Found: C, 63.00; H, 3.88.

\section{General procedure for the synthesis of $(E)$-2'-hydroxy- $\alpha, \beta$-unsaturated ketones.}

A solution of $\mathrm{NaOH}$ (10 equiv) in water ( $3 \mathrm{~mL} / \mathrm{mmol}$ ketone) was added to a solution of the corresponding 2'hydroxy-acetophenone ( 1 equiv) in $\mathrm{MeOH}$ or $\mathrm{EtOH}(3 \mathrm{~mL} / \mathrm{mmol}$ ketone). The obtained solution was cooled to 0 ${ }^{\circ} \mathrm{C}$ in an ice bath and the corresponding aldehyde (1-1.2 equiv) was slowly added. The reaction mixture was allowed to warm to room temperature and stirred for $20 \mathrm{~h}$. The reaction mixture was poured into a mixture of ice and $\mathrm{HCl}(12 \mathrm{M})$ to adjust the $\mathrm{pH}$ of the solution to 2. Workup A: The obtained solid was filtered off and dissolved in $\mathrm{CH} 2 \mathrm{Cl}$ 2. The organic phase was washed with brine and dried over $\mathrm{MgSO}_{4}$. The solvent was evaporated under 
reduced pressure and the crude material was further purified by recrystallization from the corresponding solvent or by column chromatography on silica gel. Workup $\mathrm{B}: \mathrm{CH}_{2} \mathrm{Cl}_{2}$ was added to the obtained solution, the organic phase was separated, washed with brine and dried over $\mathrm{MgSO}_{4}$. The solvent was evaporated under reduced pressure and the crude material was purified by column chromatography on silica gel.

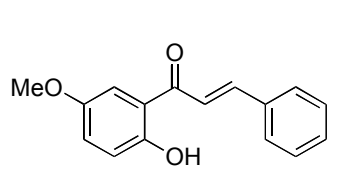

(E)-2'-Hydroxy-5'methoxy-chalcone SM-1 ${ }^{\text {iii }}$

(E)-2'-Hydroxy-5'methoxy-chalcone was prepared according to the general procedure using 2'-hydroxy-5' -methoxy-acetophenone (3.3 g, $0.020 \mathrm{~mol})$, benzaldehyde (2.4 mL, 24 $\mathrm{mmol})$ and $\mathrm{NaOH}(8.0 \mathrm{~g}, 0.20 \mathrm{~mol})$ in $\mathrm{MeOH} / \mathrm{H}_{2} \mathrm{O}$. Workup $\mathrm{B}$, followed by purification via column chromatography (hexane/ethyl acetate 5:1) provided the title compound as a viscous orange oil (3.25 g, 64\%). ${ }^{1} \mathrm{H}$ NMR (400 MHz, $\left.\mathrm{CDCl}_{3}\right) \delta 12.42(\mathrm{~s}, 1 \mathrm{H}), 7.83(\mathrm{~d}, 1 \mathrm{H}, J=15.5 \mathrm{~Hz}), 7.58(\mathrm{~m}, 2 \mathrm{H}), 7.50(\mathrm{~d}, 1 \mathrm{H}, J=15.5 \mathrm{~Hz}), 7.37$ $(\mathrm{m}, 3 \mathrm{H}), 7.28(\mathrm{~d}, 1 \mathrm{H}, J=3.0 \mathrm{~Hz}), 7.07(\mathrm{dd}, 1 \mathrm{H}, J=3.0,9.0 \mathrm{~Hz}), 6.91(\mathrm{~d}, 1 \mathrm{H}, J=9.1 \mathrm{~Hz}), 3.77(\mathrm{~s}, 3 \mathrm{H}) ;{ }^{13} \mathrm{C} \mathrm{NMR}$ $\left(100 \mathrm{MHz}, \mathrm{CDCl}_{3}\right) \delta 193.3,157.9,151.7,145.6,134.5,131.0,129.1,128.7,123.9,120.0,119.6,119.3,112.8$, 56.0; IR (neat) $v 2939,2835,1644,1575,1487,1355,1266,1174,1044,784,694,581 \mathrm{~cm}^{-1}$; Anal. Calcd. for $\mathrm{C}_{16} \mathrm{H}_{14} \mathrm{O}_{3}$ : C, 75.57; H, 5.55. Found: C, 75.49; H, 5.61.

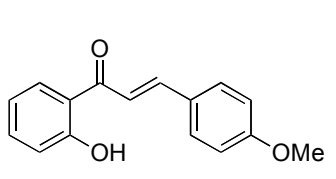

\section{(E)-2'-Hydroxy-4-methoxychalcone SM-2 ${ }^{\text {iv }}$}

(E)-2'-Hydroxy-4-methoxychalcone was prepared according to the general procedure using 2'-hydroxy-acetophenone (6.0 mL, $0.050 \mathrm{~mol}), p$-anisaldehyde (7.3 mL, $0.060 \mathrm{~mol})$ and $\mathrm{NaOH}(20.0 \mathrm{~g}, 0.500 \mathrm{~mol})$ in $\mathrm{MeOH} / \mathrm{H}_{2} \mathrm{O}$. Workup A, followed by recrystallisation from EtOH provided the title compound as a yellow solid (6.9 g, 54\%). ${ }^{1} \mathrm{H}$ NMR (400 MHz, $\left.\mathrm{CDCl}_{3}\right) \delta 13.00(\mathrm{~s}, 1 \mathrm{H}), 7.90-7.95(\mathrm{~m}, 2 \mathrm{H})$, 7.63-7.67 (m, 2H), 7.56 (d, 1H, J=15.4 Hz), 7.49-7.53 (m, 1H), 7.04 (d, 1H, J=8.4 Hz), 6.94-6.99 (m, 3H), 3.88 (s, 3H); IR (neat) $v 3073,3000,2960,1565,1511,1487,1439,1366,1342,1304,1269,1200,1175,1034,988$, 860, 821, 803, 763, 749, 565, $512 \mathrm{~cm}^{-1}$; Anal. Calcd. for $\mathrm{C}_{16} \mathrm{H}_{14} \mathrm{O}_{3}$ : C, 75.57; H, 5.55. Found: C, 75.82; H, 5.56; mp 88-90 ${ }^{\circ} \mathrm{C}\left(\right.$ lit. $\left.{ }^{4 a} \mathrm{mp} 88-90{ }^{\circ} \mathrm{C}\right)$.

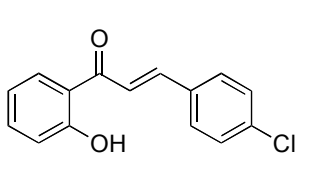

\section{(E)-2'-Hydroxy-4-chlorochalcone SM-3 ${ }^{\mathrm{v}}$}

(E)-2'-Hydroxy-4-chlorochalcone was prepared according to the general procedure using 2'hydroxy-acetophenone $(2.4 \mathrm{~mL}, 0.020 \mathrm{~mol}), p$-chlorobenzaldehyde $(3.4 \mathrm{~mL}, 24 \mathrm{mmol})$ and $\mathrm{NaOH}(8.0 \mathrm{~g}, 0.20 \mathrm{~mol})$ in $\mathrm{MeOH} / \mathrm{H}_{2} \mathrm{O}$. Workup A, followed by purification via column chromatography (hexane/ethyl acetate 5:1) provided the title compound as a yellow solid (4.23 g, 82\%). ${ }^{1} \mathrm{H}$ NMR (400 MHz, $\left.\mathrm{CDCl}_{3}\right) \delta 12.79(\mathrm{~s}, 1 \mathrm{H}), 7.92(\mathrm{~d}, 1 \mathrm{H}, J=8.1 \mathrm{~Hz}), 7.88(\mathrm{~d}, 1 \mathrm{H}, J=15.5 \mathrm{~Hz}), 7.64(\mathrm{~d}, 1 \mathrm{H}, J=15.5 \mathrm{~Hz}), 7.42-7.62$ (m, $5 \mathrm{H}), 7.05$ (d, 1H, $J=8.3 \mathrm{~Hz}), 6.97$ (t, 1H, $J=7.7 \mathrm{~Hz}$ ); IR (neat) $v 1644,1565,1486,1441,1406,1369,1263$, 1206, 1091, 1024, 984, 819, 785, 755, $492 \mathrm{~cm}^{-1}$; Anal. Calcd. for $\mathrm{C}_{15} \mathrm{H}_{11} \mathrm{ClO}_{2}: \mathrm{C}, 69.64 ; \mathrm{H}, 4.29$. Found: $\mathrm{C}, 69.71$; 
H, 4.35; mp $145^{\circ} \mathrm{C}\left(\right.$ lit. $\left.^{5} \mathrm{mp} 150{ }^{\circ} \mathrm{C}\right)$.

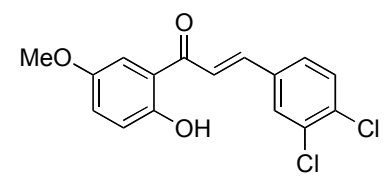

\section{(E)-2'-Hydroxy-5'-methoxy-3,4-dichlorochalcone SM-4}

(E)-2'-Hydroxy-5'-methoxy-3,4-dichlorochalcone was prepared according to the

general procedure using 2'-hydroxy-5'-methoxy-acetophenone (3.3 g, $0.020 \mathrm{~mol}), 3,4$ dichlorobenzaldehyde $(4.2 \mathrm{~g}, 24 \mathrm{mmol})$ and $\mathrm{NaOH}(8.0 \mathrm{~g}, 0.20 \mathrm{~mol})$ in $\mathrm{EtOH} / \mathrm{H}_{2} \mathrm{O}$. Workup A, followed by purification via column chromatography (hexane/ethyl acetate 5:1) provided the title compound as an orange solid (3.84 g, 60\%). ${ }^{1} \mathrm{H}$ NMR (400 MHz, $\left.\mathrm{CDCl}_{3}\right) \delta 12.23(\mathrm{~s}, 1 \mathrm{H}), 7.79(\mathrm{~d}, 1 \mathrm{H}, J=15.5 \mathrm{~Hz}), 7.74(\mathrm{~d}, 1 \mathrm{H}, J=1.9 \mathrm{~Hz})$, 7.46-7.58 (m, 3H), $7.32(\mathrm{~d}, 1 \mathrm{H}, J=3.0 \mathrm{~Hz}), 7.17(\mathrm{dd}, 1 \mathrm{H}, J=3.0,9.1 \mathrm{~Hz}), 6.99(\mathrm{~d}, 1 \mathrm{H}, J=9.1 \mathrm{~Hz}), 3.86(\mathrm{~s}, 3 \mathrm{H})$; ${ }^{13} \mathrm{C} \mathrm{NMR}\left(100 \mathrm{MHz}, \mathrm{CDCl}_{3}\right) \delta 193.0,158.2,152.0,142.9,135.0,134.8,133.6,131.2,130.1,128.0,124.4,121.8$, 119.7, 119.6, 113.0, 56.4; IR (neat) v 2993, 2940, 2836, 1649, 1574, 1490, 1420, 1356, 1269, 1192, 1055, 1016, 853, 822, 765, 726, 704, 683, $537 \mathrm{~cm}^{-1}$; Anal. Calcd. for $\mathrm{C}_{16} \mathrm{H}_{12} \mathrm{Cl}_{2} \mathrm{O}_{3}: \mathrm{C}, 59.46$; H, 3.74. Found: C, 59.24; $\mathrm{H}$, 3.85 ; mp $120-122{ }^{\circ} \mathrm{C}$. A copy of the ${ }^{1} \mathrm{H}$ NMR spectrum is provided.

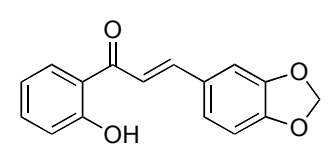

\section{(E)-3-(3,4-Methylendioxyphenyl)-1-(2'hydroxyphenyl)-2-propen-1one SM-5 ${ }^{\mathrm{vi}}$}

(E)-3-(3,4-Methylendioxyphenyl)-1-(2'hydroxyphenyl)-2-propen-1one was prepared according to the general procedure using 2'-hydroxy-acetophenone (1.2 $\mathrm{mL}, 0.010 \mathrm{~mol})$, piperonal $(1.65 \mathrm{~mL}, 11.0 \mathrm{mmol})$ and $\mathrm{NaOH}(4.0 \mathrm{~g}, 0.10 \mathrm{~mol})$ in $\mathrm{EtOH} / \mathrm{H}_{2} \mathrm{O}$. Workup A, followed by recrystallisation from EtOH provided the title compound as a yellow solid (1.88 g, 70\%). ${ }^{1} \mathrm{H} \mathrm{NMR} \mathrm{(400} \mathrm{MHz,}$ $\left.\mathrm{CDCl}_{3}\right) \delta 12.90(\mathrm{~s}, 1 \mathrm{H}), 7.75(\mathrm{dd}, 1 \mathrm{H}, J=1.5,8.0 \mathrm{~Hz}), 7.86(\mathrm{~d}, 1 \mathrm{H}, J=15.3 \mathrm{~Hz}), 7.49-7.53(\mathrm{~m}, 2 \mathrm{H}), 7.16-7.20$ $(\mathrm{m}, 2 \mathrm{H}), 7.03(\mathrm{~d}, 1 \mathrm{H}, J=8.4 \mathrm{~Hz}), 6.95(\mathrm{~m}, 1 \mathrm{H}), 6.87(\mathrm{~d}, 1 \mathrm{H}, J=8.0 \mathrm{~Hz}), 6.06(\mathrm{~s}, 2 \mathrm{H}) ;{ }^{13} \mathrm{C} \mathrm{NMR}(100 \mathrm{MHz}$, $\left.\mathrm{CDCl}_{3}\right) \delta 193,7,163.7,150.5,148.7,145.5,136.4,129.7,129.2,126.0,120.2,119.0,118.8,118.1,108.9,106.9$, 102.0; IR (neat) v 3068, 2909, 1641, 1618, 1569, 1504, 1491, 1373, 1354, 1242, 1203, 1038, 976, $760 \mathrm{~cm}^{-1}$; mp $137{ }^{\circ} \mathrm{C}$ (lit. $\left.{ }^{6 \mathrm{~b}} \mathrm{mp} 124-127^{\circ} \mathrm{C}\right)$. A copy of the ${ }^{1} \mathrm{H}$ NMR spectrum is provided.

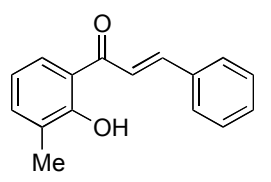

\section{(E)-2'-Hydroxy-3'methyl-chalcone SM-6 ${ }^{\mathrm{vii}}$}

A solution of $o$-cresol $(3.24 \mathrm{~g}, 30.0 \mathrm{mmol})$ in diethyl ether $(30 \mathrm{~mL})$ was added slowly to EtMgBr $\left(3 \mathrm{M}\right.$ in $\left.\mathrm{Et}_{2} \mathrm{O} ; 10.0 \mathrm{~mL}, 0.030 \mathrm{~mol}\right)$ at room temperature under nitrogen atmosphere. The solvent was removed under reduced pressure. The remaining solid was dissolved in toluene $(150 \mathrm{~mL}), \operatorname{tri}(n-$ propyl)amine $(11.0 \mathrm{~mL}, 60.0 \mathrm{mmol})$ and cinnamaldehyde $(7.6 \mathrm{~mL}, 0.060 \mathrm{~mol})$ were added and the reaction mixture was heated under reflux conditions for $5 \mathrm{~h}$. The reaction mixture was cooled to room temperature and an aqueous, saturated solution of $\mathrm{NH}_{4} \mathrm{Cl}(100 \mathrm{~mL})$ was added. The organic phase was separated and the aqueous phase was extracted with diethyl ether $(2 \times 50 \mathrm{~mL})$. The combined organic phases were dried over $\mathrm{MgSO}_{4}$ and the solvent was removed under reduced pressure. The crude material was purified by column chromatography 
(hexane/ethyl acetate 9:1). The title compound was obtained as a yellow solid (1.22 g, 17\%). ${ }^{1} \mathrm{H} \mathrm{NMR}(400 \mathrm{MHz}$, $\left.\mathrm{CDCl}_{3}\right) \delta 13.14(\mathrm{~s}, 1 \mathrm{H}), 7.89(\mathrm{~d}, 1 \mathrm{H}, J=15.5 \mathrm{~Hz}), 7.75(\mathrm{~m}, 1 \mathrm{H}), 7.63-7.67(\mathrm{~m}, 3 \mathrm{H}), 7.40(\mathrm{~m}, 3 \mathrm{H}), 7.35(\mathrm{~m}, 1 \mathrm{H})$, $6.83(\mathrm{t}, 1 \mathrm{H}, J=7.6 \mathrm{~Hz}), 2.28(\mathrm{~s}, 3 \mathrm{H}) ;{ }^{13} \mathrm{C} \mathrm{NMR}\left(100 \mathrm{MHz}, \mathrm{CDCl}_{3}\right) \delta 193.9,162.9,145.2,137.3,134.7,130.9$, 129.1, 128.7, 127.7, 127.4, 120.4, 119.3, 118.3, 15.7; IR (neat) v 3028, 1637, 1597, 1572, 1479, 1449, 1427 , $1342,1302,1265,1241,1223,1078,976,857,753,698,580 \mathrm{~cm}^{-1}$; mp $74{ }^{\circ} \mathrm{C}$ (lit. ${ }^{7} \mathrm{mp} 75-76{ }^{\circ} \mathrm{C}$ ). A copy of the ${ }^{1} \mathrm{H}$ NMR spectrum is provided.

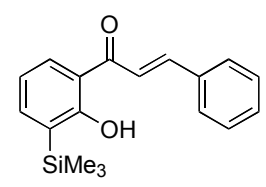

\section{(E)-2'-Hydroxy-3'-trimethylsilylchalcone SM-7}

A solution of $o$-(trimethylsilyl)phenol ${ }^{\text {viii }}(2.33 \mathrm{~g}, 14.0 \mathrm{mmol})$ in diethyl ether $(15 \mathrm{~mL})$ was added slowly to $\operatorname{EtMgBr}\left(3 \mathrm{M}\right.$ in $\left.\mathrm{Et}_{2} \mathrm{O} ; 4.7 \mathrm{~mL}, 14 \mathrm{mmol}\right)$ at room temperature under nitrogen atmosphere. The solvent was removed under reduced pressure. The remaining solid was dissolved in toluene (75 $\mathrm{mL})$, tri( $n$-propyl)amine $(5.3 \mathrm{~mL}, 28 \mathrm{mmol})$ and cinnamaldehyde $(3.53 \mathrm{~mL}, 28.0 \mathrm{mmol})$ were added and the reaction mixture was heated under reflux conditions for $5 \mathrm{~h}$. The reaction mixture was cooled to room temperature and an aqueous, saturated solution of $\mathrm{NH}_{4} \mathrm{Cl}(50 \mathrm{~mL})$ was added. The organic phase was separated and the aqueous phase was extracted with diethyl ether $(2 \times 25 \mathrm{~mL})$. The combined organic phases were dried over $\mathrm{MgSO}_{4}$ and the solvent was removed under reduced pressure. The crude material was purified by column chromatography (hexane/ethyl acetate 9:1). The title compound was obtained as a yellow solid (0.756 g, 18\%). ${ }^{1} \mathrm{H}$ NMR (400 MHz, $\left.\mathrm{CDCl}_{3}\right) \delta 13.16(\mathrm{~s}, 1 \mathrm{H}), 7.93(\mathrm{dd}, 1 \mathrm{H}, J=1.6,8.0 \mathrm{~Hz}), 7.90(\mathrm{~d}, 1 \mathrm{H}, J=15.5 \mathrm{~Hz}), 7.66(\mathrm{~d}, 1 \mathrm{H}, J$ $=15.5 \mathrm{~Hz}), 7.64(\mathrm{~m}, 2 \mathrm{H}), 7.61(\mathrm{dd}, 1 \mathrm{H}, J=1.6,7.1 \mathrm{~Hz}) 7.42(\mathrm{~m}, 3 \mathrm{H}), 6.93(\mathrm{t}, 1 \mathrm{H}, J=7.5 \mathrm{~Hz}), 0.36(\mathrm{~s}, 9 \mathrm{H}) ;{ }^{13} \mathrm{C}$ NMR $\left(100 \mathrm{MHz}, \mathrm{CDCl}_{3}\right) \delta 194.1,168.5,145.2,142.4,134.8,131.0,129.5,129.2,128.8,120.6,118.9,118.7$, -1.0 ; IR (neat) $v 2954,1637,1578,1470,1414,1363,1334,1246,1220,1049,857,841.756 \mathrm{~cm}^{-1} ; \mathrm{mp} 48-50{ }^{\circ} \mathrm{C}$; A copy of the ${ }^{1} \mathrm{H}$ NMR spectrum is provided.

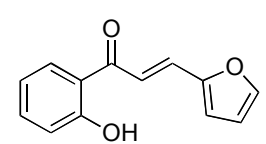

\section{(E)-3-(2-Furanyl)-1-(2'-hydroxyphenyl)-2-propen-1-one SM-8 ${ }^{\text {ix }}$}

(E)-3-(2-Furanyl)-1-(2'-hydroxyphenyl)-2-propen-1-one was prepared according to the general procedure using 2'-hydroxy-acetophenone $(2.4 \mathrm{~mL}, 0.020 \mathrm{~mol})$, 2-furylaldehyde $(1.7 \mathrm{~mL}, 21$ $\mathrm{mmol}$ ) and $\mathrm{NaOH}(8.0 \mathrm{~g}, 0.20 \mathrm{~mol})$ in $\mathrm{EtOH} / \mathrm{H}_{2} \mathrm{O}$. Workup $\mathrm{A}$, followed by purification via column chromatography (hexane/ $\mathrm{CH}_{2} \mathrm{Cl}_{2}$ 1:1) provided the title compound as a yellow solid (2.12 g, 49\%). ${ }^{1} \mathrm{H}$ NMR (400 $\left.\mathrm{MHz}, \mathrm{CDCl}_{3}\right) \delta 12.90(\mathrm{~s}, 1 \mathrm{H}), 7.92(\mathrm{dd}, 1 \mathrm{H}, J=1.5,8.1 \mathrm{~Hz}), 7.69(\mathrm{~d}, 1 \mathrm{H}, J=15.2 \mathrm{~Hz}), 7.48-7.59$ (m, 3H), 7.02 $(\mathrm{d}, 1 \mathrm{H}, J=8.4 \mathrm{~Hz}), 6.95(\mathrm{t}, 1 \mathrm{H}, J=8.1 \mathrm{~Hz}), 6.78(\mathrm{~d}, 1 \mathrm{H}, J=3.4 \mathrm{~Hz}), 6.54(\mathrm{~m}, 1 \mathrm{H})$; IR (neat) $v 3129,1645,1582$, 1554, 1474, 1337, 1271, 1259, 1215, 1160, 1016, 968, $744 \mathrm{~cm}^{-1}$; Anal. Calcd. for $\mathrm{C}_{13} \mathrm{H}_{10} \mathrm{O}_{3}$ : C, 72.89; H, 4.71. Found: $\mathrm{C}, 72.81 ; \mathrm{H}, 4.65 ; \mathrm{mp} 102{ }^{\circ} \mathrm{C}$ (lit. $\left.{ }^{9} \mathrm{mp} 106-108^{\circ} \mathrm{C}\right)$. 


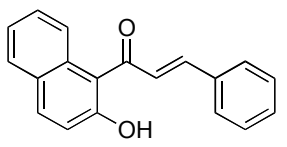

\section{(E)-1-(2'-Hydroxynaphthyl)-3-phenyl-2-propen-1-one SM-9 ${ }^{x}$}

(E)-1-(2'-Hydroxynaphthyl)-3-phenyl-2-propen-1-one was prepared according to the general procedure using 2'-hydroxy-1'-acetonaphthone (2.8 g, $15 \mathrm{mmol})$, benzaldehyde $(1.5 \mathrm{~mL}, 15$ mmol) and $\mathrm{NaOH}(8.0 \mathrm{~g}, 0.20 \mathrm{~mol})$ in $\mathrm{EtOH} / \mathrm{H}_{2} \mathrm{O}$. Workup A, followed by purification via column chromatography (hexane/ $\mathrm{CH}_{2} \mathrm{Cl}_{2} 1: 2$ ) provided the title compound as a yellow solid (2.07 g, 50\%). ${ }^{1} \mathrm{H}$ NMR (400 $\left.\mathrm{MHz}, \mathrm{CDCl}_{3}\right) \delta 12.60(\mathrm{~s}, 1 \mathrm{H}), 8.07(\mathrm{~d}, 1 \mathrm{H}, J=8.5 \mathrm{~Hz}), 7.92-7.96(\mathrm{~m}, 2 \mathrm{H}), 7.83(\mathrm{~d}, 1 \mathrm{H}, J=7.8 \mathrm{~Hz}), 7.40-7.66(\mathrm{~m}$, $7 \mathrm{H}), 7.52(\mathrm{~d}, 1 \mathrm{H}, J=15.7 \mathrm{~Hz}), 7.20(\mathrm{~d}, 1 \mathrm{H}, J=9.0 \mathrm{~Hz}$ ); IR (neat) $v 3061,1635,1597,1571,1463,1407,1370$, 1340, 1243, 1183, 1157, 1107, 995, 825, 759, $716 \mathrm{~cm}^{-1}$; Anal. Calcd. for $\mathrm{C}_{19} \mathrm{H}_{14} \mathrm{O}_{2}: \mathrm{C}, 83.19 ; \mathrm{H}, 5.14$. Found: C, 82.95; H, 5.13; mp $73{ }^{\circ} \mathrm{C}\left(\right.$ lit. $\left.{ }^{10 \mathrm{~b}} \mathrm{mp} 79^{\circ} \mathrm{C}\right)$.

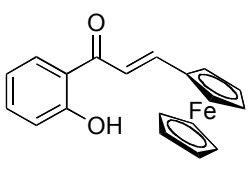

\section{(E)-3-(1-Ferrocenyl)-1-(2'-hydroxyphenyl)-2-propen-1-one SM-10}

(E)-3-(1-Ferrocenyl)-1-(2'-hydroxyphenyl)-2-propen-1-one was prepared according to the general procedure using 2'-hydroxy-acetophenone $(2.4 \mathrm{~mL}, \quad 0.020 \quad \mathrm{~mol})$, ferrocencarboxaldehyde $(4.3 \mathrm{~g}, 0.020 \mathrm{~mol})$ and $\mathrm{NaOH}(8.0 \mathrm{~g}, 0.20 \mathrm{~mol})$ in $\mathrm{EtOH} / \mathrm{H}_{2} \mathrm{O}$. Workup B, followed by purification via column chromatography (hexane/ $\mathrm{CH}_{2} \mathrm{Cl}_{2}$ 1:1) provided the title compound as a purple solid (3.1 g, 47\%). ${ }^{1} \mathrm{H}$ NMR (400 MHz, $\left.\mathrm{CDCl}_{3}\right) \delta 13.11(\mathrm{~s}, 1 \mathrm{H}), 7.91(\mathrm{~d}, 1 \mathrm{H}, J=15.2 \mathrm{~Hz}), 7.87(\mathrm{~m}, 1 \mathrm{H}), 7.48(\mathrm{~m}, 1 \mathrm{H}), 7.26$ (m, 1H), $7.02(\mathrm{~d}, 1 \mathrm{H}, J=8.4 \mathrm{~Hz}), 6.94(\mathrm{t}, 1 \mathrm{H}, J=7.2 \mathrm{~Hz}), 4.65(\mathrm{~m}, 2 \mathrm{H}), 4.56(\mathrm{~m}, 2 \mathrm{H}), 4.22(\mathrm{~s}, 5 \mathrm{H}) ;{ }^{13} \mathrm{C} \mathrm{NMR}$ $\left(100 \mathrm{MHz}, \mathrm{CDCl}_{3}\right) \delta 192.9,163.8,148.2,136.1,129.6,120.2,118.9,118.8,116.8,79.0,72.1,70.1,69.5$; IR (neat) $v 3107,1631,1558,1490,1348,1305,1208,1160,1025,862,831,751,648,498 \mathrm{~cm}^{-1}$; mp $150^{\circ} \mathrm{C}$. A copy of the ${ }^{1} \mathrm{H}$ NMR spectrum is provided.

\section{(E)-2'-Hydroxy- $\alpha$-methylchalcone SM-11 ${ }^{11}$}

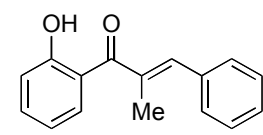

The title compound was synthesized according to the literature procedure. ${ }^{x i}{ }^{1} \mathrm{H}$ NMR (400 $\left.\mathrm{MHz}, \mathrm{CDCl}_{3}\right) \delta 11.85(\mathrm{~s}, 1 \mathrm{H}), 7.75(\mathrm{~m}, 1 \mathrm{H}), 7.47(\mathrm{~m}, 1 \mathrm{H}), 7.31-7.42(\mathrm{~m}, 5 \mathrm{H}), 7.02(\mathrm{~m}, 1 \mathrm{H})$, $6.94(\mathrm{~m}, 1 \mathrm{H}), 6.87(\mathrm{~m}, 1 \mathrm{H}), 2.25(\mathrm{~d}, 3 \mathrm{H}, J=1.5 \mathrm{~Hz}) ;{ }^{13} \mathrm{C} \mathrm{NMR}\left(100 \mathrm{MHz}, \mathrm{CDCl}_{3}\right) \delta 204.2,163.2,138.7,136.2$, 135.8, 135.6, 133.0, 129.7, 128.74, 128.69, 119.1, 118.7, 118.6, 15.6; IR (neat) v 3056, 1622, 1599, 1483, 1446, $1359,1333,1301,1286,1242,1211,1145,1014,925,761,695,524 \mathrm{~cm}^{-1}$; bp $110^{\circ} \mathrm{C}(10 \mathrm{~mm} \mathrm{Hg})\left[\right.$ lit. $^{11}$ bp 140 $\left.{ }^{\circ} \mathrm{C}(1.8 \mathrm{~mm} \mathrm{Hg})\right]$; Anal. Calcd. for $\mathrm{C}_{16} \mathrm{H}_{14} \mathrm{O}_{2}: \mathrm{C}, 80.65 ; \mathrm{H}, 5.92$. Found: $\mathrm{C}, 80.76 ; \mathrm{H}, 6.00$.

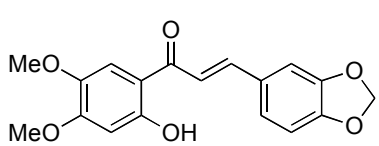

(E)-3-(3,4-methylenedioxyphenyl)-1-(2'-hydroxy-4',5'-dimethoxyphenyl)-2propen-1one SM-12 ${ }^{\mathrm{xii}}$

(E)-3-(3,4-methylenedioxyphenyl)-1-(2'-hydroxy-4',5'-dimethoxyphenyl)-2-propen-1- 
one was prepared according to the general procedure using 2'-hydroxy-4',5'-dimethoxy-acetophenone (3.9 g, $0.020 \mathrm{~mol})$, piperonal $(3.3 \mathrm{~g}, 22 \mathrm{mmol})$ and $\mathrm{NaOH}(8.0 \mathrm{~g}, 0.020 \mathrm{~mol})$ in $\mathrm{EtOH} / \mathrm{H}_{2} \mathrm{O}$. Workup B, followed by purification via column chromatography (hexane/ethyl acetate 1:1) provided the title compound as an orange solid (0.69 g, 11\%). ${ }^{1} \mathrm{H}$ NMR (400 MHz, $\left.\mathrm{CDCl}_{3}\right) \delta 13.43(\mathrm{~s}, 1 \mathrm{H}), 7.81(\mathrm{~d}, 1 \mathrm{H}, J=15.3 \mathrm{~Hz}), 7.32(\mathrm{~d}, 1 \mathrm{H}, J=15.3 \mathrm{~Hz})$, $7.22(\mathrm{~s}, 1 \mathrm{H}), 7.17(\mathrm{~d}, 1 \mathrm{H}, J=1.6 \mathrm{~Hz}), 7.13(\mathrm{dd}, 1 \mathrm{H}, J=1.6,8.0 \mathrm{~Hz}), 6.85$ (d, 1H, $J=8.0 \mathrm{~Hz}), 6.49$ (s, $2 \mathrm{H}), 6.03$ $(\mathrm{s}, 1 \mathrm{H}), 3.92(\mathrm{~s}, 3 \mathrm{H}), 3.91(\mathrm{~s}, 3 \mathrm{H}) ;{ }^{13} \mathrm{C} \mathrm{NMR}\left(100 \mathrm{MHz}, \mathrm{CDCl}_{3}\right) \delta 191.5,161.9,157.1,150.3,148.6,144.6,142.1$, $129.4,125.7,118.4,112.2,110.9,108.9,106.9,101.9,101.0,57.1,56.4$; IR (neat) $v 2930,1637,1575,1514$, 1490, 1447, 1401, 1243, 1194, 1159, 1101, 1039, 989, 804, $588 \mathrm{~cm}^{-1}$; Anal. Calcd. for $\mathrm{C}_{18} \mathrm{H}_{16} \mathrm{O}_{6}: \mathrm{C}, 65.85 ; \mathrm{H}$, 4.91. Found: $\mathrm{C}, 65.67 ; \mathrm{H}, 4.91 ; \mathrm{mp} 180{ }^{\circ} \mathrm{C}$ (lit. $\left.{ }^{12} \mathrm{mp} 182{ }^{\circ} \mathrm{C}\right)$.

\section{General procedure for the synthesis of nonaflates.}

A solution of the corresponding (E)-2'-hydroxy- $\alpha, \beta$-unsaturated ketone (1 equiv), $N, N$-dimethylaminopyridine ( 0.1 equiv) and $i-\mathrm{Pr}_{2} \mathrm{NEt}$ ( 2.4 equiv) in $\mathrm{CH} 2 \mathrm{Cl} 2(2 \mathrm{~mL} / \mathrm{mmol}$ enone) under nitrogen atmosphere was cooled to 0 ${ }^{\circ} \mathrm{C}$ in an ice bath. Perfluoro-1-butanesulfonyl fluoride ( 2 equiv) was added dropwise and the reaction mixture was allowed to warm to room temperature and stirred at that temperature for additional $20 \mathrm{~h}$. Water was added to the reaction mixture $(20 \mathrm{~mL} / \mathrm{mmol}$ enone), the organic layer was separated, washed with brine and dried over $\mathrm{MgSO}_{4}$. The solvent was evaporated under reduced pressure and the crude material was purified by column chromatography on silica gel.

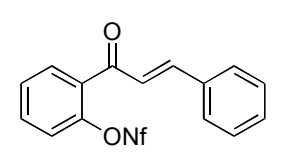

\section{(E)-2-(3-Phenylprop-2-enoyl)phenyl nonafluorobutanesulfonate 1a $(\mathrm{X}=\mathrm{ONf})$}

(E)-2-(3-Phenylprop-2-enoyl)phenyl nonafluorobutanesulfonate was prepared according to the general procedure using $(E)$-2'-hydroxy-chalcone (3.6 g, 16 mmol), DMAP (0.2 g, $1.6 \mathrm{mmol})$, $i$ - $\mathrm{Pr}_{2} \mathrm{NEt}(6.7 \mathrm{~mL}, 38 \mathrm{mmol})$ and $\mathrm{NfF}(5.8 \mathrm{~mL}, 32 \mathrm{mmol})$ in $\mathrm{CH}_{2} \mathrm{Cl}_{2}(30 \mathrm{~mL})$. The crude material was purified by column chromatography (hexane/ethyl acetate 5:1) to give the title compound as a pale yellow solid (7.54 g, 93\%). ${ }^{1} \mathrm{H}$ NMR (400 MHz, $\mathrm{CDCl}_{3}$ ) $\delta 7.77(\mathrm{dd}, 1 \mathrm{H}, J=1.6,7.6 \mathrm{~Hz}), 7.69$ (d, 1H, $\left.J=16.0 \mathrm{~Hz}\right), 7.60(\mathrm{~m}, 3 \mathrm{H}), 7.50$ $(\mathrm{t}, 1 \mathrm{H}, J=7.5 \mathrm{~Hz}), 7.41(\mathrm{~m}, 4 \mathrm{H}), 7.22(\mathrm{~d}, 1 \mathrm{H}, J=16.0 \mathrm{~Hz}) ;{ }^{13} \mathrm{C} \mathrm{NMR}\left(100 \mathrm{MHz}, \mathrm{CDCl}_{3}\right) \delta 190.4,147.6,147.6$, $134.8,133.8,133.6,131.7,131.3,129.6,129.3,129.1,125.2,123.1 ;{ }^{19} \mathrm{~F} \mathrm{NMR}\left(376 \mathrm{MHz}, \mathrm{CDCl}_{3}\right) \delta-85.2$, -113.6, -125.2, -130.3; IR (neat) v3065, 3030, 1673, 1651, 1610, 1427, 1354, 1202, 1144, 1033, 904, 884, 770, 748, 696, 586, 568, $521 \mathrm{~cm}^{-1}$; Anal. Calcd. for $\mathrm{C}_{19} \mathrm{H}_{11} \mathrm{~F}_{9} \mathrm{O}_{4} \mathrm{~S}: \mathrm{C}, 45.07$; H, 2.19. Found: C, 45.19; H, 2.11; mp 45$47^{\circ} \mathrm{C}$. A copy of the ${ }^{1} \mathrm{H}$ NMR spectrum is provided.

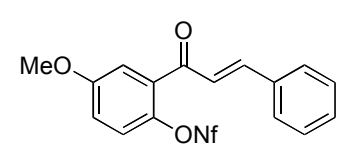

(E)-4-Methoxy-2-(3-phenylprop-2-enoyl)phenyl nonafluorobutanesulfonate 1b

(E)-4-Methoxy-2-(3-phenylprop-2-enoyl)phenyl nonafluorobutanesulfonate was prepared 
according to the general procedure using (E)-2'-hydroxy-5'methoxy-chalcone SM-1 (3.22 g, 12.7 mmol), DMAP (0.16 g, $1.3 \mathrm{mmol}), i$ - $\mathrm{Pr}_{2} \mathrm{NEt}(5.3 \mathrm{~mL}, 30 \mathrm{mmol})$ and $\mathrm{NfF}(4.5 \mathrm{~mL}, 25 \mathrm{mmol})$ in $\mathrm{CH}_{2} \mathrm{Cl}_{2}(25 \mathrm{~mL})$. The crude material was purified by column chromatography $\left(\mathrm{CH}_{2} \mathrm{Cl}_{2}\right.$ /hexane 2:1) to give the title compound as a yellow solid (3.88 g, 57\%). ${ }^{1} \mathrm{H}$ NMR (400 MHz, $\left.\mathrm{CDCl}_{3}\right) \delta 7.70(\mathrm{~d}, 1 \mathrm{H}, J=16.0 \mathrm{~Hz}), 7.60-7.62(\mathrm{~m}, 2 \mathrm{H}), 7.41-7.43(\mathrm{~m}$, $3 \mathrm{H}), 7.32(\mathrm{~d}, 1 \mathrm{H}, J=9.0 \mathrm{~Hz}), 7.22(\mathrm{~d}, 1 \mathrm{H}, J=3.1 \mathrm{~Hz}), 7.19(\mathrm{~d}, 1 \mathrm{H}, J=16.0 \mathrm{~Hz}), 7.08(\mathrm{dd}, 1 \mathrm{H}, J=3.0,9.0 \mathrm{~Hz})$, $3.88(\mathrm{~s}, 3 \mathrm{H}) ;{ }^{13} \mathrm{C}$ NMR $\left(100 \mathrm{MHz}, \mathrm{CDCl}_{3}\right) \delta 189.7,159.1,147.1,140.4,134.40,134.37,131.2,129.1,128.8$, 124.7, 123.8, 117.9, 115.47, 56.0; ${ }^{19} \mathrm{~F}$ NMR (376 MHz, $\left.\mathrm{CDCl}_{3}\right) \delta-85.0,-113.5,-125.1,-130.2$; IR (neat) $v$ 3065, 3029, 2944, 2843, 1673, 1603, 1489, 1424, 1353, 1333, 1201, 1144, 1034, 884, 770, 739, 698, $588 \mathrm{~cm}^{-1}$; Anal. Calcd. for $\mathrm{C}_{20} \mathrm{H}_{13} \mathrm{~F}_{9} \mathrm{O}_{5} \mathrm{~S}$ : C, 44.79; H, 2.44. Found: C, 44.83; H, 2.33; mp 74-76 ${ }^{\circ} \mathrm{C}$. A copy of the ${ }^{1} \mathrm{H}$ NMR spectrum is provided.

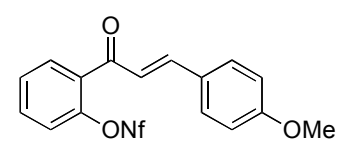

\section{(E)-2-[3-(4-Methoxyphenyl)prop-2-enoyl]phenyl nonafluorobutanesulfonate 1c}

(E)-2-[3-(4-Methoxyphenyl)prop-2-enoyl]phenyl nonafluorobutanesulfonate was prepared $27 \mathrm{mmol})$, DMAP (0.33 g, $2.7 \mathrm{mmol}), i-\operatorname{Pr}_{2} \mathrm{NEt}(11.3 \mathrm{~mL}, 64.8 \mathrm{mmol})$ and NfF (9.7 mL, $\left.54 \mathrm{mmol}\right)$ in CH2Cl2 (50 $\mathrm{mL}$ ). The crude material was purified by column chromatography (hexane/ethyl acetate 4:1) to give the title compound as a pale yellow solid (5.28 g, 37\%). ${ }^{1} \mathrm{H}$ NMR (400 MHz, $\left.\mathrm{CDCl}_{3}\right) \delta 7.75(\mathrm{dd}, 1 \mathrm{H}, J=1.6,7.6 \mathrm{~Hz})$, $7.64(\mathrm{~d}, 1 \mathrm{H}, J=15.9 \mathrm{~Hz}), 7.49-7.61(\mathrm{~m}, 4 \mathrm{H}), 7.41(\mathrm{~d}, 1 \mathrm{H}, J=8.2 \mathrm{~Hz}), 7.08(\mathrm{~d}, 1 \mathrm{H}, J=15.9 \mathrm{~Hz}), 6.93(\mathrm{~d}, 2 \mathrm{H}, 8.7$ $\mathrm{Hz}), 3.86(\mathrm{~s}, 3 \mathrm{H}) ;{ }^{13} \mathrm{C} \mathrm{NMR}\left(100 \mathrm{MHz}, \mathrm{CDCl}_{3}\right) \delta 190.0,162.3,147.2,133.7,132.9,130.8,128.6,127.1,122.6$, 114.7, 55.6; ${ }^{19} \mathrm{~F}$ NMR $\left(376 \mathrm{MHz}, \mathrm{CDCl}_{3}\right) \delta-85.0,-113.5,-125.1,-130.2$; IR (neat) $v 3083,3009,2947,2918$, 2847, 1662, 1588, 1512, 1420, 1354, 1333, 1296, 1202, 1026, 984, 914, 891, 835, 807, 776, $524 \mathrm{~cm}^{-1}$; Anal. Calcd. for $\mathrm{C}_{20} \mathrm{H}_{13} \mathrm{~F}_{9} \mathrm{O}_{5} \mathrm{~S}$ : C, 44.79; H, 2.44. Found: $\mathrm{C}, 44.76 ; \mathrm{H}, 2.33 ; \mathrm{mp} 60^{\circ} \mathrm{C}$. A copy of the ${ }^{1} \mathrm{H}$ NMR spectrum is provided.

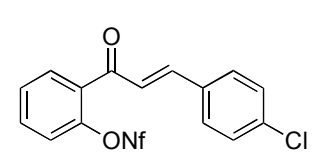

\section{(E)-2-[3-(4-Chloroyphenyl)prop-2-enoyl]phenyl nonafluorobutanesulfonate 1d}

(E)-2-[3-(4-Chloroyphenyl)prop-2-enoyl]phenyl nonafluorobutanesulfonate was prepared according to the general procedure using $(E)-2$ '-hydroxy-4-chlorochalcone SM-3 (2.6 g, 10 mmol), DMAP (0.122 g, $1.00 \mathrm{mmol}), i$-Pr 2 NEt (4.2 mL, $24 \mathrm{mmol})$ and NfF (3.6 mL, $20 \mathrm{mmol})$ in CH2Cl2 (35 $\mathrm{mL}$ ). The crude material was purified by column chromatography (hexane/ethyl acetate 5:1) to give the title compound as a pale yellow solid $(4.0 \mathrm{~g}, 74 \%) .{ }^{1} \mathrm{H} \mathrm{NMR}\left(400 \mathrm{MHz}, \mathrm{CDCl}_{3}\right) \delta 7.77$ (dd, $\left.1 \mathrm{H}, J=1.8,7.6 \mathrm{~Hz}\right)$, 7.62-7.66 (m, 2H), 7.51-7.55 (m, 3H), 7.39-7.43 (m, 3H), $7.17(\mathrm{~d}, 1 \mathrm{H}, J=16.0 \mathrm{~Hz}),{ }^{13} \mathrm{C}$ NMR $\left(100 \mathrm{MHz}, \mathrm{CDCl}_{3}\right)$ $\delta 189.7,147.2,145.5,137.3,133.3,132.9,130.9,130.0,129.6,128.7,125.2,122.8 ;{ }^{19} \mathrm{~F}$ NMR $\left(376 \mathrm{MHz}, \mathrm{CDCl}_{3}\right)$ $\delta-85.0,-113.4,-125.1,-130.2$; IR (neat) $v 3086,1669,1610,1492,1418,1333,1203,1143,1110,1022,1010$, 
909, 826, 774, $500 \mathrm{~cm}^{-1}$; Anal. Calcd. for $\mathrm{C}_{19} \mathrm{H}_{10} \mathrm{ClF}_{9} \mathrm{O}_{4} \mathrm{~S}$ : C, 42.20; H, 1.86. Found: C, 42.34; H, 1.60; mp 97-99 ${ }^{\circ} \mathrm{C}$. A copy of the ${ }^{1} \mathrm{H}$ NMR spectrum is provided.

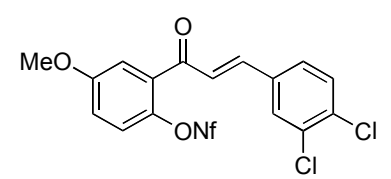

(E)-2-[3-(3,4-Dichlorophenyl)prop-2-enoyl]-4-methoxyphenyl nonafluorobutanesulfonate $1 \mathrm{e}$

(E)-2-[3-(3,4-Dichlorophenyl)prop-2-enoyl]-4-methoxyphenyl nonafluorobutane sulfonate was prepared according to the general procedure using (E)-2'-hydroxy-5'-methoxy-3,4dichlorochalcone SM-4 (1.6 g, $5.0 \mathrm{mmol})$, DMAP (0.30 g, $2.5 \mathrm{mmol}), i$-Pr 2 NEt (2.0 mL, $12 \mathrm{mmol})$ and NfF (1.8 $\mathrm{mL}, 0.010 \mathrm{~mol})$ in $\mathrm{CH} 2 \mathrm{Cl} 2(50 \mathrm{~mL})$. The crude material was purified by column chromatography (hexane/ethyl acetate $6: 1)$ to give the title compound as a yellow solid $(3.00 \mathrm{~g}, 99 \%) .{ }^{1} \mathrm{H} \mathrm{NMR}\left(400 \mathrm{MHz}, \mathrm{CDCl}_{3}\right) \delta 7.64(\mathrm{~d}, 1 \mathrm{H}$, $J=1.7 \mathrm{~Hz}), 7.57(\mathrm{~d}, 1 \mathrm{H}, J=16.0 \mathrm{~Hz}), 7.40-7.46(\mathrm{~m}, 2 \mathrm{H}), 7.29(\mathrm{~d}, 1 \mathrm{H}, J=9.2 \mathrm{~Hz}), 7.22(\mathrm{~d}, 1 \mathrm{H}, J=3.2 \mathrm{~Hz}), 7.17$ $(\mathrm{d}, 1 \mathrm{H}, J=16.0 \mathrm{~Hz}), 7.08(\mathrm{dd}, 1 \mathrm{H}, J=3.2,9.2 \mathrm{~Hz}), 3.86(\mathrm{~s}, 3 \mathrm{H}) ;{ }^{13} \mathrm{C} \mathrm{NMR}\left(100 \mathrm{MHz}, \mathrm{CDCl}_{3}\right) \delta 189.0,159.1$, $143.8,140.4,135.0,134.4,133.9,133.4,131.1,130.3,130.2,127.7,126.0,123.8,118.2,115.5,56.0 ;{ }^{19} \mathrm{~F} \mathrm{NMR}$ $\left(376 \mathrm{MHz}, \mathrm{CDCl}_{3}\right) \delta-85.0,-113.5,-125.2,-130.2$; IR (neat) $v 3089,3015,2944,2842,1675,1607,1553,1473$, 1424, 1199, 1033, 983, 858, 823, 740, 698, 589, $532 \mathrm{~cm}^{-1}$; Anal. Calcd. for $\mathrm{C}_{20} \mathrm{H}_{11} \mathrm{~F}_{9} \mathrm{O}_{5} \mathrm{~S}: \mathrm{C}, 39.69 ; \mathrm{H}, 1.83$. Found: $\mathrm{C}, 39.79 ; \mathrm{H}, 1.75 ; \mathrm{mp} 55^{\circ} \mathrm{C}$. A copy of the ${ }^{1} \mathrm{H}$ NMR spectrum is provided.

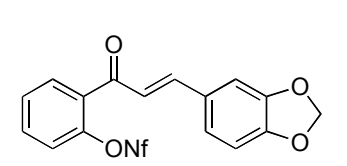

2-[(2E)-3-(1,3-Benzodioxol-5-yl)-2-propenoyl]phenyl nonafluorobutanesulfonate if 2-[(2E)-3-(1,3-Benzodioxol-5-yl)-2-propenoyl]phenyl nonafluorobutanesulfonate was prepared according to the general procedure using (E)-3-(3,4-methylenedioxyphenyl)-1-(2'hydroxyphenyl)-2-propen-1one SM-5 (1.34 g, $5.00 \mathrm{mmol})$, DMAP (0.06 g, $0.5 \mathrm{mmol}), i$-Pr $\mathrm{NEt}_{2}(2.1 \mathrm{~mL}, 12$ $\mathrm{mmol})$ and $\mathrm{NfF}(1.8 \mathrm{~mL}, 0.010 \mathrm{~mol})$ in $\mathrm{CH} 2 \mathrm{Cl} 2(20 \mathrm{~mL})$. The crude material was purified by column chromatography (hexane/ethyl acetate 5:1) to give the title compound as a pale yellow solid (1.86 g, 68\%). ${ }^{1} \mathrm{H}$ NMR (400 MHz, $\left.\mathrm{CDCl}_{3}\right) \delta 7.75(\mathrm{dd}, 1 \mathrm{H}, J=1.8,7.6 \mathrm{~Hz}), 7.58-7.64(\mathrm{~m}, 2 \mathrm{H}), 7.49-7.53(\mathrm{t}, 1 \mathrm{H}, J=7.6 \mathrm{~Hz}), 7.40$ $(\mathrm{d}, 1 \mathrm{H}, J=8.2 \mathrm{~Hz}), 7.08-7.12(\mathrm{~m}, 2 \mathrm{H}), 7.03(\mathrm{~d}, 1 \mathrm{H}, J=15.8 \mathrm{~Hz}), 6.84(\mathrm{~d}, 1 \mathrm{H}, J=8.0 \mathrm{~Hz}), 6.04(\mathrm{~s}, 2 \mathrm{H}) ;{ }^{13} \mathrm{C} \mathrm{NMR}$ $\left(100 \mathrm{MHz}, \mathrm{CDCl}_{3}\right) \delta 189.8,150.6,148.7,147.2,147.0,133.6,133.0,130.8,128.9,128.6,126.0,122.9,122.6$, 108.9, 106.9, 101.9; ${ }^{19} \mathrm{~F}$ NMR $\left(376 \mathrm{MHz}, \mathrm{CDCl}_{3}\right) \delta-81.3,-109.9,-121.5,-126.6$; IR (neat) v 3084, 2920, 1636, 1601, 1504, 1452, 1420, 1297, 1266, 1232, 1203, 1148, 1059, 1039, 988, 923, 906, 812, 774, 761, 752, 694, 590, $517 \mathrm{~cm}^{-1}$; Anal. Calcd. for $\mathrm{C}_{20} \mathrm{H}_{11} \mathrm{~F}_{9} \mathrm{O}_{6} \mathrm{~S}$ : C, 43.65; H, 2.01. Found: C, 43.85; H, 1.98; mp $94{ }^{\circ} \mathrm{C}$. A copy of the ${ }^{1} \mathrm{H}$ NMR spectrum is provided.

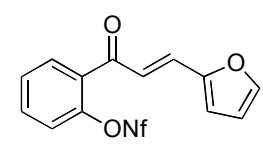

(E)-2-[3-(Furan-2-yl)prop-2-enoyl]phenyl nonafluorobutanesulfonate 1i

(E)-2-[3-(Furan-2-yl)prop-2-enoyl]phenyl nonafluorobutanesulfonate was prepared according 
to the general procedure using (E)-3-(2-Furanyl)-1-(2'-hydroxyphenyl)-2-propen-1-one SM-8 (2.12 g, 9.87 mmol), DMAP (0.12 g, $0.99 \mathrm{mmol}), i$-Pr $2 \mathrm{NEt}(4.2 \mathrm{~mL}, 24 \mathrm{mmol})$ and $\mathrm{NfF}(3.6 \mathrm{~mL}, 0.020 \mathrm{~mol})$ in $\mathrm{CH}_{2} \mathrm{Cl}_{2}(35$ $\mathrm{mL}$ ). The crude material was purified by column chromatography (hexane/ethyl acetate 5:1) to give the title compound as a yellow solid (3.83 g, 80\%). ${ }^{1} \mathrm{H}$ NMR (400 MHz, $\left.\mathrm{CDCl}_{3}\right) \delta 7.78(\mathrm{dd}, 1 \mathrm{H}, J=1.8,7.6 \mathrm{~Hz}), 7.39$ $7.60(\mathrm{~m}, 5 \mathrm{H}), 7.11(\mathrm{~d}, 1 \mathrm{H}, J=15.5 \mathrm{~Hz}), 6.76(\mathrm{~d}, 1 \mathrm{H} . J=3.4 \mathrm{~Hz}), 6.53(\mathrm{dd}, 1 \mathrm{H}, J=1.8 \mathrm{~Hz}, 3.4 \mathrm{~Hz}) ;{ }^{13} \mathrm{C} \mathrm{NMR}$ $\left(100 \mathrm{MHz}, \mathrm{CDCl}_{3}\right) \delta 189.3,151.3,147.2,145.9,133.5,133.1,132.5,130.8,128.7,122.8,122.0,117.6,113.1 ;{ }^{19} \mathrm{~F}$ NMR (376 MHz, $\left.\mathrm{CDCl}_{3}\right) \delta-85.0,-113.6,-125.2,-130.3$; IR (neat) $v 3126,3035,1670,1603,1551,1475,1426$, 1355, 1290, 1200, 1140, 1015, 899, 777, 761, 694, 572, $521 \mathrm{~cm}^{-1}$; Anal. Calcd. for $\mathrm{C}_{17} \mathrm{H}_{9} \mathrm{~F}_{9} \mathrm{O}_{5} \mathrm{~S}: \mathrm{C}, 41.14 ; \mathrm{H}, 1.83$. Found: $\mathrm{C}, 41.25 ; \mathrm{H}, 1.74 ; \mathrm{mp} 50-52^{\circ} \mathrm{C}$. A copy of the ${ }^{1} \mathrm{H}$ NMR spectrum is provided.

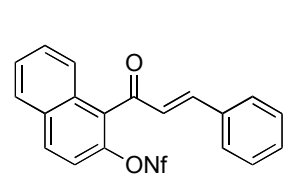

\section{(E)-1-(3-phenylprop-2-enoyl)naphthalen-2-yl nonafluorobutanesulfonate $1 \mathrm{k}$}

(E)-1-(3-phenylprop-2-enoyl)naphthalen-2-yl nonafluorobutanesulfonate was prepared according to the general procedure using (E)-1-(2'-hydroxynaphthyl)-3-phenyl-2-propen-1one SM-9 (2.07 g, $7.54 \mathrm{mmol})$, DMAP (90.0 g, $0.754 \mathrm{mmol}), i$-Pr $2 \mathrm{NEt}(3.2 \mathrm{~mL}, 18 \mathrm{mmol})$ and NfF $(2.7 \mathrm{~mL}, 15$ mmol) in $\mathrm{CH}_{2} \mathrm{Cl}_{2}(15 \mathrm{~mL})$. The crude material was purified by column chromatography (hexane $\left./ \mathrm{CH}_{2} \mathrm{Cl}_{2} 1: 1\right)$ to give the title compound as a pale yellow solid (3.65 g, 87\%). ${ }^{1} \mathrm{H}$ NMR (400 MHz, $\left.\mathrm{CDCl}_{3}\right) \delta 8.05(\mathrm{~d}, 1 \mathrm{H}, J=9.1$ $\mathrm{Hz}), 7.98(\mathrm{~m}, 1 \mathrm{H}), 7.87(\mathrm{~m}, 1 \mathrm{H}), 7.51-7.63(\mathrm{~m}, 5 \mathrm{H}), 7.38-7.42(\mathrm{~m}, 4 \mathrm{H}), 7.18(\mathrm{~d}, 1 \mathrm{H}, J=16.2 \mathrm{~Hz}) ;{ }^{13} \mathrm{C} \mathrm{NMR}$ $\left(100 \mathrm{MHz}, \mathrm{CDCl}_{3}\right) \delta 193.2,148.9,143.3,134.1,132.6,132.1,131.5,131.2,130.4,129.2,129.0,128.7,128.6$, 127.9, 127.7, 125.8, 119.3; ${ }^{19} \mathrm{~F}$ NMR $\left(376 \mathrm{MHz}, \mathrm{CDCl}_{3}\right) \delta-85.0,-113.6,-125.1,-130.2$; IR (neat) v 3063, 1653, 1623, 1598, 1577, 1509, 1427, 1240, 1204, 1145, 1102, 1033, 1005, 947, 814, 793, 751, $689 \mathrm{~cm}^{-1}$; Anal. Calcd. for $\mathrm{C}_{23} \mathrm{H}_{13} \mathrm{~F}_{9} \mathrm{O}_{4} \mathrm{~S}$ : C, 49.65; H, 2.36. Found: C, 49.79; H, 2.33; mp 83-85 ${ }^{\circ} \mathrm{C}$. A copy of the ${ }^{1} \mathrm{H}$ NMR spectrum is provided.

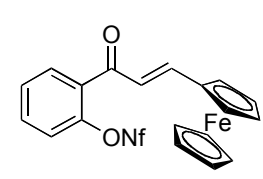

\section{(E)-2-[3-(1-Ferrocenyl)prop-2-enoyl]phenyl nonafluorobutanesulfonate 11}

(E)-2-[3-(1-Ferrocenyl)prop-2-enoyl]phenyl nonafluorobutanesulfonate was prepared according to the general procedure using (E)-3-(1-ferrocenyl)-1-(2'-hydroxyphenyl)-2-propen-1-one SM10 (458 mg, $1.38 \mathrm{mmol})$, DMAP (17 mg, $0.14 \mathrm{mmol}), i-\mathrm{Pr}_{2} \mathrm{NEt}(0.60 \mathrm{~mL}, 3.5 \mathrm{mmol})$ and NfF $(0.50 \mathrm{~mL}, 2.8$ $\mathrm{mmol})$ in $\mathrm{CH}_{2} \mathrm{Cl}_{2}(20 \mathrm{~mL})$. The crude material was purified by column chromatography (hexane/ethyl acetate 5:1) to give the title compound as a red solid (321 mg, 38\%). ${ }^{1} \mathrm{H}$ NMR (400 MHz, $\left.\mathrm{CDCl}_{3}\right) \delta 7.72(\mathrm{dd}, 1 \mathrm{H}, J=1.8,7.6$ $\mathrm{Hz}), 7.64(\mathrm{~d}, 1 \mathrm{H}, J=15.6 \mathrm{~Hz}), 7.59-7.63(\mathrm{~m}, 1 \mathrm{H}), 7.51(\mathrm{t}, 1 \mathrm{H}, J=7.5 \mathrm{~Hz}), 7.41(\mathrm{~d}, 1 \mathrm{H}, J=8.2 \mathrm{~Hz}), 6.79(\mathrm{~d}, 1 \mathrm{H}, J$ $=15.6 \mathrm{~Hz}), 4.58(\mathrm{~m}, 2 \mathrm{H}), 4.53(\mathrm{~m}, 2 \mathrm{H}), 4.21(\mathrm{~s}, 5 \mathrm{H}) ;{ }^{13} \mathrm{C} \mathrm{NMR}\left(100 \mathrm{MHz}, \mathrm{CDCl}_{3}\right) \delta 189.3,149.9,147.1,134.1$, 132.6, 130.8, 128.5, 122.5, 122.2, 78.5, 72.1, 70.2, 70.1, 69.6; ${ }^{19} \mathrm{~F}$ NMR (376 $\left.\mathrm{MHz}, \mathrm{CDCl}_{3}\right) \delta-84.9,-113.4$, $-125.1,-130.1$; IR (neat) $v 3097,2924,1664,1641,1608,1584,1427,1240,1202,1144,1023,899,776 \mathrm{~cm}^{-1}$; 
$\mathrm{mp} 80^{\circ} \mathrm{C}$. A copy of the ${ }^{1} \mathrm{H}$ NMR spectrum is provided.

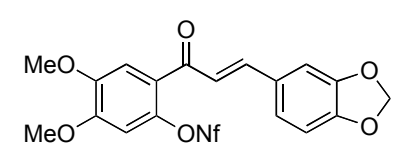

trifluoromethanesulfonate

\section{(E)-2-(3-(3,4-methylendioxy)prop-2-enoyl)-4,5-dimethoxyphenyl}

\section{trifluoromethanesulfonate $1 \mathrm{n}$}

(E)-2-(3-(3,4-methylendioxy)prop-2-enoyl)-4,5-dimethoxyphenyl methylenedioxyphenyl)-1-(2'-hydroxy-4',5'-dimethoxyphenyl)-2-propen-1-one SM-12 (0.550 g, 1.68 mmol), DMAP $(0.21 \mathrm{~g}, 0.17 \mathrm{mmol}), i-\mathrm{Pr}_{2} \mathrm{NEt}(0.7 \mathrm{~mL}, 4 \mathrm{mmol})$ and $\mathrm{NfF}(0.60 \mathrm{~mL}, 3.4 \mathrm{mmol})$ in $\mathrm{CH}_{2} \mathrm{Cl}_{2}(20 \mathrm{~mL})$. The crude material was purified by column chromatography (hexane/ethyl acetate 1:1) to give the title compound as a yellow solid (0.81 g, 80\%). ${ }^{1} \mathrm{H}$ NMR (400 MHz, $\left.\mathrm{CDCl}_{3}\right) \delta 7.65(\mathrm{~d}, 1 \mathrm{H}, J=15.6 \mathrm{~Hz}), 7.23(\mathrm{~s}, 1 \mathrm{H}), 7.10(\mathrm{~d}, 1 \mathrm{H}, J=$ $1.7 \mathrm{~Hz}), 7.08(\mathrm{dd}, 1 \mathrm{H}, J=8.2 \mathrm{~Hz}, J=1.7 \mathrm{~Hz}), 7.06(\mathrm{~d}, 1 \mathrm{H}, J=15.6 \mathrm{~Hz}), 6.81(\mathrm{~d}, 1 \mathrm{H}, J=8.0 \mathrm{~Hz}), 6.79(\mathrm{~s}, 1 \mathrm{H})$, $6.01(\mathrm{~s}, 2 \mathrm{H}), 3.94(\mathrm{~s}, 3 \mathrm{H}), 3.93(\mathrm{~s}, 3 \mathrm{H}) ;{ }^{13} \mathrm{C} \mathrm{NMR}\left(100 \mathrm{MHz}, \mathrm{CDCl}_{3}\right) \delta 188.3,152.4,150.5,148.6,145.9,141.3$, $129.1,125.9,125.7,122.8,112.1,108.9,106.9,105.6,101.9,56.7,56.6 ;{ }^{19} \mathrm{~F} \mathrm{NMR}\left(376 \mathrm{MHz}, \mathrm{CDCl}_{3}\right) \delta-80.8$, $-109.2,-121.0,-126.0$; IR (neat) v 2910, 1663, 1608, 1588, 1518, 1491, 1449, 1426, 1354, 1245, 12012, 1144, 1102, 1036, 980, 932, 891, 851, 807, 738, 585, $531 \mathrm{~cm}^{-1}$; Anal. Calcd. for $\mathrm{C}_{22} \mathrm{H}_{15} \mathrm{~F}_{9} \mathrm{O}_{8} \mathrm{~S}: \mathrm{C}, 43.29 ; \mathrm{H}, 2.48$. Found: $\mathrm{C}, 43.42 ; \mathrm{H}, 2.48 ; \mathrm{mp} 105^{\circ} \mathrm{C}$. A copy of the ${ }^{1} \mathrm{H}$ NMR spectrum is provided.

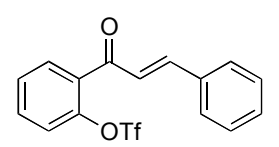

\section{(E)-2-(3-Phenylprop-2-enoyl)phenyl trifluoromethanesulfonate 1a $(\mathrm{X}=$ OTf $)$}

A solution of $(E)-2$ '-hydroxy-chalcone $(2.92 \mathrm{~g}, 13.0 \mathrm{mmol})$ in pyridine $(15 \mathrm{~mL})$ was cooled to $0{ }^{\circ} \mathrm{C}$ in an ice bath. Trifluoromethanesulfonic anhydride $(2.9 \mathrm{~mL}, 17 \mathrm{mmol})$ was added dropwise and the reaction mixture was stirred additional $10 \mathrm{~min}$ at that temperature before warming the solution to room temperature. After $12 \mathrm{~h}$ the solution was diluted with $\mathrm{Et}_{2} \mathrm{O}(150 \mathrm{~mL})$, washed with an aqueous, saturated solution of $\mathrm{CuSO}_{4}(10 \times 50 \mathrm{~mL})$ and water $(50 \mathrm{~mL})$. The organic phase was dried over $\mathrm{MgSO}_{4}$ and the solvent was evaporated under reduced pressure. The crude material was purified by column chromatography on silica gel (hexane/ethyl acetate 4:1) to yield the desired product as a colorless oil (3.88 g, 84\%). ${ }^{1} \mathrm{H}$ NMR (400 MHz, $\left.\mathrm{CDCl}_{3}\right) \delta 7.78(\mathrm{dd}, 1 \mathrm{H}, J=1.4,7.5 \mathrm{~Hz}), 7.68(\mathrm{~d}, 1 \mathrm{H}, J=16 \mathrm{~Hz}), 7.60-7.66(\mathrm{~m}, 3 \mathrm{H}), 7.53(\mathrm{t}, 1 \mathrm{H}, J=7.5 \mathrm{~Hz}), 7.40-$ $7.45(\mathrm{~m}, 4 \mathrm{H}), 7.21(\mathrm{~d}, 1 \mathrm{H}, J=16.0 \mathrm{~Hz}) ;{ }^{13} \mathrm{C} \mathrm{NMR}\left(100 \mathrm{MHz}, \mathrm{CDCl}_{3}\right) \delta 189.8,147.0,134.3,133.2,131.2,130.8$, 129.2, 128.8, 128.6, 124.8, 122.6, 120.3, 117.1; $\left.{ }^{19} \mathrm{~F} \mathrm{NMR} \mathrm{(376} \mathrm{MHz,} \mathrm{CDCl}_{3}\right) \delta-77.6$; IR (neat) $v 3064,1673$, 1650, 1610, 1576, 1483, 1425, 1333, 1302, 1210, 1139, 1021, 981, 903, 881, 768, 697, 596, 570, $519 \mathrm{~cm}^{-1}$; Anal. Calcd. for $\mathrm{C}_{16} \mathrm{H}_{11} \mathrm{~F}_{3} \mathrm{O}_{4} \mathrm{~S}$ : C, 53.93; H, 3.11. Found: C, 54.22; H, 3.10. A copy of the ${ }^{1} \mathrm{H}$ NMR spectrum is provided. 


\section{(E)-2-methyl-6-(3-phenylprop-2-enoyl)phenyl trifluoromethanesulfonate $1 \mathrm{~g}$}

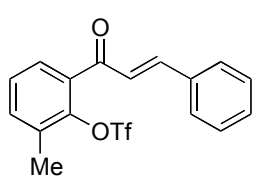

A solution of $(E)-2$ '-hydroxy-3'methyl-chalcone SM-6 $(1.43 \mathrm{~g}, 6.00 \mathrm{mmol})$ in pyridine (10 $\mathrm{mL}$ ) was cooled to $0{ }^{\circ} \mathrm{C}$ in an ice bath. Trifluoromethanesulfonic anhydride $(1.3 \mathrm{~mL}, 7.8 \mathrm{mmol})$ was added dropwise and the reaction mixture was stirred additional $10 \mathrm{~min}$ at that temperature before warming the solution to room temperature. After $12 \mathrm{~h}$ the solution was diluted with $\mathrm{Et}_{2} \mathrm{O}(100 \mathrm{~mL})$, washed with an aqueous, saturated solution of $\mathrm{CuSO}_{4}(10 \times 50 \mathrm{~mL})$ and water $(50 \mathrm{~mL})$. The organic phase was dried over $\mathrm{MgSO}_{4}$ and the solvent was evaporated under reduced pressure. The crude material was purified by column chromatography on silica gel (hexane/ethyl acetate 5:1) to yield the desired product as a white solid (1.4 g, 63\%). ${ }^{1} \mathrm{H}$ NMR (400 MHz, $\left.\mathrm{CDCl}_{3}\right) \delta 7.61(\mathrm{~d}, 1 \mathrm{H}, J=16.0 \mathrm{~Hz}), 7.55-7.58(\mathrm{~m}, 2 \mathrm{H}), 7.50(\mathrm{~m}, 1 \mathrm{H}), 7.44(\mathrm{~m}, 1 \mathrm{H})$, 7.34-7.40 (m, 4H), $7.10(\mathrm{~d}, 1 \mathrm{H}, J=16.0 \mathrm{~Hz}), 2.45(\mathrm{~s}, 3 \mathrm{H}) ;{ }^{13} \mathrm{C}$ NMR $\left(100 \mathrm{MHz}, \mathrm{CDCl}_{3}\right) \delta 191.0,146.9,144.8$, 135.1, 134.5, 134.4, 133.0, 131.2, 129.2, 128.8, 128.5, 128.3, 125.3, 17.1; $\left.{ }^{19} \mathrm{~F} \mathrm{NMR} \mathrm{(376} \mathrm{MHz,} \mathrm{CDCl}_{3}\right) \delta-73.8$; IR (neat) $v 3063,1674,1650,1604,1577,1450,1423,1333,1300,1212,1140,1077,980,888,766,613 \mathrm{~cm}^{-1}$; Anal. Calcd. for $\mathrm{C}_{17} \mathrm{H}_{13} \mathrm{~F}_{3} \mathrm{O}_{4} \mathrm{~S}$ : C, 55.13; H, 3.54. Found: C, 55.11; H, 3.51; mp $55{ }^{\circ} \mathrm{C}$. A copy of the ${ }^{1} \mathrm{H}$ NMR spectrum is provided.

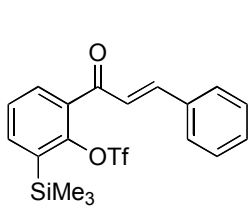

\section{(E)-2-(3-phenylprop-2-enoyl)-6-(trimethylsilyl)phenyl trifluoromethanesulfonate $1 \mathrm{~h}$}

$n$-BuLi (2.5M in hexane; $1.1 \mathrm{~mL}, 2.8 \mathrm{mmol})$ was added to a solution on di(i-propyl)amine $(0.4$ $\mathrm{mL}, 2.8 \mathrm{mmol})$ in THF $(15 \mathrm{~mL})$ at $-10{ }^{\circ} \mathrm{C}$. The solution was stirred at $-10{ }^{\circ} \mathrm{C}$ for $30 \mathrm{~min}$, cooled to $-78{ }^{\circ} \mathrm{C}$ and transferred via cannula to a solution of (E)-2'-hydroxy-3'trimethylsilylchalcone SM-7 (663 mg, $2.24 \mathrm{mmol})$ in THF $(15 \mathrm{~mL})$ at $-78{ }^{\circ} \mathrm{C}$. After $40 \mathrm{~min} \mathrm{Tf}_{2} \mathrm{O}(0.77 \mathrm{~mL}, 4.6$ mmol) was slowly added and the reaction mixture was allowed to warm to $0{ }^{\circ} \mathrm{C}$ over a time period of $3 \mathrm{~h}$. An aqueous, saturated solution of $\mathrm{NH}_{4} \mathrm{Cl}(20 \mathrm{~mL})$ was added at $0{ }^{\circ} \mathrm{C}$, the organic phase was separated and washed with an aqueous, saturated solution of $\mathrm{NaCl}(20 \mathrm{~mL})$. The organic phase was dried over $\mathrm{MgSO}_{4}$ and the solvent was evaporated under reduced pressure. The crude material was purified by column chromatography on silica gel (hexane/ethyl acetate 7:1) to yield the desired product as a yellow solid (504 g, 52\%). ${ }^{1} \mathrm{H}$ NMR (400 MHz, $\left.\mathrm{CD}_{2} \mathrm{Cl}_{2}\right) \delta 7.78(\mathrm{dd}, 1 \mathrm{H}, J=1.9,7.4 \mathrm{~Hz}), 7.70(\mathrm{dd}, 2 \mathrm{H}, J=1.9,7.5 \mathrm{~Hz}), 7.59-7.63(\mathrm{~m}, 3 \mathrm{H}), 7.53(\mathrm{t}, 1 \mathrm{H}, J=7.5$ $\mathrm{Hz}), 7.43(\mathrm{~m}, 3 \mathrm{H}), 7.12(\mathrm{~d}, 1 \mathrm{H}, J=16.1 \mathrm{~Hz}), 0.45(\mathrm{~s}, 9 \mathrm{H}) ;{ }^{13} \mathrm{C} \mathrm{NMR}\left(100 \mathrm{MHz}, \mathrm{CD}_{2} \mathrm{Cl}_{2}\right) \delta 191.1,149.0,146.6$, $139.9,137.0,134.9,132.3,131.5,129.6,129.1,128.6,125.9,123.6,120.4,117.2,0.15 ;{ }^{19} \mathrm{~F}$ NMR $(376 \mathrm{MHz}$, $\left.\mathrm{CD}_{2} \mathrm{Cl}_{2}\right) \delta-73.7$; IR (neat) $v 2960,1673,1603,1575,1449,1401,1391,1334,1211,1137,1117,1056,1042$, $844,775,741.650 \mathrm{~cm}^{-1} ; \mathrm{mp} 109-111^{\circ} \mathrm{C}$. A copy of the ${ }^{1} \mathrm{H}$ NMR spectrum is provided.

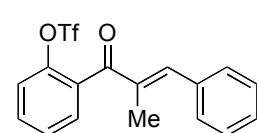

(E)-2-(2-methyl-3-phenylprop-2-enoyl)phenyl trifluoromethanesulfonate 1m

A solution of (E)-2'-hydroxy- $\alpha$-methyl-chalcone SM-11 (1.92 g, $8.06 \mathrm{mmol})$ in pyridine (12 
$\mathrm{mL}$ ) was cooled to $0{ }^{\circ} \mathrm{C}$ in an ice bath. Trifluoromethanesulfonic anhydride $(1.80 \mathrm{~mL}, 10.5 \mathrm{mmol})$ was added dropwise and the reaction mixture was stirred additional $10 \mathrm{~min}$ at that temperature before warming the solution to room temperature. After $12 \mathrm{~h}$ the solution was diluted with $\mathrm{Et}_{2} \mathrm{O}(100 \mathrm{~mL})$, washed with an aqueous, saturated solution of $\mathrm{CuSO}_{4}(10 \times 50 \mathrm{~mL})$ and water $(50 \mathrm{~mL})$. The organic phase was dried over $\mathrm{MgSO}_{4}$ and the solvent was evaporated under reduced pressure. The crude material was purified by column chromatography on silica gel (hexane/ethyl acetate $7: 1)$ to yield the desired product as a colorless oil $(1.53 \mathrm{~g}, 51 \%) .{ }^{1} \mathrm{H}$ NMR $(400 \mathrm{MHz}$, $\left.\mathrm{CDCl}_{3}\right) \delta 7.52-7.58(\mathrm{~m}, 2 \mathrm{H}), 7.45(\mathrm{~m}, 1 \mathrm{H}), 7.31-7.38(\mathrm{~m}, 6 \mathrm{H}), 7.13(\mathrm{~m}, 1 \mathrm{H}), 2.22(\mathrm{~m}, 3 \mathrm{H}) ;{ }^{13} \mathrm{C} \mathrm{NMR}(100 \mathrm{MHz}$ $\left.\mathrm{CDCl}_{3}\right) \delta 195.3,146.4,145.8,137.6,135.4,133.6,132.1,130.9,130.1,129.4,128.7,128.4,122.3,13.3 ;{ }^{19} \mathrm{~F}$ NMR $\left(376 \mathrm{MHz}, \mathrm{CDCl}_{3}\right) \delta-73.9$; IR (neat) v 3065, 1656, 1621, 1424, 1248, 1214, 1139, 1090, 1015, 928, 884, 770, $694,591,518 \mathrm{~cm}^{-1}$; A copy of the ${ }^{1} \mathrm{H}$ NMR spectrum is provided.

\section{General procedure (I) for the reductive-Heck reaction with proton sponge.}

An oven-dried screw-cap test tube equipped with a Teflon septum was charged with a magnetic stirbar, the corresponding nonaflate or triflate (1 equiv), $\mathrm{Pd}(\mathrm{OAc})_{2}(5 \mathrm{~mol} \%),(R)-3,5$-XylMeOBIPHEP $(10 \mathrm{~mol} \%)$ and proton sponge ( 2 equiv). The tube was evacuated and backfilled with argon; this procedure was carried out two times. The solids were dissolved in DMF ( $4 \mathrm{~mL} / \mathrm{mmol}$ nonaflate or triflate), the reaction tube was sealed and the reaction mixture was stirred in a pre-heated oil-bath at $100{ }^{\circ} \mathrm{C}$ for $12 \mathrm{~h}$. The reaction mixture was cooled to room temperature, diluted with ethyl acetate $(20 \mathrm{~mL} / \mathrm{mmol}$ nonaflate) and washed with an aqueous solution of $\mathrm{HCl}$ $\left(1 \mathrm{M}, 20 \mathrm{~mL} / \mathrm{mmol}\right.$ nonaflate). The organic phase was separated, washed with brine and dried over $\mathrm{MgSO}_{4}$. The solvent was evaporated under reduced pressure and the crude material was purified by column chromatography on silica gel.

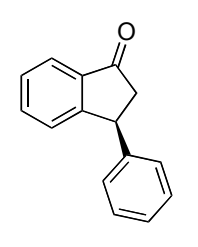

\section{(3S)-Phenylindan-1-one (2a) $)^{\text {xii }}$}

Compound 2a was prepared according to the general procedure (I) using (E)-2-(3-phenylprop-2enoyl)phenyl nonafluorobutanesulfonate 1a $(\mathrm{X}=\mathrm{ONf})(152 \mathrm{mg}, 0.300 \mathrm{mmol}), \mathrm{Pd}(\mathrm{OAc})_{2}(3.4 \mathrm{mg}$, $0.015 \mathrm{mmol}),(R)-3,5$-XylMeOBIPHEP $(21 \mathrm{mg}, 0.030 \mathrm{mmol})$ and proton sponge $(129 \mathrm{mg}, 0.600$ mmol) in DMF (1.2 mL). The crude material was purified by column chromatography (hexane/ethyl acetate 5:1) to give the title compound as a white solid $\left(56 \mathrm{mg}, 90 \%, 78 \%\right.$ ee). ${ }^{1} \mathrm{H}$ NMR $\left(400 \mathrm{MHz}, \mathrm{CDCl}_{3}\right) \delta 7.82(\mathrm{~d}, 1 \mathrm{H}, J=$ $7.7 \mathrm{~Hz}), 7.58(\mathrm{t}, 1 \mathrm{H}, J=7.5 \mathrm{~Hz}), 7.43(\mathrm{t}, 1 \mathrm{H}, J=7.5 \mathrm{~Hz}), 7.24-7.35(\mathrm{~m}, 4 \mathrm{H}), 7.14(\mathrm{~m}, 2 \mathrm{H}), 4.57$, (dd, 1H, $J=3.8$, $8.0 \mathrm{~Hz}), 3.24(\mathrm{dd}, 1 \mathrm{H}, J=8.0,19.2 \mathrm{~Hz}), 2.73(\mathrm{dd}, 1 \mathrm{H}, J=3.9,19.2 \mathrm{~Hz}) ;{ }^{13} \mathrm{C} \mathrm{NMR}\left(100 \mathrm{MHz}, \mathrm{CDCl}_{3}\right) \delta 206.6$, $158.5,144.2,137.3,135.7,129.5,128.4,128.2,127.5,127.5,123.9,47.4,45.0 ; \mathrm{mp} 35^{\circ} \mathrm{C}$; the enantiomeric excess of 2a was determined by HPLC analysis (Chiracel OJ column, $i-\mathrm{PrOH} /$ hexane 10:90; $1.0 \mathrm{~mL} / \mathrm{min}, 254$ $\mathrm{nm}) ;(3 R)$ isomer (minor) $t_{\mathrm{R}}=8.8 \mathrm{~min}$ and $(3 S)$ isomer (major) $t_{\mathrm{R}}=16.5 \mathrm{~min} ;[\alpha]_{\mathrm{D}}=+48.2\left(\mathrm{c}=0.76, \mathrm{CHCl}_{3}\right)$, 
$\left[\right.$ lit. $^{13 \mathrm{a}}[\alpha]_{\mathrm{D}}=+61.2(\mathrm{c}=2.5, \mathrm{EtOH})$ for a sample of $\left.90 \% e e\right]$. A copy of the NMR spectra is provided.

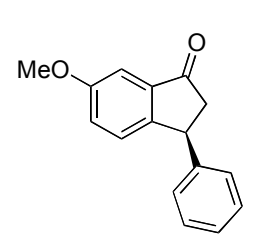

\section{6-Methoxy-(3S)-phenylindan-1-one $(2 \mathrm{~b})^{\mathrm{xiv}}$}

Compound 2b was prepared according to the general procedure (I) using (E)-4-methoxy-2-(3phenylprop-2-enoyl)phenyl nonafluorobutanesulfonate $\mathbf{1 b}(268 \mathrm{mg}, 0.500 \mathrm{mmol}), \mathrm{Pd}(\mathrm{OAc})_{2}$ (5.6 mg, $0.025 \mathrm{mmol}),(R)-3,5-X y l M e O B I P H E P ~(35 \mathrm{mg}, 0.050 \mathrm{mmol}$ ) and proton sponge (214 $\mathrm{mg}, 1.00 \mathrm{mmol})$ in DMF $(2 \mathrm{~mL})$. The crude material was purified by column chromatography (hexane/ethyl acetate 5:1) to give the title compound as a white solid (104 mg, 87\%, 76\% ee). ${ }^{1} \mathrm{H}$ NMR (400 MHz, $\left.\mathrm{CDCl}_{3}\right) \delta$ 7.24-7.34 (m, 4H), $7.17(2 \mathrm{H}, J=1.5 \mathrm{~Hz}), 7.13(\mathrm{~m}, 2 \mathrm{H}), 4.53(\mathrm{dd}, 1 \mathrm{H}, J=3.6,7.8 \mathrm{~Hz}), 3.87(\mathrm{~s}, 3 \mathrm{H}), 3.27(\mathrm{dd}, 1 \mathrm{H}$, $J=7.8,19.2 \mathrm{~Hz}), 2.71(\mathrm{dd}, 1 \mathrm{H}, J=3.6 \mathrm{~Hz}, 19.2 \mathrm{~Hz}) ;{ }^{13} \mathrm{C} \mathrm{NMR}\left(100 \mathrm{MHz}, \mathrm{CDCl}_{3}\right) \delta 206.0,159.7,150.8,143.9$, 138.0, 128.8, 127.6, 127.5, 126.9, 124.5, 104.3, 55.6, 47.5, 43.7; IR (neat) v 3400, 3027, 2940, 2836, 1709, 1613, 1489, 1331, 1282, 1243, 1045, 1026, 839, 760, $701 \mathrm{~cm}^{-1}$; Anal. Calcd. for $\mathrm{C}_{16} \mathrm{H}_{14} \mathrm{O}_{2}: \mathrm{C}, 80.65 ; \mathrm{H}, 5.92$. Found: C, 80.36; H, 5.84; mp $74-75{ }^{\circ} \mathrm{C}$; the enantiomeric excess of $\mathbf{2 b}$ was determined by HPLC analysis (Chiracel OJ column, $i-\mathrm{PrOH} /$ hexane $5: 95 ; 1.0 \mathrm{~mL} / \mathrm{min}, 254 \mathrm{~nm}$ ); $(3 R)$ isomer (minor) $t_{\mathrm{R}}=18.1 \mathrm{~min}$ and $(3 S)$ isomer (major) $t_{\mathrm{R}}$ $=22.3 \mathrm{~min} ;[\alpha]_{\mathrm{D}}=+43.3\left(\mathrm{c}=0.6, \mathrm{CHCl}_{3}\right)$.

\section{(3S)-(4-Methoxyphenyl)indan-1-one (2c) ${ }^{\mathrm{xV}}$}

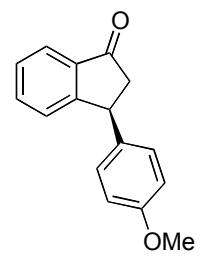

Compound 2c was prepared according to the general procedure (I) using (E)-2-[3-(4methoxyphenyl)prop-2-enoyl]phenyl nonafluorobutanesulfonate 1c (268 $\mathrm{mg}, 0.500 \mathrm{mmol})$, $\mathrm{Pd}(\mathrm{OAc})_{2}(5.6 \mathrm{mg}, 0.025 \mathrm{mmol}),(R)-3,5-\mathrm{XylMeOBIPHEP}(35 \mathrm{mg}, 0.050 \mathrm{mmol})$ and proton sponge $(214 \mathrm{mg}, 1.00 \mathrm{mmol})$ in DMF $(2 \mathrm{~mL})$. The crude material was purified by column chromatography (hexane/ethyl acetate 5:1) to give the title compound as a yellow solid (106 $\mathrm{mg}, 89 \%, 70 \%$ ee). ${ }^{1} \mathrm{H}$ NMR (400 MHz, $\left.\mathrm{CDCl}_{3}\right) \delta 7.82(\mathrm{~d}, 1 \mathrm{H}, J=7.7 \mathrm{~Hz}), 7.57$ (t, $\left.1 \mathrm{H}, J=7.6 \mathrm{~Hz}\right), 7.42(\mathrm{t}, 1 \mathrm{H}, J=7.4 \mathrm{~Hz}), 7.27$ (d, $1 \mathrm{H}, J=7.8 \mathrm{~Hz}), 7.05(\mathrm{~m}, 2 \mathrm{H}), 6.86(\mathrm{~m}, 2 \mathrm{H}), 4.54(\mathrm{dd}, 1 \mathrm{H}, J=3.8,7.9 \mathrm{~Hz}), 3.80(\mathrm{~s}, 3 \mathrm{H}), 3.22(\mathrm{dd}, 1 \mathrm{H}, J=8.0$, $19.2 \mathrm{~Hz}), 2.66(\mathrm{dd}, 1 \mathrm{H}, J=3.8,19.2 \mathrm{~Hz}) ;{ }^{13} \mathrm{C} \mathrm{NMR}\left(100 \mathrm{MHz}, \mathrm{CDCl}_{3}\right) \delta 206.3,158.6,158.4,136.8,135.8,135.2$, 128.7, 127.9, 126.9, 123.4, 114.3, 55.4, 47.1, 43.8; IR (neat) v 3033, 2948, 2833, 1698, 1602, 1511, 1462, 1266, 1248, 1178, 1033, 826, $761 \mathrm{~cm}^{-1}$; Anal. Calcd. for $\mathrm{C}_{16} \mathrm{H}_{14} \mathrm{O}_{2}$ : C, 80.65; H, 5.92. Found: C, 80.42; H, 5.92; mp 75$77^{\circ} \mathrm{C}$; the enantiomeric excess of $\mathbf{2 c}$ was determined by HPLC analysis (Chiracel OJ column, $i$-PrOH/hexane $5: 95 ; 1.0 \mathrm{~mL} / \mathrm{min}, 254 \mathrm{~nm}$ ); $(3 R)$ isomer (minor) $t_{\mathrm{R}}=22.8 \mathrm{~min}$ and $(3 S)$ isomer (major) $t_{\mathrm{R}}=37.9 \mathrm{~min} ;[\alpha]_{\mathrm{D}}=$ $+41.1\left(\mathrm{c}=0.6, \mathrm{CHCl}_{3}\right)$.

(3S)-(4-Chlorophenyl)indan-1-one (2d) ${ }^{\mathrm{xvi}}$

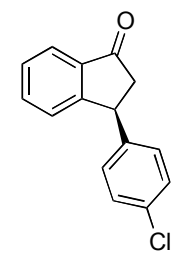


Compound 2d was prepared according to the general procedure (I) using (E)-2-[3-(4-chloroyphenyl)prop-2enoyl]phenyl nonafluorobutanesulfonate $1 \mathbf{d}(270 \mathrm{mg}, 0.500 \mathrm{mmol}), \mathrm{Pd}(\mathrm{OAc})_{2}(5.6 \mathrm{mg}, 0.025 \mathrm{mmol}),(R)-3,5-$ XylMeOBIPHEP (35 mg, $0.050 \mathrm{mmol})$ and proton sponge $(214 \mathrm{mg}, 1.00 \mathrm{mmol})$ in DMF (2 mL). The crude material was purified by column chromatography (hexane/ethyl acetate 5:1) to give the title compound as a pale yellow solid (98 mg, 81\%, 77\% ee). ${ }^{1} \mathrm{H}$ NMR (400 MHz, $\left.\mathrm{CDCl}_{3}\right) \delta 7.81$ (d, 1H, $\left.J=7.7 \mathrm{~Hz}\right), 7.59$ (t, $1 \mathrm{H}, J=7.3$ $\mathrm{Hz}), 7.43$ (t, 1H, $J=7.6 \mathrm{~Hz}), 7.24-7.29(\mathrm{~m}, 3 \mathrm{H}), 7.06(\mathrm{~m}, 2 \mathrm{H}), 4.56(\mathrm{dd}, 1 \mathrm{H}, J=3.8,8.0 \mathrm{~Hz}), 3.23(\mathrm{dd}, 1 \mathrm{H}, J=$ 8.0, 19.2 Hz), $2.63(\mathrm{dd}, 1 \mathrm{H}, J=3.8,19.2 \mathrm{~Hz}) ;{ }^{13} \mathrm{C} \mathrm{NMR}\left(100 \mathrm{MHz}, \mathrm{CDCl}_{3}\right) \delta 205.5,157.3,142.2,136.7,135.2$, 132.7, 129.0, 129.0, 128.1, 126.7, 123.5, 46.7, 43.8; IR (neat) v 3408, 3030, 2919, 1712, 1603, 1491, 1462, 1091, 1044, 1014, 827, $761 \mathrm{~cm}^{-1}$; Anal. Calcd. for $\mathrm{C}_{15} \mathrm{H}_{11} \mathrm{ClO}$ : C, 74.23; H, 4.57. Found: C, 73.95; H, 4.54; mp $50{ }^{\circ} \mathrm{C}$; the enantiomeric excess of $\mathbf{2 d}$ was determined by HPLC analysis (Chiracel OJ column, $i$-PrOH/hexane 5:95; 1.0 $\mathrm{mL} / \mathrm{min}, 254 \mathrm{~nm}) ;(3 R)$ isomer (minor) $t_{\mathrm{R}}=15.6 \mathrm{~min}$ and $(3 S)$ isomer (major) $t_{\mathrm{R}}=17.6 \mathrm{~min} ;[\alpha]_{\mathrm{D}}=+42.9(\mathrm{c}=$ $\left.0.6, \mathrm{CHCl}_{3}\right)$.

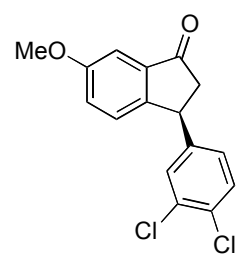

\section{(3S)-(3,4-Dichlorophenyl)-6-methoxyindan-1-one $(2 \mathrm{e})^{\mathrm{xvii}}$}

Compound 2e was prepared according to the general procedure (I) using (E)-2-[3-(3,4Dichlorophenyl)prop-2-enoyl]-4-methoxyphenyl nonafluorobutanesulfonate 1e (310 mg, 0.512 $\mathrm{mmol}), \mathrm{Pd}(\mathrm{OAc})_{2}(5.8 \mathrm{mg}, 0.026 \mathrm{mmol}),(R)-3,5-X y l M e O B I P H E P(35.6 \mathrm{mg}, 0.0512 \mathrm{mmol})$ and proton sponge $(220 \mathrm{mg}, 1.02 \mathrm{mmol})$ in DMF $(2 \mathrm{~mL})$. The crude material was purified by column chromatography (hexane/ethyl acetate 5:1) to give the title compound as a colorless viscous oil (127 $\mathrm{mg}, 81 \%$, $74 \%$ ee). ${ }^{1} \mathrm{H}$ NMR (400 MHz, $\left.\mathrm{CDCl}_{3}\right) \delta 7.35$ (d, $\left.1 \mathrm{H}, J=8.3 \mathrm{~Hz}\right), 7.12-7.22(\mathrm{~m}, 4 \mathrm{H}), 6.93$ (dd, $1 \mathrm{H}, J=1.8,8.2$ $\mathrm{Hz}), 4.48(\mathrm{dd}, 1 \mathrm{H}, J=3.4,7.7 \mathrm{~Hz}), 3.85(\mathrm{~s}, 3 \mathrm{H}), 3.23(\mathrm{dd}, 1 \mathrm{H}, J=7.9,19.2 \mathrm{~Hz}), 2.61(\mathrm{dd}, 1 \mathrm{H}, J=3.4,19.2 \mathrm{~Hz})$; ${ }^{13} \mathrm{C}$ NMR $\left(100 \mathrm{MHz}, \mathrm{CDCl}_{3}\right) \delta 204.9,160.1,149.4,144.4,138.2,132.9,131.1,130.9,129.6,127.6,127.0,124.8$, 104.8, 55.8, 47.3, 43.0; IR (neat) v 3402, 3062, 2940, 2836, 1710, 1614, 1490, 1470, 1432, 1395, 1333, 1283, $1243,1176,1131,1045,1029,839,824,708,558 \mathrm{~cm}^{-1}$; the enantiomeric excess of $\mathbf{2 e}$ was determined by HPLC analysis (Chiracel OD-H column, $i$-PrOH/hexane 3:97; $1.0 \mathrm{~mL} / \mathrm{min}, 254 \mathrm{~nm}$ ); (3S) isomer (major) $t_{\mathrm{R}}=14.7 \mathrm{~min}$ and $(3 R)$ isomer (minor) $t_{\mathrm{R}}=17.0 \mathrm{~min} ;[\alpha]_{\mathrm{D}}=+44.1\left(\mathrm{c}=1.23, \mathrm{CHCl}_{3}\right)$. A copy of the NMR spectra is provided.

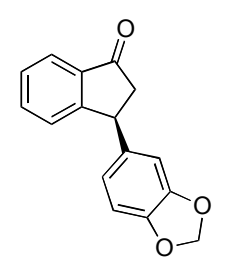

\section{(3S)-(3,4-Methylendioxyphenyl)-indan-1-one (2f) $)^{13 \mathrm{~b}}$}

Compound $2 \mathbf{f}$ was prepared according to the general procedure (I) using 2-[(2E)-3-(1,3benzodioxol-5-yl)-2-propenoyl]phenyl nonafluorobutanesulfonate 1 f $(275 \mathrm{mg}, 0.500 \mathrm{mmol})$, $\mathrm{Pd}(\mathrm{OAc})_{2}(5.6 \mathrm{mg}, 0.025 \mathrm{mmol}),(R)-3,5$-XylMeOBIPHEP $(35 \mathrm{mg}, 0.050 \mathrm{mmol})$ and proton sponge $(214 \mathrm{mg}, 1.00 \mathrm{mmol})$ in DMF $(2 \mathrm{~mL})$. The crude material was purified by column chromatography (hexane/ethyl acetate $7: 1$ ) to give the title compound as a light yellow solid (102 mg, 81\%, 69\% ee). ${ }^{1} \mathrm{H}$ NMR 
$\left(400 \mathrm{MHz}, \mathrm{CDCl}_{3}\right) \delta 7.80(\mathrm{~d}, 1 \mathrm{H}, J=7.7 \mathrm{~Hz}), 7.57(\mathrm{t}, 1 \mathrm{H}, J=7.6 \mathrm{~Hz}), 7.42(\mathrm{t}, 1 \mathrm{H}, J=7.5 \mathrm{~Hz}), 7.29(\mathrm{~d}, 1 \mathrm{H}, J=$ $7.7 \mathrm{~Hz}), 6.75(\mathrm{~d}, 1 \mathrm{H}, J=7.9 \mathrm{~Hz}), 6.65(\mathrm{~d}, 1 \mathrm{H}, J=7.9 \mathrm{~Hz}), 6.51(\mathrm{~m}, 1 \mathrm{H}), 5.93(\mathrm{~m}, 2 \mathrm{H}), 4.51(\mathrm{dd}, 1 \mathrm{H}, J=3.8,8.0$ $\mathrm{Hz}), 3.20(\mathrm{dd}, 1 \mathrm{H}, J=8.0,19.2 \mathrm{~Hz}), 2.63(\mathrm{dd}, 1 \mathrm{H}, J=3.8,19.2 \mathrm{~Hz}) ;{ }^{13} \mathrm{C} \mathrm{NMR}\left(100 \mathrm{MHz}, \mathrm{CDCl}_{3}\right) \delta 206.0,158.0$, 148.3, 146.7, 137.7, 136.8, 135.2, 128.0, 126.9, 123.5, 121.0, 108.5, 107.9, 101.2, 47.1, 44.3; IR (neat) v 3404, $2898,1711,1602,1502,1487,1443,1246,1038,934,813,761 \mathrm{~cm}^{-1}$; $\mathrm{mp} 95-97{ }^{\circ} \mathrm{C}$; the enantiomeric excess of $2 \mathbf{f}$ was determined by HPLC analysis (Chiracel OJ column, $i$-PrOH/hexane 5:95; $1.0 \mathrm{~mL} / \mathrm{min}, 254 \mathrm{~nm}$ ); (3R) isomer (minor) $t_{\mathrm{R}}=26.2 \mathrm{~min}$ and $(3 S)$ isomer (major) $t_{\mathrm{R}}=33.6 \mathrm{~min} ;[\alpha]_{\mathrm{D}}=+31.3\left(\mathrm{c}=1, \mathrm{CHCl}_{3}\right.$ ). ), $\left[\right.$ lit. $^{13 \mathrm{~b}}[\alpha]_{\mathrm{D}}=+28.6$ $\left(\mathrm{c}=1.85, \mathrm{CHCl}_{3}\right)$ for a sample of $\left.63 \% e e\right]$. A copy of the NMR spectra is provided.

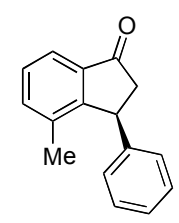

\section{4-Methyl-(3S)-phenylindan-1-one (2g)}

Compound 2g was prepared according to the general procedure (I) using (E)-2-methyl-6-(3phenylprop-2-enoyl)phenyl trifluoromethanesulfonate $1 \mathrm{~g}$ (185 mg, $0.500 \mathrm{mmol}), \mathrm{Pd}(\mathrm{OAc})_{2}(5.6 \mathrm{mg}$, $0.025 \mathrm{mmol}),(R)-3,5-\mathrm{Xyl}-\mathrm{MeO}-\mathrm{BIPHEP}(35 \mathrm{mg}, 0.050 \mathrm{mmol})$ and proton sponge $(214 \mathrm{mg}, 1.00$ $\mathrm{mmol}$ ) in DMF (2 mL). The crude material was purified by column chromatography (hexane/ethyl acetate 5:1) to give the title compound as a yellow solid $\left(100 \mathrm{mg}, 90 \%, 94 \%\right.$ ee). ${ }^{1} \mathrm{H}$ NMR $\left(400 \mathrm{MHz}, \mathrm{CDCl}_{3}\right) \delta 7.65(\mathrm{~m}, 1 \mathrm{H})$, 7.31-7.36 (m, 2H), 7.15-7.25 (m, 3H), $7.00(\mathrm{~m}, 2 \mathrm{H}), 4.55(\mathrm{dd}, 1 \mathrm{H}, J=2.5,8.3 \mathrm{~Hz}), 3.20$ (dd, $1 \mathrm{H}, J=8.3,19.3$ $\mathrm{Hz}), 2.56(\mathrm{dd}, 1 \mathrm{H}, J=2.6,19.3 \mathrm{~Hz}), 1.98(\mathrm{~s}, 3 \mathrm{H}) ;{ }^{13} \mathrm{C} \mathrm{NMR}\left(100 \mathrm{MHz}, \mathrm{CDCl}_{3}\right) \delta 206.7,155.7,143.7,137.3$, 136.9, 136.6, 129.0, 128.5, 127.4, 126.8, 121.1, 47.7, 43.9, 18.5; IR (neat) v 3026, 2920, 1713, 1604, 1587, 1494, $1481,1454,1320,1280,1054,781,762,702 \mathrm{~cm}^{-1}$; mp $35^{\circ} \mathrm{C}$; the enantiomeric excess of $\mathbf{2 g}$ was determined by HPLC analysis (Chiracel OJ column, $i-\mathrm{PrOH} /$ hexane 5:95; $1.0 \mathrm{~mL} / \mathrm{min}, 254 \mathrm{~nm}$ ); (3S) isomer (major) $t_{\mathrm{R}}=11.0$ min and $(3 R)$ isomer (minor) $t_{\mathrm{R}}=13.2 \mathrm{~min} ;[\alpha]_{\mathrm{D}}=-47.7\left(\mathrm{c}=0.95, \mathrm{CHCl}_{3}\right)$; A copy of the NMR spectra is provided.

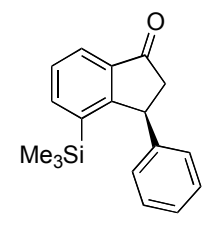

\section{4-Trimethylsilyl-(3S)-phenylindan-1-one (2h)}

Compound $\mathbf{2 h}$ was prepared according to the general procedure (I) using (E)-2-(3-phenylprop-2enoyl)-6-(trimethylsilyl)phenyl trifluoromethanesulfonate $\mathbf{1 h}$ (129 $\mathrm{mg}, 0.300 \mathrm{mmol}), \mathrm{Pd}(\mathrm{OAc})_{2}$ (3.4 mg, $0.015 \mathrm{mmol}),(R)-3,5$-XylMeOBIPHEP (21 mg, $0.03 \mathrm{mmol}$ ) and proton sponge (129 $\mathrm{mg}$, $0.600 \mathrm{mmol}$ ) in DMF $(1.2 \mathrm{~mL})$. The crude material was purified by column chromatography (hexane/ethyl acetate $7: 1)$ to give the title compound as a yellow solid (49.6 mg, 59\%, 86\% ee). ${ }^{1} \mathrm{H}$ NMR $\left(400 \mathrm{MHz}, \mathrm{CD}_{2} \mathrm{Cl}_{2}\right) \delta 7.83$ $(\mathrm{m}, 2 \mathrm{H}), 7.47(\mathrm{~m}, 1 \mathrm{H}), 7.18-7.27(\mathrm{~m}, 3 \mathrm{H}), 6.90(\mathrm{~m}, 2 \mathrm{H}), 4.82(\mathrm{dd}, 1 \mathrm{H}, J=1.9,8.4 \mathrm{~Hz}), 3.20(\mathrm{dd}, 1 \mathrm{H}, J=8.4,19.0$ $\mathrm{Hz}), 2.45(\mathrm{dd}, 1 \mathrm{H}, J=2.0,19.0 \mathrm{~Hz}), 0.01(\mathrm{~s}, 9 \mathrm{H}) ;{ }^{13} \mathrm{C} \mathrm{NMR}\left(100 \mathrm{MHz}, \mathrm{CD}_{2} \mathrm{Cl}_{2}\right) \delta 206.7,162.7,146.4,142.4$, $139.9,137.4,129.3,128.4,128.0,127.3,124.3,48.3,45.8,-0.3$; IR (neat) $v 3061,3028,2954,1713,1587,1569$, $1495,14698,1454,1407,1279,1249,873,839,784,702 \mathrm{~cm}^{-1}$; mp $35^{\circ} \mathrm{C}$; the enantiomeric excess of $\mathbf{2 h}$ was 
determined by HPLC analysis (Chiracel OD column, $i$-PrOH/hexane 5:95; $1.0 \mathrm{~mL} / \mathrm{min}, 254 \mathrm{~nm}$ ); (3R) isomer (minor) $t_{\mathrm{R}}=4.87 \mathrm{~min}$ and $(3 S)$ isomer (major) $t_{\mathrm{R}}=5.55 \mathrm{~min} ;[\alpha]_{\mathrm{D}}=+69.6\left(\mathrm{c}=0.87, \mathrm{CHCl}_{3}\right)$; $\mathrm{mp} 60{ }^{\circ} \mathrm{C}$. Anal. Calcd. for $\mathrm{C}_{18} \mathrm{H}_{20} \mathrm{OSi}$ : C, 77.09; H, 7.19. Found: C, 77.36; H, 7.33. A copy of the NMR spectra is provided.

\section{(3R)-(2-Furanyl)indan-1-one (2i)}

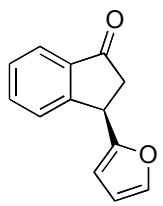

Compound 2i was prepared according to the general procedure (I) using (E)-2-[3-(furan-2-yl)prop-2enoyl]phenyl nonafluorobutanesulfonate $1 \mathbf{i}(248 \mathrm{mg}, 0.500 \mathrm{mmol}), \mathrm{Pd}(\mathrm{OAc})_{2}(5.6 \mathrm{mg}, 0.025 \mathrm{mmol})$,

$(R)-3,5$-XylMeOBIPHEP (35 mg, $0.050 \mathrm{mmol})$ and proton sponge $(214 \mathrm{mg}, 1.00 \mathrm{mmol})$ in DMF (2 $\mathrm{mL}$ ). The crude material was purified by column chromatography (hexane/ethyl acetate 5:1) to give the title compound as a pale yellow oil (72 mg, 73\%, 50\% ee). ${ }^{1} \mathrm{H}$ NMR $\left(400 \mathrm{MHz}, \mathrm{CDCl}_{3}\right) \delta 7.80(\mathrm{~d}, 1 \mathrm{H}, J=7.7 \mathrm{~Hz})$, $7.62(\mathrm{t}, 1 \mathrm{H}, J=7.8 \mathrm{~Hz}), 7.52(\mathrm{~d}, 1 \mathrm{H}, J=7.8 \mathrm{~Hz}), 7.44(\mathrm{t}, 1 \mathrm{H}, J=7.4 \mathrm{~Hz}), 7.35(\mathrm{~m}, 1 \mathrm{H}), 6.32(\mathrm{~m}, 1 \mathrm{H}), 6.11(\mathrm{~d}$, $1 \mathrm{H}, J=3.2 \mathrm{~Hz}), 4.69(\mathrm{dd}, 1 \mathrm{H}, J=4.0,8.0 \mathrm{~Hz}), 3.12(\mathrm{dd}, 1 \mathrm{H}, J=8.0,19.0 \mathrm{~Hz}), 2.87(\mathrm{dd}, 1 \mathrm{H}, J=4.0,19.0 \mathrm{~Hz})$; ${ }^{13} \mathrm{C}$ NMR $\left(100 \mathrm{MHz}, \mathrm{CDCl}_{3}\right) \delta 205.1,155.3,154.8,142.3,136.5,135.2,128.4,126.8,123.8,110.4,106.0,42.9$, 37.8; IR (neat) v 3410, 3118, 3074, 2924, 1714, 1605, 1506, 1463, 1405, 1320, 1287, 1240, 1173, 1151, 1093, $1045,1012,933,911,761,737 \mathrm{~cm}^{-1}$; the enantiomeric excess of $\mathbf{2} \mathbf{i}$ was determined by HPLC analysis $[(R, R)-$ Whelk-O 1 column, $i-\mathrm{PrOH} /$ hexane $1: 99 ; 1.0 \mathrm{~mL} / \mathrm{min}, 254 \mathrm{~nm}] ;(3 S)$ isomer (minor) $t_{\mathrm{R}}=32.6$ min and $(3 R)$ isomer (major) $t_{\mathrm{R}}=36.2 \mathrm{~min} ;[\alpha]_{\mathrm{D}}=-4.3\left(\mathrm{c}=0.6, \mathrm{CHCl}_{3}\right)$. A copy of the NMR spectra is provided.

\section{(3S)-Phenyl-benz(e)indan-1-one (2k)}

Compound 2k was prepared according to the general procedure (I) using (E)-1-(3-phenylprop-2enoyl)naphthalen-2-yl nonafluorobutanesulfonate $\mathbf{1 k}(278 \mathrm{mg}, 0.500 \mathrm{mmol}), \mathrm{Pd}(\mathrm{OAc})_{2}(5.6 \mathrm{mg}$, $0.025 \mathrm{mmol}),(R)-3,5-X y l M e O B I P H E P(35 \mathrm{mg}, 0.050 \mathrm{mmol})$ and proton sponge $(214 \mathrm{mg}, 1.00$ mmol) in DMF ( $2 \mathrm{~mL})$. The crude material was purified by column chromatography (hexane/ethyl acetate 7:1) to give the title compound as a yellowish solid (80 mg, 62\%, 64\% ee). $\left.{ }^{1} \mathrm{H} \mathrm{NMR} \mathrm{(400} \mathrm{MHz,} \mathrm{CDCl}_{3}\right) \delta$ $9.27(\mathrm{~d}, 1 \mathrm{H}, J=8.3 \mathrm{~Hz}), 8.01(\mathrm{~d}, 1 \mathrm{H}, J=8.4 \mathrm{~Hz}), 7.91(\mathrm{~d}, 1 \mathrm{H}, J=8.2 \mathrm{~Hz}), 7.74(\mathrm{t}, 1 \mathrm{H}, J=7.1 \mathrm{~Hz}), 7.62(\mathrm{t}, 1 \mathrm{H}, J$ $=8.1 \mathrm{~Hz}), 7.27-7.34(\mathrm{~m}, 4 \mathrm{H}), 7.17(\mathrm{~m}, 2 \mathrm{H}), 4.63(\mathrm{dd}, 1 \mathrm{H}, J=3.3 .7 .7 \mathrm{~Hz}), 3.36(\mathrm{dd}, 1 \mathrm{H}, J=7.7,19.0 \mathrm{~Hz}), 2.83$ $(\mathrm{dd}, 1 \mathrm{H}, J=3.3,19.0 \mathrm{~Hz}) ;{ }^{13} \mathrm{C} \mathrm{NMR}\left(100 \mathrm{MHz}, \mathrm{CDCl}_{3}\right) \delta 206.7,160.9,143.5,136.3,132.9,130.8,129.3,129.1$, 129.1, 128.3, 127.9, 127.2, 127.1, 124.4, 124.0, 47.6, 44.6; IR (neat) $v 3058,3027,1698,1626,1572,1512,1454$, 1438, 1377, 1269, 1177, 1108, 932, 832, 767, 755, 701, 511, $476 \mathrm{~cm}^{-1}$; Anal. Calcd. for $\mathrm{C}_{19} \mathrm{H}_{14} \mathrm{O}: \mathrm{C}, 88.34$; $\mathrm{H}$, 5.46. Found: $\mathrm{C}, 88.05 ; \mathrm{H}, 5.44 ; \mathrm{mp} 115^{\circ} \mathrm{C}$; the enantiomeric excess of $\mathbf{2 k}$ was determined by HPLC analysis (Chiracel OJ column, $i$-PrOH/hexane 5:95; $1.0 \mathrm{~mL} / \mathrm{min}, 254 \mathrm{~nm}$ ); $(3 R)$ isomer (minor) $t_{\mathrm{R}}=20.7 \mathrm{~min}$ and $(3 S$ ) isomer (major) $t_{\mathrm{R}}=22.8 \mathrm{~min} ;[\alpha]_{\mathrm{D}}=+146.2\left(\mathrm{c}=0.6, \mathrm{CHCl}_{3}\right)$. 


\section{(3R)-Ferrocenylindan-1-one (2l)}

Compound $2 \mathbf{l}$ was prepared according to the general procedure (I) using (E)-2-[3-(1-ferrocenyl)prop2-enoyl]phenyl nonafluorobutanesulfonate 11 (191 mg, $0.310 \mathrm{mmol}), \mathrm{Pd}(\mathrm{OAc})_{2}(3.6 \mathrm{mg}, 0.016$

$\mathrm{mmol}),(R)-3,5$-XylMeOBIPHEP (22 $\mathrm{mg}, 0.032 \mathrm{mmol}$ ) and proton sponge (137 $\mathrm{mg}, 0.640 \mathrm{mmol})$ in DMF (4 mL). The crude material was purified by column chromatography (hexane/ethyl acetate 5:1) to give the title compound as an orange solid (41 mg, 42\%, 88\% ee). ${ }^{1} \mathrm{H} \mathrm{NMR}\left(400 \mathrm{MHz}, \mathrm{CDCl}_{3}\right) \delta 7.75(\mathrm{~d}, 1 \mathrm{H}, J=7.7 \mathrm{~Hz})$, $7.57(\mathrm{t}, 1 \mathrm{H}, J=8 \mathrm{~Hz}), 7.45(\mathrm{~d}, 1 \mathrm{H}, J=7.7 \mathrm{~Hz}), 7.38(\mathrm{t}, 1 \mathrm{H}, J=7.5 \mathrm{~Hz}), 4.31(\mathrm{dd}, 1 \mathrm{H}, J=3.6,7.8 \mathrm{~Hz}), 4.24(\mathrm{~m}$, $1 \mathrm{H}), 4.16-4.21(\mathrm{~m}, 7 \mathrm{H}), 3.97(\mathrm{~m}, 1 \mathrm{H}), 3.21(\mathrm{dd}, 1 \mathrm{H}, J=7.8,19.1 \mathrm{~Hz}), 2.97(\mathrm{dd}, 1 \mathrm{H}, J=3.6,19.1 \mathrm{~Hz}) ;{ }^{13} \mathrm{C}$ NMR $\left(100 \mathrm{MHz}, \mathrm{CDCl}_{3}\right) \delta 206.3,158.2,136.2,135.0,128.0,126.5,123.7,91.0,68.8,68.5,67.9,65.5,45.4,38.4$; IR (neat) $v 3396,3071,1703,1596,1457,1405,1394,1323,1279,1235,1103,1044,1028,999,904,820,764,487$ $\mathrm{cm}^{-1}$; decomposition $>145^{\circ} \mathrm{C}$; the enantiomeric excess of $\mathbf{2 l}$ was determined by HPLC analysis $[(R, R)$-Whelk-O 1 column, $i-\mathrm{PrOH} /$ hexane 5:95; $1.0 \mathrm{~mL} / \mathrm{min}, 254 \mathrm{~nm}] ;(3 S)$ isomer (minor) $t_{\mathrm{R}}=15.8 \mathrm{~min}$ and $(3 R)$ isomer (major) $t_{\mathrm{R}}=22.0 \mathrm{~min} ;[\alpha]_{\mathrm{D}}=-118.9\left(\mathrm{c}=0.34, \mathrm{CHCl}_{3}\right)$. A copy of the NMR spectra is provided.

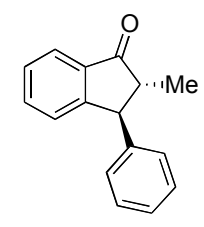

\section{$(2 R, 3 S)-2-M e t h y l-3-p h e n y l-i n d a n o n e ~(2 m)^{x x}$}

Compound $\mathbf{2 m}$ was prepared according to the general procedure (I) using (E)-2-(2-methyl-3phenylprop-2-enoyl)phenyl trifluoromethanesulfonate $\mathbf{1 m}(185 \mathrm{mg}, 0.500 \mathrm{mmol}), \mathrm{Pd}(\mathrm{OAc})_{2}(5.6$ $\mathrm{mg}, 0.025 \mathrm{mmol}),(R)-3,5$-XylMeOBIPHEP (35 mg, $0.05 \mathrm{mmol}$ ) and proton sponge (214 mg, 1.00 $\mathrm{mmol}$ ) in DMF (2 mL). The crude material was purified by column chromatography (hexane/ethyl acetate $7: 1)$ to give the title compound as a colorless oil $[91.4 \mathrm{mg}, 82 \%, d r=83: 17$ (the relative configuration was determined to be trans by NOE), 94\% ee (major enantiomer)]. Isomerization of this product was obtained by dissolving the product $(91.4 \mathrm{mg}, 0.412 \mathrm{mmol})$ in THF $(3 \mathrm{~mL})$ and adding $3 \mathrm{M} \mathrm{HCl}(4 \mathrm{~mL})$. The reaction mixture was stirred at 60 ${ }^{\circ} \mathrm{C}$ for $12 \mathrm{~h}$. The solution was cooled to room temperature and the organic phase was extracted with EtOAc $(2 \times 5$ $\mathrm{mL}$ ). The organic phase was washed with brine and dried over $\mathrm{MgSO}_{4}$. The solvent was removed under reduced pressure and the crude material was purified by column chromatography (hexane/ethyl acetate 7:1) to give the title compound as a colorless oil [77.8 $\mathrm{mg}, 85 \%, d r=91: 9,94 \%$ ee (major enantiomer)]. The enantiomeric excess of the major diastereomer $\mathbf{2 m}$ was determined by HPLC analysis (Chiracel OD-H column, $i$-PrOH/hexane 1:99; $0.8 \mathrm{~mL} / \mathrm{min}, 254 \mathrm{~nm}) ;(2 S, 3 R)$ isomer (minor) $t_{\mathrm{R}}=9.4 \mathrm{~min}$ and $(2 R, 3 S)$ isomer (major) $t_{\mathrm{R}}=10.2 \mathrm{~min} ;{ }^{1} \mathrm{H} \mathrm{NMR}$ $\left(400 \mathrm{MHz}, \mathrm{CDCl}_{3}\right) \delta 7.80(\mathrm{~m}, 1 \mathrm{H}), 7.54(\mathrm{~m}, 1 \mathrm{H}), 7.40(\mathrm{~m}, 1 \mathrm{H}), 7.32(\mathrm{~m}, 2 \mathrm{H}), 7.26(\mathrm{~m}, 1 \mathrm{H}), 7.19(\mathrm{~m}, 1 \mathrm{H}), 7.14$ $(\mathrm{m}, 2 \mathrm{H}), 4.00(\mathrm{~d}, 1 \mathrm{H}, \mathrm{J}=5.0 \mathrm{~Hz}), 2.62(\mathrm{~m}, 1 \mathrm{H}), 1.35(\mathrm{~d}, 3 \mathrm{H}, J=7.3 \mathrm{~Hz}) ;{ }^{13} \mathrm{C} \mathrm{NMR}\left(100 \mathrm{MHz}, \mathrm{CDCl}_{3}\right) \delta 208.0$, 156.2, 143.0, 136.4, 135.2, 129.1, 128.2, 128.1, 127.3, 126.7, 123.7, 54.0, 53.7, 14.3; IR (neat) $v 3028,2965$, 2930, 1716, 1604, 1494, 1454, 1320, 1206, 959, 742, $701 \mathrm{~cm}^{-1}$; Anal. Calcd. for $\mathrm{C}_{16} \mathrm{H}_{14} \mathrm{O}: \mathrm{C}, 86.45 ; \mathrm{H}, 6.35$. Found: C, 86.17; H, 6.42. 


\section{General procedure (II) for the reductive-Heck reaction with pentamethylpiperidine.}

An oven-dried Schlenk tube equipped with a Teflon screw seal was charged with a magnetic stirbar, the corresponding nonaflate or triflate (1 equiv), $\mathrm{Pd}(\mathrm{OAc})_{2}(5 \mathrm{~mol} \%),(R)-3,5-\mathrm{XylMeOBIPHEP}(10 \mathrm{~mol} \%)$ and pentamethylpiperidine ( 3 equiv). The tube was evacuated and backfilled with argon; this procedure was carried out two times. The solids were dissolved in the corresponding solvent ( $4 \mathrm{~mL} / \mathrm{mmol}$ nonaflate or triflate), the reaction tube was sealed and the reaction mixture was stirred in a pre-heated oil-bath at $100{ }^{\circ} \mathrm{C}$ for $12 \mathrm{~h}$. The reaction mixture was cooled to room temperature, diluted with ethyl acetate $(20 \mathrm{~mL} / \mathrm{mmol}$ nonaflate) and washed with an aqueous solution $\mathrm{HCl}(1 \mathrm{M}, 20 \mathrm{~mL} / \mathrm{mmol}$ nonaflate). The organic phase was separated, washed with brine and dried over $\mathrm{MgSO}_{4}$. The solvent was evaporated under reduced pressure and the crude material was purified by column chromatography on silica gel.

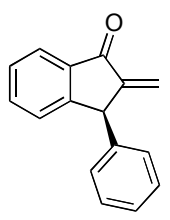

2-Methylene-(3S)-phenylindan-1-one (3a) ${ }^{\mathrm{xxi}}$

Compound 3a was prepared according to the general procedure (II) using (E)-2-(3-phenylprop-2enoyl)phenyl nonafluorobutanesulfonate 1a $(\mathrm{X}=\mathrm{ONf})(253 \mathrm{mg}, 0.500 \mathrm{mmol}), \mathrm{Pd}(\mathrm{OAc})_{2}(5.6 \mathrm{mg}$, $0.025 \mathrm{mmol}),(R)-3,5$-XylMeOBIPHEP (35 mg, $0.050 \mathrm{mmol})$ and pentamethylpiperidine $(270 \mu \mathrm{l}, 1.5$ $\mathrm{mmol})$ in 1,4-dioxane $(2 \mathrm{~mL})$. The crude material was purified by column chromatography (hexane/ethyl acetate 5:1) to give the title compound as a white solid (82 mg, 74\%, 80\% ee). ${ }^{1} \mathrm{H}$ NMR $\left(400 \mathrm{MHz}, \mathrm{CDCl}_{3}\right) \delta 7.91(\mathrm{~d}, 1 \mathrm{H}$, $J=7.7 \mathrm{~Hz}), 7.56(\mathrm{t}, 1 \mathrm{H}, J=7.4 \mathrm{~Hz}), 7.42(\mathrm{t}, 1 \mathrm{H}, J=7.5 \mathrm{~Hz}), 7.23-7.30(\mathrm{~m}, 4 \mathrm{H}), 7.09$ (d, 2H, $J=7.0 \mathrm{~Hz}), 6.40(\mathrm{~d}$, $1 \mathrm{H}, J=2.1 \mathrm{~Hz}), 5.41,(\mathrm{~d}, 1 \mathrm{H}, J=1.4 \mathrm{~Hz}), 5.00(\mathrm{~s}, 1 \mathrm{H}) ;{ }^{13} \mathrm{C} \mathrm{NMR}\left(100 \mathrm{MHz}, \mathrm{CDCl}_{3}\right) \delta 193.6,153.7,149.2,142.5$, 137.6, 135.6, 129.0, 128.5, 128.3, 127.3, 126.7, 124.4, 121.2, 49.3; IR (neat) v 3061, 3028, 1705, 1641, 1605, 1466, 1453, 1392, 1325, 1295, 1232, 1095, 984, 948, 740, $700 \mathrm{~cm}^{-1}$; mp $60{ }^{\circ} \mathrm{C}$; the enantiomeric excess of 3a was determined by HPLC analysis (Chiracel OJ column, $i$-PrOH/hexane 5:95; $1.0 \mathrm{~mL} / \mathrm{min}, 254 \mathrm{~nm}$ ); (3R) isomer (minor) $t_{\mathrm{R}}=8.5 \mathrm{~min}$ and $(3 S)$ isomer (major) $t_{\mathrm{R}}=13.5 \mathrm{~min} ;[\alpha]_{\mathrm{D}}=+74.7\left(\mathrm{c}=0.78, \mathrm{CHCl}_{3}\right.$ ); A copy of the NMR spectra is provided.

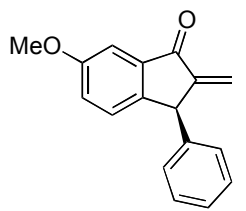

\section{6-Methoxy-2-methylene- (3S)-phenylindan-1-one (3b)}

Compound $\mathbf{3 b}$ was prepared according to the general procedure (II) using (E)-4-methoxy-2-(3phenylprop-2-enoyl)phenyl nonafluorobutanesulfonate $\mathbf{1 b}(268 \mathrm{mg}, 0.500 \mathrm{mmol}), \mathrm{Pd}(\mathrm{OAc})_{2}(5.6$ $\mathrm{mg}, 0.025 \mathrm{mmol}),(R)-3,5-\mathrm{Xyl}-\mathrm{MeO}-\mathrm{BIPHEP}(35 \mathrm{mg}, 0.050 \mathrm{mmol})$ and pentamethylpiperidine $(270 \mu \mathrm{l}, 1.5 \mathrm{mmol})$ in 1,4-dioxane $(2 \mathrm{~mL})$. The crude material was purified by column chromatography (hexane/ethyl acetate 5:1) to give the title compound as a white solid (82 mg, 65\%, 76\% ee). ${ }^{1} \mathrm{H} \mathrm{NMR}(400 \mathrm{MHz}$, $\left.\mathrm{CDCl}_{3}\right) \delta 7.32(\mathrm{~m}, 1 \mathrm{H}), 7.20-7.29(\mathrm{~m}, 3 \mathrm{H}), 7.15(\mathrm{~m}, 2 \mathrm{H}), 7.07-7.09(\mathrm{~m}, 2 \mathrm{H}), 6.36(\mathrm{dd}, 1 \mathrm{H}, J=0.5,2.2 \mathrm{~Hz}), 5.34$ 
$(\mathrm{dd}, 1 \mathrm{H}, J=0.4,1.7 \mathrm{~Hz}), 4.89(\mathrm{t}, 1 \mathrm{H}, J=1.9 \mathrm{~Hz}), 3.84(\mathrm{~s}, 3 \mathrm{H}) ;{ }^{13} \mathrm{C} \mathrm{NMR}\left(100 \mathrm{MHz}, \mathrm{CDCl}_{3}\right) \delta 193.5,160.0$, $149.8,146.6,142.7,138.9,128.9,128.3,127.5,127.2,125.0,121.0,105.3,55.75,48.5$; IR (neat) $v 3027,2941$, $2836,1704,1641,1612,1489,1331,1282,1251,1189,1159,1026,1008,837,800,762,700 \mathrm{~cm}^{-1} ; \mathrm{mp}^{\circ}{ }^{\circ} \mathrm{C}$; the enantiomeric excess of $\mathbf{3 b}$ was determined by HPLC analysis (Chiracel OJ column, $i$-PrOH/hexane 5:95; 1.0 $\mathrm{mL} / \mathrm{min}, 254 \mathrm{~nm}) ;(3 R)$ isomer (minor) $t_{\mathrm{R}}=11.8 \mathrm{~min}$ and $(3 S)$ isomer (major) $t_{\mathrm{R}}=15.0 \mathrm{~min} ;[\alpha]_{\mathrm{D}}=+71.0(\mathrm{c}=$ $0.86, \mathrm{CHCl}_{3}$ ); A copy of the NMR spectra is provided.

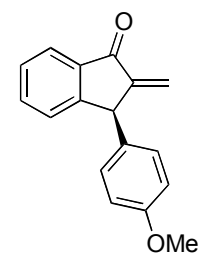

\section{(3S)-(4-Methoxyphenyl)-2-methylene-indan-1-one (3c)}

Compound 3c was prepared according to the general procedure (II) using (E)-2-[3-(4methoxyphenyl)prop-2-enoyl]phenyl nonafluorobutanesulfonate 1c (268 $\mathrm{mg}, 0.500 \mathrm{mmol})$, $\mathrm{Pd}(\mathrm{OAc})_{2}(5.6 \mathrm{mg}, 0.025 \mathrm{mmol}),(R)-3,5-\mathrm{Xyl}-\mathrm{MeO}-\mathrm{BIPHEP}(35 \mathrm{mg}, 0.050 \mathrm{mmol})$ and pentamethylpiperidine $(270 \mu \mathrm{l}, 1.5 \mathrm{mmol})$ in 1,4-dioxane $(2 \mathrm{~mL})$. The crude material was purified by column chromatography (hexane/ethyl acetate 5:1) to give the title compound as a yellow solid (91 mg, 73\%, 74\% ee). ${ }^{1} \mathrm{H}$ NMR (400 MHz, $\left.\mathrm{CDCl}_{3}\right) \delta 7.91(\mathrm{~d}, 1 \mathrm{H}, J=7.7 \mathrm{~Hz}), 7.58(\mathrm{t}, 1 \mathrm{H}, J=7.4 \mathrm{~Hz}), 7.43(\mathrm{t}, 1 \mathrm{H}, J=7.6 \mathrm{~Hz}), 7.30(\mathrm{~d}, 2 \mathrm{H}$, $J=7.7 \mathrm{~Hz}), 7.04(\mathrm{~d}, 1 \mathrm{H}, J=8.4 \mathrm{~Hz}), 6.85(\mathrm{~d}, 2 \mathrm{H}, J=8.3 \mathrm{~Hz}), 6.41(\mathrm{~d}, 2 \mathrm{H}, J=2.0 \mathrm{~Hz}), 5.43(\mathrm{~s}, 1 \mathrm{H}), 4.95(\mathrm{~s}, 1 \mathrm{H})$, $3.78(\mathrm{~s}, 3 \mathrm{H}) ;{ }^{13} \mathrm{C} \mathrm{NMR}\left(100 \mathrm{MHz}, \mathrm{CDCl}_{3}\right) \delta 193.6,158.7,154.0,149.5,137.5,135.5,134.5,129.4,128.2,126.6$, 124.2, 121.0, 114.3, 55.3, 48.4; IR (neat) v 3032, 3000, 2955, 2836, 1705, 1640, 1606, 1511, 1466, 1396, 1327, 1297, 1251, 1177, 1110, 1095, 1034, 984, 950, 826, $746 \mathrm{~cm}^{-1}$; mp 45-48 ${ }^{\circ} \mathrm{C}$; the enantiomeric excess of $\mathbf{3 c}$ was determined by HPLC analysis (Chiracel OJ column, $i$-PrOH/hexane 5:95; $1.0 \mathrm{~mL} / \mathrm{min}, 254 \mathrm{~nm}$ ); $(3 R$ ) isomer (minor) $t_{\mathrm{R}}=15.0 \mathrm{~min}$ and $(3 S)$ isomer (major) $t_{\mathrm{R}}=33.8 \mathrm{~min} ;[\alpha]_{\mathrm{D}}=+72.6\left(\mathrm{c}=0.9, \mathrm{CHCl}_{3}\right)$; A copy of the NMR spectra is provided.

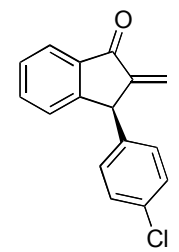

\section{(3S)-(4-Chloroyphenyl)-2-methylene-indan-1-one (3d)}

Compound 3d was prepared according to the general procedure (II) using (E)-2-[3-(4Chloroyphenyl)prop-2-enoyl]phenyl nonafluorobutanesulfonate $1 \mathbf{d}(270 \mathrm{mg}, 0.500 \mathrm{mmol}), \mathrm{Pd}(\mathrm{OAc})_{2}$ (5.6 mg, $0.025 \mathrm{mmol}),(R)-3,5-\mathrm{Xyl}-\mathrm{MeO}-\mathrm{BIPHEP}(35 \mathrm{mg}, 0.050 \mathrm{mmol})$ and pentamethylpiperidine

$(270 \mu \mathrm{l}, 1.5 \mathrm{mmol})$ in 1,4-dioxane $(2 \mathrm{~mL})$. The crude material was purified by column chromatography (hexane/ethyl acetate 7:1) to give the title compound as a pale-yellow solid (57 mg, 44\%, 80\% ee). ${ }^{1} \mathrm{H}$ NMR (400 $\left.\mathrm{MHz}, \mathrm{CDCl}_{3}\right) \delta 7.88(\mathrm{t}, 1 \mathrm{H}, J=7.7 \mathrm{~Hz}), 7.57(\mathrm{t}, 1 \mathrm{H}, J=7.4 \mathrm{~Hz}), 7.43(\mathrm{t}, 1 \mathrm{H}, J=7.6 \mathrm{~Hz}), 7.22-7.26(\mathrm{~m}, 3 \mathrm{H}), 7.03$ $(\mathrm{d}, 2 \mathrm{H}, J=8.2 \mathrm{~Hz}), 6.40(\mathrm{~d}, 1 \mathrm{H}, J=2.3 \mathrm{~Hz}), 5.39(\mathrm{~d}, 1 \mathrm{H}, J=1.8 \mathrm{~Hz}), 4.94(\mathrm{~s}, 1 \mathrm{H}) ;{ }^{13} \mathrm{C} \mathrm{NMR}\left(100 \mathrm{MHz}, \mathrm{CDCl}_{3}\right)$ $\delta 193.3,153.2,148.9,141.1,137.6,135.8,133.2,129.9,129.2,128.6,126.6,124.6,121.5,48.6$; IR (neat) $v 3068$, 1708, 1638, 1605, 1491, 1466, 1409, 1394, 1330, 1297, 1229, 1090, 1015, 984, 952, 817, 746, $511 \mathrm{~cm}^{-1}$; Anal. Calcd. for $\mathrm{C}_{16} \mathrm{H}_{11} \mathrm{ClO}$ : C, 75.45; H, 4.35. Found: C, 75.17; H, 4.45; the enantiomeric excess of 3d was determined 
by HPLC analysis (Chiracel OJ column, $i-\mathrm{PrOH} /$ hexane 5:95; $1.0 \mathrm{~mL} / \mathrm{min}, 254 \mathrm{~nm}$ ); $(3 R)$ isomer (minor) $t_{\mathrm{R}}=$ $11.0 \mathrm{~min}$ and $(3 S)$ isomer (major) $t_{\mathrm{R}}=15.3 \mathrm{~min} ;[\alpha]_{\mathrm{D}}=+81.5\left(\mathrm{c}=0.83, \mathrm{CHCl}_{3}\right) ; \mathrm{mp} 56-60 \mathrm{C}$. A copy of the NMR spectra is provided.

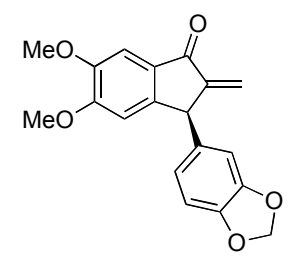

\section{(3S)-(3,4-Methylendioxyphenyl)-5,6-dimethoxy-2-methylene-indan-1-one (3e)}

Compound 3e was prepared according to the general procedure (II) using (E)-2-(3-(3,4methylendioxy)prop-2-enoyl)-4,5-dimethoxyphenyl trifluoromethanesulfonate 1n (305 mg, $0.500 \mathrm{mmol}), \mathrm{Pd}(\mathrm{OAc})_{2}(5.6 \mathrm{mg}, 0.025 \mathrm{mmol}),(R)-3,5$-XylMeOBIPHEP (35 mg, 0.050 $\mathrm{mmol})$ and pentamethylpiperidine $(270 \mu \mathrm{l}, 1.5 \mathrm{mmol})$ in 1,4-dioxane $(2 \mathrm{~mL})$. The crude material was purified by column chromatography (hexane/ethyl acetate 1:1) to give the title compound as a light-yellow solid (118 mg, $73 \%, 70 \%$ ee). ${ }^{1} \mathrm{H}$ NMR (400 MHz, $\left.\mathrm{CDCl}_{3}\right) \delta 7.29$ (s, 1H), 6.75 (d, 1H, J=7.9 Hz), 6.65 (dd, $1 \mathrm{H}, J=1.7,7.9$ $\mathrm{Hz}), 6.64(\mathrm{~s}, 1 \mathrm{H}), 6.42(\mathrm{~d}, 1 \mathrm{H}, J=1.7 \mathrm{~Hz}), 6.28(\mathrm{~d}, 1 \mathrm{H}, J=2.1 \mathrm{~Hz}), 5.90(\mathrm{~s}, 1 \mathrm{H}), 5.30(\mathrm{~m}, 1 \mathrm{H}), 4.77(\mathrm{~s}, 1 \mathrm{H}), 3.93$ $(\mathrm{s}, 3 \mathrm{H}), 3.85(\mathrm{~s}, 3 \mathrm{H}) ;{ }^{13} \mathrm{C} \mathrm{NMR}\left(100 \mathrm{MHz}, \mathrm{CDCl}_{3}\right) \delta 192.2,156.3,150.3,149.8,149.0,148.3,146.9,136.4$, 131.0, 121.8, 119.5, 108.5, 108.4, 107.2, 104.7, 101.3, 56.6, 56.4, 48.7; IR (neat) v2939, 1734, 1694, 1642, 1586, $1489,1442,1361,1304,1241,1111,1037,1018,035,868,794 \mathrm{~cm}^{-1} ; \mathrm{mp} \mathrm{53-55}{ }^{\circ} \mathrm{C}$; the enantiomeric excess of $3 \mathbf{e}$ was determined by HPLC analysis (Chiracel OD column, $i$-PrOH/hexane 10:90; $1.0 \mathrm{~mL} / \mathrm{min}, 254 \mathrm{~nm}) ;(3 S)$ isomer (major) $t_{\mathrm{R}}=14.5 \mathrm{~min}$ and $(3 R)$ isomer (minor) $t_{\mathrm{R}}=17.9 \mathrm{~min} ;[\alpha]_{\mathrm{D}}=-12.6\left(\mathrm{c}=0.92, \mathrm{CHCl}_{3}\right)$; A copy of the NMR spectra is provided.

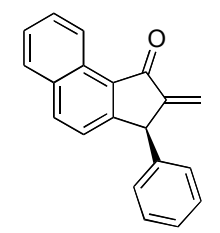

\section{2-Methylene-(3S)-phenyl-benz(e)indan-1-one (3f)}

Compound $3 \mathbf{f}$ was prepared according to the general procedure (II) using (E)-1-(3-phenylprop-2enoyl)naphthalen-2-yl nonafluorobutanesulfonate $\mathbf{1 k}(278 \mathrm{mg}, 0.500 \mathrm{mmol}), \mathrm{Pd}(\mathrm{OAc})_{2}(5.6 \mathrm{mg}$, $0.025 \mathrm{mmol}),(R)-3,5$-XylMeOBIPHEP $(35 \mathrm{mg}, 0.050 \mathrm{mmol})$ and pentamethylpiperidine $(270 \mu \mathrm{l}$, $1.5 \mathrm{mmol})$ in 1,4-dioxane $(2 \mathrm{~mL})$. The crude material was purified by column chromatography (hexane/ethyl acetate 5:1) to give the title compound as a beige solid (89 mg, 66\%, 60\% ee). ${ }^{1} \mathrm{H} \mathrm{NMR}\left(400 \mathrm{MHz}, \mathrm{CDCl}_{3}\right) \delta 9.32$ $(\mathrm{d}, 1 \mathrm{H}, J=8.3 \mathrm{~Hz}), 8.00(\mathrm{~d}, 1 \mathrm{H}, J=8.4 \mathrm{~Hz}), 7.87(\mathrm{~d}, 1 \mathrm{H}, J=8.1 \mathrm{~Hz}), 7.72(\mathrm{t}, 1 \mathrm{H}, J=7.3 \mathrm{~Hz}), 7.58(\mathrm{t}, 1 \mathrm{H}, J=7.3$ $\mathrm{Hz}), 7.23-7.31(\mathrm{~m}, 4 \mathrm{H}), 7.12(\mathrm{~m}, 2 \mathrm{H}), 6.41(\mathrm{~d}, 1 \mathrm{H}, J=1.8 \mathrm{~Hz}), 5.41(\mathrm{~s}, 1 \mathrm{H}), 5.04(\mathrm{~s}, 1 \mathrm{H}) ;{ }^{13} \mathrm{C} \mathrm{NMR}(100 \mathrm{MHz}$, $\left.\mathrm{CDCl}_{3}\right) \delta 194.2,156.1,149.6,142.1,136.8,133.1,132.1,129.6,129.5,128.6,127.4,127.3,124.8,123.6,120.0$, 49.4; IR (neat) v 3030, 1688, 1640, 1590, 1569, 1516, 1495, 1456, 1438, 1386, 1171, 1091, 932, 826, 789, 751, 700, $495 \mathrm{~cm}^{-1}$; Anal. Calcd. for $\mathrm{C}_{20} \mathrm{H}_{14} \mathrm{O}: \mathrm{C}, 88.86$; H, 5.22. Found: C, 86.55; H, 5.07; mp $140{ }^{\circ} \mathrm{C}$; the enantiomeric excess of $\mathbf{3 h}$ was determined by HPLC analysis (Chiracel OJ column, $i$-PrOH/hexane 5:95; 1.0 $\mathrm{mL} / \mathrm{min}, 254 \mathrm{~nm}$ ); $(3 R)$ isomer (minor) $t_{\mathrm{R}}=16.3 \mathrm{~min}$ and $(3 S)$ isomer (major) $t_{\mathrm{R}}=36.6 \mathrm{~min} ;[\alpha]_{\mathrm{D}}=+139.5(\mathrm{c}=$ $\left.0.8, \mathrm{CHCl}_{3}\right)$. A copy of the NMR spectra is provided. 


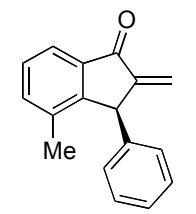

\section{4-Methyl-2-methylene-(3S)-phenylindan-1-one (3g)}

Compound 3g was prepared according to the general procedure (II) using (E)-2-methyl-6-(3phenylprop-2-enoyl)phenyl trifluoromethanesulfonate $1 \mathrm{~g}$ (185 mg, $0.500 \mathrm{mmol}), \operatorname{Pd}(\mathrm{OAc})_{2}(5.6 \mathrm{mg}$, $0.025 \mathrm{mmol}),(R)-3,5$-XylMeOBIPHEP $(35 \mathrm{mg}, 0.050 \mathrm{mmol})$ and pentamethylpiperidine $(270 \mu 1,1.5$ $\mathrm{mmol}$ ) in 1,4-dioxane $(2 \mathrm{~mL})$. The crude material was purified by column chromatography (hexane/ethyl acetate $7: 1)$ to give the title compound as a pale-yellow solid (83 mg, 70\%, 94\% ee). ${ }^{1} \mathrm{H}$ NMR $\left(400 \mathrm{MHz}, \mathrm{CDCl}_{3}\right) \delta 7.77$ $(\mathrm{m}, 1 \mathrm{H}), 7.36-7.38(\mathrm{~m}, 2 \mathrm{H}), 7.17-7.26(\mathrm{~m}, 3 \mathrm{H}), 7.01-7.02(\mathrm{~m}, 2 \mathrm{H}), 6.33(\mathrm{~d}, 1 \mathrm{H}, J=1.9 \mathrm{~Hz}), 5.37,(\mathrm{~d}, 1 \mathrm{H}, J=1.4$ $\mathrm{Hz}), 4.92$ (s, 1H), 1.97 (s, 3H); ${ }^{13} \mathrm{C}$ NMR (100 MHz, $\left.\mathrm{CDCl}_{3}\right) \delta 193.9,151.2,149.6,142.4,138.3,137.1,136.7$, 129.0, 128.7, 127.9, 127.1, 121.9, 120.9, 48.8, 18.7; IR (neat) v 3026, 1702, 1639, 1601, 1588, 1481, 1452, 1395, 1281, 1250, 1028, 987, 952, 763, 718, $699 \mathrm{~cm}^{-1} ; \mathrm{mp} 98^{\circ} \mathrm{C}$; the enantiomeric excess of $\mathbf{3 f}$ was determined by HPLC analysis (Chiracel OJ column, $i$-PrOH/hexane 5:95; $0.5 \mathrm{~mL} / \mathrm{min}, 254 \mathrm{~nm}$ ); (3R) isomer (major) $t_{\mathrm{R}}=7.2$ min and $(3 S)$ isomer (minor) $t_{\mathrm{R}}=9.1 \mathrm{~min} ;[\alpha]_{\mathrm{D}}=+48.4\left(\mathrm{c}=1.2, \mathrm{CHCl}_{3}\right)$. A copy of the NMR spectra is provided.

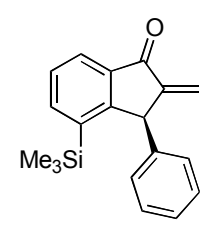

\section{2-Methylene-4-trimethylsilyl-(3R)-phenylindan-1-one (3h)}

Compound $3 \mathbf{h}$ was prepared according to the general procedure (II) using (E)-2'(trifluoromethanesulfonyl)-3'-trimethylsilylchalcone $\mathbf{1 h}$ (129 $\mathrm{mg}, 0.300 \mathrm{mmol}), \mathrm{Pd}(\mathrm{OAc})_{2}(3.4$ $\mathrm{mg}, 0.015 \mathrm{mmol}),(R)-3,5-X y l M e O B I P H E P(21 \mathrm{mg}, 0.030 \mathrm{mmol})$ and pentamethylpiperidine (163

$\mu \mathrm{l}, 0.900 \mathrm{mmol})$ in 1,4-dioxane $(1.2 \mathrm{~mL})$. The crude material was purified by column chromatography (hexane/ethyl acetate 7:1) to give the title compound as a pale-yellow oil (41 mg, 46\%, 90\% ee). ${ }^{1} \mathrm{H}$ NMR (400 $\left.\mathrm{MHz}, \mathrm{CD}_{2} \mathrm{Cl}_{2}\right) \delta 7.89(\mathrm{~m}, 1 \mathrm{H}), 7.51(\mathrm{t}, 1 \mathrm{H}, J=7.6 \mathrm{~Hz}), 7.20-7.29(\mathrm{~m}, 3 \mathrm{H}), 6.97(\mathrm{~m}, 2 \mathrm{H}), 6.24(\mathrm{~d}, 1 \mathrm{H}, J=2.0 \mathrm{~Hz})$, $5.41(\mathrm{~d}, 1 \mathrm{H}, J=1.6 \mathrm{~Hz}), 5.17,(\mathrm{~s}, 1 \mathrm{H}), 4.92(\mathrm{~s}, 1 \mathrm{H}),-0.01(\mathrm{~s}, 9 \mathrm{H}),{ }^{13} \mathrm{C} \mathrm{NMR}\left(100 \mathrm{MHz}, \mathrm{CD}_{2} \mathrm{Cl}_{2}\right) \delta 193.9,158.5$, $150.1,144.6,142.9,139.6,138.4,129.4,128.7,128.3,127.6,125.0,119.8,50.5,-0.4$; IR (neat) $v 3061,3027$, $2964,1705,1640,1587,1569,1494,1453,1467,1402,1282,1249,1001,937,839,755,715,700 \mathrm{~cm}^{-1}$; the enantiomeric excess of $\mathbf{3 g}$ was determined by HPLC analysis (Chiracel OJ column, $i$-PrOH/hexane 5:95; 1.0 $\mathrm{mL} / \mathrm{min}, 254 \mathrm{~nm}) ;(3 R)$ isomer (major) $t_{\mathrm{R}}=4.86 \mathrm{~min}$ and $(3 S)$ isomer (minor) $t_{\mathrm{R}}=6.31 \mathrm{~min} ;[\alpha]_{\mathrm{D}}=+151.9(\mathrm{c}=$ 0.73, $\mathrm{CHCl}_{3}$ ). Anal. Calcd. for $\mathrm{C}_{19} \mathrm{H}_{20} \mathrm{OSi}$ : C, 78.03; H, 6.89. Found: C, 78.31; H, 7.05. A copy of the NMR spectra is provided.

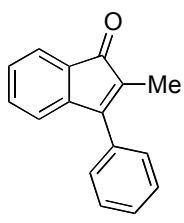

\section{2-Methyl-3-phenylinden-1-one (3i) ${ }^{\text {xii }}$}

Compound 3i was prepared according to the general procedure (II) using (E)-2-(3-phenylprop-2enoyl)phenyl nonafluorobutanesulfonate 1a $(\mathrm{X}=\mathrm{ONf})(253 \mathrm{mg}, 0.500 \mathrm{mmol}), \mathrm{Pd}(\mathrm{OAc})_{2}(5.6 \mathrm{mg}$, $0.025 \mathrm{mmol}),(R)-3,5-\mathrm{Xyl}-\mathrm{MeO}-\mathrm{BIPHEP}(35 \mathrm{mg}, 0.050 \mathrm{mmol})$ and pentamethylpiperidine $(270 \mu \mathrm{l}$, 
$1.5 \mathrm{mmol}$ ) in DMF (2 mL). The crude material was purified by column chromatography (hexane/ethyl acetate 5:1) to give the title compound as an orange solid $(77 \mathrm{mg}, 70 \%) .{ }^{1} \mathrm{H}$ NMR $\left(400 \mathrm{MHz}, \mathrm{CDCl}_{3}\right) \delta 7.41-7.51(\mathrm{~m}$, $6 \mathrm{H}), 7.26(\mathrm{td}, 1 \mathrm{H}, J=7.7,1.2 \mathrm{~Hz}), 7.16(\mathrm{t}, 1 \mathrm{H}, J=7.3 \mathrm{~Hz}), 7.02,(\mathrm{~d}, 1 \mathrm{H}, J=7.0 \mathrm{~Hz}), 1.90(\mathrm{~s}, 3 \mathrm{H}) ;{ }^{13} \mathrm{C} \mathrm{NMR}(100$ $\left.\mathrm{MHz}, \mathrm{CDCl}_{3}\right) \delta 198.5,154.8,145.8,133.3,132.9,131.3,131.2,129.4,128.9,128.2,128.2,122.7,120.6,8.8$; IR (neat) $v$ 3057, 2916, 1705, 1608, 1455, 1365, 1332, 1392, 1206, 1160, 1080, 1012, 934, 774, $701 \mathrm{~cm}^{-1}$; Anal. Calcd. for $\mathrm{C}_{16} \mathrm{H}_{12} \mathrm{O}: \mathrm{C}, 87.25 ; \mathrm{H}, 5.49$. Found: C, 86.97; H, 5.50; mp $82{ }^{\circ} \mathrm{C}$ (lit. ${ }^{22} \mathrm{mp} 83-84{ }^{\circ} \mathrm{C}$ ).

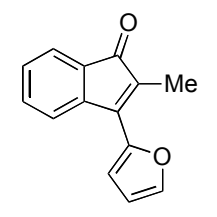

\section{3-(2-Furanyl)-2-methyl-inden-1-one (3k)}

Compound 3k was prepared according to the general procedure (II) using (E)-2-[3-(Furan-2yl)prop-2-enoyl]phenyl nonafluorobutanesulfonate 1i (248 mg, $0.500 \mathrm{mmol}), \mathrm{Pd}(\mathrm{OAc})_{2}(5.6 \mathrm{mg}$, $0.025 \mathrm{mmol}),(R)-3,5$-XylMeOBIPHEP $(35 \mathrm{mg}, 0.050 \mathrm{mmol})$ and pentamethylpiperidine $(270 \mu \mathrm{l}$, $1.5 \mathrm{mmol})$ in 1,4-dioxane $(2 \mathrm{~mL})$. The crude material was purified by column chromatography (hexane/ethyl acetate 5:1) to give the title compound as a red solid (77 mg, 73\%). ${ }^{1} \mathrm{H}$ NMR (400 MHz, $\left.\mathrm{CDCl}_{3}\right) \delta 7.65-7.67(\mathrm{~m}$, 2H), $7.43(\mathrm{~m}, 1 \mathrm{H}), 7.31(\mathrm{td}, 1 \mathrm{H}, J=7.6,1.3 \mathrm{~Hz}), 7.17(\mathrm{td}, 1 \mathrm{H}, J=7.9,0.8 \mathrm{~Hz}), 6.93(\mathrm{~d}, 1 \mathrm{H}, J=3.6 \mathrm{~Hz}), 6.61$ (dd, $1 \mathrm{H}, J=1.8,3.6 \mathrm{~Hz}), 2.05(\mathrm{~s}, 3 \mathrm{H}) ;{ }^{13} \mathrm{C} \mathrm{NMR}\left(100 \mathrm{MHz}, \mathrm{CDCl}_{3}\right) \delta 197.2,149.7,144.6,143.4,141.9,133.2,131.7$, 128.3, 128.3, 122.4, 122.3, 115.4, 112.7, 9.7; IR (neat) v 3374, 3139, 3068, 2924, 2853, 1695, 1602, 1582, 1478, 1324, 1219. 1151, 1034, 1013, 959, 741, $709 \mathrm{~cm}^{-1}$; mp 58-60 ${ }^{\circ} \mathrm{C}$; A copy of the NMR spectra is provided.

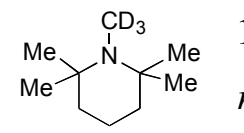

\section{1-(Trideuteromethyl)-2,2,6,6-tetramethylpiperidine (CD -PMP) $_{3}$}

$n$-BuLi (1.6 M in hexane; $11.3 \mathrm{~mL}, 15 \mathrm{mmol}$ ) was added dropwise to a solution of 2,2,6,6tetramethylpiperidine $(2.53 \mathrm{~mL}, 15.0 \mathrm{mmol})$ in $\mathrm{THF}$ at $-78^{\circ} \mathrm{C}$. After the addition was completed the reaction mixture was allowed to warm to room temperature and stirring was continued for further $20 \mathrm{~min}$ at that temperature. The reaction was cooled again to $-78{ }^{\circ} \mathrm{C}$ and $\mathrm{CD}_{3} \mathrm{I}(1.24 \mathrm{~mL}, 20.0 \mathrm{mmol})$ was added dropwise. After the addition was completed the reaction mixture was allowed to warm to room temperature and stirring was continued for further $2 \mathrm{~h}$ at that temperature. The solvent was removed under reduced pressure and the remaining residue was dissolved in $\mathrm{Et}_{2} \mathrm{O}(50 \mathrm{~mL}) . \mathrm{H}_{2} \mathrm{O}(50 \mathrm{~mL})$ was carefully added; the aqueous phase was separated and extracted with $\mathrm{Et}_{2} \mathrm{O}(2 \times 10 \mathrm{~mL})$. The combined organic phases were dried over $\mathrm{MgSO}_{4}$. Purification of the crude material by distillation under reduced pressure yielded the desired product as a colorless liquid $(2.0 \mathrm{~g}, 84 \%)$. ${ }^{1} \mathrm{H}$ NMR (400 MHz, $\left.\mathrm{C}_{6} \mathrm{D}_{6}\right) \delta 1.43(\mathrm{~s}, 6 \mathrm{H}), 1.02(\mathrm{~s}, 12 \mathrm{H}) ;{ }^{2} \mathrm{H}$ NMR (500 MHz, $\left.\mathrm{CH}_{2} \mathrm{Cl}_{2} / \mathrm{CD}_{2} \mathrm{Cl}_{2}\right) \delta 2.17(\mathrm{~s}, 3 \mathrm{H})$; IR (neat) $v 2965,2928,2190,2058,1467,1376,1361,1260,1141,1062,1019,977,949,875,826,626,519 \mathrm{~cm}^{-1}$; bp $70{ }^{\circ} \mathrm{C}(17 \mathrm{~mm} \mathrm{Hg})$. A copy of the ${ }^{1} \mathrm{H}$ NMR spectrum is provided. 


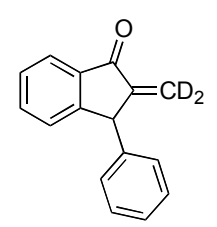

\section{2-(Dideuteromethylene)-3-phenylindan-1-one (4)}

Compound 4 was prepared according to the general procedure (II) using (E)-2-(3-phenylprop-2enoyl)phenyl nonafluorobutanesulfonate $(253 \mathrm{mg}, 0.500 \mathrm{mmol}), \mathrm{Pd}(\mathrm{OAc})_{2}(5.6 \mathrm{mg}, 0.025 \mathrm{mmol})$, $(R)-3,5-X y l-M e O-B I P H E P \quad(35 \quad \mathrm{mg}, \quad 0.050 \quad \mathrm{mmol}) \quad$ and 1-(trideuteromethyl)-2,2,6,6tetramethylpiperidine $(237 \mathrm{mg}, 1.50 \mathrm{mmol})$ in 1,4-dioxane $(2 \mathrm{~mL})$. After $12 \mathrm{~h}$ reaction time complete conversion of the starting material was observed, based on GC analysis. The crude material was purified by column chromatography (hexane/ethyl acetate $7: 1$ ) to give the title compound as a light-yellow solid (50 mg, 45\%). ${ }^{1} \mathrm{H}$ NMR (400 MHz, $\left.\mathrm{CD}_{2} \mathrm{Cl}_{2}\right) \delta 7.87(\mathrm{~d}, 1 \mathrm{H}, J=7.7 \mathrm{~Hz}), 7.61(\mathrm{td}, 1 \mathrm{H}, J=8.7,1.3 \mathrm{~Hz}), 7.47(\mathrm{~m}, 1 \mathrm{H}), 7.24-7.34(\mathrm{~m}$, 4H), $7.14(\mathrm{~m}, 2 \mathrm{H}), 5.03(\mathrm{~s}, 1 \mathrm{H}) ;{ }^{2} \mathrm{H}$ NMR $\left(500 \mathrm{MHz}, \mathrm{CH}_{3} \mathrm{CN} / \mathrm{CD}_{3} \mathrm{CN}\right) \delta 6.32(\mathrm{~s}, 1 \mathrm{H}), 5.44(\mathrm{~s}, 1 \mathrm{H}) ;{ }^{13} \mathrm{C} \mathrm{NMR}(100$ $\left.\mathrm{MHz}, \mathrm{CDCl}_{3}\right) \delta 193.6,153.8,149.1,142.5,137.7,135.6,129.0,128.5,128.3,127.3,126.7,124.4,49.2$; IR (neat) $v 3028,1703,1602,1466,1453,1324,1294,1232,1095,1060,945,741,725,699 \mathrm{~cm}^{-1}$; mp 64-68 ${ }^{\circ} \mathrm{C}$. A copy of the NMR spectra is provided.

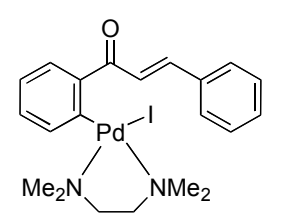

\section{PdI(chalcone)(tmeda)-complex}

A solution of $(E)-2^{\prime}$-iodo-chalcone $(2.66 \mathrm{~g}, 8 \mathrm{mmol}), \mathrm{Pd}(\mathrm{dba})_{2}(4.6 \mathrm{~g}, 8.0 \mathrm{mmol})$ and tmeda $(1.56 \mathrm{~mL}, 10.4 \mathrm{mmol})$ in THF $(40 \mathrm{~mL})$ was stirred for $3 \mathrm{~h}$ at $40^{\circ} \mathrm{C}$. The reaction mixture was cooled to room temperature and filtered over a plug of Celite ${ }^{\circledR}$. The solvent was removed under reduced pressure to yield an orange solid. The residue was washed with $\mathrm{Et}_{2} \mathrm{O}(4 \times 40 \mathrm{~mL})$ to remove dba. The solid was dried under reduced pressure to yield the desired product as a yellow powder ( $2.69 \mathrm{~g}, 60 \%)$, which appeared indefinitely stable at room temperature. ${ }^{1} \mathrm{H}$ NMR $\left(400 \mathrm{MHz}, \mathrm{CD}_{2} \mathrm{Cl}_{2}\right) \delta 8.69(\mathrm{~d}, 1 \mathrm{H}, J=15.7 \mathrm{~Hz}), 7.80$ $(\mathrm{m}, 2 \mathrm{H}), 7.71(\mathrm{~d}, 1 \mathrm{H}, J=15.7 \mathrm{~Hz}), 7.64(\mathrm{dd}, 1 \mathrm{H}, J=1.1,7.8 \mathrm{~Hz}), 7.52(\mathrm{dd}, 1 \mathrm{H}, J=1.6,7.6 \mathrm{~Hz}), 7.37-7.43$ (m, $3 \mathrm{H}), 7.05(\mathrm{~m}, 1 \mathrm{H}), 6.94(\mathrm{~m}, 1 \mathrm{H}), 2.18-2.64(\mathrm{~m}, 16 \mathrm{H}) ;{ }^{13} \mathrm{C} \mathrm{NMR}\left(125 \mathrm{MHz}, \mathrm{CD}_{3} \mathrm{CN}\right) \delta 194.5,150.0,146.7,140.2$, 139.0, 135.9, 130.2, 129.1, 129.0, 128.9, 128.6, 128.0, 122.8, 62.2, 58.2, 49.7, 49.4; IR (neat) v 3053, 2974, 2888, 2840, 2791, 1650, 1595, 1574, 1461, 1421, 1332, 1284, 1242, 1205, 1058, 1016, 979, 056, 802, 753, $733 \mathrm{~cm}^{-1}$; Anal. Calcd. for $\mathrm{C}_{21} \mathrm{H}_{27} \mathrm{IN}_{2} \mathrm{OPd}$ : C, 45.30; H, 4.89. Found: C, 45.60; H, 4.99; decomposition $>120^{\circ} \mathrm{C}$.

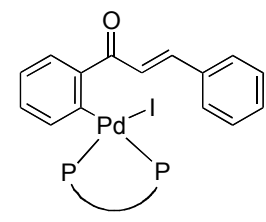

\section{PdI(chalcone)(rac-MeOBIPHEP)-complex}

A solution of the PdI(chalcone)(tmeda)-complex (353 mg, $0.630 \mathrm{mmol})$ and rac-MeOBIPHEP $(370 \mathrm{mg}, 0.63 \mathrm{mmol})$ in THF $(15 \mathrm{~mL})$ was stirred for $16 \mathrm{~h}$ at $40{ }^{\circ} \mathrm{C}$. The reaction mixture was cooled to room temperature and filtered over a plug of Celite ${ }^{\circledR}$. The solvent was removed under reduced pressure. The residue is dissolved in a minimal amount of toluene (approx. $5 \mathrm{~mL}$ ) and triturated with hexane (approx. $10 \mathrm{~mL}$ ) to precipitate the desired product. The solid is filtered off and washed with hexane $(4 \times 3 \mathrm{~mL})$. The desired product is obtained as a light-brown powder $(627 \mathrm{mg}, 95 \%)$, which appeared indefinitely 
stable at room temperature after drying under reduced pressure. ${ }^{1} \mathrm{H}$ NMR $\left(400 \mathrm{MHz}, \mathrm{CD}_{2} \mathrm{Cl}_{2}\right) \delta 7.85(\mathrm{~m}, 3 \mathrm{H})$, 7.61-7.74 (m, 7H), 7.35-7.49 (m, 10H), 7.09-7.22 (m, 10H), 6.94-7.02 (m, 2H), 6.83-6.94 (m, 3H), 6.48 (m, 1H), $6.37(\mathrm{~m}, 2 \mathrm{H}), 3.51(\mathrm{~s}, 3 \mathrm{H}), 3.32(\mathrm{~s}, 3 \mathrm{H}) ;{ }^{13} \mathrm{C}\left\{{ }^{1} \mathrm{H}\right\} \mathrm{NMR}\left(125 \mathrm{MHz}, \mathrm{CD}_{2} \mathrm{Cl}_{2}\right.$, selected resonances) $\delta 197.4\left(\mathrm{~d}, J_{\mathrm{PC}}=\right.$ $4.6 \mathrm{~Hz}), 164.1\left(\mathrm{dd}, J_{\mathrm{PC}}=6.3,126.7 \mathrm{~Hz}\right), 158.0\left(\mathrm{dd}, J_{\mathrm{PC}}=10.4,59.3 \mathrm{~Hz}\right), 146.8,144.2,139.1\left(\mathrm{~d}, J_{\mathrm{PC}}=5.8 \mathrm{~Hz}\right)$, $137.0\left(\mathrm{~d}, J_{\mathrm{PC}}=13.2 \mathrm{~Hz}\right), 135.8,135.6\left(\mathrm{~d}, J_{\mathrm{PC}}=11.5 \mathrm{~Hz}\right), 134.8,134.7,134.0\left(\mathrm{~d}, J_{\mathrm{PC}}=10.4 \mathrm{~Hz}\right), 123.2,112.1(\mathrm{dd}$, $\left.J_{\mathrm{PC}}=1.8,75.0 \mathrm{~Hz}\right), 55.4,55.1 ;{ }^{31} \mathrm{P}\left\{{ }^{1} \mathrm{H}\right\} \mathrm{NMR}\left(162 \mathrm{MHz}, \mathrm{CD}_{2} \mathrm{Cl}_{2}\right) \delta 23.8(\mathrm{~d}, J=43.7 \mathrm{~Hz}), 11.7(\mathrm{~d}, J=43.7 \mathrm{~Hz})$; IR (neat) $v 3053,2833,1655,1597,1569,1460,1436,1417,1288,1263,1206,1153,1095,1050,746,695 \mathrm{~cm}^{-1}$; Anal. Calcd. for $\mathrm{C}_{53} \mathrm{H}_{43} \mathrm{IO}_{3} \mathrm{P}_{2} \mathrm{Pd}: \mathrm{C}, 62.21 ; \mathrm{H}, 4$.24. Found: $\mathrm{C}, 62.40 ; \mathrm{H}, 4.38$; decomposition $>165^{\circ} \mathrm{C}$.

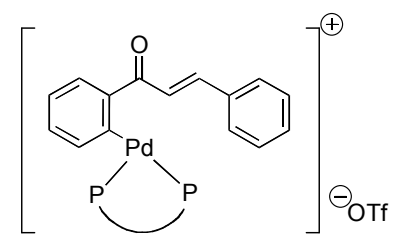

\section{Pd(OTf)(chalcone)(rac-MeOBIPHEP)-complex (I)}

A solution of the PdI(chalcone)(rac-MeOBIPHEP)-complex (206 mg, $0.200 \mathrm{mmol}$ ) and $\operatorname{AgOTf}(51.4 \mathrm{mg}, 0.200 \mathrm{mmol})$ in $\mathrm{CH}_{2} \mathrm{Cl}_{2}(5 \mathrm{~mL})$ was stirred for $20 \mathrm{~min}$ at room temperature. The reaction mixture was filtered over a plug of Celite ${ }^{\circledR}$ and the solvent was removed under reduced pressure to give the desired product as a yellow solid (207 mg, 99\%). ${ }^{1} \mathrm{H}$ NMR (400 $\left.\mathrm{MHz}, \mathrm{CD}_{3} \mathrm{CN}\right) \delta$ 7.85-7.99 (m, 5H), $7.67(\mathrm{~m}, 2 \mathrm{H}), 7.34-7.58(\mathrm{~m}, 18 \mathrm{H}), 7.03-7.11(\mathrm{~m}, 2 \mathrm{H}), 6.94-6.97(\mathrm{~m}, 1 \mathrm{H})$, 6.79-6.85 (m, 2H), $6.68(\mathrm{~d}, 1 \mathrm{H}, J=15.6 \mathrm{~Hz}), 6.49-6.56(\mathrm{~m}, 3 \mathrm{H}), 6.27(\mathrm{~m}, 1 \mathrm{H}), 3.45(\mathrm{~s}, 3 \mathrm{H}), 3.37(\mathrm{~s}, 3 \mathrm{H}) ;{ }^{13} \mathrm{C}\left\{{ }^{1} \mathrm{H}\right\}$ NMR (125 MHz, $\mathrm{CD}_{3} \mathrm{CN}$, selected resonances) $\delta 205.7\left(\mathrm{dd}, J_{\mathrm{PC}}=3.5,8.6 \mathrm{~Hz}\right), 175.2\left(\mathrm{dd}, J_{\mathrm{PC}}=5.8,122.7 \mathrm{~Hz}\right)$, $159.0\left(\mathrm{dd}, J_{\mathrm{PC}}=12.1,73.1 \mathrm{~Hz}\right), 150.9,149.4,114.5\left(\mathrm{~d}, J_{\mathrm{PC}}=2.9 \mathrm{~Hz}\right), 113.9\left(\mathrm{~d}, J_{\mathrm{PC}}=2.3 \mathrm{~Hz}\right), 55.9,55.8 ;{ }^{31} \mathrm{P}\left\{{ }^{1} \mathrm{H}\right\}$ NMR (162 MHz, $\left.\mathrm{CD}_{3} \mathrm{CN}\right) \delta 41.8(\mathrm{~d}, J=46.6 \mathrm{~Hz}), 16.2(\mathrm{~d}, J=46.6 \mathrm{~Hz}) ;{ }^{19} \mathrm{~F} \mathrm{NMR}\left(376 \mathrm{MHz}, \mathrm{CD}_{3} \mathrm{CN}\right) \delta-79.6$; IR (neat) v 3057, 2939, 2837, 1622, 1574, 1557, 1502, 1463, 1438, 1360, 1267, 1153, 1206, 1153, 1101, 1031, $751,694,637 \mathrm{~cm}^{-1}$; decomposition $>145^{\circ} \mathrm{C}$.

\section{$(2 R, 3 S)$-2-ethyl-3-phenyl-indanone (5)}

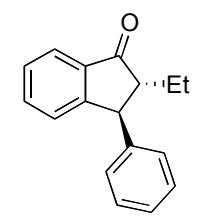

MeLi (1.6M in hexane; $0.75 \mathrm{~mL}, 1.2 \mathrm{mmol})$ was added to a suspension of $\mathrm{CuI}(114 \mathrm{mg}, 0.600$ $\mathrm{mmol})$ in $\mathrm{Et}_{2} \mathrm{O}(4 \mathrm{~mL})$ at $-10{ }^{\circ} \mathrm{C}$. After $15 \mathrm{~min}$ at $-10{ }^{\circ} \mathrm{C}$ a solution of $(\boldsymbol{S})-3 \mathrm{a}(66 \mathrm{mg}, 0.30 \mathrm{mmol}$, $80 \% e e)$ in $\mathrm{Et}_{2} \mathrm{O}(3 \mathrm{~mL})$ was added slowly to the clear solution. The reaction mixture was stirred for $1.5 \mathrm{~h}$ at $-10{ }^{\circ} \mathrm{C}$. The reaction mixture was quenched at $-10{ }^{\circ} \mathrm{C}$ with an aqueous, saturated solution of $\mathrm{NH}_{4} \mathrm{Cl}(5 \mathrm{~mL})$ and warmed up to room temperature. EtOAc $(5 \mathrm{~mL})$ was added and the organic phase was separated, washed with brine and dried over $\mathrm{MgSO}_{4}$. The solvent was removed under reduced pressure and the crude material was dissolved in THF $(2 \mathrm{~mL}) .1 \mathrm{M} \mathrm{HCl}(2 \mathrm{~mL})$ was added and the reaction mixture was stirred at $60{ }^{\circ} \mathrm{C}$ for $12 \mathrm{~h}$. The solution was cooled to room temperature and the organic phase was extracted with EtOAc (2 $\times 5 \mathrm{~mL}$ ). The organic phase was washed with brine and dried over $\mathrm{MgSO}_{4}$. The solvent was removed under reduced pressure and the residue was purified by column chromatography (hexane/ethyl acetate 7:1) to give the 
title compound as a colorless oil [ $55.3 \mathrm{mg}, 78 \%, d r=94: 6$ (the relative configuration was determined to be trans by NOE), $80 \%$ ee (major enantiomer)]. The relative configuration of the major diastereoisomer was determined to be trans by NOE. The enantiomeric excess of the major diastereomer $\mathbf{4}$ was determined by HPLC analysis (Chiracel OD column, $i$-PrOH/hexane $2: 98 ; 1.0 \mathrm{~mL} / \mathrm{min}, 254 \mathrm{~nm}) ;(2 S, 3 R)$ isomer (minor) $t_{\mathrm{R}}=5.8 \mathrm{~min}$ and $(2 R, 3 S)$ isomer (major) $t_{\mathrm{R}}=6.6 \mathrm{~min} ;{ }^{1} \mathrm{H} \mathrm{NMR}\left(400 \mathrm{MHz}, \mathrm{CDCl}_{3}\right) \delta 7.77(\mathrm{~m}, 1 \mathrm{H}), 7.51(\mathrm{~m}, 1 \mathrm{H}), 7.40(\mathrm{M}, 1 \mathrm{H})$, 7.10-7.38 (m, 6H), $4.18(\mathrm{~d}, 1 \mathrm{H}, J=4.3 \mathrm{~Hz}), 2.63(\mathrm{~m}, 1 \mathrm{H}), 1.95(\mathrm{~m}, 1 \mathrm{H}), 1.76(\mathrm{~m}, 1 \mathrm{H}), 0.99$ (t, 3H, J= 7.4 Hz);

${ }^{13} \mathrm{C} \mathrm{NMR}\left(100 \mathrm{MHz}, \mathrm{CDCl}_{3}\right) \delta 208.1,157.0,143.8,136.6,135.2,129.0,128.2,128.0,127.1,126.8,123.6,59.4$, 51.2, 23.7, 11.7; IR (neat) v 3028, 2963, 2933, 2875, 1713, 1604, 1494, 1463, 1321, 1288, 1220, 754, $701 \mathrm{~cm}^{-1}$; Anal. Calcd. for $\mathrm{C}_{17} \mathrm{H}_{16} \mathrm{O}: \mathrm{C}, 86.40 ; \mathrm{H}, 6.82$. Found: $\mathrm{C}, 86.66 ; \mathrm{H}, 6.79$. A copy of the NMR spectra is provided.

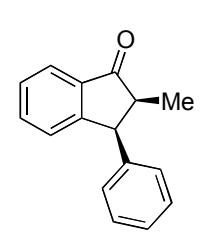

(2S,3S)-2-methyl-3-phenyl-indanone (cis-2m) ${ }^{\text {xxii }}$

A Schlenk tube charged with $5 \mathrm{~mol} \% \mathrm{Pd} / \mathrm{C}(29 \mathrm{mg})$ was evacuated and backfilled with argon two times. After the third evacuation the Schlenk tube was backfilled with $\mathrm{H}_{2}$ and fitted with a balloon filled with $\mathrm{H}_{2}$. A solution of $(\boldsymbol{S})-\mathbf{3 a}(20.0 \mathrm{mg}, 0.091 \mathrm{mmol}, 80 \% e e)$ in EtOAc $(3 \mathrm{~mL})$ was added and the reaction mixture was stirred for $4 \mathrm{~h}$ at room temperature. The catalyst was removed by filtration over a plug of Celite ${ }^{\circledR}$ and the solvent was removed under reduced pressure. The crude reaction mixture was purified by column chromatography on neutralized silica gel (hexane/ethyl acetate 7:1) to give the title compound as a white solid [14.9 mg, 74\%, $d r=93: 7$ (the relative configuration was determined to be cis by NOE), 80\% ee (major enantiomer)]. The enantiomeric excess of the major diastereomer cis-2m was determined by HPLC analysis (Chiracel OJ column, $i$-PrOH/hexane $1: 99 ; 1.0 \mathrm{~mL} / \mathrm{min}, 254 \mathrm{~nm}) ;\left(2 R, 3 R\right.$ ) isomer (minor) $t_{\mathrm{R}}=10.7 \mathrm{~min}$ and $(2 S, 3 S)$ isomer (major) $t_{\mathrm{R}}=13.9 \mathrm{~min} ;{ }^{1} \mathrm{H}$ NMR $\left(400 \mathrm{MHz}, \mathrm{CD}_{2} \mathrm{Cl}_{2}\right) \delta 7.79(\mathrm{~m}, 1 \mathrm{H}), 7.61(\mathrm{~m}, 1 \mathrm{H}), 7.45(\mathrm{~m}, 1 \mathrm{H})$, 7.35 (m, 1H), $7.19-7.28(\mathrm{~m}, 3 \mathrm{H}), 6.88(\mathrm{~m}, 2 \mathrm{H}), 4.76(\mathrm{~d}, 1 \mathrm{H}, J=8.0 \mathrm{~Hz}), 3.07$ (m, 1H), 0.80 (d, 3H, $J=7.5 \mathrm{~Hz})$; ${ }^{13} \mathrm{C}$ NMR $\left(100 \mathrm{MHz}, \mathrm{CD}_{2} \mathrm{Cl}_{2}\right) \delta 208.8,156.7,141.5,137.1,135.5,129.8,128.8,128.5,127.6,127.3,123.7,50.0$, 48.0, 12.8; IR (neat) v3026, 2977, 2932, 2875, 1709, 1604, 1494, 1453, 1374, 1321, 1216, 1091, 962, 755, 723, $701 \mathrm{~cm}^{-1} ; \mathrm{mp} 62-64{ }^{\circ} \mathrm{C}$. A copy of the NMR spectra is provided. 


\section{Crystallographic information for Pd-complex I and ORTEP file (06249)}

Low temperature diffraction data were collected on a Siemens Platform three-circle diffractometer coupled to a Bruker-AXS Smart Apex CCD detector with graphite-monochromated MoK $\alpha$ radiation $(\lambda=0.71073$ $\AA$ ), performing $\phi$ - and $\omega$-scans. The structure was solved by direct methods using SHELXS (Sheldrick, G. M. Acta Cryst. 1990, A46, 467) and refined against $F^{2}$ on all data by full-matrix least squares with SHELXL-97 (Sheldrick, G. M (1997). SHELXL 97, University of Göttingen, Germany). All non-hydrogen atoms were refined anisotropically. All hydrogen atoms were included into the model at geometrically calculated positions and refined using a riding model. The isotropic displacement parameters of all hydrogen atoms were fixed to 1.2 times the $U$ value of the atoms they are linked to (1.5 times for methyl groups). Crystal and structural refinement data for both structures are listed in the following Table 1.

Complex I crystallizes in the monoclinic space group $P 2_{1} / c$ with one molecule of the cationic complex I, one $\mathrm{SO}_{3} \mathrm{CF}_{3}$ counter ion and two solvent molecules $\left(\mathrm{CH}_{2} \mathrm{Cl}_{2}\right)$ in the asymmetric unit. The structure is strongly affected by multiple disorders: Besides a challenging disorder of the 3,5-Xyl-MeOBIPHEP ligand of the main molecule, the $\mathrm{SO}_{3} \mathrm{CF}_{3}$ ion and one of the solvent molecules are highly disordered $\mathrm{CH}_{2} \mathrm{Cl}_{2}$. For the two latter disorders, three components could be resolved, but it appears that more components could be present. All disorders were refined with the help of similarity restraints on 1-2 and 1-3 distances and displacement parameters as well as rigid bond restraints for anisotropic displacement parameters. For the twofold disorder within the main molecule equal isotropic displacement constraints were used as well. The relative occupancies of the disordered components were refined freely, while constraining or restraining the total occupancy of all components to unity. The unusual complexity of the disorders leads to a few spurious clashes between atoms belonging to different components of the various disordered molecules. These clashes are reported by automated structure validation software; however it can be assumed that the random nature of the disorders makes it unlikely for these clashes to occur in the crystal. 


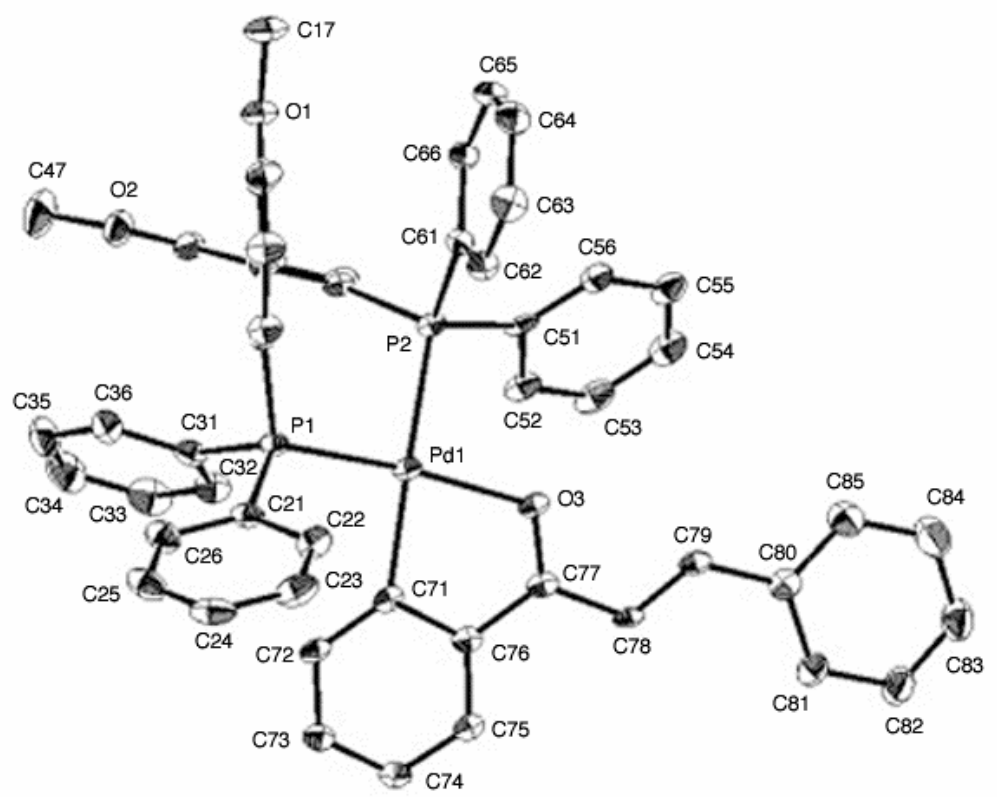


Table 1. Crystal data and structure refinement for 06249.

Identification code

Empirical formula

Formula weight

Temperature

Wavelength

Crystal system

Space group

Unit cell dimensions

Volume

Z

Density (calculated)

Absorption coefficient

$\mathrm{F}(000)$

Crystal size

Theta range for data collection

Index ranges

Reflections collected

Independent reflections

Completeness to theta $=29.13^{\circ}$

Absorption correction

Max. and min. transmission

Refinement method

Data / restraints / parameters

Goodness-of-fit on $\mathrm{F}^{2}$

Final $\mathrm{R}$ indices [I>2sigma(I)]

$\mathrm{R}$ indices (all data)
06249

C56 H47 Cl4 F3 O6 P2 Pd S

1215.14

$100(2) \mathrm{K}$

$0.71073 \AA$

Monoclinic

$\mathrm{P} 2(1) / \mathrm{c}$

$\mathrm{a}=13.1629(5) \AA$

$\mathrm{a}=90^{\circ}$.

$\mathrm{b}=12.1018(4) \AA$

$\mathrm{b}=96.6270(10)^{\circ}$.

$\mathrm{c}=33.8984(12) \AA$

$\mathrm{g}=90^{\circ}$.
4

$1.505 \mathrm{Mg} / \mathrm{m}^{3}$

$0.704 \mathrm{~mm}^{-1}$

2472

$0.32 \times 0.15 \times 0.07 \mathrm{~mm}^{3}$

1.79 to $29.13^{\circ}$.

$-18<=\mathrm{h}<=18,-16<=\mathrm{k}<=16,-46<=1<=46$

114456

$14432[\mathrm{R}($ int $)=0.0669]$

$99.9 \%$

Semi-empirical from equivalents

0.9523 and 0.8060

Full-matrix least-squares on $\mathrm{F}^{2}$

14432 / 2087 / 998

1.061

$\mathrm{R} 1=0.0490, \mathrm{wR} 2=0.1102$

$\mathrm{R} 1=0.0670, \mathrm{wR} 2=0.1193$

Largest diff. peak and hole1.101 and -0.733 e. $\AA^{-3}$ 
Table 2. Atomic coordinates ( $\left.\mathrm{x} 10^{4}\right)$ and equivalent isotropic displacement parameters $\left(\AA^{2} \mathrm{x} 10^{3}\right)$ for 06249 . $U(e q)$ is defined as one third of the trace of the orthogonalized Uij tensor.

\begin{tabular}{|c|c|c|c|c|}
\hline & $\mathrm{x}$ & $\mathrm{y}$ & $\mathrm{z}$ & $\mathrm{U}(\mathrm{eq})$ \\
\hline $\mathrm{P}(1)$ & 1953(1) & $5300(1)$ & $980(1)$ & $17(1)$ \\
\hline $\mathrm{C}(11)$ & $1329(2)$ & $4153(2)$ & 1204(1) & $17(1)$ \\
\hline$C(12)$ & $1880(2)$ & $3188(2)$ & $1325(1)$ & $17(1)$ \\
\hline$C(13)$ & $1342(2)$ & $2289(2)$ & $1462(1)$ & $20(1)$ \\
\hline$C(14)$ & $288(2)$ & $2335(2)$ & $1472(1)$ & $24(1)$ \\
\hline$C(15)$ & $-231(2)$ & $3289(2)$ & $1352(1)$ & $27(1)$ \\
\hline$C(16)$ & $273(2)$ & $4197(2)$ & $1220(1)$ & $23(1)$ \\
\hline $\mathrm{C}(17)$ & $1377(2)$ & $415(2)$ & $1685(1)$ & $32(1)$ \\
\hline $\mathrm{C}(21)$ & $1029(2)$ & $6421(2)$ & 992(1) & $20(1)$ \\
\hline $\mathrm{C}(22)$ & $293(2)$ & $6544(2)$ & $665(1)$ & $26(1)$ \\
\hline$C(23)$ & $-488(2)$ & $7313(3)$ & $672(1)$ & $33(1)$ \\
\hline $\mathrm{C}(24)$ & $-535(2)$ & $7971(2)$ & $1004(1)$ & $33(1)$ \\
\hline$C(25)$ & $188(2)$ & $7852(2)$ & $1329(1)$ & $32(1)$ \\
\hline$C(26)$ & $975(2)$ & $7084(2)$ & $1325(1)$ & $28(1)$ \\
\hline $\mathrm{C}(31)$ & $3079(2)$ & $5644(2)$ & $1318(1)$ & $21(1)$ \\
\hline$C(32)$ & $3998(2)$ & $5828(2)$ & $1158(1)$ & $26(1)$ \\
\hline$C(33)$ & $4877(2)$ & $6108(3)$ & $1408(1)$ & $36(1)$ \\
\hline$C(34)$ & $4841(3)$ & $6205(3)$ & $1809(1)$ & $42(1)$ \\
\hline$C(35)$ & $3936(3)$ & $6015(3)$ & 1971(1) & $38(1)$ \\
\hline$C(36)$ & $3052(2)$ & $5723(2)$ & $1726(1)$ & $29(1)$ \\
\hline$C(41)$ & $3384(2)$ & $2867(2)$ & $938(1)$ & $18(1)$ \\
\hline$C(42)$ & $3004(2)$ & $3054(2)$ & 1302(1) & $18(1)$ \\
\hline$C(43)$ & $3687(2)$ & $3064(2)$ & $1650(1)$ & $23(1)$ \\
\hline$C(44)$ & $4731(2)$ & $2906(2)$ & 1633(1) & $28(1)$ \\
\hline$C(45)$ & $5090(2)$ & $2703(2)$ & $1276(1)$ & $29(1)$ \\
\hline$C(46)$ & $4431(2)$ & $2675(2)$ & $926(1)$ & $24(1)$ \\
\hline$C(47)$ & $3958(3)$ & $3334(3)$ & $2350(1)$ & $45(1)$ \\
\hline $\mathrm{P}(2)$ & 2521(1) & $3035(1)$ & 479(1) & $16(1)$ \\
\hline $\mathrm{C}(51)$ & $3213(19)$ & $2516(17)$ & $77(7)$ & $19(2)$ \\
\hline $\mathrm{C}(52)$ & $4036(16)$ & $3139(17)$ & $-33(6)$ & $25(2)$ \\
\hline$C(53)$ & $4503(13)$ & $2858(17)$ & $-367(5)$ & $30(2)$ \\
\hline
\end{tabular}




\begin{tabular}{|c|c|c|c|c|}
\hline$C(54)$ & $4141(14)$ & $1979(15)$ & $-605(4)$ & $30(2)$ \\
\hline$C(55)$ & $3314(15)$ & $1374(14)$ & $-505(4)$ & $28(2)$ \\
\hline$C(56)$ & $2844(17)$ & $1645(18)$ & $-170(6)$ & $23(2)$ \\
\hline$C(51 \mathrm{~A})$ & $3250(20)$ & $2660(20)$ & $72(9)$ & $22(3)$ \\
\hline$C(52 A)$ & $4064(19)$ & $3350(20)$ & $5(8)$ & $24(2)$ \\
\hline$C(53 \mathrm{~A})$ & $4632(15)$ & $3110(20)$ & $-302(7)$ & $30(3)$ \\
\hline$C(54 \mathrm{~A})$ & $4381(15)$ & $2220(20)$ & $-548(5)$ & $31(3)$ \\
\hline$C(55 \mathrm{~A})$ & $3578(18)$ & $1531(17)$ & $-484(6)$ & $28(3)$ \\
\hline$C(56 \mathrm{~A})$ & $3020(20)$ & $1730(20)$ & $-163(8)$ & $24(2)$ \\
\hline $\mathrm{C}(61)$ & 1497(2) & $2050(2)$ & $496(1)$ & $17(1)$ \\
\hline$C(62)$ & $494(2)$ & $2371(2)$ & $370(1)$ & $21(1)$ \\
\hline$C(63)$ & $-294(2)$ & $1607(2)$ & $364(1)$ & $28(1)$ \\
\hline$C(64)$ & $-89(2)$ & $533(2)$ & $483(1)$ & $29(1)$ \\
\hline$C(65)$ & $902(2)$ & $204(2)$ & $610(1)$ & $25(1)$ \\
\hline$C(66)$ & $1697(2)$ & $957(2)$ & $618(1)$ & $21(1)$ \\
\hline $\mathrm{O}(1)$ & 1919(1) & $1374(2)$ & 1574(1) & $24(1)$ \\
\hline $\mathrm{O}(2)$ & $3263(2)$ & $3241(2)$ & 1991(1) & $28(1)$ \\
\hline $\operatorname{Pd}(1)$ & $2245(1)$ & 4934(1) & $357(1)$ & $16(1)$ \\
\hline $\mathrm{C}(71)$ & $2554(13)$ & $6550(7)$ & $226(3)$ & $15(2)$ \\
\hline$C(72)$ & $2718(13)$ & $7496(8)$ & $461(3)$ & $18(2)$ \\
\hline$C(73)$ & $3069(13)$ & $8477(9)$ & $303(3)$ & $21(2)$ \\
\hline$C(74)$ & $3241(12)$ & $8546(9)$ & $-90(3)$ & $22(2)$ \\
\hline$C(75)$ & $3056(14)$ & $7646(9)$ & $-334(3)$ & $20(1)$ \\
\hline$C(76)$ & $2715(16)$ & $6646(9)$ & $-182(3)$ & $17(1)$ \\
\hline $\mathrm{C}(77)$ & $2530(20)$ & $5654(10)$ & $-421(3)$ & $18(1)$ \\
\hline $\mathrm{C}(78)$ & $2581(13)$ & $5637(7)$ & $-849(3)$ & $18(1)$ \\
\hline $\mathrm{C}(79)$ & $2444(12)$ & $4683(7)$ & $-1051(2)$ & $19(1)$ \\
\hline $\mathrm{C}(80)$ & $2433(6)$ & $4524(6)$ & $-1477(2)$ & $22(1)$ \\
\hline $\mathrm{C}(81)$ & $2620(5)$ & $5391(6)$ & $-1734(2)$ & $26(1)$ \\
\hline $\mathrm{C}(82)$ & $2626(5)$ & $5187(7)$ & $-2135(2)$ & $34(1)$ \\
\hline $\mathrm{C}(83)$ & $2433(6)$ & $4137(8)$ & $-2284(2)$ & $37(2)$ \\
\hline $\mathrm{C}(84)$ & $2235(6)$ & $3282(7)$ & $-2040(2)$ & $40(2)$ \\
\hline $\mathrm{C}(85)$ & $2246(6)$ & $3473(7)$ & $-1637(2)$ & $33(1)$ \\
\hline $\mathrm{O}(3)$ & $2320(40)$ & $4764(13)$ & $-253(3)$ & $20(2)$ \\
\hline$C(71 \mathrm{~A})$ & $2410(16)$ & $6523(8)$ & $162(3)$ & $15(2)$ \\
\hline$C(72 A)$ & $2603(16)$ & $7498(10)$ & $374(3)$ & $18(2)$ \\
\hline
\end{tabular}




\begin{tabular}{|c|c|c|c|c|}
\hline$C(73 A)$ & $2982(15)$ & $8441(11)$ & 197(3) & $21(2)$ \\
\hline$C(74 A)$ & $3166(14)$ & $8418(11)$ & $-199(3)$ & $22(2)$ \\
\hline$C(75 A)$ & $2972(17)$ & $7471(11)$ & $-419(3)$ & $20(1)$ \\
\hline$C(76 A)$ & $2608(19)$ & $6520(10)$ & $-245(3)$ & $17(1)$ \\
\hline $\mathrm{C}(77 \mathrm{~A})$ & $2450(30)$ & $5468(11)$ & $-453(3)$ & $18(1)$ \\
\hline $\mathrm{C}(78 \mathrm{~A})$ & $2512(15)$ & $5345(9)$ & $-881(3)$ & $18(1)$ \\
\hline $\mathrm{C}(79 \mathrm{~A})$ & $2419(14)$ & $4353(8)$ & $-1056(3)$ & $19(1)$ \\
\hline $\mathrm{C}(80 \mathrm{~A})$ & $2411(7)$ & $4079(7)$ & $-1478(2)$ & $22(1)$ \\
\hline $\mathrm{C}(81 \mathrm{~A})$ & $2459(6)$ & $4878(7)$ & $-1771(2)$ & $29(1)$ \\
\hline $\mathrm{C}(82 \mathrm{~A})$ & $2423(8)$ & $4572(9)$ & $-2169(2)$ & $37(2)$ \\
\hline$C(83 A)$ & $2342(7)$ & $3464(8)$ & $-2269(2)$ & $38(2)$ \\
\hline $\mathrm{C}(84 \mathrm{~A})$ & $2306(6)$ & $2664(8)$ & $-1981(2)$ & $34(2)$ \\
\hline$C(85 A)$ & $2335(6)$ & $2970(7)$ & $-1588(2)$ & $27(1)$ \\
\hline $\mathrm{O}(3 \mathrm{~A})$ & $2230(40)$ & $4643(14)$ & $-254(4)$ & $20(2)$ \\
\hline $\mathrm{Cl}(1)$ & $4207(1)$ & $10109(1)$ & $2063(1)$ & $56(1)$ \\
\hline $\mathrm{C}(1 \mathrm{~S})$ & $4178(3)$ & $10140(3)$ & $1541(1)$ & $38(1)$ \\
\hline $\mathrm{Cl}(2)$ & $3690(1)$ & $8890(1)$ & $1324(1)$ & $65(1)$ \\
\hline $\mathrm{Cl}(3)$ & $10024(3)$ & $3781(5)$ & $2542(1)$ & $89(2)$ \\
\hline$C(2 S)$ & $9097(7)$ & $2806(10)$ & $2354(3)$ & $86(3)$ \\
\hline $\mathrm{Cl}(4)$ & $8053(2)$ & $2769(2)$ & $2640(1)$ & $65(1)$ \\
\hline $\mathrm{Cl}(3 \mathrm{~A})$ & $9814(11)$ & $3349(7)$ & 2554(4) & 191(5) \\
\hline $\mathrm{C}(2 \mathrm{SA})$ & $10697(12)$ & $4286(13)$ & 2391(8) & $121(6)$ \\
\hline $\mathrm{Cl}(4 \mathrm{~A})$ & $10129(2)$ & $5453(2)$ & $2142(1)$ & $49(1)$ \\
\hline $\mathrm{Cl}(3 \mathrm{~B})$ & $9900(14)$ & $2250(13)$ & $2553(5)$ & $205(7)$ \\
\hline $\mathrm{C}(1 \mathrm{SB})$ & $9770(20)$ & $3652(16)$ & $2406(11)$ & $124(6)$ \\
\hline $\mathrm{Cl}(4 \mathrm{~B})$ & $10910(20)$ & $4170(20)$ & $2244(6)$ & $268(11)$ \\
\hline $\mathrm{S}(1)$ & $7597(2)$ & $711(2)$ & $1498(1)$ & $28(1)$ \\
\hline $\mathrm{O}(4)$ & $7602(5)$ & $1772(5)$ & $1315(2)$ & $30(1)$ \\
\hline $\mathrm{O}(5)$ & $8520(4)$ & $90(4)$ & $1500(2)$ & $50(1)$ \\
\hline $\mathrm{O}(6)$ & $6661(4)$ & $85(4)$ & $1432(2)$ & $52(1)$ \\
\hline $\mathrm{C}(1)$ & $7603(5)$ & $1082(5)$ & $2021(2)$ & $46(1)$ \\
\hline $\mathrm{F}(1)$ & $7564(6)$ & $221(5)$ & $2255(2)$ & $78(2)$ \\
\hline $\mathrm{F}(2)$ & $6805(4)$ & $1724(5)$ & $2074(2)$ & $66(1)$ \\
\hline $\mathrm{F}(3)$ & $8448(4)$ & $1650(5)$ & $2144(2)$ & $81(2)$ \\
\hline $\mathrm{S}(1 \mathrm{~A})$ & $7538(4)$ & $869(4)$ & $1362(1)$ & $39(1)$ \\
\hline $\mathrm{O}(4 \mathrm{~A})$ & $6550(7)$ & $612(11)$ & $1135(4)$ & $55(3)$ \\
\hline
\end{tabular}




\begin{tabular}{lllll}
$\mathrm{O}(5 \mathrm{~A})$ & $7664(9)$ & $1999(6)$ & $1461(3)$ & $32(2)$ \\
$\mathrm{O}(6 \mathrm{~A})$ & $8355(8)$ & $321(8)$ & $1223(4)$ & $79(3)$ \\
$\mathrm{C}(1 \mathrm{~A})$ & $7361(8)$ & $212(8)$ & $1829(3)$ & $70(2)$ \\
$\mathrm{F}(1 \mathrm{~A})$ & $8185(10)$ & $303(11)$ & $2092(4)$ & $144(4)$ \\
$\mathrm{F}(2 \mathrm{~A})$ & $7141(9)$ & $-866(6)$ & $1751(3)$ & $123(4)$ \\
$\mathrm{F}(3 \mathrm{~A})$ & $6564(8)$ & $622(8)$ & $1975(3)$ & $86(3)$ \\
$\mathrm{S}(1 \mathrm{~B})$ & $7521(4)$ & $1215(4)$ & $1755(2)$ & $28(1)$ \\
$\mathrm{O}(4 \mathrm{~B})$ & $6675(11)$ & $1020(14)$ & $1966(5)$ & $35(3)$ \\
$\mathrm{O}(5 \mathrm{~B})$ & $7534(12)$ & $2270(9)$ & $1564(5)$ & $25(3)$ \\
$\mathrm{O}(6 \mathrm{~B})$ & $8511(8)$ & $820(12)$ & $1919(5)$ & $51(4)$ \\
$\mathrm{C}(1 \mathrm{~B})$ & $7266(10)$ & $308(11)$ & $1323(4)$ & $34(3)$ \\
$\mathrm{F}(1 \mathrm{~B})$ & $6344(10)$ & $512(18)$ & $1134(7)$ & $52(5)$ \\
$\mathrm{F}(2 \mathrm{~B})$ & $7239(12)$ & $-759(10)$ & $1441(4)$ & $49(3)$ \\
$\mathrm{F}(3 \mathrm{~B})$ & $7975(9)$ & $361(14)$ & $1064(4)$ & $35(3)$ \\
\hline
\end{tabular}

Table 3. Bond lengths $[\AA]$ and angles $\left[{ }^{\circ}\right]$ for 06249.

\begin{tabular}{ll}
\hline $\mathrm{P}(1)-\mathrm{C}(31)$ & $1.812(3)$ \\
$\mathrm{P}(1)-\mathrm{C}(11)$ & $1.823(3)$ \\
$\mathrm{P}(1)-\mathrm{C}(21)$ & $1.825(3)$ \\
$\mathrm{P}(1)-\mathrm{Pd}(1)$ & $2.2357(7)$ \\
$\mathrm{C}(11)-\mathrm{C}(16)$ & $1.398(3)$ \\
$\mathrm{C}(11)-\mathrm{C}(12)$ & $1.411(3)$ \\
$\mathrm{C}(12)-\mathrm{C}(13)$ & $1.406(3)$ \\
$\mathrm{C}(12)-\mathrm{C}(42)$ & $1.500(3)$ \\
$\mathrm{C}(13)-\mathrm{O}(1)$ & $1.371(3)$ \\
$\mathrm{C}(13)-\mathrm{C}(14)$ & $1.393(4)$ \\
$\mathrm{C}(14)-\mathrm{C}(15)$ & $1.380(4)$ \\
$\mathrm{C}(15)-\mathrm{C}(16)$ & $1.384(4)$ \\
$\mathrm{C}(17)-\mathrm{O}(1)$ & $1.435(3)$ \\
$\mathrm{C}(21)-\mathrm{C}(26)$ & $1.393(4)$ \\
$\mathrm{C}(21)-\mathrm{C}(22)$ & $1.395(4)$ \\
$\mathrm{C}(22)-\mathrm{C}(23)$ & $1.390(4)$ \\
$\mathrm{C}(23)-\mathrm{C}(24)$ & $1.387(5)$ \\
$\mathrm{C}(24)-\mathrm{C}(25)$ & $1.378(5)$ \\
$\mathrm{C}(25)-\mathrm{C}(26)$ & $1.392(4)$ \\
$\mathrm{C}(31)-\mathrm{C}(36)$ & $1.391(4)$ \\
& \\
&
\end{tabular}


S36

\begin{tabular}{|c|c|}
\hline$C(31)-C(32)$ & $1.399(4)$ \\
\hline$C(32)-C(33)$ & $1.393(4)$ \\
\hline$C(33)-C(34)$ & $1.370(5)$ \\
\hline$C(34)-C(35)$ & $1.388(5)$ \\
\hline$C(35)-C(36)$ & $1.395(4)$ \\
\hline$C(41)-C(46)$ & $1.402(3)$ \\
\hline$C(41)-C(42)$ & $1.403(4)$ \\
\hline $\mathrm{C}(41)-\mathrm{P}(2)$ & $1.828(3)$ \\
\hline$C(42)-C(43)$ & $1.400(3)$ \\
\hline $\mathrm{C}(43)-\mathrm{O}(2)$ & $1.357(3)$ \\
\hline$C(43)-C(44)$ & $1.396(4)$ \\
\hline$C(44)-C(45)$ & $1.371(4)$ \\
\hline$C(45)-C(46)$ & $1.388(4)$ \\
\hline $\mathrm{C}(47)-\mathrm{O}(2)$ & $1.439(4)$ \\
\hline $\mathrm{P}(2)-\mathrm{C}(61)$ & $1.805(3)$ \\
\hline $\mathrm{P}(2)-\mathrm{C}(51 \mathrm{~A})$ & $1.823(10)$ \\
\hline $\mathrm{P}(2)-\mathrm{C}(51)$ & $1.836(8)$ \\
\hline $\mathrm{P}(2)-\operatorname{Pd}(1)$ & $2.3562(7)$ \\
\hline$C(51)-C(56)$ & $1.397(9)$ \\
\hline$C(51)-C(52)$ & $1.405(8)$ \\
\hline$C(52)-C(53)$ & $1.391(9)$ \\
\hline$C(53)-C(54)$ & $1.385(9)$ \\
\hline$C(54)-C(55)$ & $1.386(8)$ \\
\hline$C(55)-C(56)$ & $1.395(9)$ \\
\hline$C(51 A)-C(56 A)$ & $1.392(10)$ \\
\hline $\mathrm{C}(51 \mathrm{~A})-\mathrm{C}(52 \mathrm{~A})$ & $1.398(10)$ \\
\hline $\mathrm{C}(52 \mathrm{~A})-\mathrm{C}(53 \mathrm{~A})$ & $1.379(10)$ \\
\hline $\mathrm{C}(53 \mathrm{~A})-\mathrm{C}(54 \mathrm{~A})$ & $1.380(10)$ \\
\hline $\mathrm{C}(54 \mathrm{~A})-\mathrm{C}(55 \mathrm{~A})$ & $1.383(10)$ \\
\hline$C(55 \mathrm{~A})-\mathrm{C}(56 \mathrm{~A})$ & $1.400(10)$ \\
\hline $\mathrm{C}(61)-\mathrm{C}(62)$ & $1.395(3)$ \\
\hline $\mathrm{C}(61)-\mathrm{C}(66)$ & $1.402(3)$ \\
\hline $\mathrm{C}(62)-\mathrm{C}(63)$ & $1.389(4)$ \\
\hline$C(63)-C(64)$ & $1.378(4)$ \\
\hline $\mathrm{C}(64)-\mathrm{C}(65)$ & $1.384(4)$ \\
\hline$C(65)-C(66)$ & $1.386(4)$ \\
\hline
\end{tabular}




\begin{tabular}{|c|c|}
\hline $\operatorname{Pd}(1)-C(71 A)$ & $2.053(9)$ \\
\hline $\operatorname{Pd}(1)-C(71)$ & $2.056(8)$ \\
\hline $\operatorname{Pd}(1)-\mathrm{O}(3)$ & $2.089(8)$ \\
\hline $\operatorname{Pd}(1)-O(3 A)$ & $2.098(10)$ \\
\hline$C(71)-C(72)$ & $1.396(8)$ \\
\hline$C(71)-C(76)$ & $1.430(8)$ \\
\hline$C(72)-C(73)$ & $1.402(9)$ \\
\hline$C(73)-C(74)$ & $1.378(8)$ \\
\hline$C(74)-C(75)$ & $1.373(7)$ \\
\hline$C(75)-C(76)$ & $1.410(8)$ \\
\hline $\mathrm{C}(76)-\mathrm{C}(77)$ & $1.453(7)$ \\
\hline $\mathrm{C}(77)-\mathrm{O}(3)$ & $1.264(8)$ \\
\hline $\mathrm{C}(77)-\mathrm{C}(78)$ & $1.459(8)$ \\
\hline $\mathrm{C}(78)-\mathrm{C}(79)$ & $1.344(7)$ \\
\hline $\mathrm{C}(79)-\mathrm{C}(80)$ & $1.455(8)$ \\
\hline $\mathrm{C}(80)-\mathrm{C}(85)$ & $1.394(8)$ \\
\hline $\mathrm{C}(80)-\mathrm{C}(81)$ & $1.405(7)$ \\
\hline $\mathrm{C}(81)-\mathrm{C}(82)$ & $1.381(7)$ \\
\hline $\mathrm{C}(82)-\mathrm{C}(83)$ & $1.381(8)$ \\
\hline C(83)-C(84) & $1.369(9)$ \\
\hline$C(84)-C(85)$ & $1.385(8)$ \\
\hline$C(71 \mathrm{~A})-\mathrm{C}(72 \mathrm{~A})$ & $1.390(9)$ \\
\hline$C(71 A)-C(76 A)$ & $1.430(9)$ \\
\hline$C(72 A)-C(73 A)$ & $1.407(10)$ \\
\hline$C(73 A)-C(74 A)$ & $1.390(9)$ \\
\hline$C(74 \mathrm{~A})-\mathrm{C}(75 \mathrm{~A})$ & $1.375(8)$ \\
\hline$C(75 A)-C(76 A)$ & $1.403(9)$ \\
\hline$C(76 A)-C(77 A)$ & $1.460(8)$ \\
\hline $\mathrm{C}(77 \mathrm{~A})-\mathrm{O}(3 \mathrm{~A})$ & $1.259(9)$ \\
\hline$C(77 A)-C(78 A)$ & $1.468(9)$ \\
\hline $\mathrm{C}(78 \mathrm{~A})-\mathrm{C}(79 \mathrm{~A})$ & $1.339(8)$ \\
\hline$C(79 A)-C(80 A)$ & $1.468(9)$ \\
\hline$C(80 A)-C(81 A)$ & $1.391(8)$ \\
\hline $\mathrm{C}(80 \mathrm{~A})-\mathrm{C}(85 \mathrm{~A})$ & $1.394(8)$ \\
\hline $\mathrm{C}(81 \mathrm{~A})-\mathrm{C}(82 \mathrm{~A})$ & $1.397(9)$ \\
\hline $\mathrm{C}(82 \mathrm{~A})-\mathrm{C}(83 \mathrm{~A})$ & $1.383(10)$ \\
\hline
\end{tabular}


S38

\begin{tabular}{|c|c|}
\hline$C(83 A)-C(84 A)$ & $1.379(9)$ \\
\hline $\mathrm{C}(84 \mathrm{~A})-\mathrm{C}(85 \mathrm{~A})$ & $1.380(8)$ \\
\hline $\mathrm{Cl}(1)-\mathrm{C}(1 \mathrm{~S})$ & $1.765(3)$ \\
\hline $\mathrm{C}(1 \mathrm{~S})-\mathrm{Cl}(2)$ & $1.770(3)$ \\
\hline $\mathrm{Cl}(3)-\mathrm{C}(2 \mathrm{~S})$ & $1.763(8)$ \\
\hline $\mathrm{C}(2 \mathrm{~S})-\mathrm{Cl}(4)$ & $1.772(7)$ \\
\hline $\mathrm{Cl}(3 \mathrm{~A})-\mathrm{C}(2 \mathrm{SA})$ & $1.758(9)$ \\
\hline $\mathrm{C}(2 \mathrm{SA})-\mathrm{Cl}(4 \mathrm{~A})$ & $1.766(8)$ \\
\hline $\mathrm{Cl}(3 \mathrm{~B})-\mathrm{C}(1 \mathrm{SB})$ & $1.771(9)$ \\
\hline $\mathrm{C}(1 \mathrm{SB})-\mathrm{Cl}(4 \mathrm{~B})$ & $1.773(9)$ \\
\hline $\mathrm{S}(1)-\mathrm{O}(4)$ & $1.426(5)$ \\
\hline $\mathrm{S}(1)-\mathrm{O}(5)$ & $1.428(5)$ \\
\hline $\mathrm{S}(1)-\mathrm{O}(6)$ & $1.441(5)$ \\
\hline$S(1)-C(1)$ & $1.829(8)$ \\
\hline$C(1)-F(1)$ & $1.315(8)$ \\
\hline$C(1)-F(3)$ & $1.333(8)$ \\
\hline $\mathrm{C}(1)-\mathrm{F}(2)$ & $1.336(8)$ \\
\hline $\mathrm{S}(1 \mathrm{~A})-\mathrm{O}(6 \mathrm{~A})$ & $1.391(8)$ \\
\hline $\mathrm{S}(1 \mathrm{~A})-\mathrm{O}(5 \mathrm{~A})$ & $1.413(8)$ \\
\hline $\mathrm{S}(1 \mathrm{~A})-\mathrm{O}(4 \mathrm{~A})$ & $1.465(9)$ \\
\hline$S(1 A)-C(1 A)$ & $1.811(10)$ \\
\hline$C(1 A)-F(3 A)$ & $1.309(11)$ \\
\hline$C(1 \mathrm{~A})-\mathrm{F}(1 \mathrm{~A})$ & $1.327(11)$ \\
\hline$C(1 \mathrm{~A})-\mathrm{F}(2 \mathrm{~A})$ & $1.356(10)$ \\
\hline $\mathrm{S}(1 \mathrm{~B})-\mathrm{O}(4 \mathrm{~B})$ & $1.413(10)$ \\
\hline $\mathrm{S}(1 \mathrm{~B})-\mathrm{O}(5 \mathrm{~B})$ & $1.432(10)$ \\
\hline $\mathrm{S}(1 \mathrm{~B})-\mathrm{O}(6 \mathrm{~B})$ & $1.438(10)$ \\
\hline$S(1 B)-C(1 B)$ & $1.831(13)$ \\
\hline$C(1 B)-F(1 B)$ & $1.328(12)$ \\
\hline$C(1 B)-F(3 B)$ & $1.354(12)$ \\
\hline $\mathrm{C}(1 \mathrm{~B})-\mathrm{F}(2 \mathrm{~B})$ & $1.354(12)$ \\
\hline $\mathrm{C}(31)-\mathrm{P}(1)-\mathrm{C}(11)$ & $106.71(12)$ \\
\hline$C(31)-P(1)-C(21)$ & $108.26(12)$ \\
\hline $\mathrm{C}(11)-\mathrm{P}(1)-\mathrm{C}(21)$ & $102.88(11)$ \\
\hline $\mathrm{C}(31)-\mathrm{P}(1)-\mathrm{Pd}(1)$ & $115.16(9)$ \\
\hline $\mathrm{C}(11)-\mathrm{P}(1)-\mathrm{Pd}(1)$ & $112.19(8)$ \\
\hline
\end{tabular}




\begin{tabular}{|c|c|}
\hline$C(21)-P(1)-P d(1)$ & $110.83(9)$ \\
\hline$C(16)-C(11)-C(12)$ & $120.0(2)$ \\
\hline$C(16)-C(11)-P(1)$ & $119.02(19)$ \\
\hline $\mathrm{C}(12)-\mathrm{C}(11)-\mathrm{P}(1)$ & $120.67(18)$ \\
\hline $\mathrm{C}(13)-\mathrm{C}(12)-\mathrm{C}(11)$ & $118.4(2)$ \\
\hline $\mathrm{C}(13)-\mathrm{C}(12)-\mathrm{C}(42)$ & $118.1(2)$ \\
\hline $\mathrm{C}(11)-\mathrm{C}(12)-\mathrm{C}(42)$ & $123.4(2)$ \\
\hline $\mathrm{O}(1)-\mathrm{C}(13)-\mathrm{C}(14)$ & $123.2(2)$ \\
\hline $\mathrm{O}(1)-\mathrm{C}(13)-\mathrm{C}(12)$ & $115.6(2)$ \\
\hline $\mathrm{C}(14)-\mathrm{C}(13)-\mathrm{C}(12)$ & $121.2(2)$ \\
\hline$C(15)-C(14)-C(13)$ & $119.2(2)$ \\
\hline$C(14)-C(15)-C(16)$ & $121.4(2)$ \\
\hline $\mathrm{C}(15)-\mathrm{C}(16)-\mathrm{C}(11)$ & $119.8(2)$ \\
\hline$C(26)-C(21)-C(22)$ & 119.2(3) \\
\hline $\mathrm{C}(26)-\mathrm{C}(21)-\mathrm{P}(1)$ & $122.9(2)$ \\
\hline $\mathrm{C}(22)-\mathrm{C}(21)-\mathrm{P}(1)$ & $117.7(2)$ \\
\hline $\mathrm{C}(23)-\mathrm{C}(22)-\mathrm{C}(21)$ & $120.3(3)$ \\
\hline $\mathrm{C}(24)-\mathrm{C}(23)-\mathrm{C}(22)$ & $120.1(3)$ \\
\hline $\mathrm{C}(25)-\mathrm{C}(24)-\mathrm{C}(23)$ & $119.8(3)$ \\
\hline $\mathrm{C}(24)-\mathrm{C}(25)-\mathrm{C}(26)$ & $120.5(3)$ \\
\hline $\mathrm{C}(25)-\mathrm{C}(26)-\mathrm{C}(21)$ & $120.0(3)$ \\
\hline $\mathrm{C}(36)-\mathrm{C}(31)-\mathrm{C}(32)$ & $119.7(3)$ \\
\hline $\mathrm{C}(36)-\mathrm{C}(31)-\mathrm{P}(1)$ & $122.1(2)$ \\
\hline $\mathrm{C}(32)-\mathrm{C}(31)-\mathrm{P}(1)$ & $118.2(2)$ \\
\hline $\mathrm{C}(33)-\mathrm{C}(32)-\mathrm{C}(31)$ & $119.8(3)$ \\
\hline $\mathrm{C}(34)-\mathrm{C}(33)-\mathrm{C}(32)$ & $120.2(3)$ \\
\hline $\mathrm{C}(33)-\mathrm{C}(34)-\mathrm{C}(35)$ & $120.5(3)$ \\
\hline $\mathrm{C}(34)-\mathrm{C}(35)-\mathrm{C}(36)$ & $120.0(3)$ \\
\hline $\mathrm{C}(31)-\mathrm{C}(36)-\mathrm{C}(35)$ & $119.7(3)$ \\
\hline$C(46)-C(41)-C(42)$ & $120.2(2)$ \\
\hline $\mathrm{C}(46)-\mathrm{C}(41)-\mathrm{P}(2)$ & $120.8(2)$ \\
\hline $\mathrm{C}(42)-\mathrm{C}(41)-\mathrm{P}(2)$ & $118.65(18)$ \\
\hline$C(43)-C(42)-C(41)$ & $118.9(2)$ \\
\hline$C(43)-C(42)-C(12)$ & $119.7(2)$ \\
\hline $\mathrm{C}(41)-\mathrm{C}(42)-\mathrm{C}(12)$ & $121.3(2)$ \\
\hline $\mathrm{O}(2)-\mathrm{C}(43)-\mathrm{C}(44)$ & $124.0(2)$ \\
\hline
\end{tabular}




\begin{tabular}{|c|c|}
\hline $\mathrm{O}(2)-\mathrm{C}(43)-\mathrm{C}(42)$ & $115.7(2)$ \\
\hline$C(44)-C(43)-C(42)$ & $120.3(3)$ \\
\hline$C(45)-C(44)-C(43)$ & $120.1(3)$ \\
\hline $\mathrm{C}(44)-\mathrm{C}(45)-\mathrm{C}(46)$ & $121.0(3)$ \\
\hline $\mathrm{C}(45)-\mathrm{C}(46)-\mathrm{C}(41)$ & $119.4(3)$ \\
\hline$C(61)-P(2)-C(51 A)$ & $108.6(7)$ \\
\hline $\mathrm{C}(61)-\mathrm{P}(2)-\mathrm{C}(41)$ & $106.99(11)$ \\
\hline $\mathrm{C}(51 \mathrm{~A})-\mathrm{P}(2)-\mathrm{C}(41)$ & $106.7(14)$ \\
\hline $\mathrm{C}(61)-\mathrm{P}(2)-\mathrm{C}(51)$ & $103.6(5)$ \\
\hline $\mathrm{C}(51 \mathrm{~A})-\mathrm{P}(2)-\mathrm{C}(51)$ & $5.8(13)$ \\
\hline $\mathrm{C}(41)-\mathrm{P}(2)-\mathrm{C}(51)$ & $106.2(10)$ \\
\hline $\mathrm{C}(61)-\mathrm{P}(2)-\mathrm{Pd}(1)$ & $123.34(8)$ \\
\hline $\mathrm{C}(51 \mathrm{~A})-\mathrm{P}(2)-\mathrm{Pd}(1)$ & $101.0(11)$ \\
\hline $\mathrm{C}(41)-\mathrm{P}(2)-\mathrm{Pd}(1)$ & $109.06(8)$ \\
\hline $\mathrm{C}(51)-\mathrm{P}(2)-\mathrm{Pd}(1)$ & $106.4(8)$ \\
\hline $\mathrm{C}(56)-\mathrm{C}(51)-\mathrm{C}(52)$ & $118.1(8)$ \\
\hline $\mathrm{C}(56)-\mathrm{C}(51)-\mathrm{P}(2)$ & $122.3(10)$ \\
\hline $\mathrm{C}(52)-\mathrm{C}(51)-\mathrm{P}(2)$ & $118.5(8)$ \\
\hline $\mathrm{C}(53)-\mathrm{C}(52)-\mathrm{C}(51)$ & $120.7(8)$ \\
\hline $\mathrm{C}(54)-\mathrm{C}(53)-\mathrm{C}(52)$ & $120.5(8)$ \\
\hline $\mathrm{C}(53)-\mathrm{C}(54)-\mathrm{C}(55)$ & $119.4(7)$ \\
\hline$C(54)-C(55)-C(56)$ & $120.6(8)$ \\
\hline$C(55)-C(56)-C(51)$ & $120.6(8)$ \\
\hline$C(56 A)-C(51 A)-C(52 A)$ & $120.2(9)$ \\
\hline $\mathrm{C}(56 \mathrm{~A})-\mathrm{C}(51 \mathrm{~A})-\mathrm{P}(2)$ & $122.6(11)$ \\
\hline $\mathrm{C}(52 \mathrm{~A})-\mathrm{C}(51 \mathrm{~A})-\mathrm{P}(2)$ & $117.2(11)$ \\
\hline$C(53 \mathrm{~A})-\mathrm{C}(52 \mathrm{~A})-\mathrm{C}(51 \mathrm{~A})$ & $119.6(10)$ \\
\hline$C(52 A)-C(53 A)-C(54 A)$ & $120.3(9)$ \\
\hline $\mathrm{C}(53 \mathrm{~A})-\mathrm{C}(54 \mathrm{~A})-\mathrm{C}(55 \mathrm{~A})$ & $120.7(9)$ \\
\hline$C(54 A)-C(55 A)-C(56 A)$ & $119.7(10)$ \\
\hline$C(51 A)-C(56 A)-C(55 A)$ & $119.3(10)$ \\
\hline$C(62)-C(61)-C(66)$ & $119.5(2)$ \\
\hline $\mathrm{C}(62)-\mathrm{C}(61)-\mathrm{P}(2)$ & 119.31(19) \\
\hline $\mathrm{C}(66)-\mathrm{C}(61)-\mathrm{P}(2)$ & $121.14(19)$ \\
\hline $\mathrm{C}(63)-\mathrm{C}(62)-\mathrm{C}(61)$ & $119.9(2)$ \\
\hline$C(64)-C(63)-C(62)$ & $120.2(3)$ \\
\hline
\end{tabular}




\begin{tabular}{|c|c|}
\hline$C(63)-C(64)-C(65)$ & $120.5(3)$ \\
\hline$C(64)-C(65)-C(66)$ & $120.0(3)$ \\
\hline$C(65)-C(66)-C(61)$ & $119.9(2)$ \\
\hline $\mathrm{C}(13)-\mathrm{O}(1)-\mathrm{C}(17)$ & $116.7(2)$ \\
\hline $\mathrm{C}(43)-\mathrm{O}(2)-\mathrm{C}(47)$ & $116.7(2)$ \\
\hline$C(71 \mathrm{~A})-\mathrm{Pd}(1)-\mathrm{C}(71)$ & $7.7(8)$ \\
\hline $\mathrm{C}(71 \mathrm{~A})-\mathrm{Pd}(1)-\mathrm{O}(3)$ & $75.8(5)$ \\
\hline $\mathrm{C}(71)-\mathrm{Pd}(1)-\mathrm{O}(3)$ & $81.3(4)$ \\
\hline $\mathrm{C}(71 \mathrm{~A})-\mathrm{Pd}(1)-\mathrm{O}(3 \mathrm{~A})$ & $80.1(5)$ \\
\hline $\mathrm{C}(71)-\mathrm{Pd}(1)-\mathrm{O}(3 \mathrm{~A})$ & $85.8(4)$ \\
\hline $\mathrm{O}(3)-\mathrm{Pd}(1)-\mathrm{O}(3 \mathrm{~A})$ & $5.2(14)$ \\
\hline $\mathrm{C}(71 \mathrm{~A})-\mathrm{Pd}(1)-\mathrm{P}(1)$ & $98.9(3)$ \\
\hline $\mathrm{C}(71)-\mathrm{Pd}(1)-\mathrm{P}(1)$ & $94.3(3)$ \\
\hline $\mathrm{O}(3)-\mathrm{Pd}(1)-\mathrm{P}(1)$ & $170.7(12)$ \\
\hline $\mathrm{O}(3 \mathrm{~A})-\mathrm{Pd}(1)-\mathrm{P}(1)$ & $169.4(15)$ \\
\hline $\mathrm{C}(71 \mathrm{~A})-\mathrm{Pd}(1)-\mathrm{P}(2)$ & $161.9(5)$ \\
\hline $\mathrm{C}(71)-\mathrm{Pd}(1)-\mathrm{P}(2)$ & $159.3(5)$ \\
\hline $\mathrm{O}(3)-\mathrm{Pd}(1)-\mathrm{P}(2)$ & $93.1(6)$ \\
\hline $\mathrm{O}(3 \mathrm{~A})-\mathrm{Pd}(1)-\mathrm{P}(2)$ & $89.7(7)$ \\
\hline $\mathrm{P}(1)-\mathrm{Pd}(1)-\mathrm{P}(2)$ & $93.79(2)$ \\
\hline $\mathrm{C}(72)-\mathrm{C}(71)-\mathrm{C}(76)$ & 117.1(7) \\
\hline $\mathrm{C}(72)-\mathrm{C}(71)-\mathrm{Pd}(1)$ & $132.7(6)$ \\
\hline $\mathrm{C}(76)-\mathrm{C}(71)-\mathrm{Pd}(1)$ & $109.9(5)$ \\
\hline $\mathrm{C}(71)-\mathrm{C}(72)-\mathrm{C}(73)$ & $120.8(7)$ \\
\hline $\mathrm{C}(74)-\mathrm{C}(73)-\mathrm{C}(72)$ & $121.2(7)$ \\
\hline $\mathrm{C}(75)-\mathrm{C}(74)-\mathrm{C}(73)$ & $119.9(7)$ \\
\hline $\mathrm{C}(74)-\mathrm{C}(75)-\mathrm{C}(76)$ & $120.1(7)$ \\
\hline $\mathrm{C}(75)-\mathrm{C}(76)-\mathrm{C}(71)$ & $120.8(6)$ \\
\hline $\mathrm{C}(75)-\mathrm{C}(76)-\mathrm{C}(77)$ & $123.1(7)$ \\
\hline $\mathrm{C}(71)-\mathrm{C}(76)-\mathrm{C}(77)$ & $116.0(6)$ \\
\hline $\mathrm{O}(3)-\mathrm{C}(77)-\mathrm{C}(76)$ & $118.9(7)$ \\
\hline $\mathrm{O}(3)-\mathrm{C}(77)-\mathrm{C}(78)$ & $118.4(7)$ \\
\hline $\mathrm{C}(76)-\mathrm{C}(77)-\mathrm{C}(78)$ & $122.7(7)$ \\
\hline $\mathrm{C}(79)-\mathrm{C}(78)-\mathrm{C}(77)$ & $119.9(7)$ \\
\hline $\mathrm{C}(78)-\mathrm{C}(79)-\mathrm{C}(80)$ & $127.2(7)$ \\
\hline $\mathrm{C}(85)-\mathrm{C}(80)-\mathrm{C}(81)$ & $118.5(6)$ \\
\hline
\end{tabular}




\begin{tabular}{|c|c|}
\hline $\mathrm{C}(85)-\mathrm{C}(80)-\mathrm{C}(79)$ & $119.2(5)$ \\
\hline $\mathrm{C}(81)-\mathrm{C}(80)-\mathrm{C}(79)$ & $122.3(5)$ \\
\hline $\mathrm{C}(82)-\mathrm{C}(81)-\mathrm{C}(80)$ & $119.9(6)$ \\
\hline $\mathrm{C}(83)-\mathrm{C}(82)-\mathrm{C}(81)$ & $120.2(6)$ \\
\hline $\mathrm{C}(84)-\mathrm{C}(83)-\mathrm{C}(82)$ & $120.9(6)$ \\
\hline$C(83)-C(84)-C(85)$ & $119.3(7)$ \\
\hline $\mathrm{C}(84)-\mathrm{C}(85)-\mathrm{C}(80)$ & $121.2(6)$ \\
\hline $\mathrm{C}(77)-\mathrm{O}(3)-\mathrm{Pd}(1)$ & $113.6(6)$ \\
\hline$C(72 A)-C(71 A)-C(76 A)$ & $117.2(8)$ \\
\hline$C(72 A)-C(71 A)-P d(1)$ & $130.4(7)$ \\
\hline$C(76 \mathrm{~A})-\mathrm{C}(71 \mathrm{~A})-\mathrm{Pd}(1)$ & $110.2(7)$ \\
\hline$C(71 A)-C(72 A)-C(73 A)$ & $121.4(9)$ \\
\hline $\mathrm{C}(74 \mathrm{~A})-\mathrm{C}(73 \mathrm{~A})-\mathrm{C}(72 \mathrm{~A})$ & $120.3(9)$ \\
\hline$C(75 A)-C(74 A)-C(73 A)$ & $119.8(8)$ \\
\hline$C(74 A)-C(75 A)-C(76 A)$ & $120.5(8)$ \\
\hline$C(75 A)-C(76 A)-C(71 A)$ & $120.8(7)$ \\
\hline$C(75 A)-C(76 A)-C(77 A)$ & $123.2(8)$ \\
\hline$C(71 A)-C(76 A)-C(77 A)$ & $116.0(7)$ \\
\hline $\mathrm{O}(3 \mathrm{~A})-\mathrm{C}(77 \mathrm{~A})-\mathrm{C}(76 \mathrm{~A})$ & $117.4(8)$ \\
\hline $\mathrm{O}(3 \mathrm{~A})-\mathrm{C}(77 \mathrm{~A})-\mathrm{C}(78 \mathrm{~A})$ & $119.5(8)$ \\
\hline $\mathrm{C}(76 \mathrm{~A})-\mathrm{C}(77 \mathrm{~A})-\mathrm{C}(78 \mathrm{~A})$ & $123.1(8)$ \\
\hline $\mathrm{C}(79 \mathrm{~A})-\mathrm{C}(78 \mathrm{~A})-\mathrm{C}(77 \mathrm{~A})$ & $121.1(8)$ \\
\hline $\mathrm{C}(78 \mathrm{~A})-\mathrm{C}(79 \mathrm{~A})-\mathrm{C}(80 \mathrm{~A})$ & $128.7(8)$ \\
\hline$C(81 A)-C(80 A)-C(85 A)$ & $119.2(6)$ \\
\hline$C(81 A)-C(80 A)-C(79 A)$ & $122.8(6)$ \\
\hline$C(85 A)-C(80 A)-C(79 A)$ & $118.0(6)$ \\
\hline$C(80 A)-C(81 A)-C(82 A)$ & $120.4(7)$ \\
\hline$C(83 A)-C(82 A)-C(81 A)$ & $119.1(7)$ \\
\hline $\mathrm{C}(84 \mathrm{~A})-\mathrm{C}(83 \mathrm{~A})-\mathrm{C}(82 \mathrm{~A})$ & $121.1(7)$ \\
\hline$C(83 A)-C(84 A)-C(85 A)$ & $119.7(7)$ \\
\hline$C(84 A)-C(85 A)-C(80 A)$ & $120.6(7)$ \\
\hline $\mathrm{C}(77 \mathrm{~A})-\mathrm{O}(3 \mathrm{~A})-\mathrm{Pd}(1)$ & $114.8(8)$ \\
\hline $\mathrm{Cl}(1)-\mathrm{C}(1 \mathrm{~S})-\mathrm{Cl}(2)$ & $111.37(18)$ \\
\hline $\mathrm{Cl}(3)-\mathrm{C}(2 \mathrm{~S})-\mathrm{Cl}(4)$ & $111.5(5)$ \\
\hline $\mathrm{Cl}(3 \mathrm{~A})-\mathrm{C}(2 \mathrm{SA})-\mathrm{Cl}(4 \mathrm{~A})$ & $114.1(8)$ \\
\hline $\mathrm{Cl}(3 \mathrm{~B})-\mathrm{C}(1 \mathrm{SB})-\mathrm{Cl}(4 \mathrm{~B})$ & $111.8(10)$ \\
\hline
\end{tabular}




\begin{tabular}{|c|c|}
\hline $\mathrm{O}(4)-\mathrm{S}(1)-\mathrm{O}(5)$ & $115.5(4)$ \\
\hline $\mathrm{O}(4)-\mathrm{S}(1)-\mathrm{O}(6)$ & $116.9(4)$ \\
\hline $\mathrm{O}(5)-\mathrm{S}(1)-\mathrm{O}(6)$ & $115.8(4)$ \\
\hline $\mathrm{O}(4)-\mathrm{S}(1)-\mathrm{C}(1)$ & $101.5(3)$ \\
\hline $\mathrm{O}(5)-\mathrm{S}(1)-\mathrm{C}(1)$ & $102.4(4)$ \\
\hline $\mathrm{O}(6)-\mathrm{S}(1)-\mathrm{C}(1)$ & $100.8(3)$ \\
\hline $\mathrm{F}(1)-\mathrm{C}(1)-\mathrm{F}(3)$ & $108.0(6)$ \\
\hline $\mathrm{F}(1)-\mathrm{C}(1)-\mathrm{F}(2)$ & $107.0(6)$ \\
\hline $\mathrm{F}(3)-\mathrm{C}(1)-\mathrm{F}(2)$ & $107.4(6)$ \\
\hline $\mathrm{F}(1)-\mathrm{C}(1)-\mathrm{S}(1)$ & $113.3(5)$ \\
\hline $\mathrm{F}(3)-\mathrm{C}(1)-\mathrm{S}(1)$ & $109.9(5)$ \\
\hline $\mathrm{F}(2)-\mathrm{C}(1)-\mathrm{S}(1)$ & $111.0(5)$ \\
\hline $\mathrm{O}(6 \mathrm{~A})-\mathrm{S}(1 \mathrm{~A})-\mathrm{O}(5 \mathrm{~A})$ & $117.9(7)$ \\
\hline $\mathrm{O}(6 \mathrm{~A})-\mathrm{S}(1 \mathrm{~A})-\mathrm{O}(4 \mathrm{~A})$ & $113.3(8)$ \\
\hline $\mathrm{O}(5 \mathrm{~A})-\mathrm{S}(1 \mathrm{~A})-\mathrm{O}(4 \mathrm{~A})$ & $113.8(7)$ \\
\hline $\mathrm{O}(6 \mathrm{~A})-\mathrm{S}(1 \mathrm{~A})-\mathrm{C}(1 \mathrm{~A})$ & $105.6(7)$ \\
\hline $\mathrm{O}(5 \mathrm{~A})-\mathrm{S}(1 \mathrm{~A})-\mathrm{C}(1 \mathrm{~A})$ & $103.9(5)$ \\
\hline $\mathrm{O}(4 \mathrm{~A})-\mathrm{S}(1 \mathrm{~A})-\mathrm{C}(1 \mathrm{~A})$ & $99.7(6)$ \\
\hline$F(3 A)-C(1 A)-F(1 A)$ & $110.0(10)$ \\
\hline$F(3 A)-C(1 A)-F(2 A)$ & $106.0(9)$ \\
\hline $\mathrm{F}(1 \mathrm{~A})-\mathrm{C}(1 \mathrm{~A})-\mathrm{F}(2 \mathrm{~A})$ & $110.4(9)$ \\
\hline$F(3 A)-C(1 A)-S(1 A)$ & $110.8(7)$ \\
\hline$F(1 A)-C(1 A)-S(1 A)$ & $112.0(10)$ \\
\hline$F(2 A)-C(1 A)-S(1 A)$ & $107.4(8)$ \\
\hline $\mathrm{O}(4 \mathrm{~B})-\mathrm{S}(1 \mathrm{~B})-\mathrm{O}(5 \mathrm{~B})$ & $115.5(8)$ \\
\hline $\mathrm{O}(4 \mathrm{~B})-\mathrm{S}(1 \mathrm{~B})-\mathrm{O}(6 \mathrm{~B})$ & $118.7(9)$ \\
\hline $\mathrm{O}(5 \mathrm{~B})-\mathrm{S}(1 \mathrm{~B})-\mathrm{O}(6 \mathrm{~B})$ & $114.4(8)$ \\
\hline $\mathrm{O}(4 \mathrm{~B})-\mathrm{S}(1 \mathrm{~B})-\mathrm{C}(1 \mathrm{~B})$ & $102.9(8)$ \\
\hline $\mathrm{O}(5 \mathrm{~B})-\mathrm{S}(1 \mathrm{~B})-\mathrm{C}(1 \mathrm{~B})$ & $100.5(7)$ \\
\hline $\mathrm{O}(6 \mathrm{~B})-\mathrm{S}(1 \mathrm{~B})-\mathrm{C}(1 \mathrm{~B})$ & $100.6(7)$ \\
\hline $\mathrm{F}(1 \mathrm{~B})-\mathrm{C}(1 \mathrm{~B})-\mathrm{F}(3 \mathrm{~B})$ & $109.8(12)$ \\
\hline $\mathrm{F}(1 \mathrm{~B})-\mathrm{C}(1 \mathrm{~B})-\mathrm{F}(2 \mathrm{~B})$ & $105.5(11)$ \\
\hline $\mathrm{F}(3 \mathrm{~B})-\mathrm{C}(1 \mathrm{~B})-\mathrm{F}(2 \mathrm{~B})$ & $106.4(11)$ \\
\hline $\mathrm{F}(1 \mathrm{~B})-\mathrm{C}(1 \mathrm{~B})-\mathrm{S}(1 \mathrm{~B})$ & $110.3(13)$ \\
\hline $\mathrm{F}(3 \mathrm{~B})-\mathrm{C}(1 \mathrm{~B})-\mathrm{S}(1 \mathrm{~B})$ & $114.4(11)$ \\
\hline
\end{tabular}


Symmetry transformations used to generate equivalent atoms:

Table 4. Anisotropic displacement parameters $\left(\AA^{2} \times 10^{3}\right)$ for 06249. The anisotropic displacement factor exponent takes the form: $-2 \mathrm{p}^{2}\left[\mathrm{~h}^{2} \mathrm{a} * 2 \mathrm{U}^{11}+\ldots+2 \mathrm{~h} \mathrm{k} \mathrm{a*} \mathrm{b}^{*} \mathrm{U}^{12}\right]$

\begin{tabular}{|c|c|c|c|c|c|c|}
\hline & $\mathrm{U}^{11}$ & $\mathrm{U}^{22}$ & $\mathrm{U}^{33}$ & $\mathrm{U}^{23}$ & $\mathrm{U}^{13}$ & $\mathrm{U}^{12}$ \\
\hline $\mathrm{P}(1)$ & $15(1)$ & $15(1)$ & $20(1)$ & $2(1)$ & $4(1)$ & $0(1)$ \\
\hline $\mathrm{C}(11)$ & $15(1)$ & $19(1)$ & $17(1)$ & $1(1)$ & $5(1)$ & $-2(1)$ \\
\hline$C(12)$ & $17(1)$ & $20(1)$ & $15(1)$ & $1(1)$ & $2(1)$ & $-2(1)$ \\
\hline$C(13)$ & $21(1)$ & $19(1)$ & $19(1)$ & $2(1)$ & $2(1)$ & $-1(1)$ \\
\hline$C(14)$ & $20(1)$ & $25(1)$ & $29(1)$ & $6(1)$ & $7(1)$ & $-3(1)$ \\
\hline$C(15)$ & $16(1)$ & $29(2)$ & $37(2)$ & $5(1)$ & $10(1)$ & $0(1)$ \\
\hline$C(16)$ & $19(1)$ & $23(1)$ & $28(1)$ & $4(1)$ & $7(1)$ & $2(1)$ \\
\hline$C(17)$ & $31(2)$ & $22(1)$ & $42(2)$ & $14(1)$ & $3(1)$ & $-4(1)$ \\
\hline $\mathrm{C}(21)$ & $20(1)$ & $16(1)$ & $27(1)$ & $3(1)$ & $9(1)$ & $0(1)$ \\
\hline$C(22)$ & $25(1)$ & $29(1)$ & $26(1)$ & $5(1)$ & $8(1)$ & $8(1)$ \\
\hline$C(23)$ & $28(2)$ & $34(2)$ & $37(2)$ & $13(1)$ & $12(1)$ & $11(1)$ \\
\hline$C(24)$ & $33(2)$ & $19(1)$ & $52(2)$ & 11(1) & $23(1)$ & $7(1)$ \\
\hline$C(25)$ & $35(2)$ & $21(1)$ & $45(2)$ & $-6(1)$ & $22(1)$ & $-2(1)$ \\
\hline$C(26)$ & $27(1)$ & $23(1)$ & $35(2)$ & $-4(1)$ & 11(1) & $-3(1)$ \\
\hline$C(31)$ & $19(1)$ & $16(1)$ & $27(1)$ & $0(1)$ & $1(1)$ & $-2(1)$ \\
\hline$C(32)$ & $20(1)$ & $25(1)$ & $33(2)$ & $3(1)$ & $2(1)$ & $-2(1)$ \\
\hline$C(33)$ & $20(1)$ & $34(2)$ & $53(2)$ & $2(2)$ & $-5(1)$ & $-5(1)$ \\
\hline$C(34)$ & $35(2)$ & $36(2)$ & $50(2)$ & $-7(2)$ & $-14(2)$ & $-7(1)$ \\
\hline$C(35)$ & $47(2)$ & $36(2)$ & $30(2)$ & $-10(1)$ & $-8(1)$ & $-5(1)$ \\
\hline$C(36)$ & $33(2)$ & $26(1)$ & $26(1)$ & $-3(1)$ & $3(1)$ & $-4(1)$ \\
\hline$C(41)$ & $14(1)$ & $17(1)$ & $23(1)$ & $7(1)$ & $1(1)$ & $0(1)$ \\
\hline$C(42)$ & $17(1)$ & $14(1)$ & $22(1)$ & $4(1)$ & $1(1)$ & $0(1)$ \\
\hline$C(43)$ & $22(1)$ & $21(1)$ & $24(1)$ & $5(1)$ & $-2(1)$ & $-2(1)$ \\
\hline$C(44)$ & $20(1)$ & $29(2)$ & $32(2)$ & $6(1)$ & $-8(1)$ & $-4(1)$ \\
\hline$C(45)$ & $14(1)$ & $32(2)$ & $42(2)$ & $10(1)$ & $1(1)$ & $-1(1)$ \\
\hline$C(46)$ & $17(1)$ & $25(1)$ & $29(1)$ & $9(1)$ & $6(1)$ & $2(1)$ \\
\hline$C(47)$ & $45(2)$ & $62(2)$ & $24(2)$ & $0(2)$ & $-9(1)$ & $-5(2)$ \\
\hline$P(2)$ & $14(1)$ & $17(1)$ & $19(1)$ & $4(1)$ & $5(1)$ & $2(1)$ \\
\hline$C(51)$ & $20(4)$ & $17(4)$ & $21(3)$ & $12(3)$ & $9(3)$ & $10(3)$ \\
\hline$C(52)$ & $24(3)$ & $25(6)$ & $26(4)$ & $10(3)$ & $7(3)$ & $7(3)$ \\
\hline
\end{tabular}




\begin{tabular}{|c|c|c|c|c|c|c|}
\hline$C(53)$ & $26(4)$ & $38(6)$ & $30(5)$ & $13(4)$ & $14(4)$ & $5(3)$ \\
\hline $\mathrm{C}(54)$ & $31(5)$ & $33(5)$ & $29(4)$ & $7(3)$ & $14(4)$ & $12(4)$ \\
\hline $\mathrm{C}(55)$ & $31(6)$ & $29(4)$ & $25(3)$ & $7(3)$ & $9(4)$ & $11(3)$ \\
\hline$C(56)$ & $26(6)$ & 23(4) & $23(3)$ & $5(2)$ & $9(3)$ & $7(3)$ \\
\hline $\mathrm{C}(51 \mathrm{~A})$ & 21(4) & $25(7)$ & 21(4) & $7(5)$ & $8(3)$ & $9(4)$ \\
\hline$C(52 A)$ & $17(4)$ & $28(7)$ & $28(5)$ & 6(4) & $10(3)$ & $7(4)$ \\
\hline$C(53 \mathrm{~A})$ & $19(4)$ & $37(7)$ & $36(6)$ & $5(4)$ & $12(4)$ & $1(4)$ \\
\hline$C(54 \mathrm{~A})$ & $23(5)$ & $41(7)$ & $30(5)$ & 4(4) & $14(4)$ & $7(5)$ \\
\hline$C(55 \mathrm{~A})$ & $30(7)$ & $27(5)$ & $29(4)$ & 1(3) & $13(5)$ & $8(4)$ \\
\hline$C(56 \mathrm{~A})$ & $25(7)$ & $24(5)$ & $24(4)$ & $10(3)$ & $10(4)$ & $8(4)$ \\
\hline $\mathrm{C}(61)$ & $16(1)$ & $16(1)$ & $19(1)$ & $-1(1)$ & $5(1)$ & $0(1)$ \\
\hline$C(62)$ & $19(1)$ & $22(1)$ & $24(1)$ & $2(1)$ & $2(1)$ & $1(1)$ \\
\hline$C(63)$ & $16(1)$ & $33(2)$ & $34(2)$ & $0(1)$ & $1(1)$ & $0(1)$ \\
\hline $\mathrm{C}(64)$ & $26(1)$ & $28(2)$ & $34(2)$ & $-1(1)$ & $4(1)$ & $-9(1)$ \\
\hline$C(65)$ & $32(1)$ & $16(1)$ & $29(1)$ & $3(1)$ & $4(1)$ & $-2(1)$ \\
\hline $\mathrm{C}(66)$ & $20(1)$ & 19(1) & $23(1)$ & $1(1)$ & $2(1)$ & $3(1)$ \\
\hline $\mathrm{O}(1)$ & $22(1)$ & 19(1) & $30(1)$ & $8(1)$ & $3(1)$ & $-2(1)$ \\
\hline $\mathrm{O}(2)$ & $29(1)$ & $34(1)$ & $21(1)$ & $1(1)$ & $-3(1)$ & $-3(1)$ \\
\hline $\operatorname{Pd}(1)$ & $15(1)$ & $16(1)$ & $19(1)$ & $5(1)$ & $4(1)$ & $1(1)$ \\
\hline$C(71)$ & $4(4)$ & 21(1) & $19(3)$ & $7(1)$ & $-4(3)$ & $2(1)$ \\
\hline$C(72)$ & $14(3)$ & $20(1)$ & $20(3)$ & $8(2)$ & $-4(4)$ & $3(1)$ \\
\hline$C(73)$ & $20(3)$ & $18(1)$ & $25(4)$ & 2(3) & $2(4)$ & $1(1)$ \\
\hline$C(74)$ & $22(2)$ & $17(2)$ & $26(5)$ & $3(3)$ & $4(4)$ & $-2(2)$ \\
\hline$C(75)$ & $20(3)$ & 20(3) & $20(4)$ & $5(2)$ & $3(4)$ & $0(3)$ \\
\hline$C(76)$ & $9(4)$ & $19(2)$ & $23(2)$ & $5(2)$ & $-1(3)$ & $-1(2)$ \\
\hline$C(77)$ & $12(3)$ & 19(3) & $22(2)$ & $6(1)$ & $3(2)$ & $1(4)$ \\
\hline$C(78)$ & $15(2)$ & $16(4)$ & $22(2)$ & $8(2)$ & $4(2)$ & $-4(4)$ \\
\hline$C(79)$ & $17(1)$ & 19(4) & $22(1)$ & $6(2)$ & $6(1)$ & $-2(4)$ \\
\hline $\mathrm{C}(80)$ & $17(1)$ & $26(3)$ & $24(1)$ & $2(2)$ & $6(1)$ & $-1(3)$ \\
\hline $\mathrm{C}(81)$ & $27(3)$ & $30(3)$ & $22(2)$ & $2(2)$ & $7(2)$ & $0(3)$ \\
\hline $\mathrm{C}(82)$ & $41(3)$ & $40(3)$ & $22(2)$ & $0(3)$ & $6(2)$ & $-6(3)$ \\
\hline $\mathrm{C}(83)$ & $40(3)$ & $46(5)$ & $27(3)$ & $-9(3)$ & $11(3)$ & $-10(4)$ \\
\hline $\mathrm{C}(84)$ & $45(3)$ & $38(3)$ & $40(3)$ & $-11(3)$ & $14(3)$ & $-11(3)$ \\
\hline$C(85)$ & $34(3)$ & $31(3)$ & $36(3)$ & $-3(3)$ & $17(3)$ & $-10(3)$ \\
\hline $\mathrm{O}(3)$ & $22(6)$ & 19(3) & $20(1)$ & $6(1)$ & $6(1)$ & $-3(3)$ \\
\hline$C(71 \mathrm{~A})$ & $4(4)$ & 21(1) & $19(3)$ & $7(1)$ & $-4(3)$ & $2(1)$ \\
\hline
\end{tabular}




\begin{tabular}{|c|c|c|c|c|c|c|}
\hline$C(72 A)$ & $14(3)$ & $20(1)$ & $20(3)$ & $8(2)$ & $-4(4)$ & $3(1)$ \\
\hline$C(73 A)$ & 20(3) & $18(1)$ & $25(4)$ & 2(3) & $2(4)$ & $1(1)$ \\
\hline $\mathrm{C}(74 \mathrm{~A})$ & $22(2)$ & $17(2)$ & $26(5)$ & $3(3)$ & $4(4)$ & $-2(2)$ \\
\hline$C(75 A)$ & $20(3)$ & $20(3)$ & $20(4)$ & $5(2)$ & $3(4)$ & $0(3)$ \\
\hline$C(76 \mathrm{~A})$ & $9(4)$ & $19(2)$ & $23(2)$ & $5(2)$ & $-1(3)$ & $-1(2)$ \\
\hline$C(77 \mathrm{~A})$ & $12(3)$ & $19(3)$ & $22(2)$ & $6(1)$ & $3(2)$ & $1(4)$ \\
\hline $\mathrm{C}(78 \mathrm{~A})$ & $15(2)$ & $16(4)$ & $22(2)$ & $8(2)$ & $4(2)$ & $-4(4)$ \\
\hline$C(79 A)$ & $17(1)$ & $19(4)$ & $22(1)$ & $6(2)$ & $6(1)$ & $-2(4)$ \\
\hline$C(80 A)$ & $17(1)$ & $26(3)$ & $24(1)$ & $2(2)$ & $6(1)$ & $-1(3)$ \\
\hline$C(81 \mathrm{~A})$ & $35(4)$ & $28(3)$ & $26(3)$ & $3(2)$ & $6(3)$ & $7(3)$ \\
\hline $\mathrm{C}(82 \mathrm{~A})$ & $52(4)$ & $34(4)$ & $25(3)$ & $3(3)$ & $10(4)$ & $17(4)$ \\
\hline$C(83 \mathrm{~A})$ & $46(4)$ & $43(4)$ & $24(3)$ & $-4(3)$ & $5(3)$ & $11(4)$ \\
\hline$C(84 A)$ & $36(3)$ & $36(4)$ & $31(3)$ & $-4(3)$ & $8(3)$ & $-3(3)$ \\
\hline$C(85 \mathrm{~A})$ & $25(3)$ & $30(3)$ & $28(3)$ & $-1(3)$ & $8(2)$ & $-4(3)$ \\
\hline $\mathrm{O}(3 \mathrm{~A})$ & $22(6)$ & $19(3)$ & $20(1)$ & $6(1)$ & $6(1)$ & $-3(3)$ \\
\hline $\mathrm{Cl}(1)$ & $52(1)$ & $74(1)$ & $40(1)$ & $18(1)$ & $-8(1)$ & $6(1)$ \\
\hline$C(1 S)$ & $41(2)$ & $31(2)$ & $41(2)$ & $5(1)$ & $6(1)$ & $2(1)$ \\
\hline $\mathrm{Cl}(2)$ & $74(1)$ & $35(1)$ & $92(1)$ & $-19(1)$ & $31(1)$ & $-5(1)$ \\
\hline $\mathrm{Cl}(3)$ & $61(2)$ & $137(4)$ & $72(2)$ & $33(3)$ & $23(2)$ & $27(2)$ \\
\hline$C(2 S)$ & $108(7)$ & $105(9)$ & $53(6)$ & $4(6)$ & $41(5)$ & $34(5)$ \\
\hline $\mathrm{Cl}(4)$ & $95(2)$ & $59(2)$ & $46(1)$ & $19(1)$ & $31(1)$ & $19(1)$ \\
\hline $\mathrm{Cl}(3 \mathrm{~A})$ & $279(11)$ & $86(5)$ & 204(9) & $79(5)$ & $12(8)$ & $5(6)$ \\
\hline $\mathrm{C}(2 \mathrm{SA})$ & 191(14) & $105(10)$ & $69(12)$ & $35(9)$ & $18(12)$ & $50(8)$ \\
\hline $\mathrm{Cl}(4 \mathrm{~A})$ & $48(2)$ & $43(2)$ & $55(2)$ & $-4(1)$ & $0(1)$ & $-2(1)$ \\
\hline $\mathrm{Cl}(3 \mathrm{~B})$ & $252(16)$ & $155(10)$ & $200(14)$ & $12(10)$ & $-14(12)$ & $29(10)$ \\
\hline $\mathrm{C}(1 \mathrm{SB})$ & 181(13) & $140(10)$ & $54(11)$ & $-40(10)$ & $32(11)$ & $84(11)$ \\
\hline $\mathrm{Cl}(4 \mathrm{~B})$ & $296(18)$ & $380(20)$ & $124(15)$ & $62(15)$ & 11(13) & $-92(18)$ \\
\hline $\mathrm{S}(1)$ & $26(1)$ & $21(1)$ & $37(1)$ & $-5(1)$ & $6(1)$ & $0(1)$ \\
\hline $\mathrm{O}(4)$ & $24(2)$ & $34(3)$ & $34(3)$ & $9(2)$ & $10(2)$ & $4(2)$ \\
\hline $\mathrm{O}(5)$ & $36(2)$ & $37(3)$ & $82(4)$ & $14(3)$ & $26(3)$ & $17(2)$ \\
\hline $\mathrm{O}(6)$ & $37(2)$ & $43(3)$ & $79(4)$ & $-18(3)$ & $24(3)$ & $-18(2)$ \\
\hline $\mathrm{C}(1)$ & $59(4)$ & $45(4)$ & $35(3)$ & $9(2)$ & $11(3)$ & $2(3)$ \\
\hline $\mathrm{F}(1)$ & $112(5)$ & $65(3)$ & $63(3)$ & $29(3)$ & $32(3)$ & $7(3)$ \\
\hline $\mathrm{F}(2)$ & $87(3)$ & $62(3)$ & $56(3)$ & $-1(2)$ & $38(3)$ & $17(3)$ \\
\hline$F(3)$ & $86(3)$ & 101(4) & $53(3)$ & $-11(3)$ & $-10(3)$ & $-30(3)$ \\
\hline $\mathrm{S}(1 \mathrm{~A})$ & $54(2)$ & $21(2)$ & $49(2)$ & $3(2)$ & $31(2)$ & $0(1)$ \\
\hline
\end{tabular}




\begin{tabular}{lllllll}
$\mathrm{O}(4 \mathrm{~A})$ & $77(5)$ & $37(5)$ & $49(5)$ & $-13(4)$ & $3(4)$ & $-6(4)$ \\
$\mathrm{O}(5 \mathrm{~A})$ & $34(5)$ & $22(3)$ & $41(6)$ & $-5(3)$ & $14(4)$ & $4(3)$ \\
$\mathrm{O}(6 \mathrm{~A})$ & $67(5)$ & $34(5)$ & $149(9)$ & $-26(7)$ & $68(6)$ & $-7(5)$ \\
$\mathrm{C}(1 \mathrm{~A})$ & $88(6)$ & $60(4)$ & $63(5)$ & $30(4)$ & $5(3)$ & $-9(5)$ \\
$\mathrm{F}(1 \mathrm{~A})$ & $162(7)$ & $146(8)$ & $106(7)$ & $31(7)$ & $-65(7)$ & $38(8)$ \\
$\mathrm{F}(2 \mathrm{~A})$ & $192(10)$ & $49(4)$ & $136(8)$ & $53(4)$ & $44(6)$ & $-18(5)$ \\
$\mathrm{F}(3 \mathrm{~A})$ & $109(5)$ & $103(6)$ & $57(5)$ & $13(5)$ & $50(4)$ & $-47(5)$ \\
$\mathrm{S}(1 \mathrm{~B})$ & $23(2)$ & $30(3)$ & $31(3)$ & $4(2)$ & $2(2)$ & $-2(2)$ \\
$\mathrm{O}(4 \mathrm{~B})$ & $47(6)$ & $27(8)$ & $33(7)$ & $2(6)$ & $17(6)$ & $-15(7)$ \\
$\mathrm{O}(5 \mathrm{~B})$ & $15(7)$ & $27(5)$ & $31(9)$ & $8(5)$ & $2(6)$ & $-7(6)$ \\
$\mathrm{O}(6 \mathrm{~B})$ & $28(5)$ & $49(8)$ & $69(8)$ & $48(7)$ & $-22(5)$ & $-26(5)$ \\
$\mathrm{C}(1 \mathrm{~B})$ & $26(6)$ & $30(5)$ & $46(6)$ & $-9(5)$ & $9(4)$ & $11(6)$ \\
$\mathrm{F}(1 \mathrm{~B})$ & $33(5)$ & $52(10)$ & $67(10)$ & $-41(7)$ & $-12(6)$ & $26(7)$ \\
$\mathrm{F}(2 \mathrm{~B})$ & $80(9)$ & $36(5)$ & $30(7)$ & $-3(4)$ & $-2(6)$ & $1(6)$ \\
$\mathrm{F}(3 \mathrm{~B})$ & $30(6)$ & $40(7)$ & $34(5)$ & $3(5)$ & $3(5)$ & $16(6)$ \\
\hline
\end{tabular}

Table 5. Hydrogen coordinates $\left(\times 10^{4}\right)$ and isotropic displacement parameters $\left(\AA^{2} \times 10^{3}\right)$ for 06249.

\begin{tabular}{|c|c|c|c|c|}
\hline & $\mathrm{x}$ & $\mathrm{y}$ & $\mathrm{z}$ & $\mathrm{U}(\mathrm{eq})$ \\
\hline $\mathrm{H}(14)$ & -68 & 1716 & 1561 & 29 \\
\hline $\mathrm{H}(15)$ & -949 & 3325 & 1361 & 32 \\
\hline $\mathrm{H}(16)$ & -98 & 4848 & 1140 & 27 \\
\hline $\mathrm{H}(17 \mathrm{~A})$ & 1029 & 586 & 1918 & 48 \\
\hline $\mathrm{H}(17 \mathrm{~B})$ & 1862 & -192 & 1748 & 48 \\
\hline $\mathrm{H}(17 \mathrm{C})$ & 871 & 196 & 1464 & 48 \\
\hline $\mathrm{H}(22)$ & 326 & 6099 & 435 & 31 \\
\hline $\mathrm{H}(23)$ & -991 & 7389 & 449 & 39 \\
\hline $\mathrm{H}(24)$ & -1065 & 8503 & 1008 & 40 \\
\hline $\mathrm{H}(25)$ & 150 & 8296 & 1558 & 39 \\
\hline $\mathrm{H}(26)$ & 1475 & 7013 & 1549 & 33 \\
\hline $\mathrm{H}(32)$ & 4024 & 5763 & 880 & 31 \\
\hline $\mathrm{H}(33)$ & 5501 & 6232 & 1300 & 43 \\
\hline $\mathrm{H}(34)$ & 5441 & 6403 & 1977 & 50 \\
\hline $\mathrm{H}(35)$ & 3919 & 6083 & 2249 & 46 \\
\hline $\mathrm{H}(36)$ & 2435 & 5580 & 1837 & 35 \\
\hline
\end{tabular}


S48

\begin{tabular}{|c|c|c|c|c|}
\hline $\mathrm{H}(44)$ & 5195 & 2940 & 1870 & 33 \\
\hline $\mathrm{H}(45)$ & 5800 & 2580 & 1268 & 35 \\
\hline $\mathrm{H}(46)$ & 4686 & 2527 & 680 & 29 \\
\hline $\mathrm{H}(47 \mathrm{~A})$ & 4304 & 2625 & 2407 & 67 \\
\hline $\mathrm{H}(47 \mathrm{~B})$ & 3576 & 3533 & 2571 & 67 \\
\hline $\mathrm{H}(47 \mathrm{C})$ & 4467 & 3906 & 2316 & 67 \\
\hline $\mathrm{H}(52)$ & 4276 & 3759 & 123 & 30 \\
\hline $\mathrm{H}(53)$ & 5073 & 3271 & -432 & 37 \\
\hline $\mathrm{H}(54)$ & 4457 & 1794 & -834 & 36 \\
\hline $\mathrm{H}(55)$ & 3065 & 769 & -667 & 33 \\
\hline $\mathrm{H}(56)$ & 2268 & 1234 & -108 & 28 \\
\hline $\mathrm{H}(52 \mathrm{~A})$ & 4228 & 3972 & 170 & 28 \\
\hline $\mathrm{H}(53 \mathrm{~A})$ & 5199 & 3564 & -344 & 36 \\
\hline $\mathrm{H}(54 \mathrm{~A})$ & 4764 & 2077 & -763 & 37 \\
\hline $\mathrm{H}(55 \mathrm{~A})$ & 3405 & 924 & -656 & 33 \\
\hline $\mathrm{H}(56 \mathrm{~A})$ & 2496 & 1238 & -107 & 28 \\
\hline $\mathrm{H}(62)$ & 351 & 3111 & 289 & 26 \\
\hline $\mathrm{H}(63)$ & -977 & 1825 & 278 & 33 \\
\hline $\mathrm{H}(64)$ & -633 & 14 & 478 & 35 \\
\hline $\mathrm{H}(65)$ & 1037 & -538 & 690 & 30 \\
\hline $\mathrm{H}(66)$ & 2377 & 733 & 707 & 25 \\
\hline $\mathrm{H}(72)$ & 2589 & 7475 & 731 & 22 \\
\hline $\mathrm{H}(73)$ & 3191 & 9105 & 470 & 25 \\
\hline $\mathrm{H}(74)$ & 3487 & 9214 & -191 & 26 \\
\hline $\mathrm{H}(75)$ & 3158 & 7699 & -607 & 24 \\
\hline $\mathrm{H}(78)$ & 2714 & 6300 & -984 & 21 \\
\hline $\mathrm{H}(79)$ & 2342 & 4041 & -899 & 23 \\
\hline $\mathrm{H}(81)$ & 2742 & 6117 & -1633 & 31 \\
\hline $\mathrm{H}(82)$ & 2764 & 5772 & -2308 & 41 \\
\hline $\mathrm{H}(83)$ & 2437 & 4007 & -2560 & 44 \\
\hline $\mathrm{H}(84)$ & 2092 & 2565 & -2146 & 48 \\
\hline $\mathrm{H}(85)$ & 2123 & 2876 & -1466 & 39 \\
\hline $\mathrm{H}(72 \mathrm{~A})$ & 2477 & 7528 & 644 & 22 \\
\hline $\mathrm{H}(73 \mathrm{~A})$ & 3112 & 9097 & 348 & 25 \\
\hline $\mathrm{H}(74 \mathrm{~A})$ & 3425 & 9055 & -317 & 26 \\
\hline $\mathrm{H}(75 \mathrm{~A})$ & 3085 & 7461 & -690 & 24 \\
\hline
\end{tabular}




\begin{tabular}{|c|c|c|c|c|}
\hline $\mathrm{H}(78 \mathrm{~A})$ & 2618 & 5980 & -1036 & 21 \\
\hline $\mathrm{H}(79 \mathrm{~A})$ & 2348 & 3745 & -884 & 23 \\
\hline $\mathrm{H}(81 \mathrm{~A})$ & 2517 & 5636 & -1699 & 35 \\
\hline $\mathrm{H}(82 \mathrm{~A})$ & 2452 & 5117 & -2369 & 44 \\
\hline $\mathrm{H}(83 \mathrm{~A})$ & 2312 & 3251 & -2540 & 45 \\
\hline $\mathrm{H}(84 \mathrm{~A})$ & 2261 & 1906 & -2054 & 41 \\
\hline $\mathrm{H}(85 \mathrm{~A})$ & 2304 & 2419 & -1390 & 33 \\
\hline $\mathrm{H}(1 \mathrm{~S} 1)$ & 4879 & 10263 & 1471 & 45 \\
\hline $\mathrm{H}(1 \mathrm{~S} 2)$ & 3745 & 10763 & 1433 & 45 \\
\hline $\mathrm{H}(2 \mathrm{~S} 1)$ & 9415 & 2065 & 2354 & 103 \\
\hline $\mathrm{H}(2 \mathrm{~S} 2)$ & 8844 & 2998 & 2076 & 103 \\
\hline $\mathrm{H}(2 \mathrm{~S} 3)$ & 11114 & 3895 & 2210 & 146 \\
\hline $\mathrm{H}(2 \mathrm{~S} 4)$ & 11163 & 4540 & 2623 & 146 \\
\hline H(1S3) & 9594 & 4099 & 2633 & 148 \\
\hline H(1S4) & 9204 & 3722 & 2189 & 148 \\
\hline
\end{tabular}



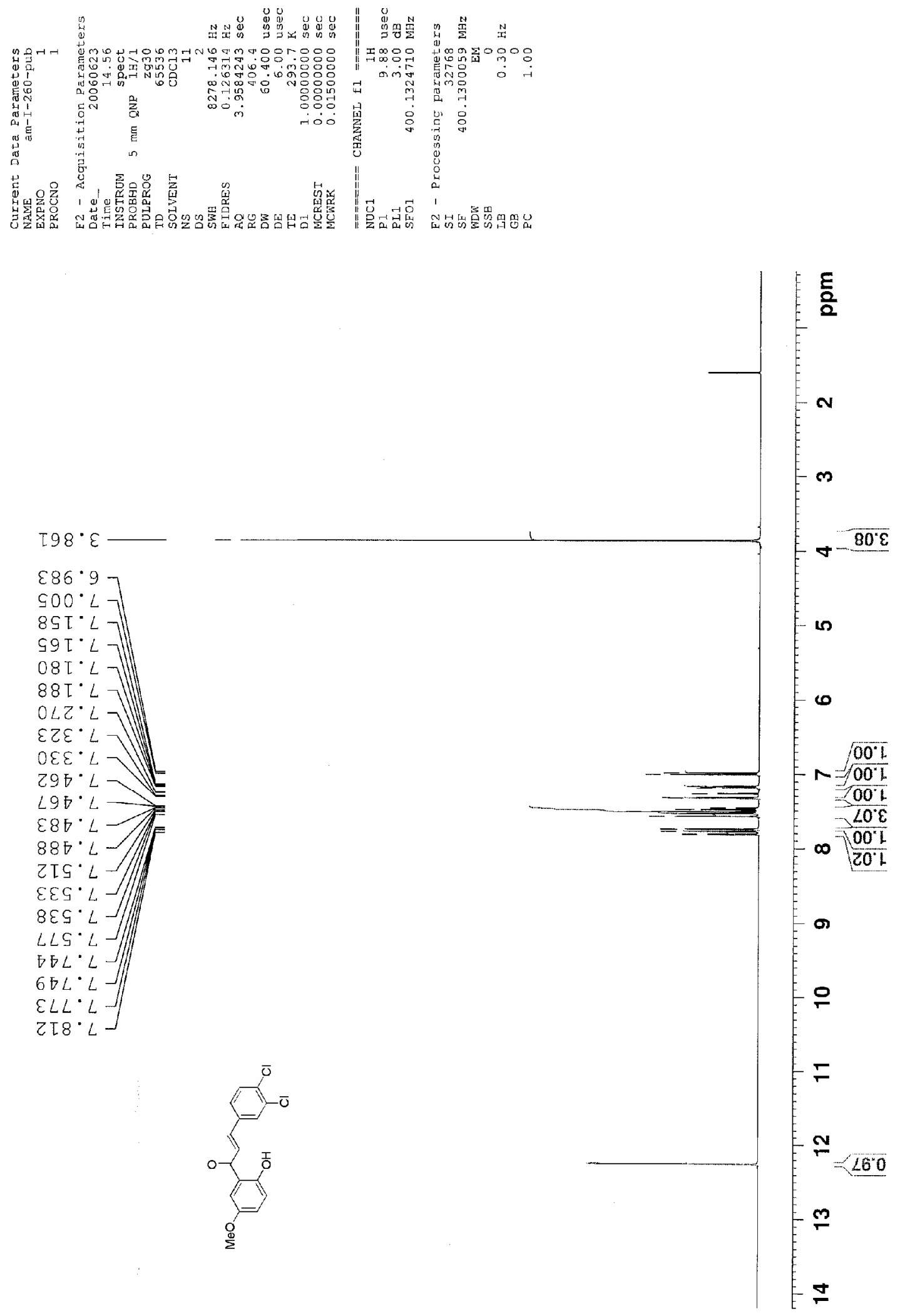

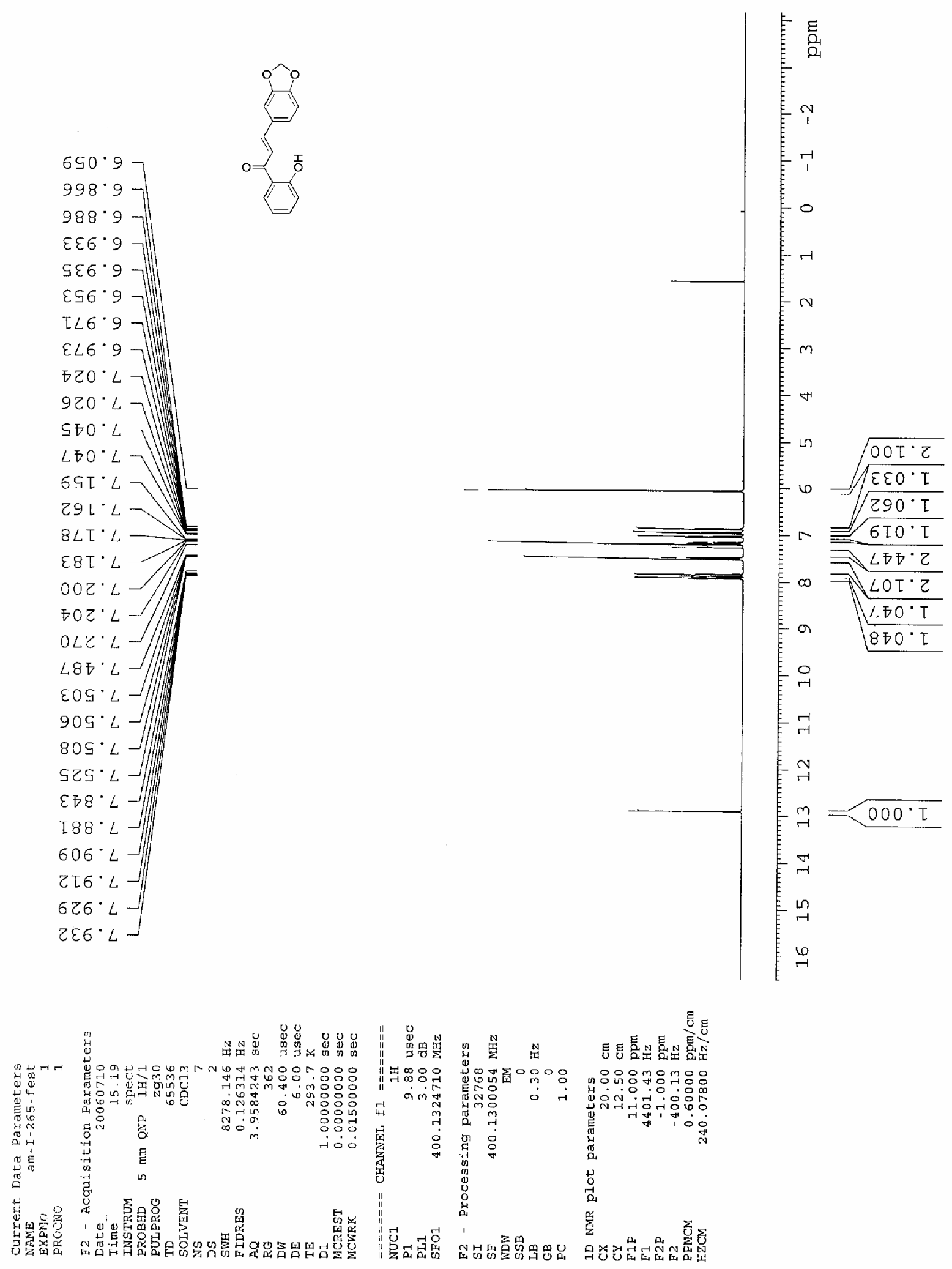

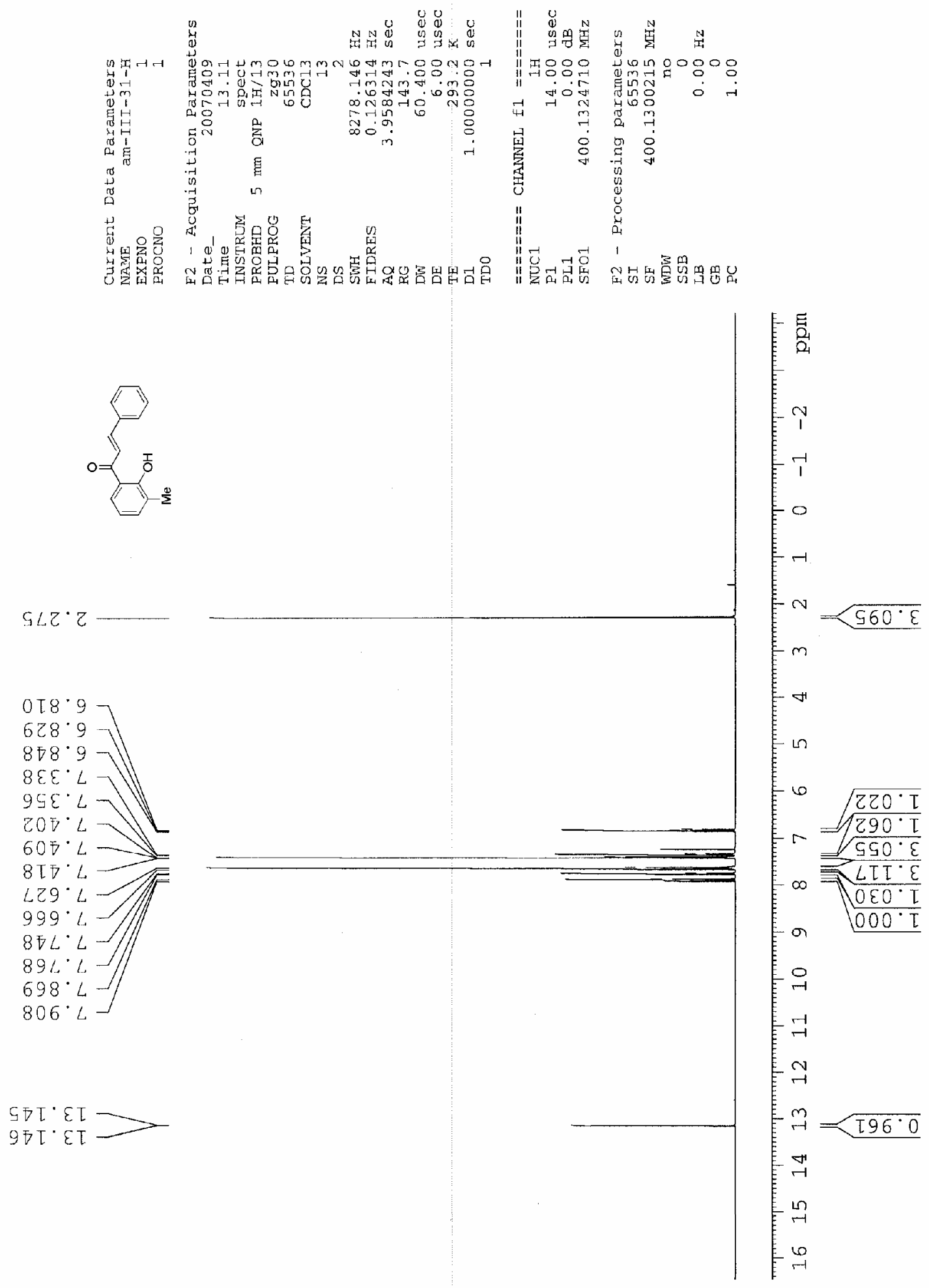

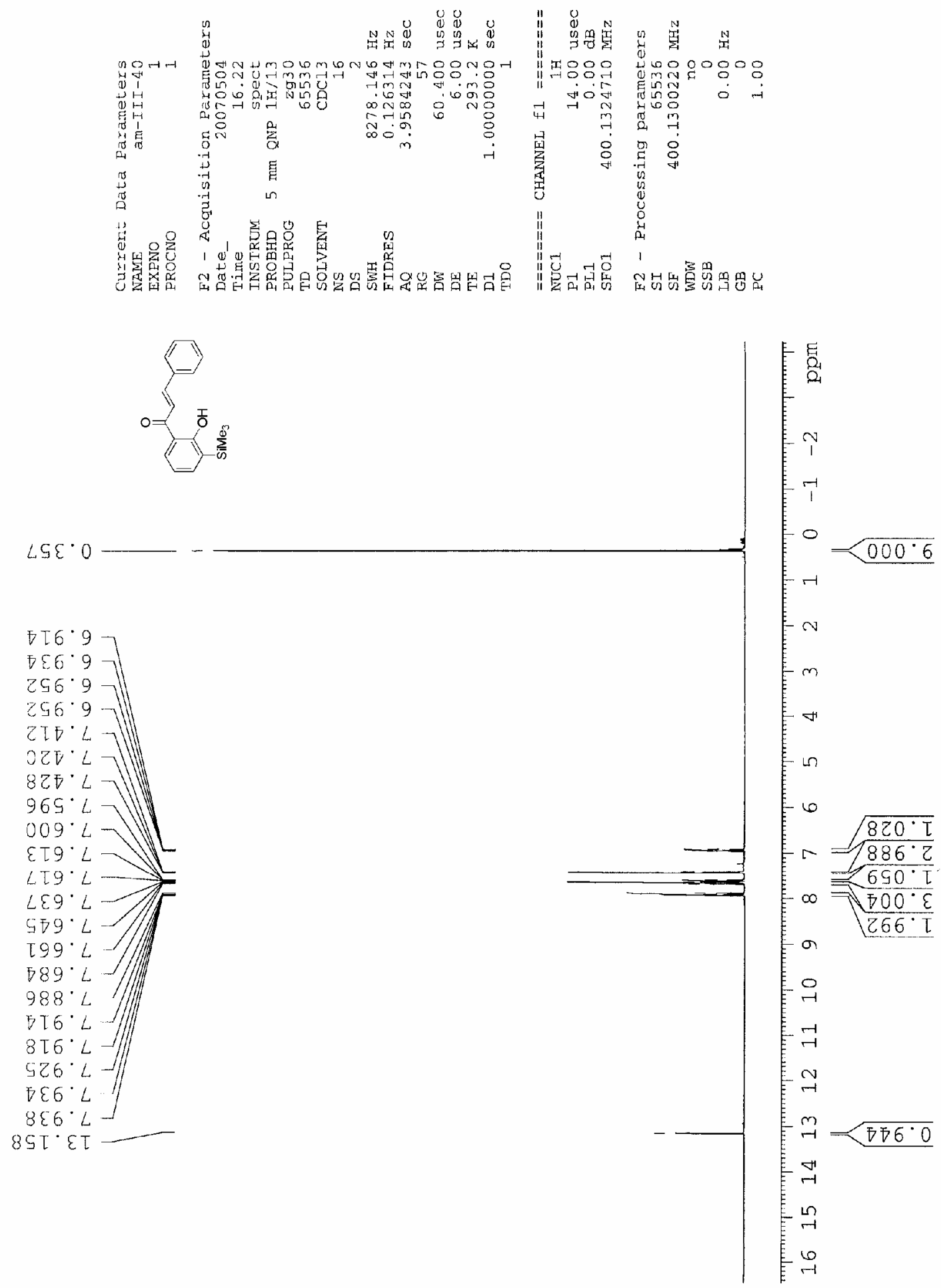


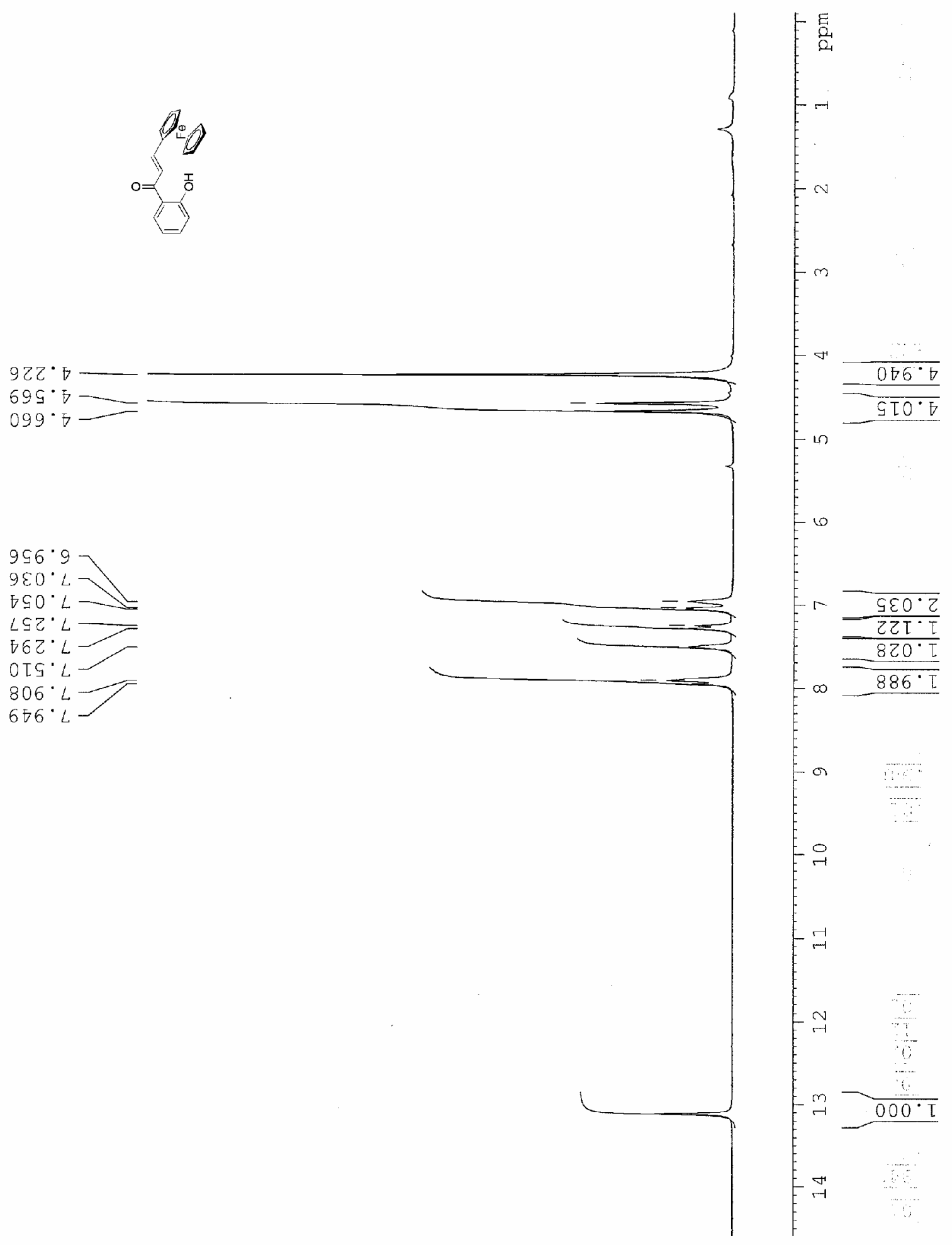



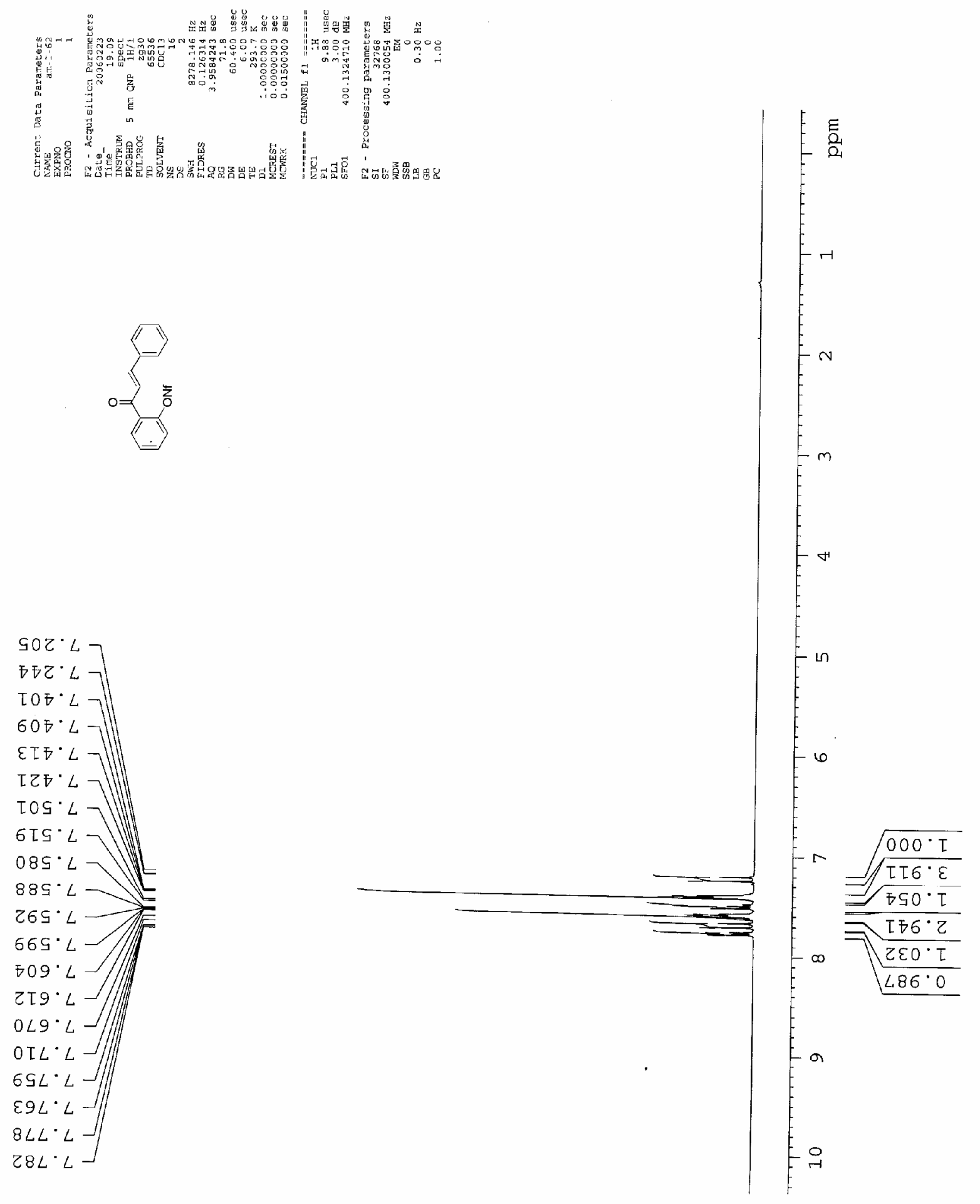


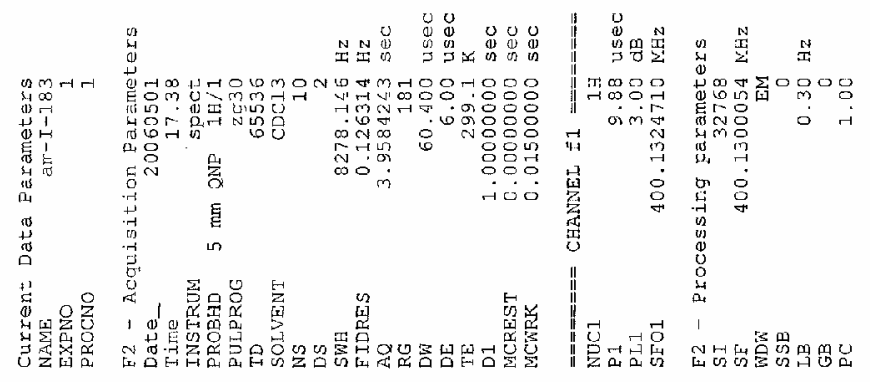

$8 \angle 8^{\circ} \varepsilon$
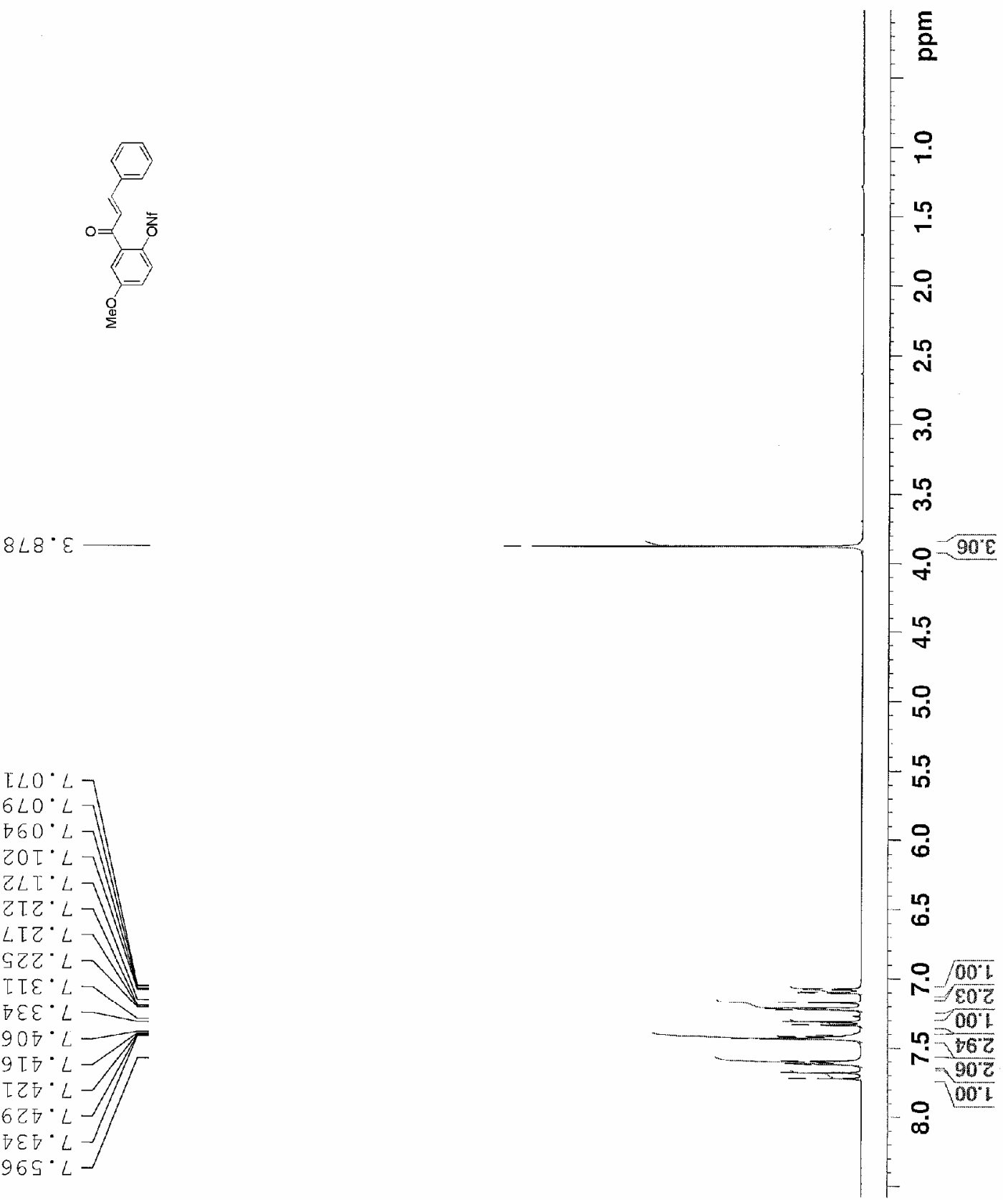

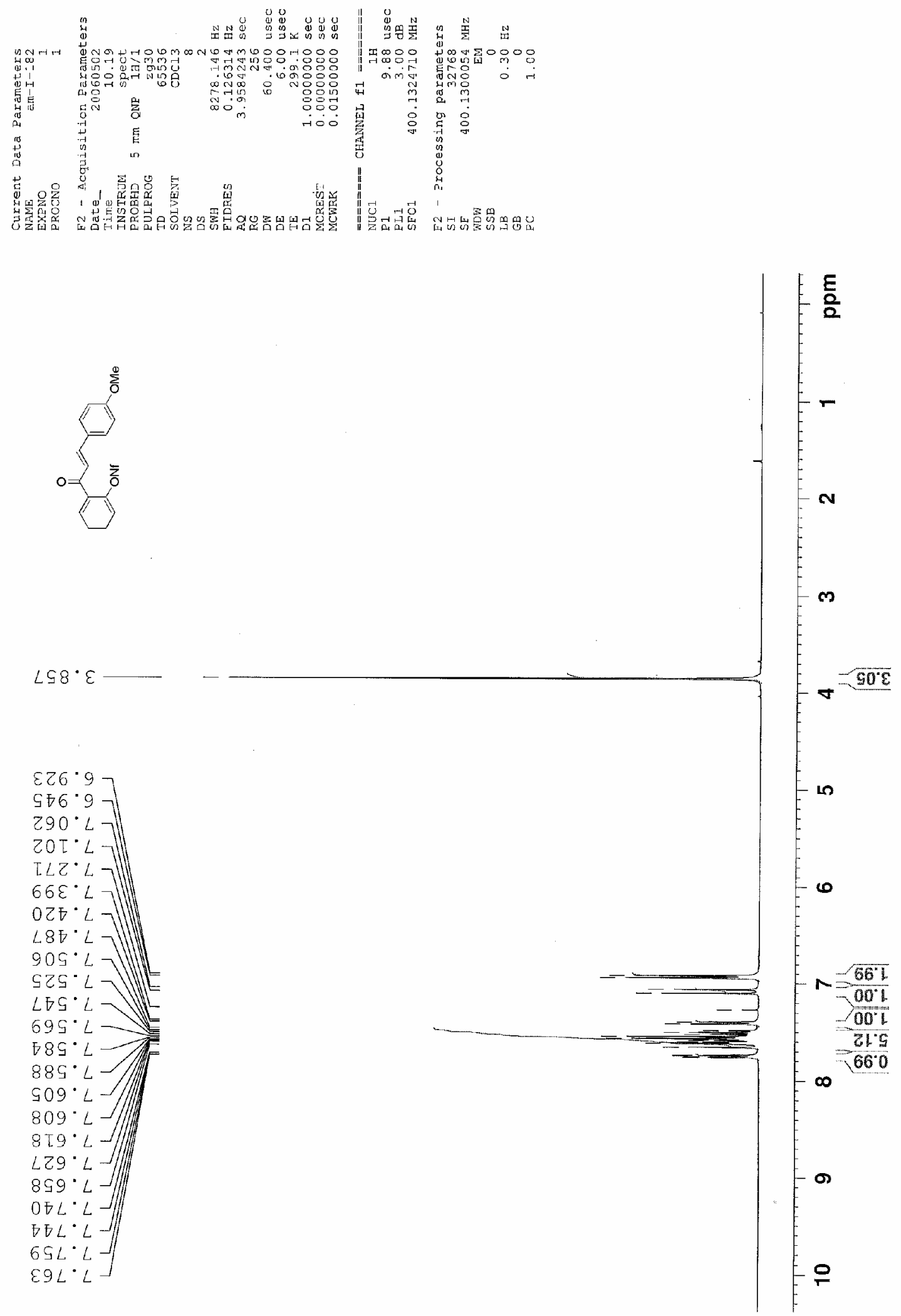


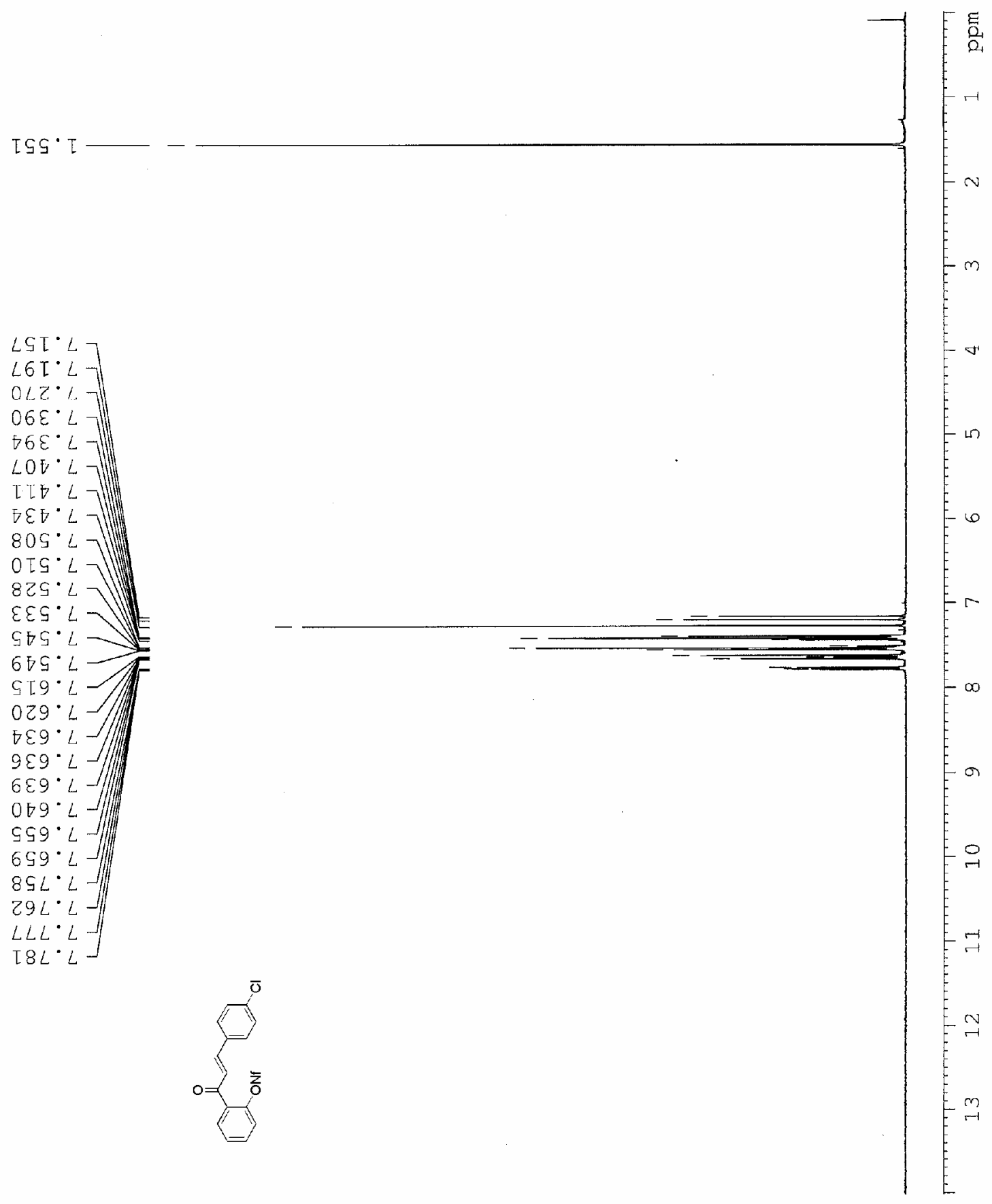



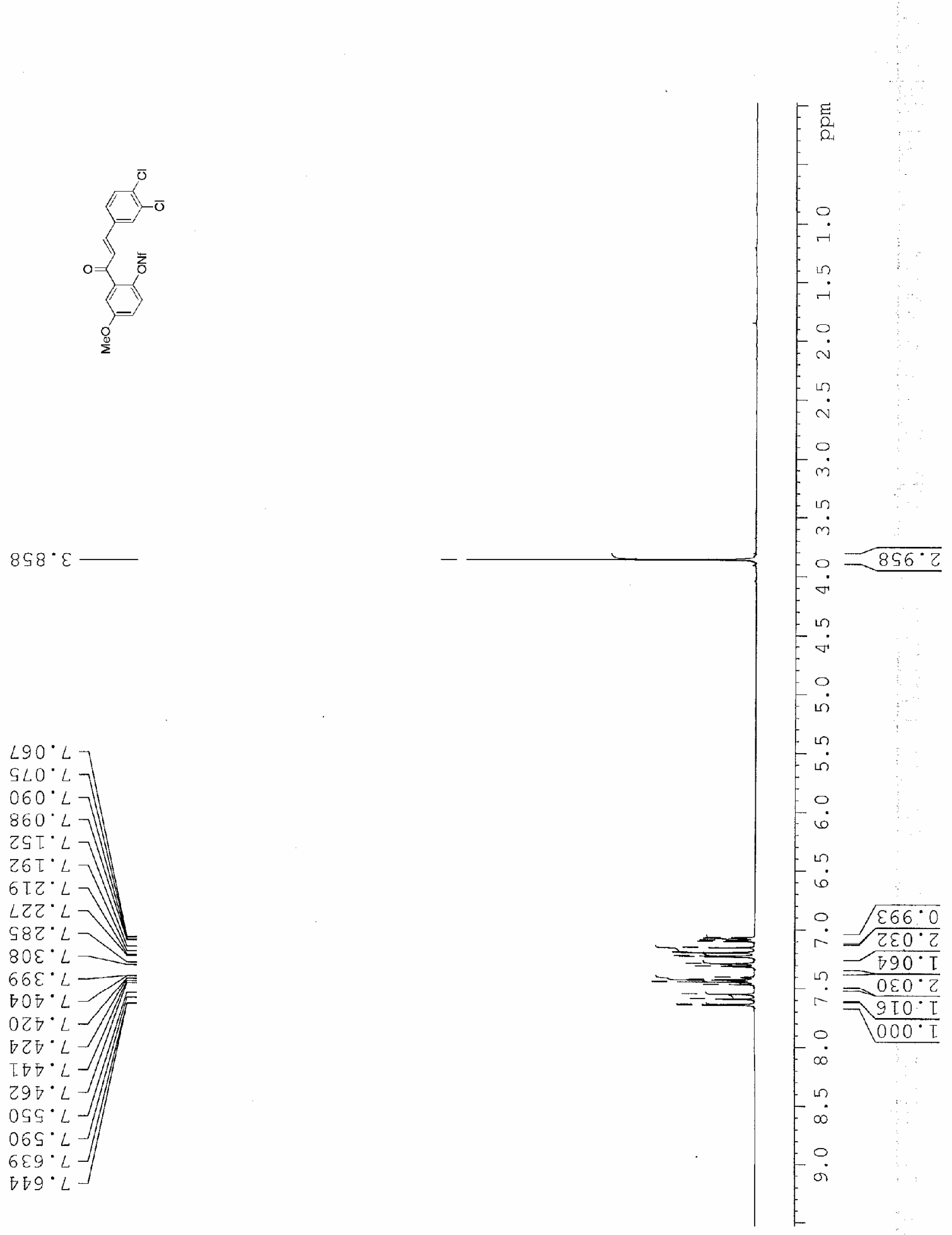

$\angle 90^{\circ} \mathrm{L}-$

$5 \angle 0^{\circ} L$

$\left.060^{\circ} \mathrm{L}\right]$

$\left.860^{\circ} \mathrm{L}\right]$

$\left.29 \tau^{\circ} L\right]$

$26 \mathrm{I}^{\circ}$

$6 \mathrm{TZ} \cdot$

$\angle Z Z^{\circ}$

$982^{\circ} L \frown$

$80 \varepsilon^{\circ} \mathrm{L}$

$66 \varepsilon^{\circ} L-\mathrm{T}$

O乙币. $L$

$\square Z \nabla \cdot L$

柿 $\cdot L-$

$290 \cdot L J$

$09^{\circ} L J$

$069^{\circ} \mathrm{L}-$

$6 \varepsilon 9 \cdot L$

$\square \nabla 9^{\circ} \mathrm{L}$ 

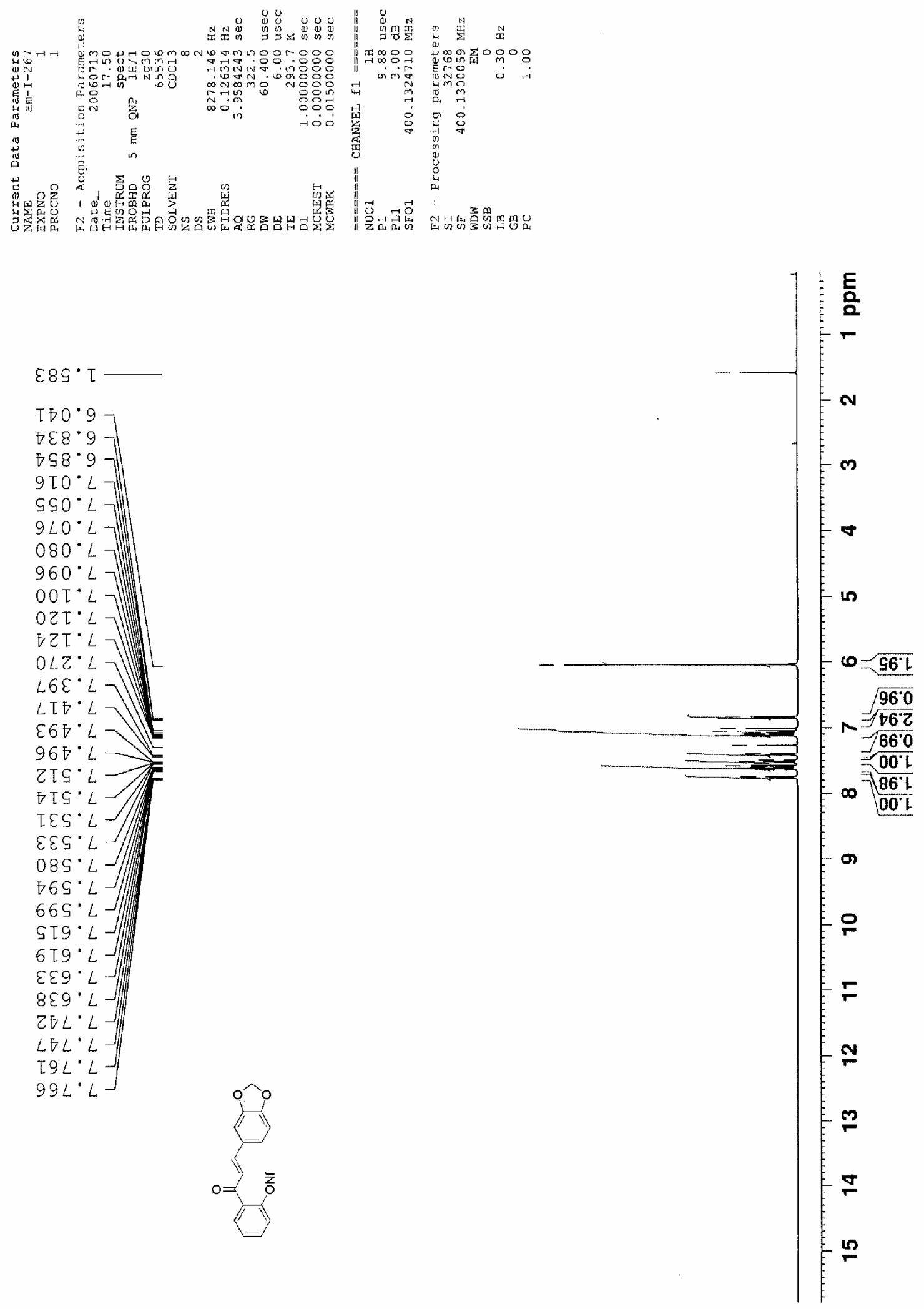


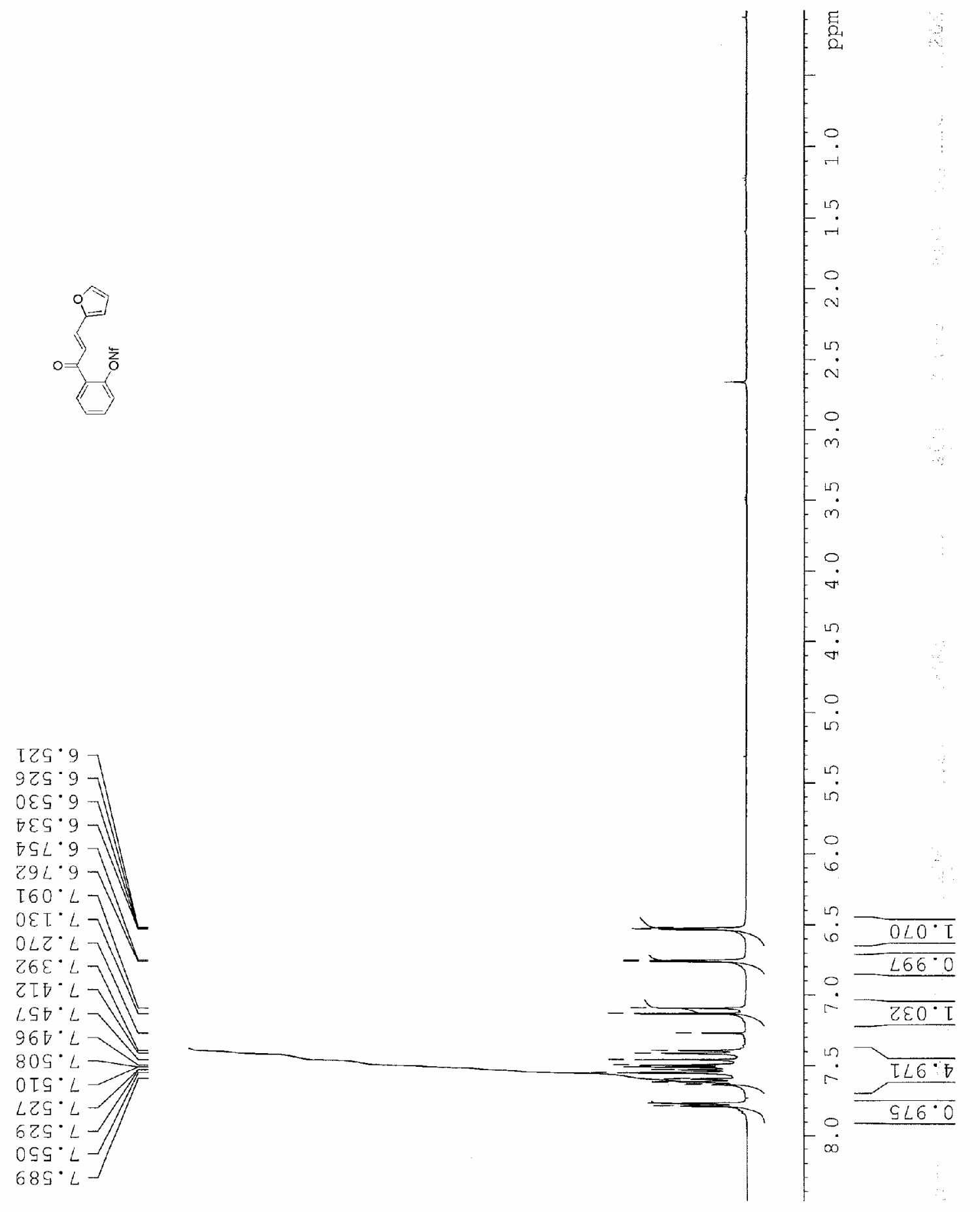



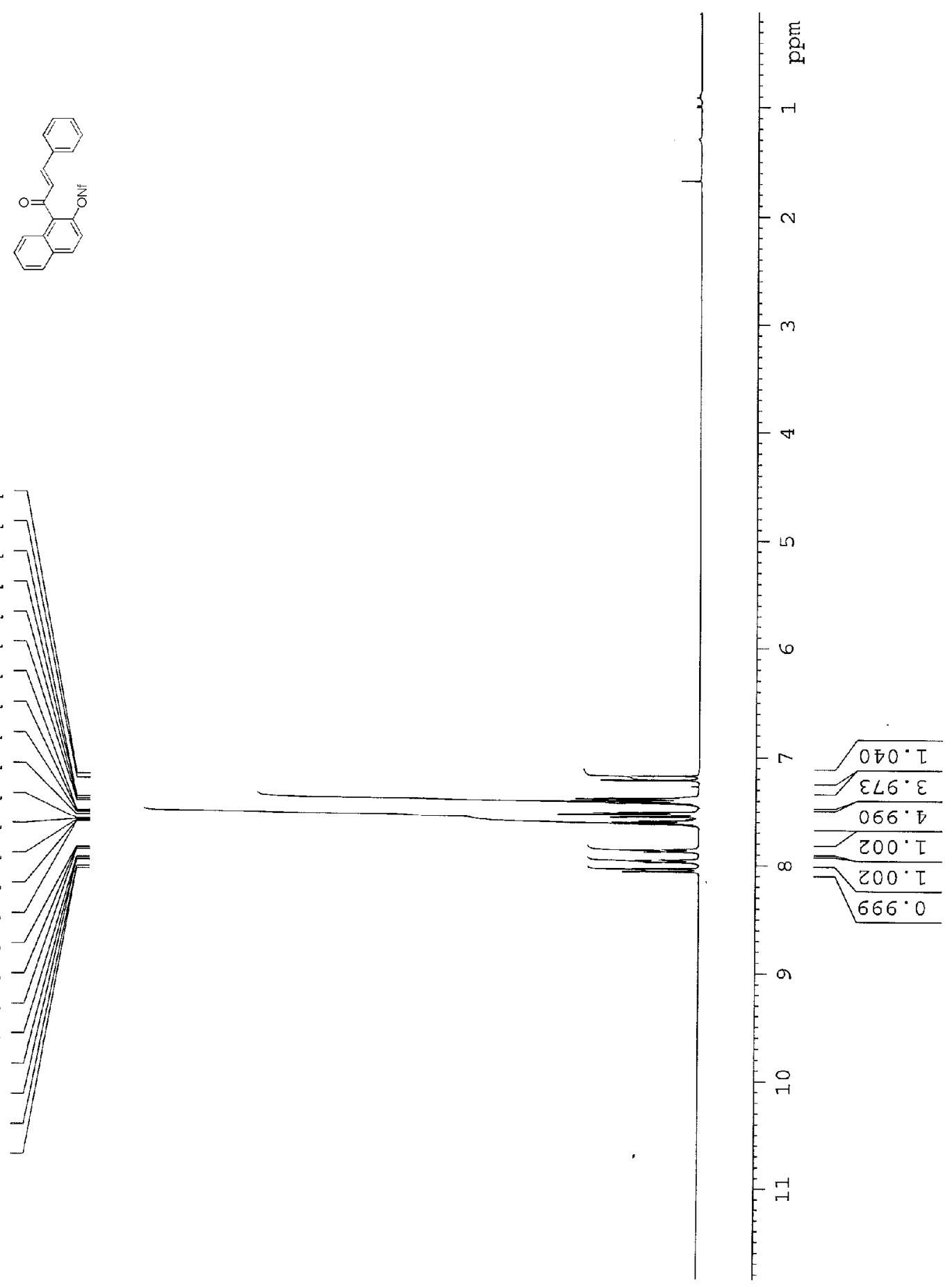


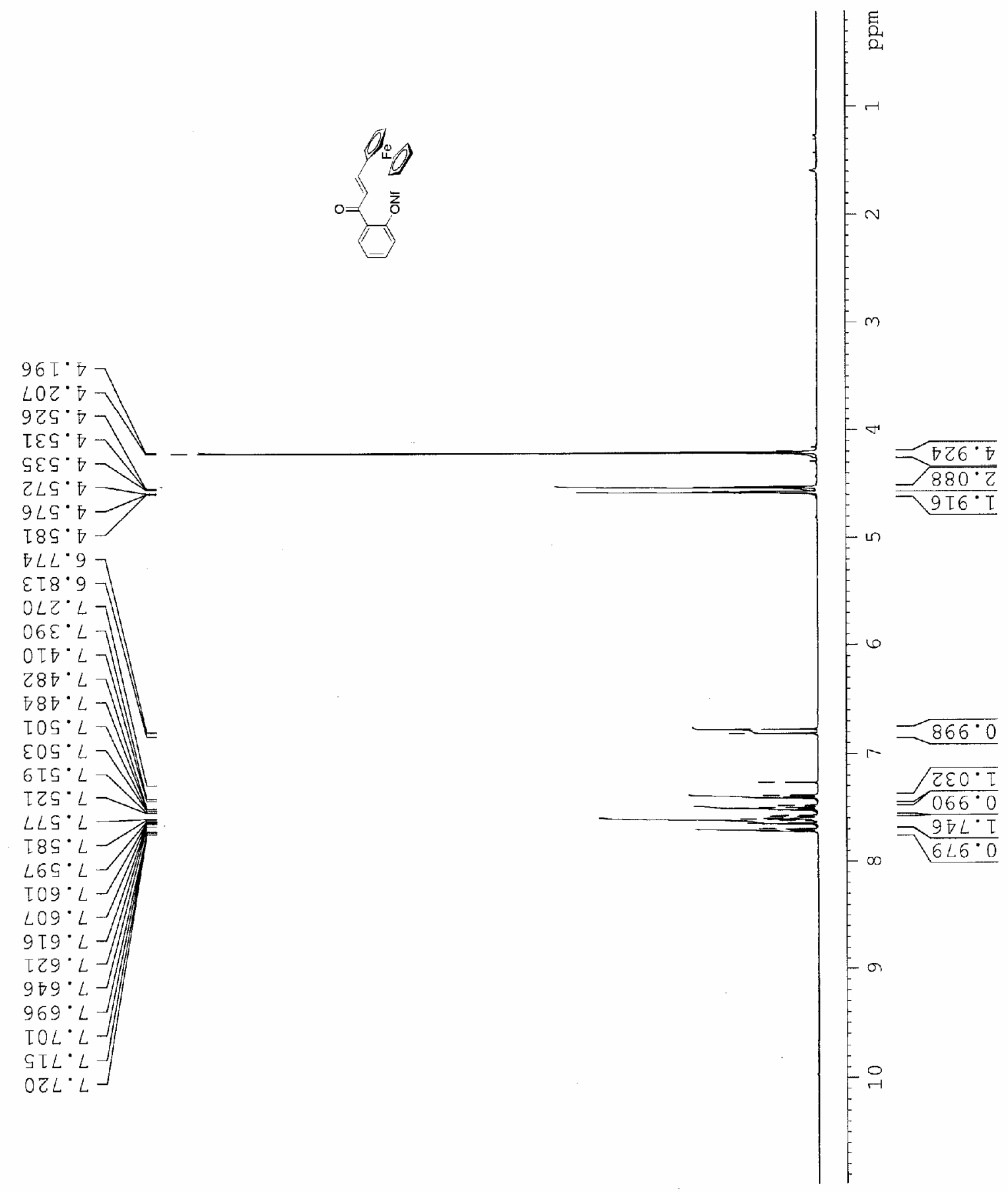



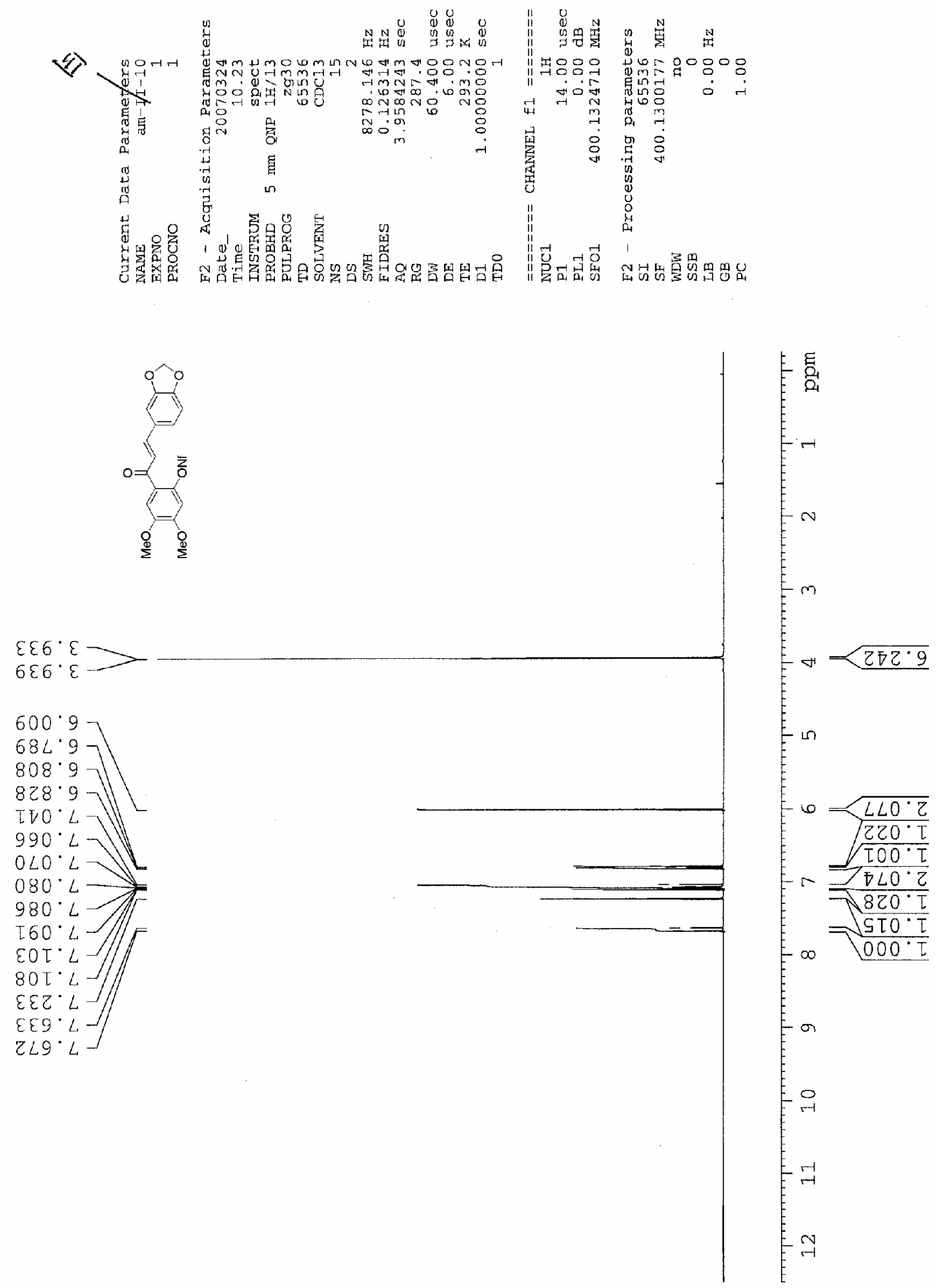

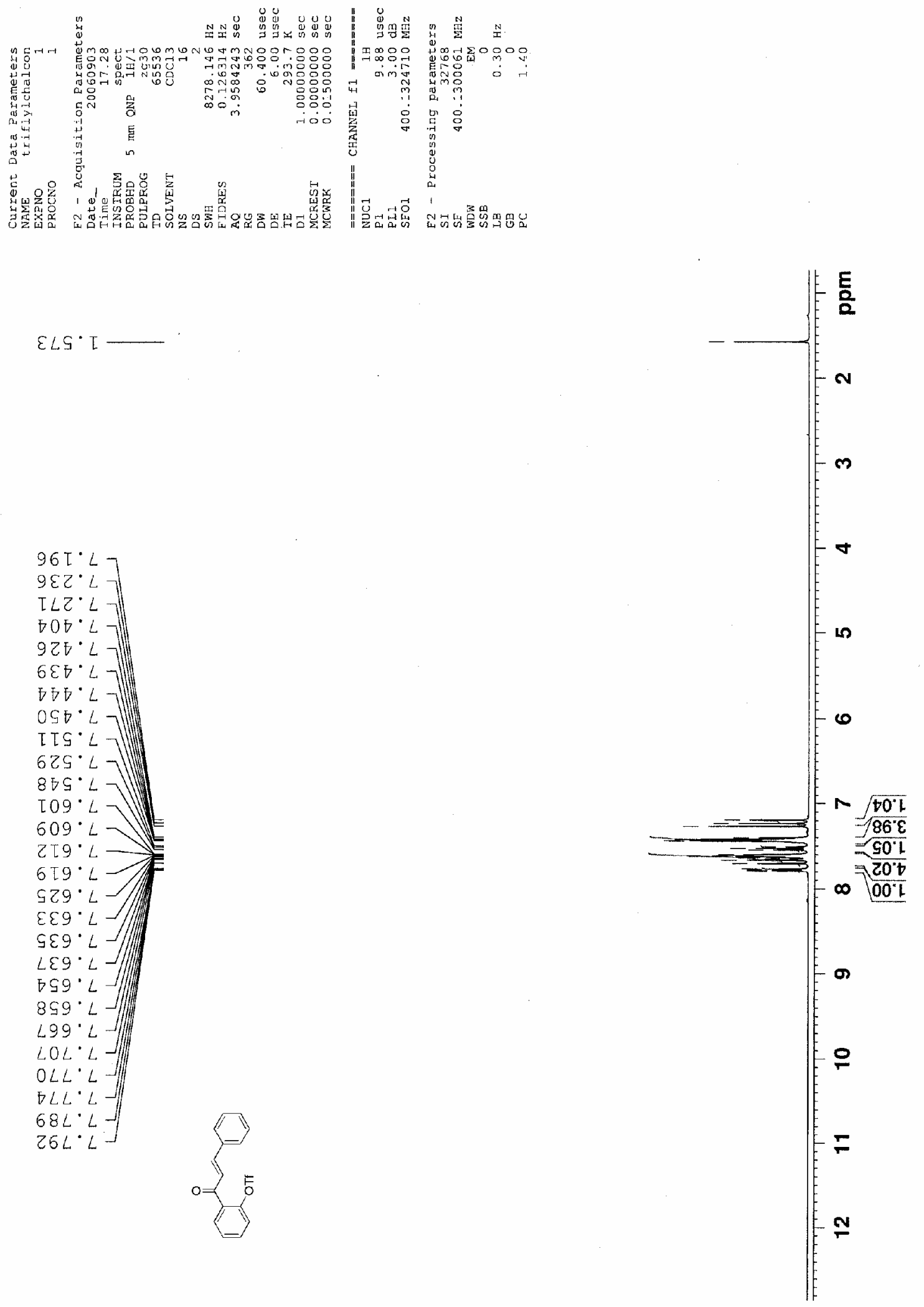

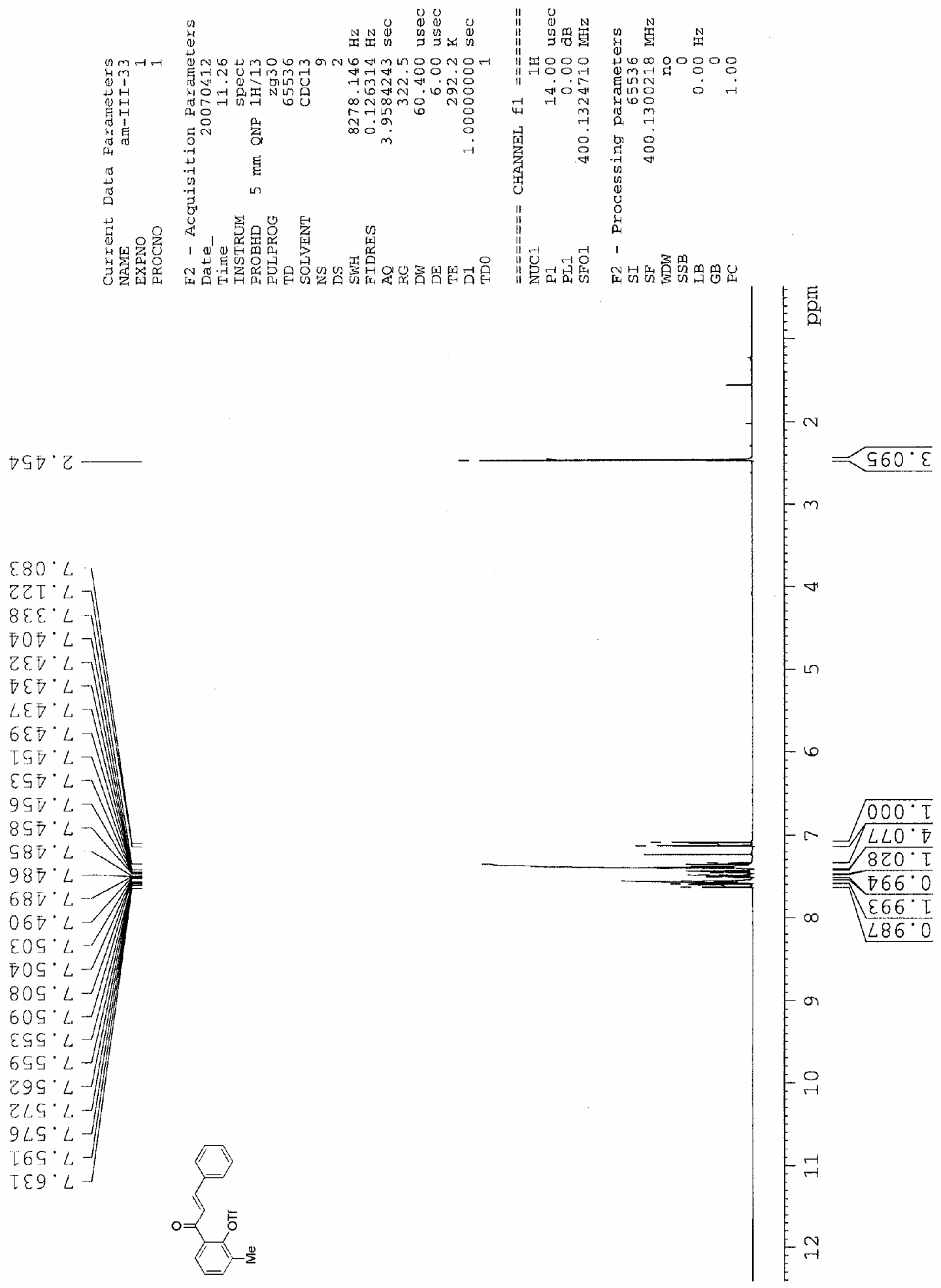

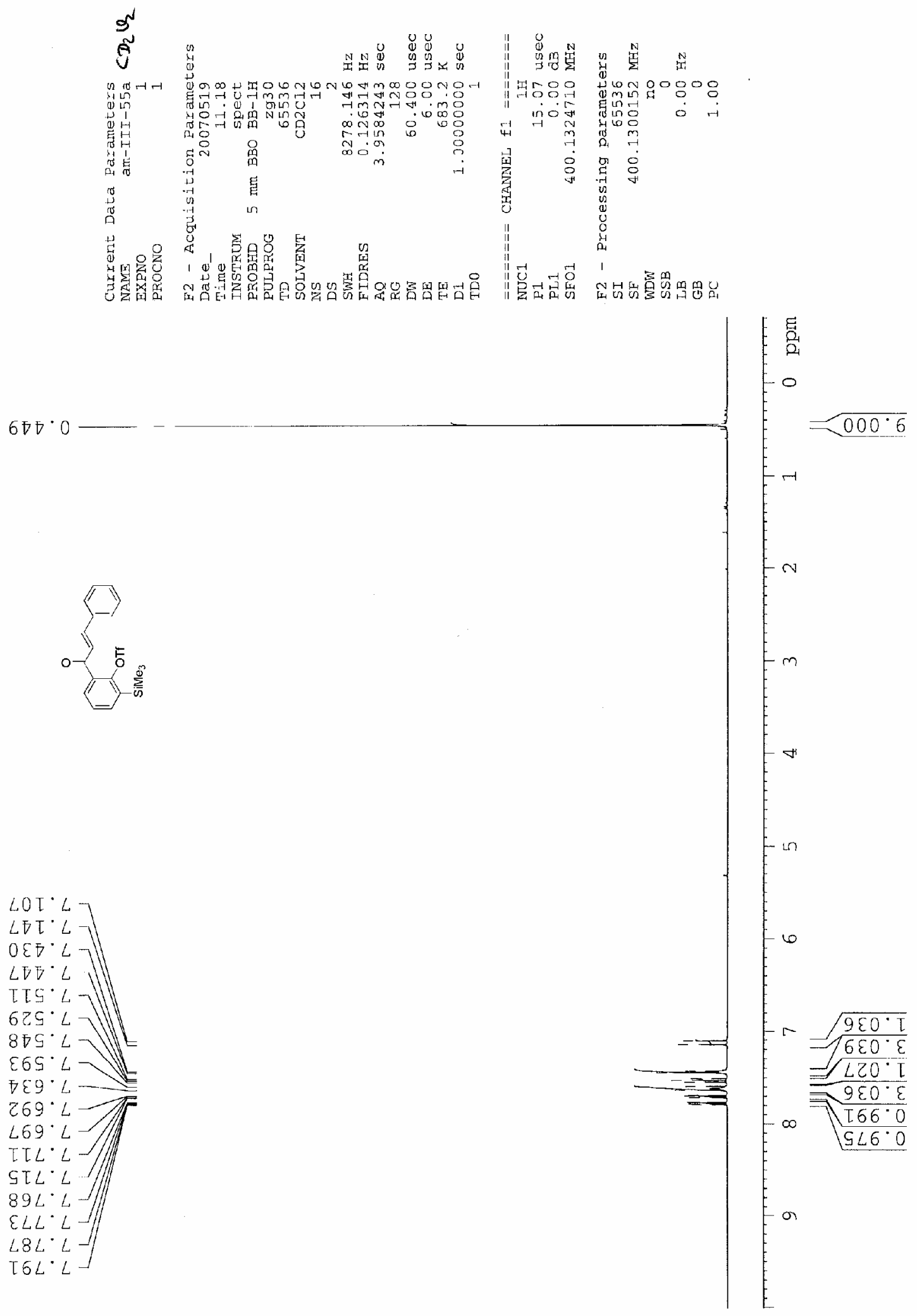

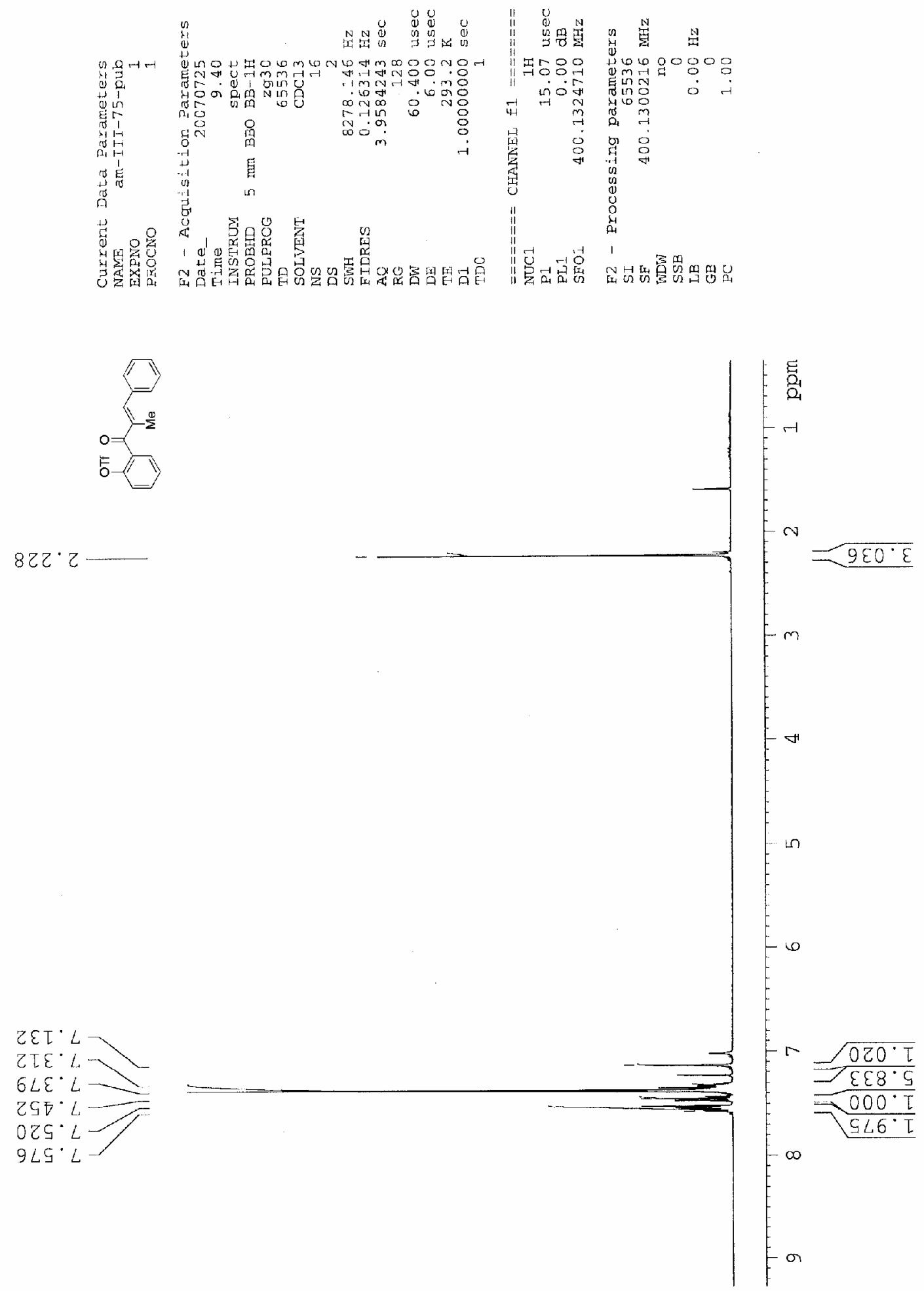

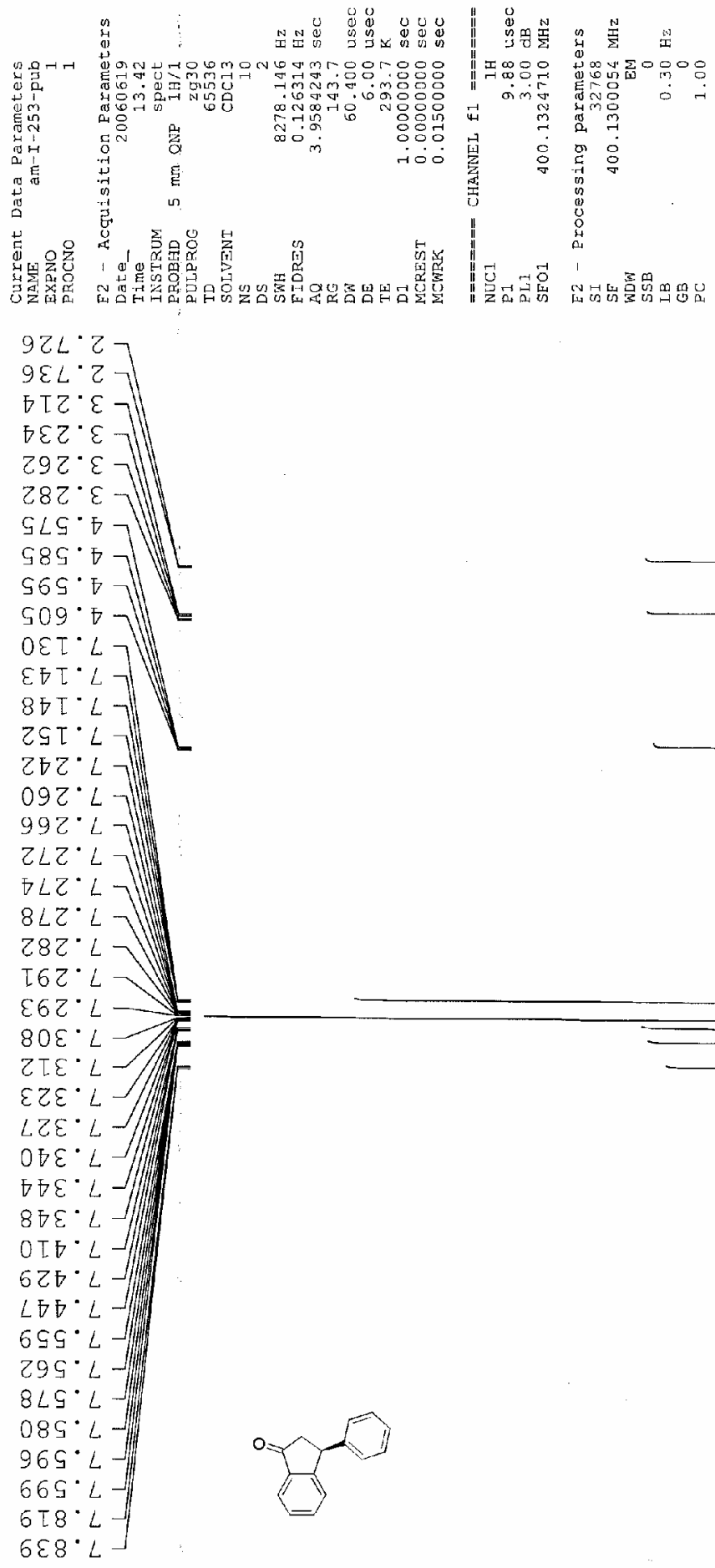
$86^{\circ} \cdot 5 b$
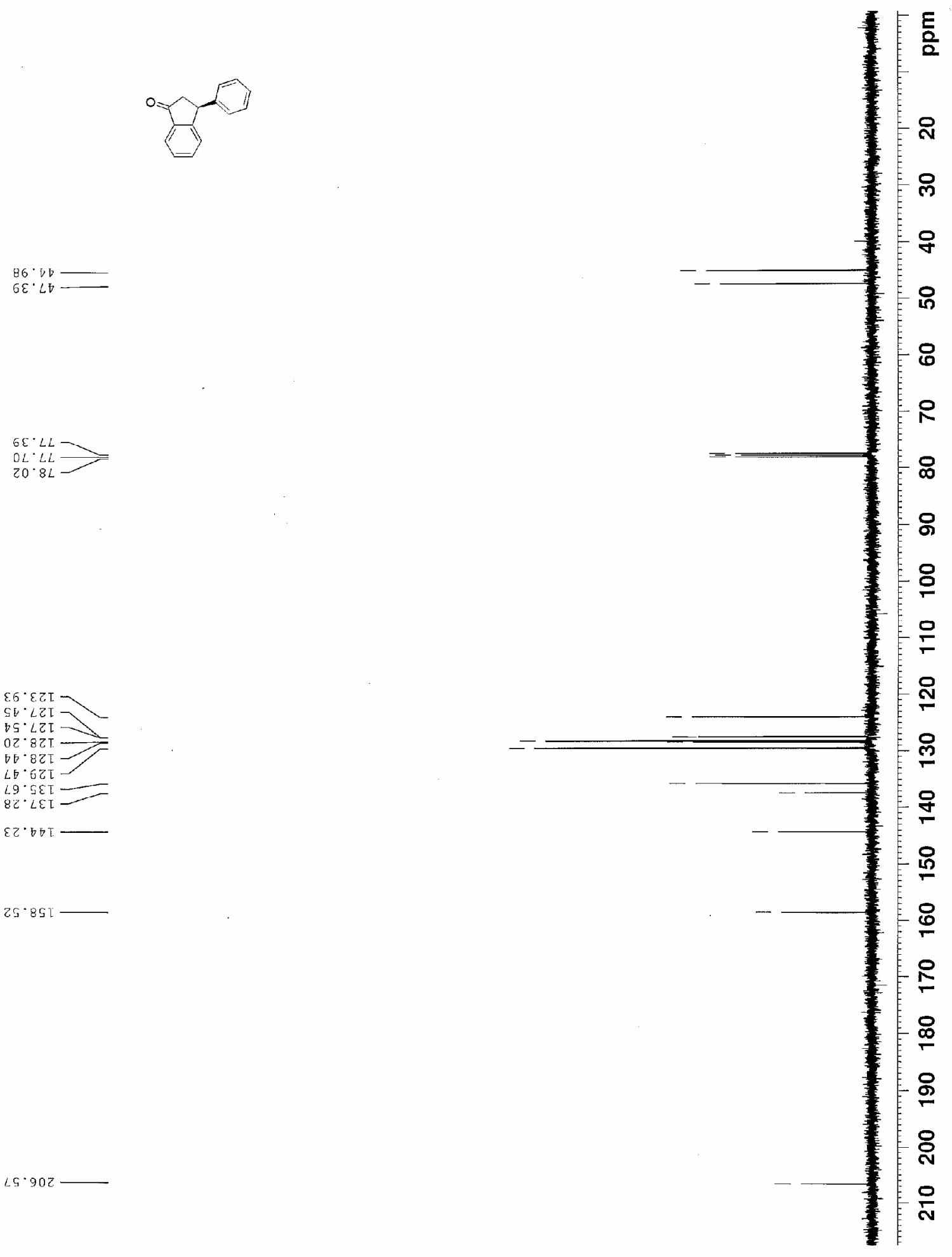

$15^{\circ} 902$

s.

$65^{\circ} \angle Z T$

be.8zT

$\angle \sigma^{\circ} \in Z T$

$\angle 9^{\circ} \mathrm{SET}-$

$25^{\circ} 851$ 

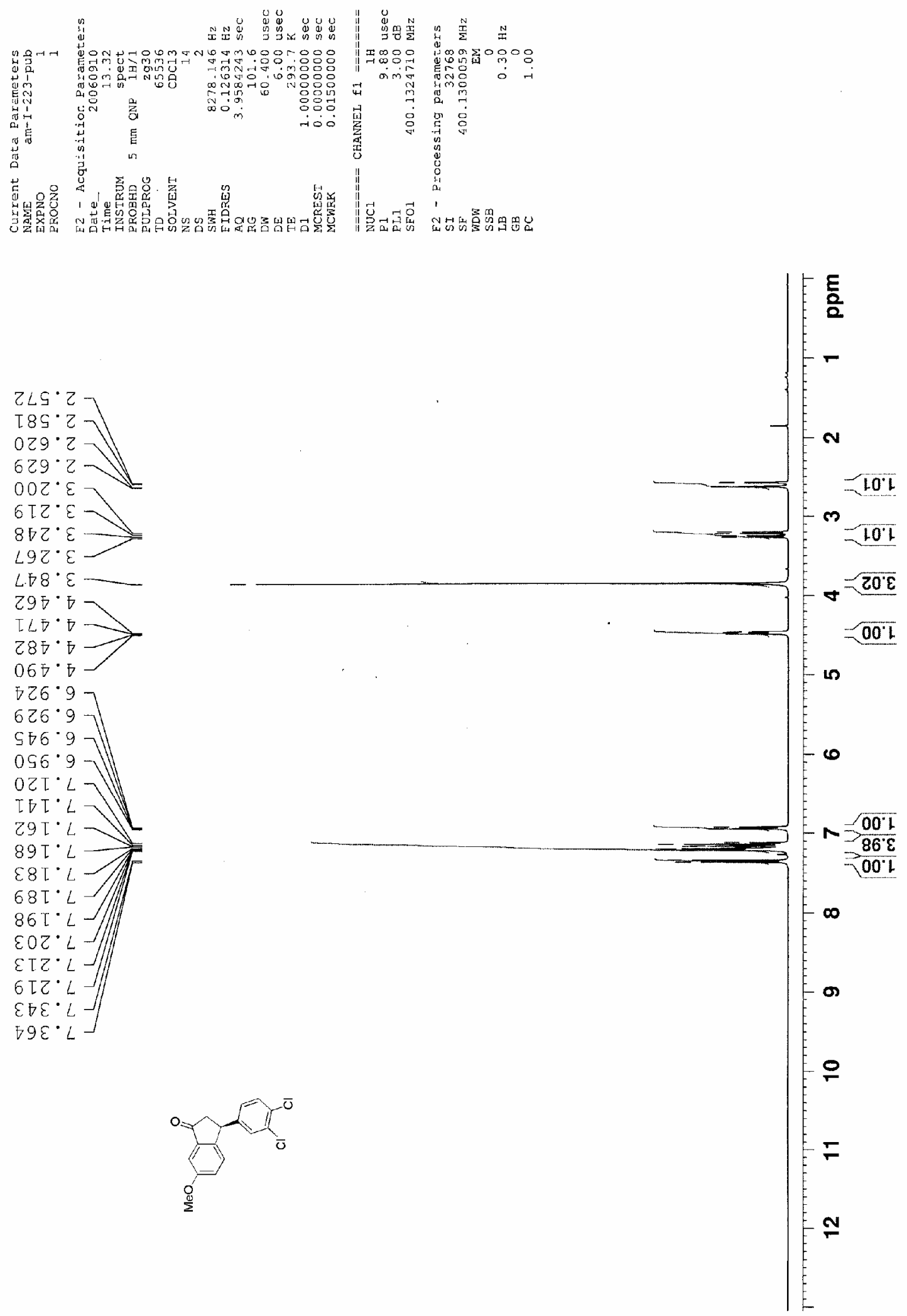

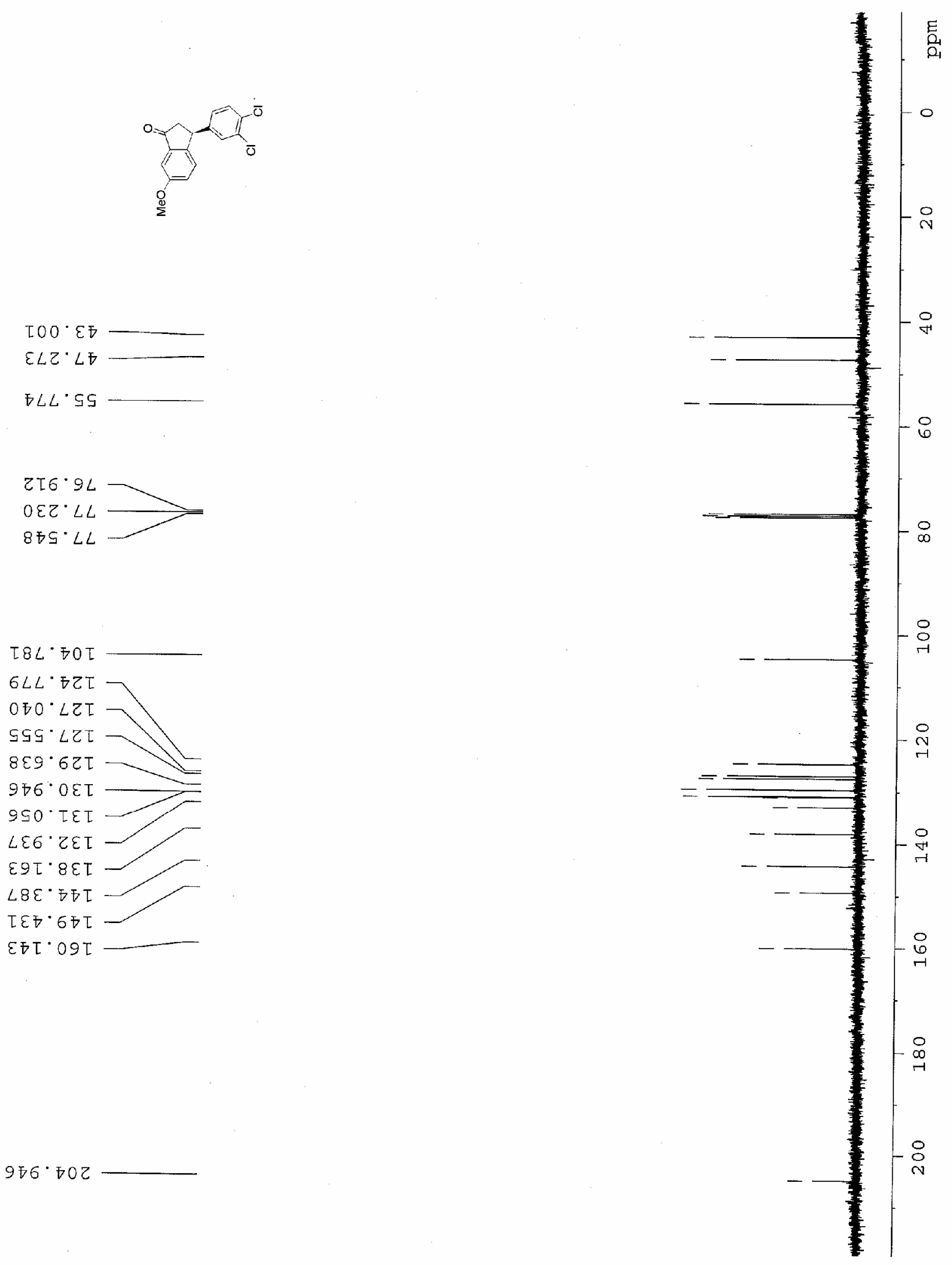

$906^{\circ} 702$ 

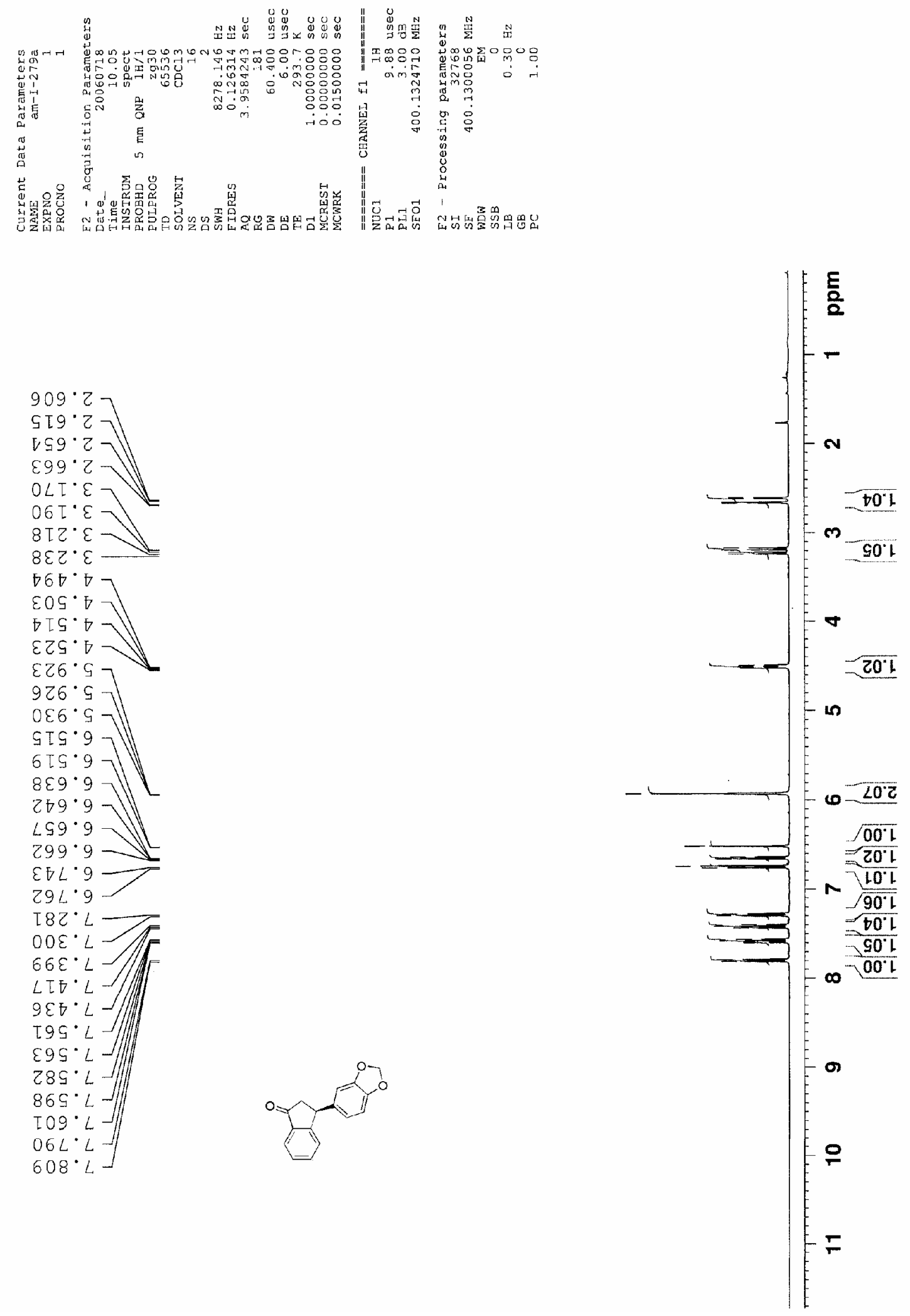

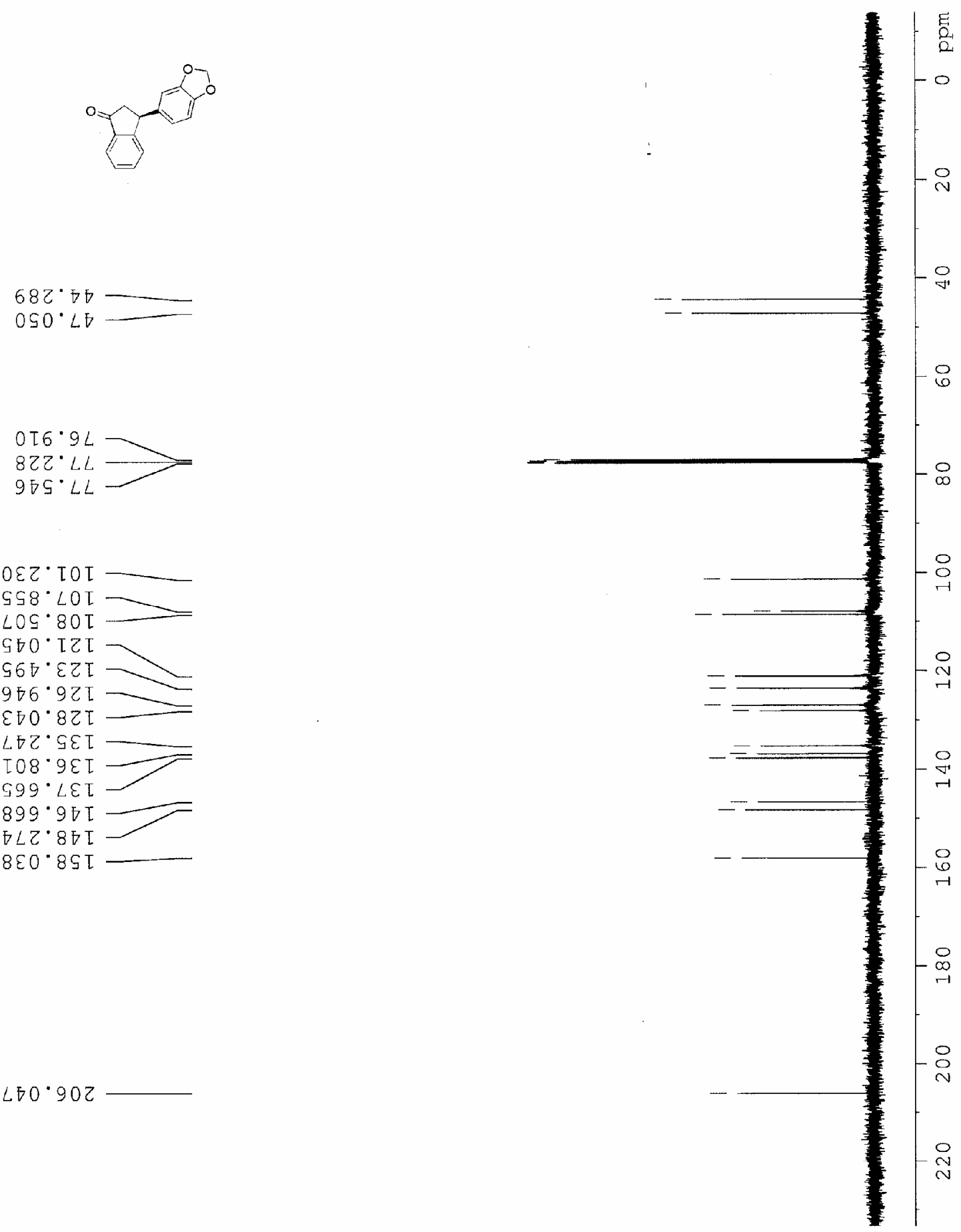

OEZ TOT

SS $8^{\circ} \mathrm{LOI} \longrightarrow$

$\mathrm{LOS}^{\circ} 80 \mathrm{~T}$

ร60. T己 I

$960^{\circ} \varepsilon Z T \longrightarrow$

$976.92 \mathrm{Z}$

$\varepsilon \mp 0 \cdot 82 T$

$\angle \boxminus Z \cdot \Im \varepsilon \tau$

โ08. $9 \varepsilon \tau$

$599^{\circ} \angle E L$

$899^{\circ} 9 \mathrm{VI}$

$6 L Z^{\circ} 8 \mathrm{FL}$

$8 E 0 \cdot 8 \mathrm{I}$

$\angle 80 \cdot 902$

s 

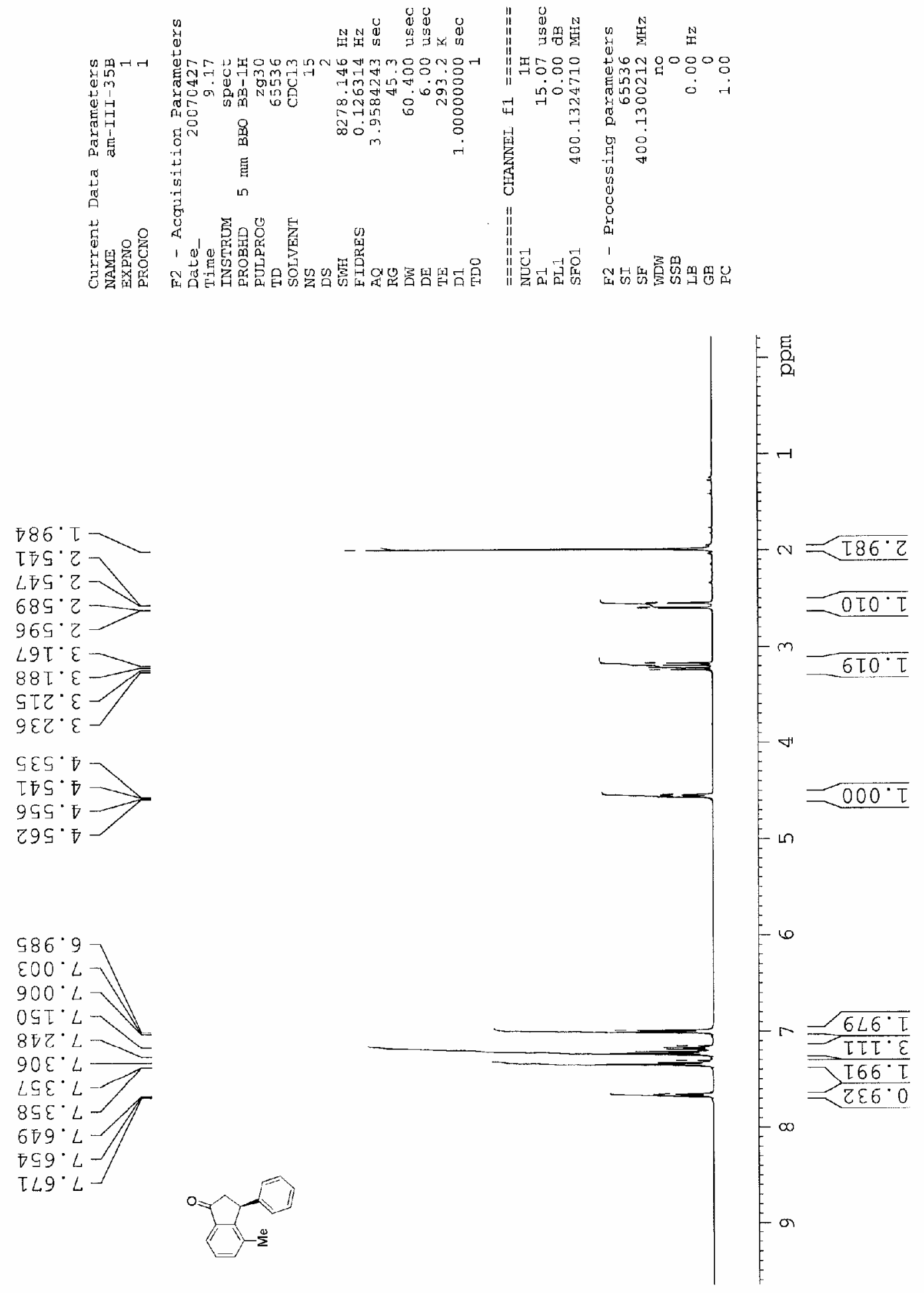

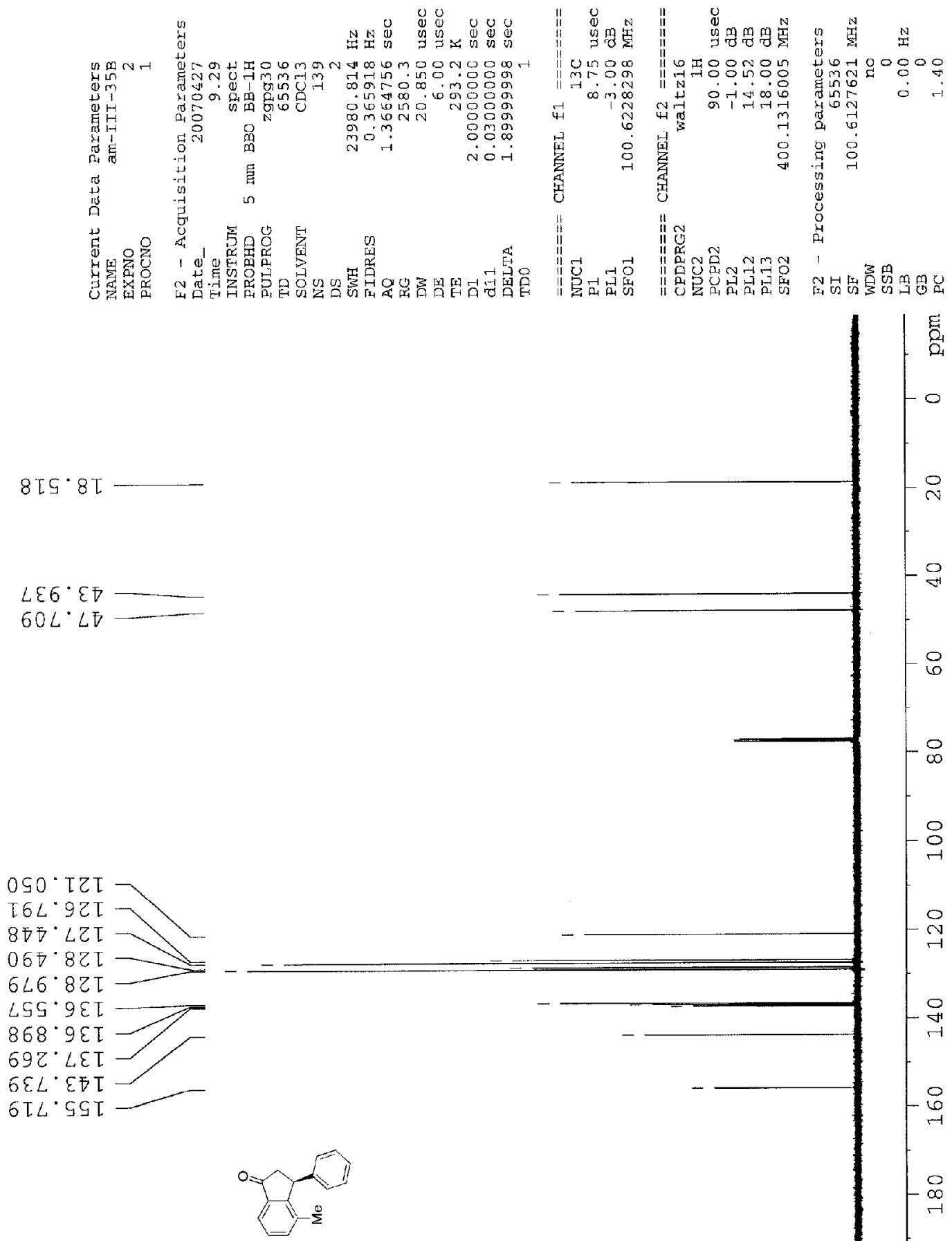

$67 L \cdot 902$
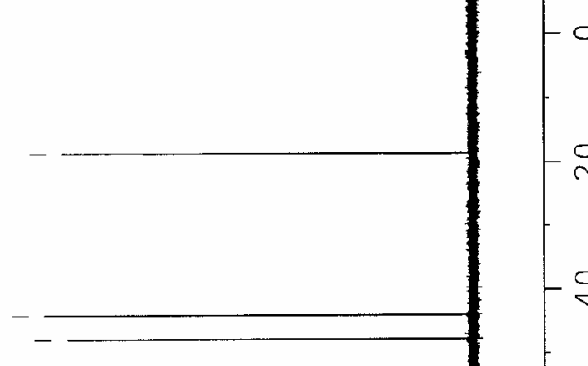

$\stackrel{i}{N}$

- 국

$-8$

-

$\infty$

욱

옥

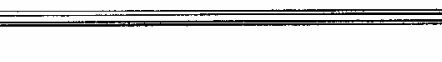

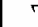

-

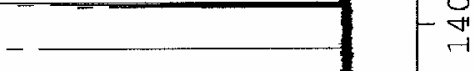

$\longrightarrow$

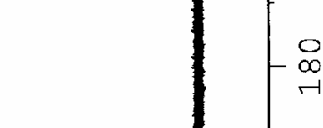

$-\longrightarrow$ 

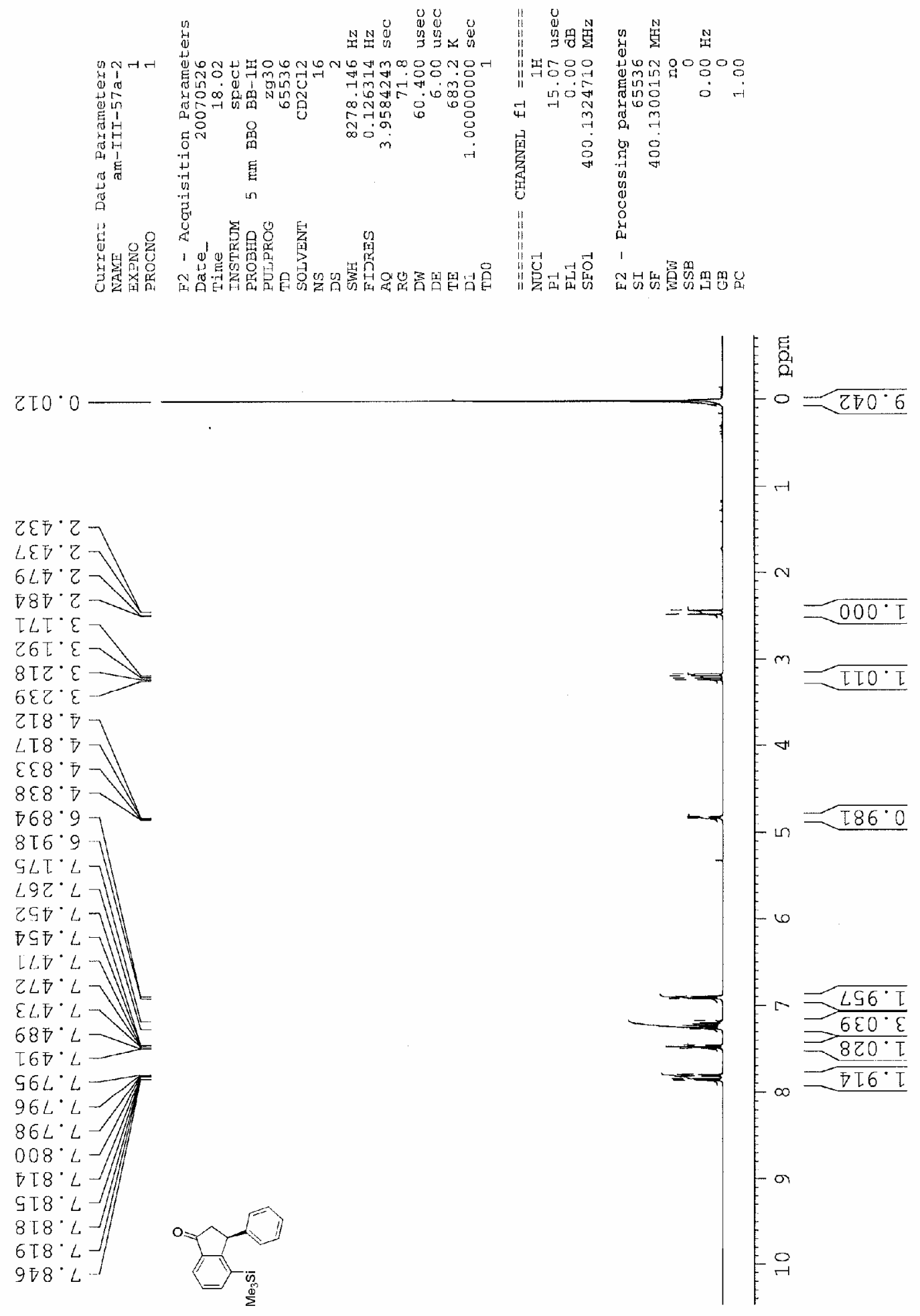


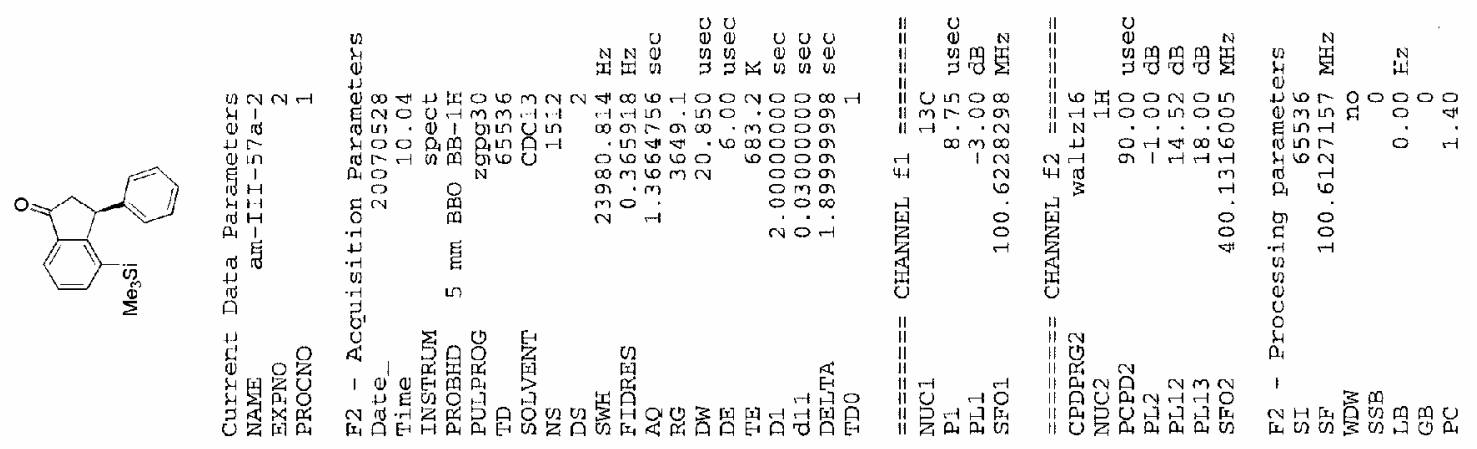

$6 z \varepsilon^{\circ} 0^{-}$
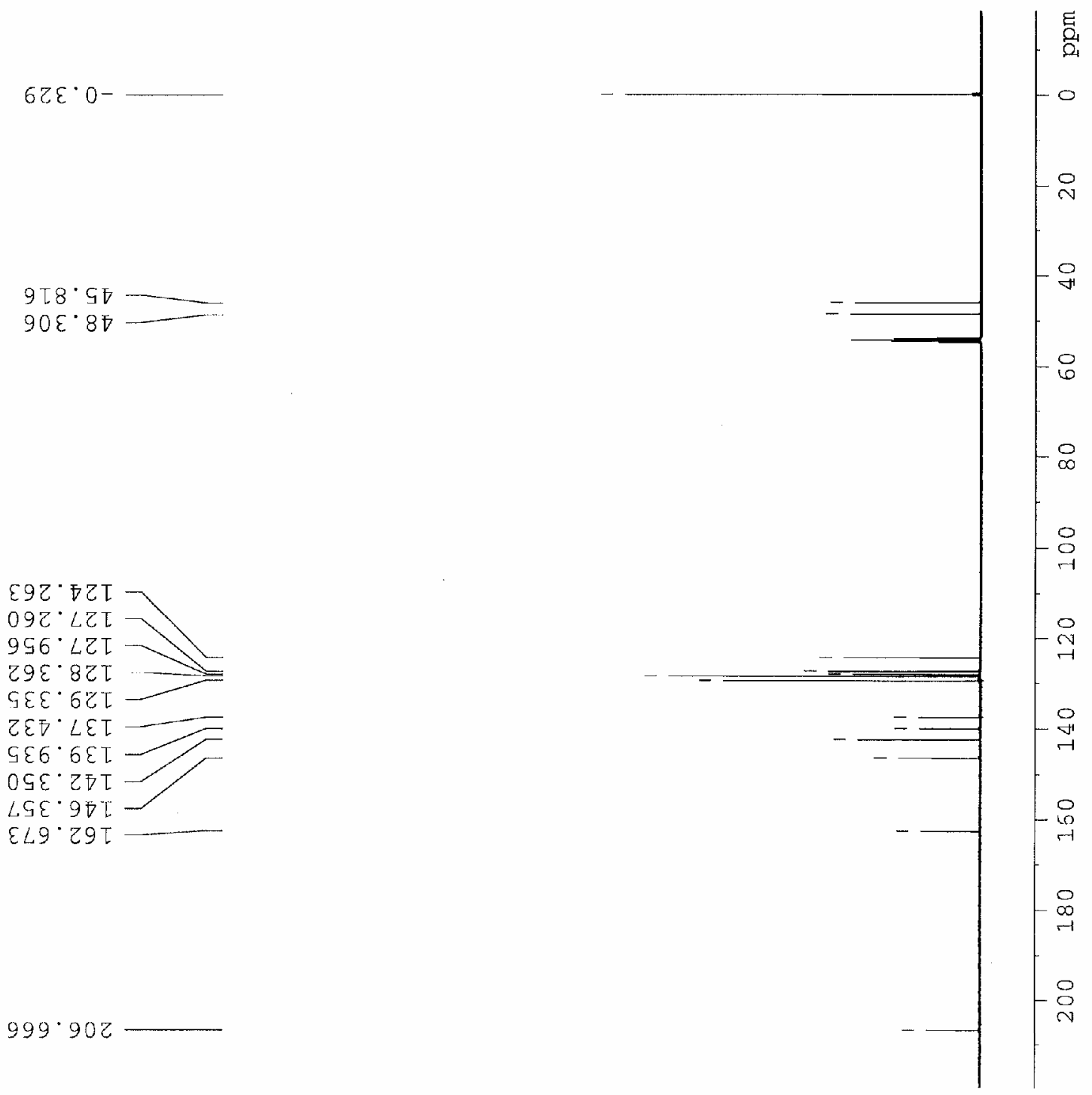

$999 \cdot 902$ 

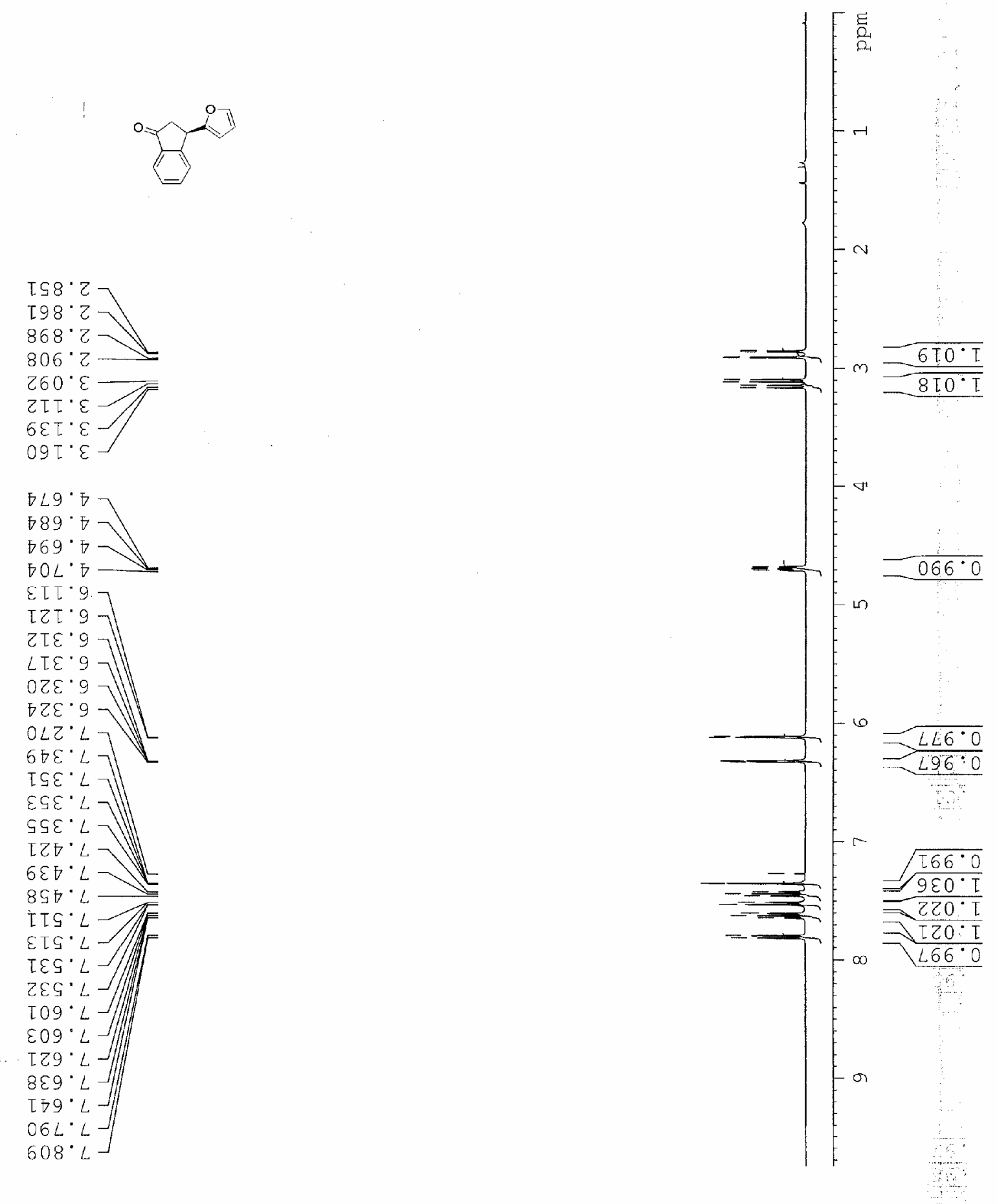

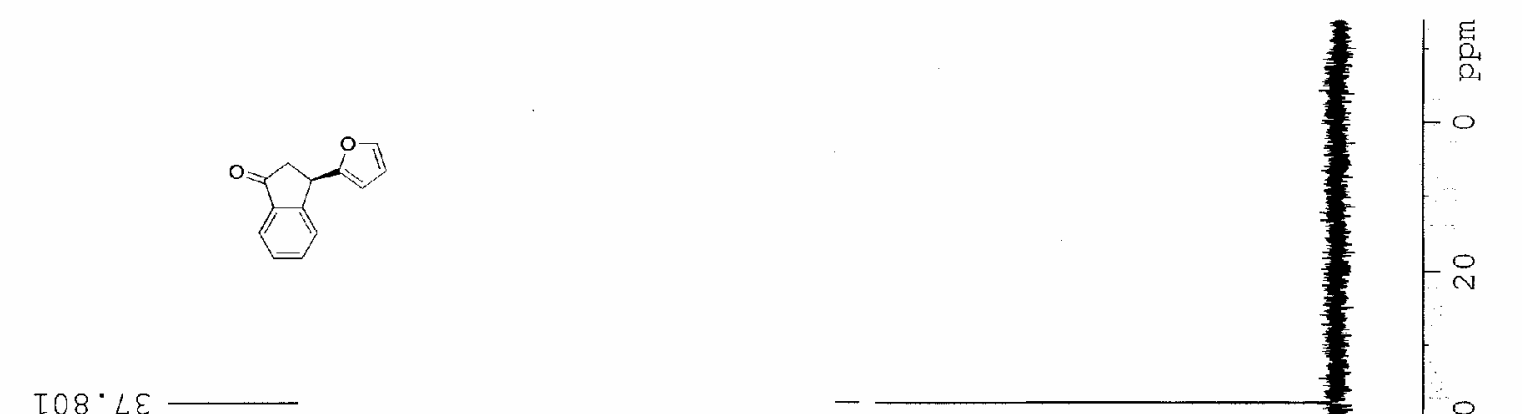

TO8. LE

$526 \cdot 27$

$606 \cdot 94$

$\angle 2 Z \cdot \angle L$

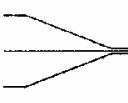

$\angle L 6^{\circ} \mathrm{COL}$

$8 Z 8^{\circ} \varepsilon ट \tau$

ZSL.9ZT

TEF.8ZT

\ST. $5 E T$

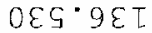

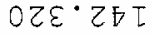

$\varepsilon 9 \varepsilon^{\circ}$ 乙ष

$928^{\circ} \nabla S I$

$\mathrm{L62} \cdot \mathrm{GSI}$

$60 \mathrm{~T} \cdot \mathrm{GOZ}$

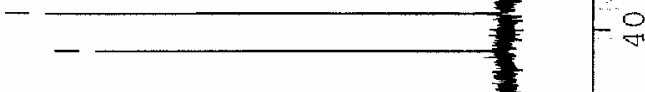

$-8$
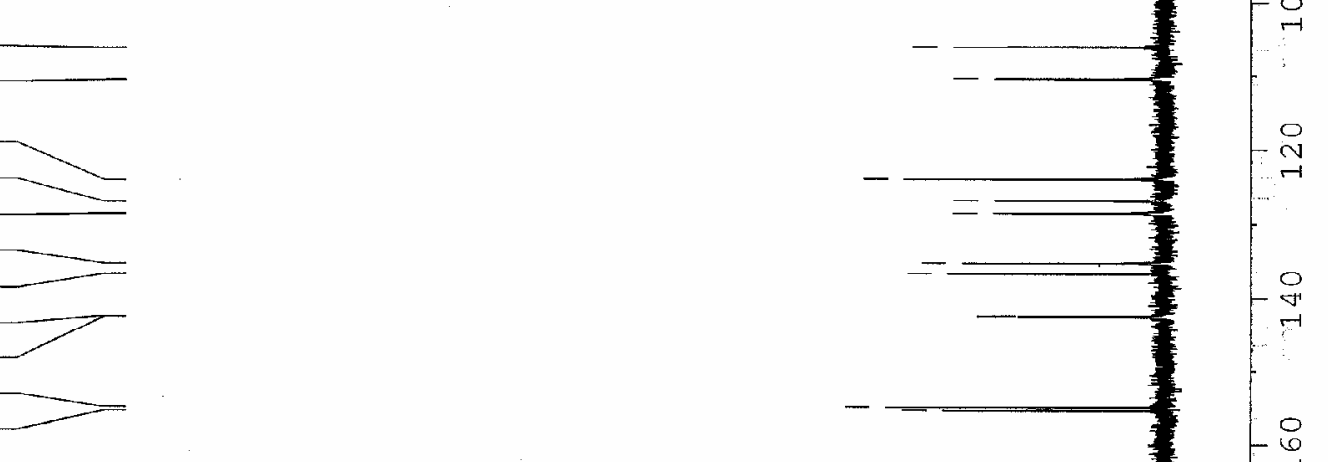

$-8$

$-\infty$
$-\infty$

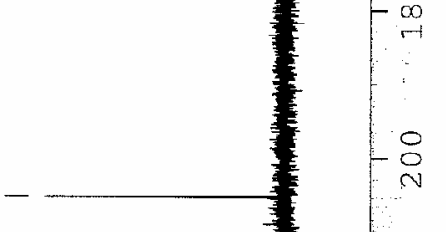



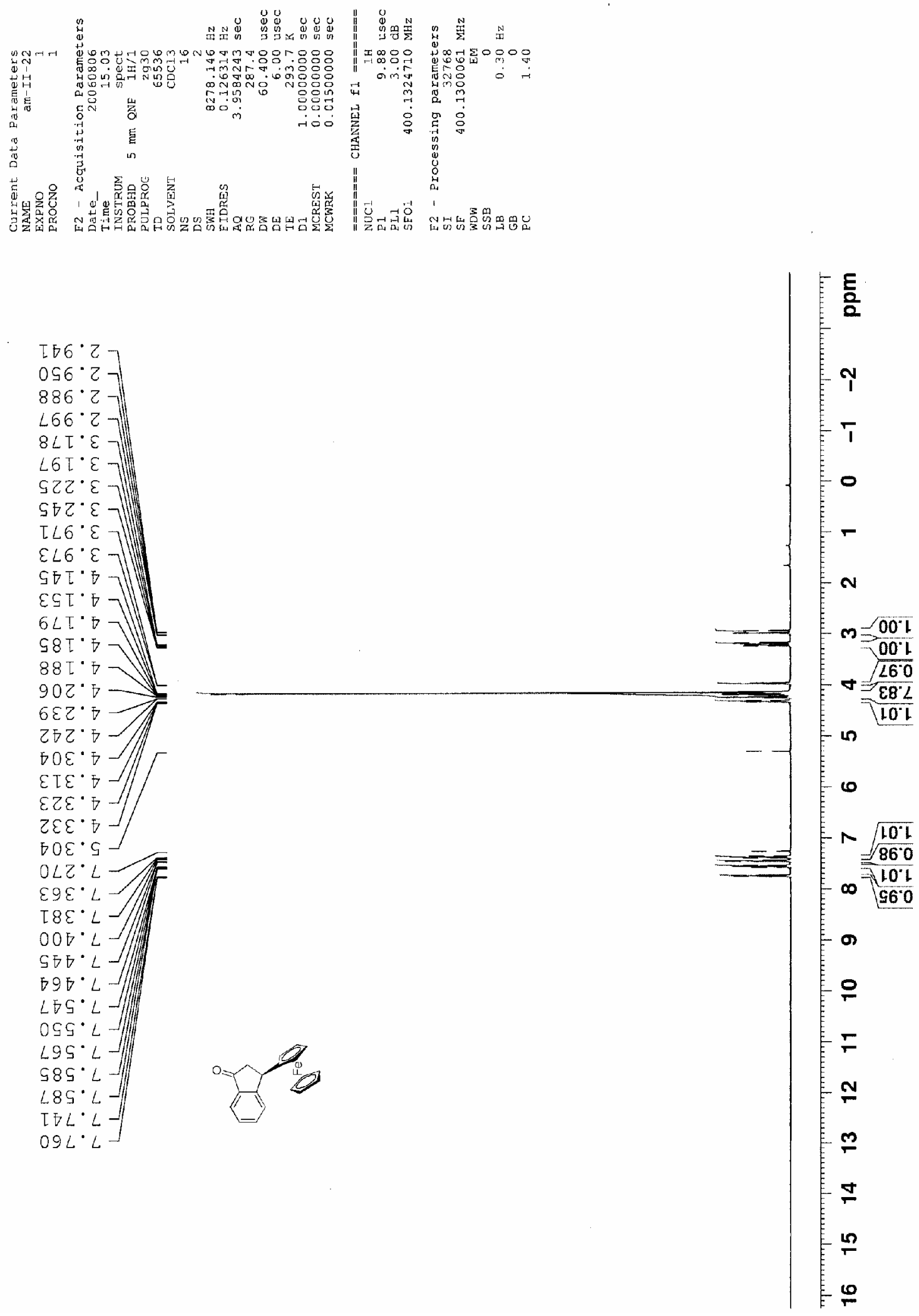

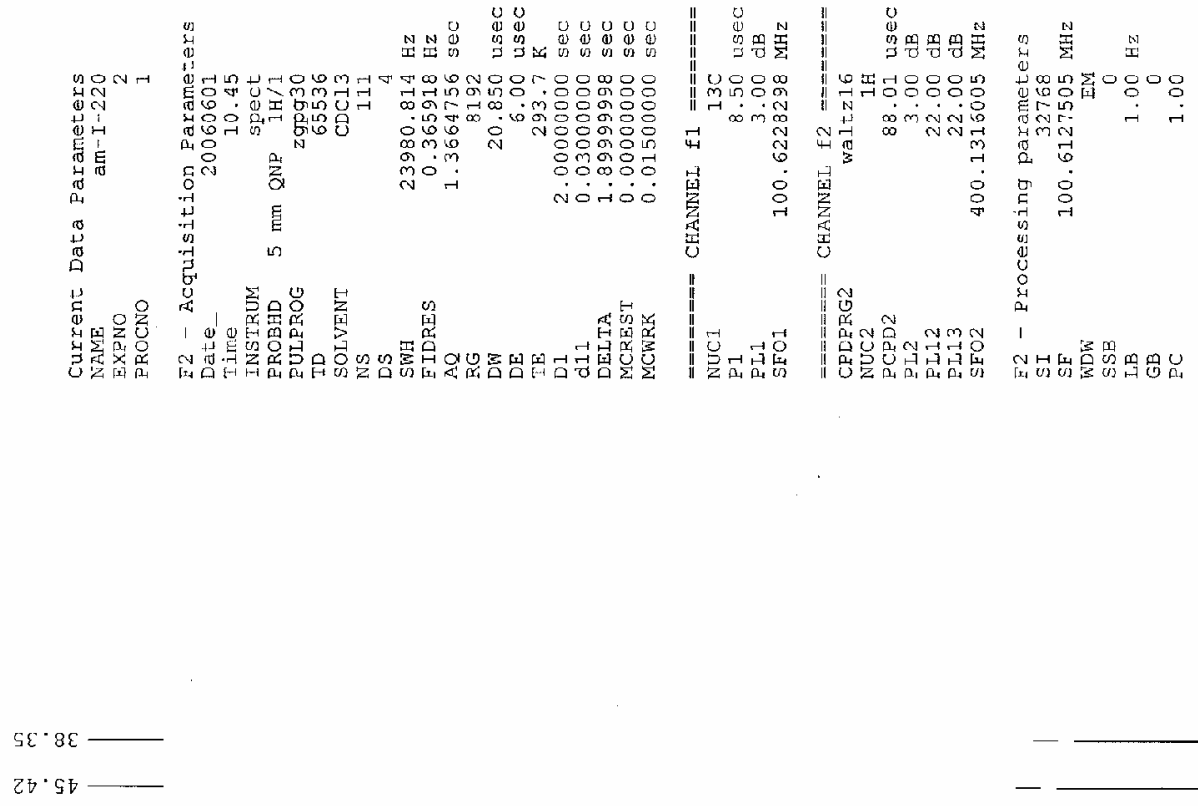

$99^{\circ} \varepsilon Z \mathrm{~T}$

S7. $92 \mathrm{ZL}$

L6. $\mathrm{ZZT}$

86. $\operatorname{t\varepsilon }=$
ST.9E[ $=$

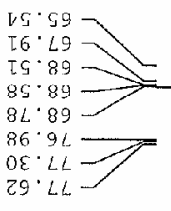

$10 \cdot 16$

I. gel-

$8 I \cdot 85 \tau$

$z \varepsilon \cdot 90 z$

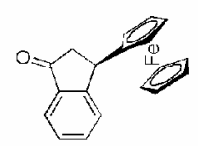

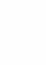



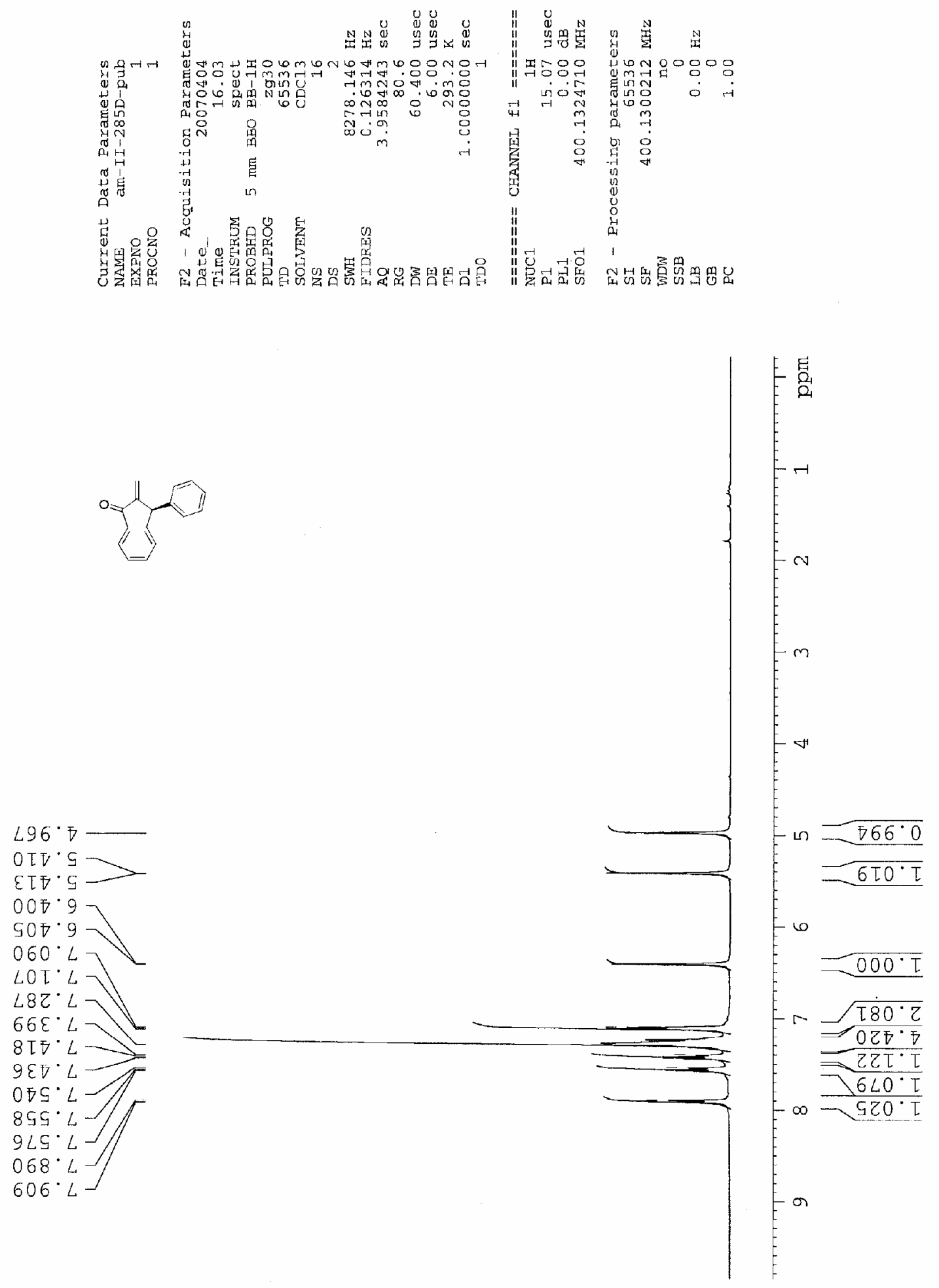

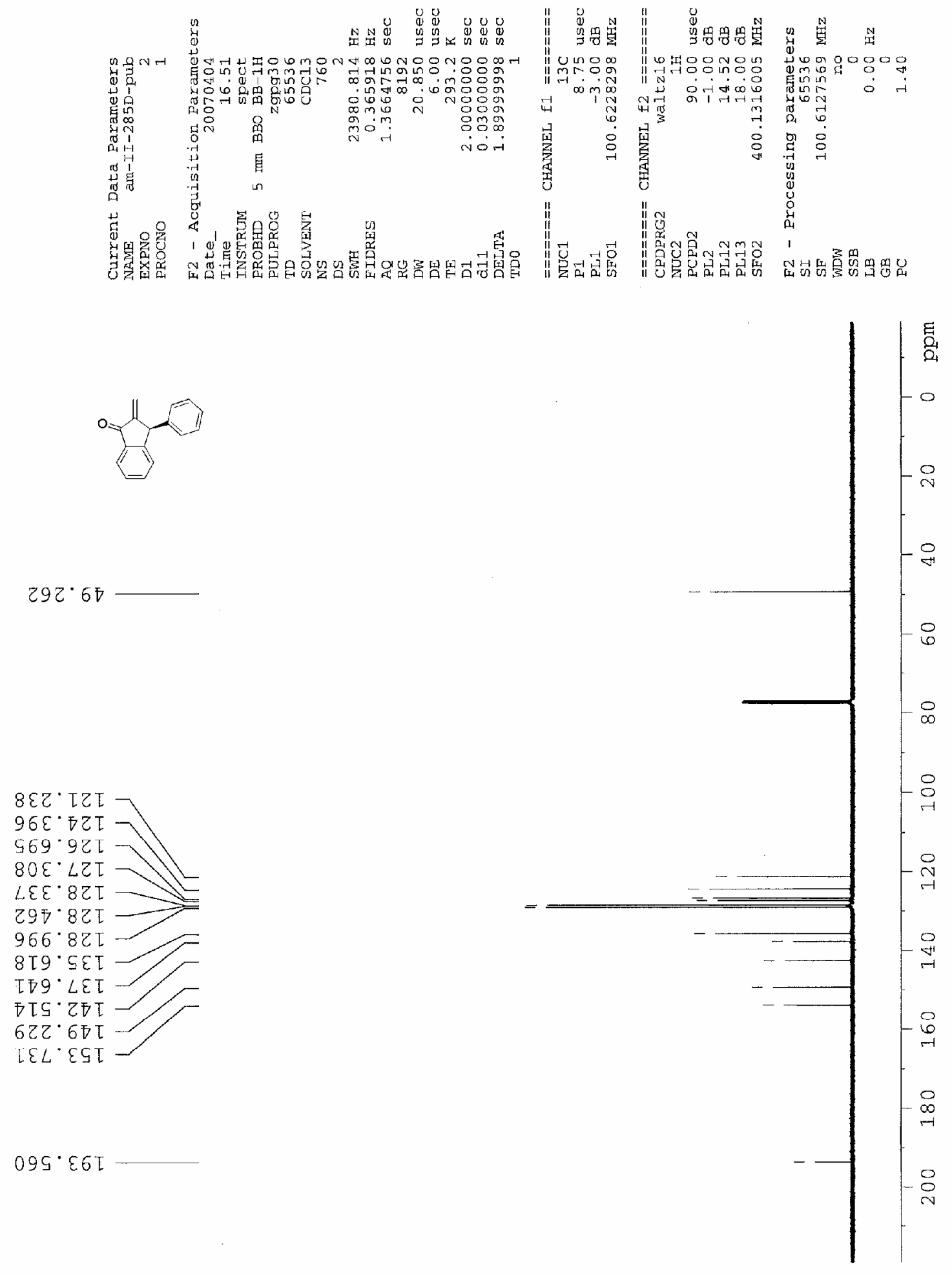

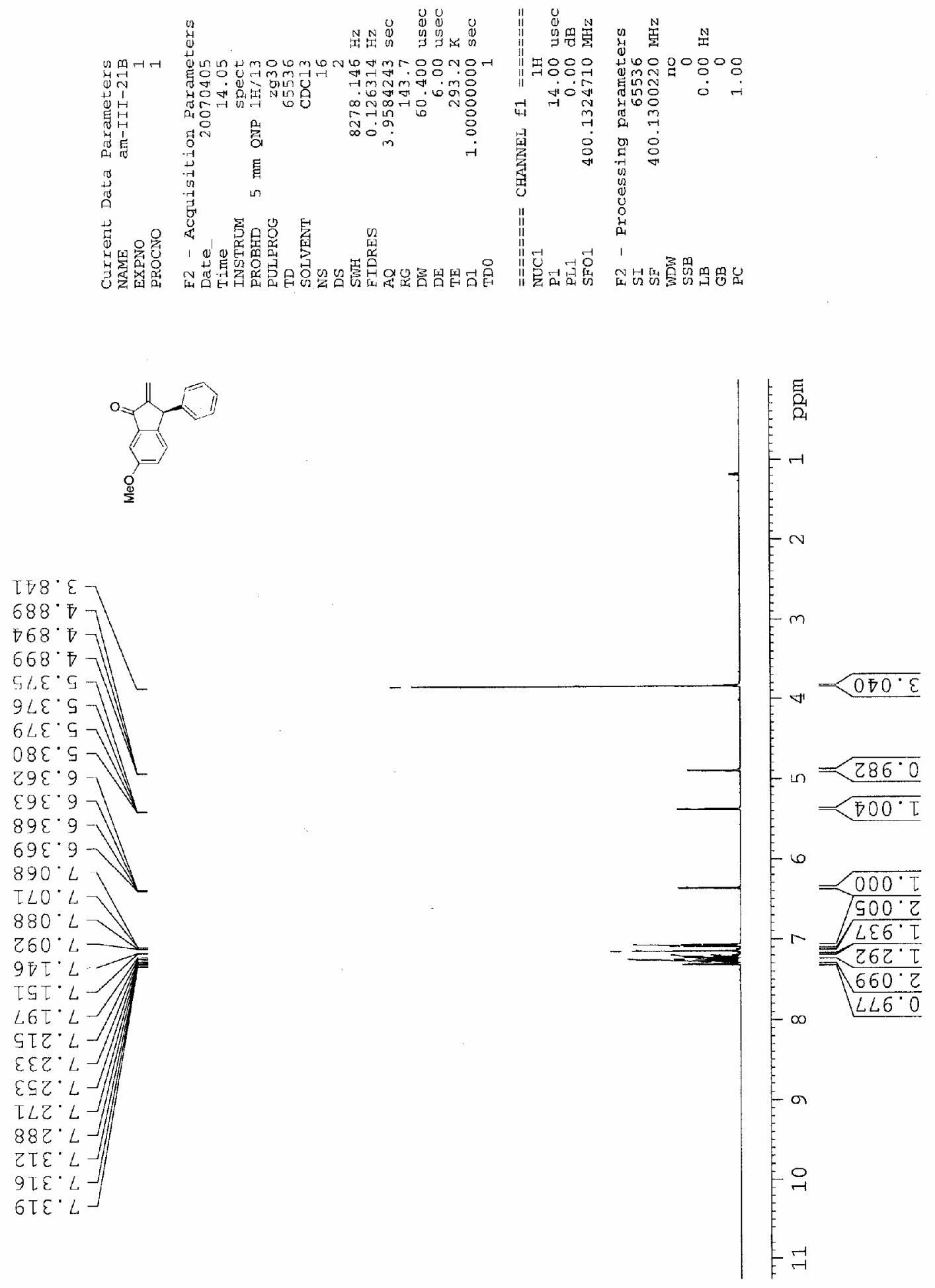

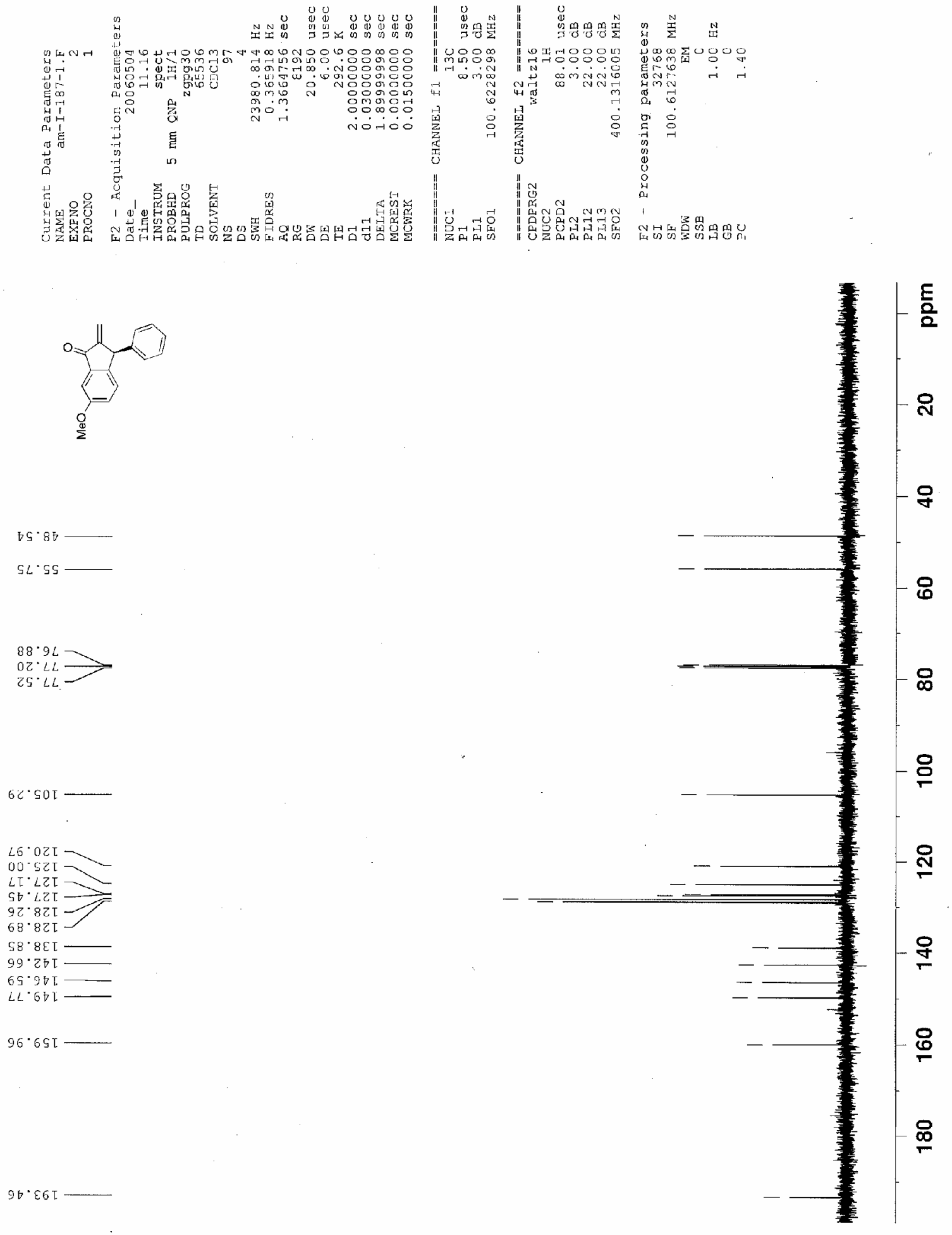

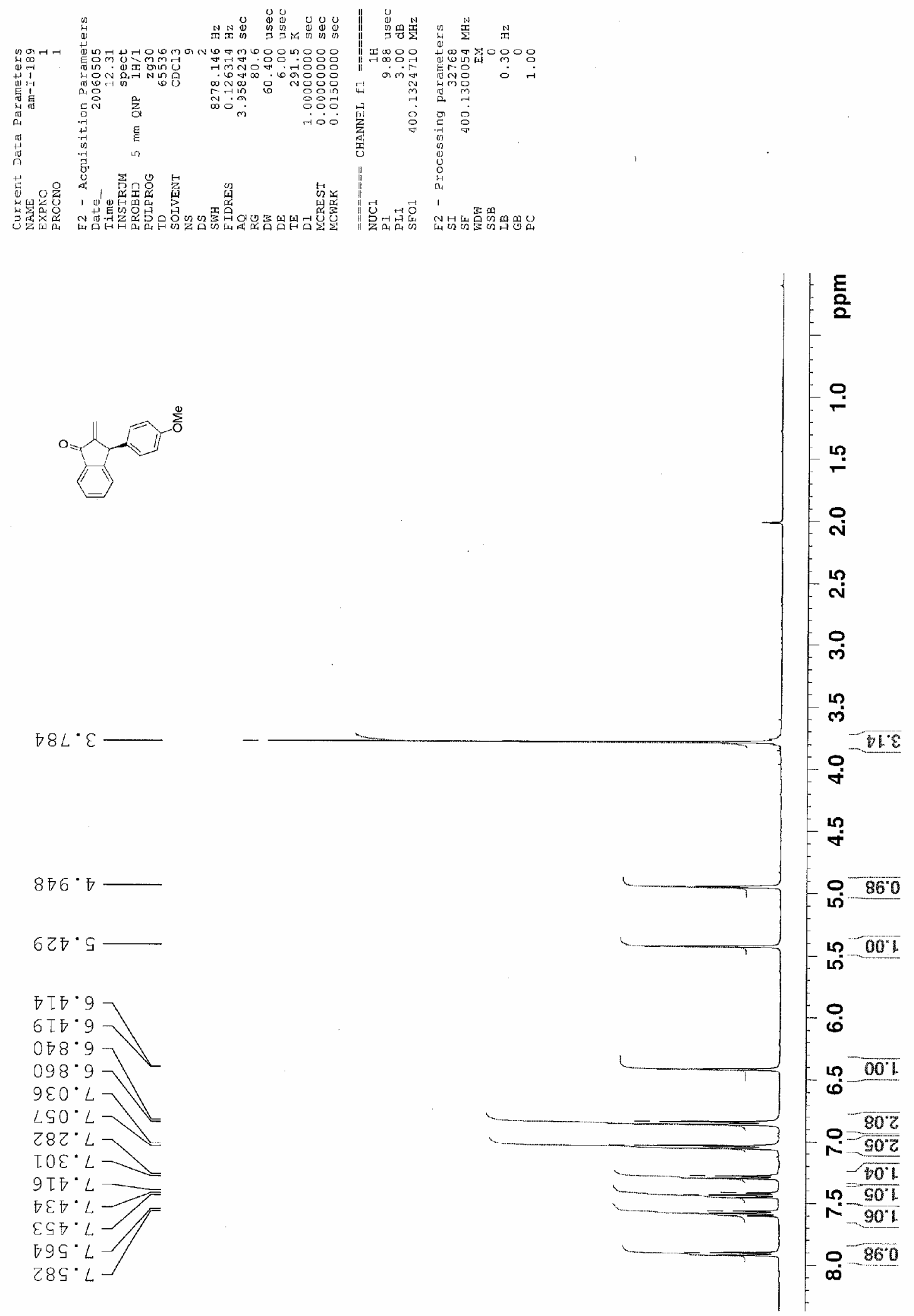

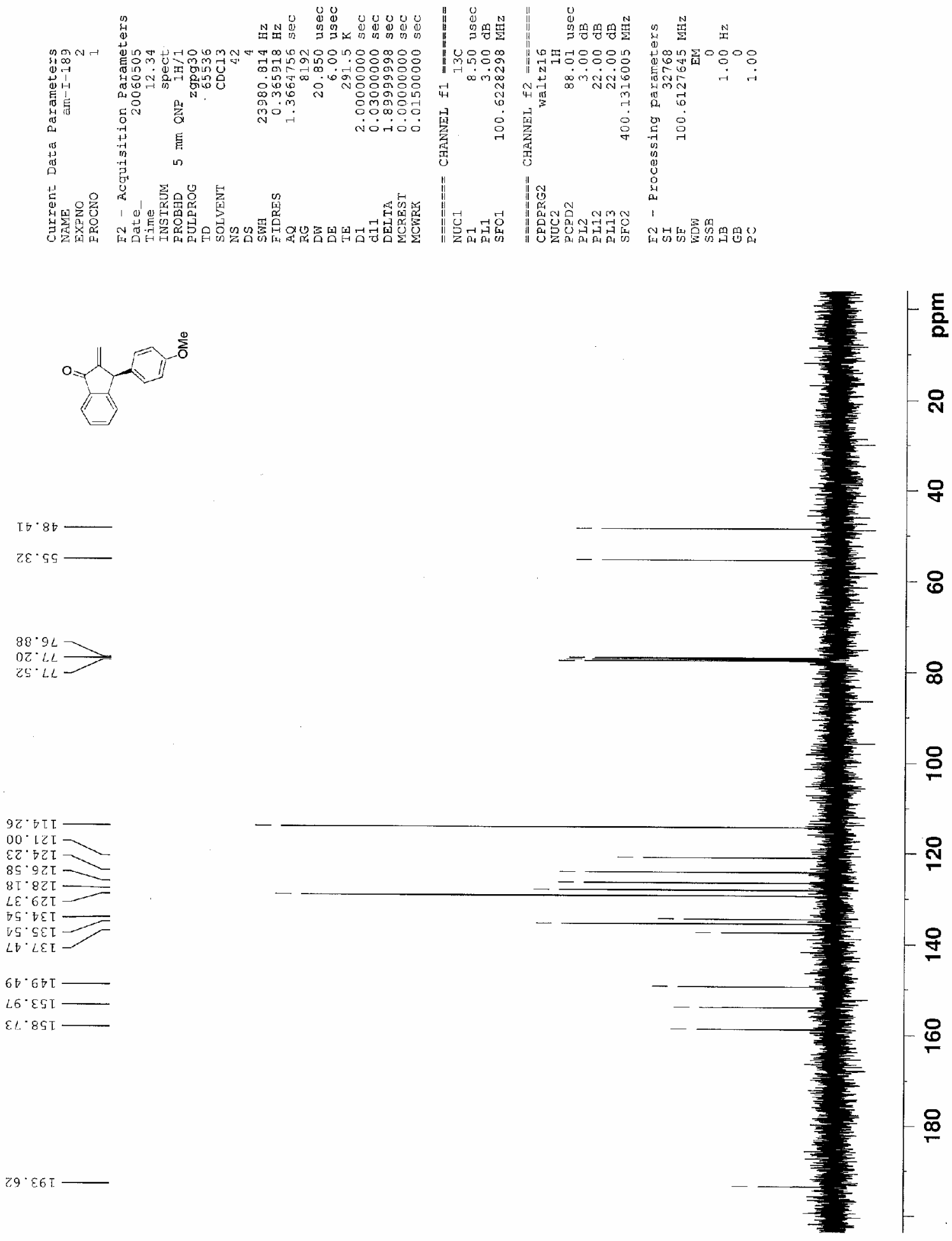

$9 C^{\circ}$ ET L

$00^{\circ} \operatorname{LEL} \longrightarrow$

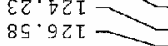

$8 \mathrm{I} \cdot 8 \mathrm{IL} \longrightarrow$

$\angle \varepsilon^{\circ} 6 z \mathrm{~T}$

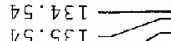

LV.LEL

$6 \nabla^{\circ} 65 \mathrm{~T}$

$\angle 6^{\circ} \varepsilon \tau_{\mathrm{J}}$

$\varepsilon t^{\circ} \cdot 8 \mathrm{TT}$

$29 \cdot 86 I-$ 

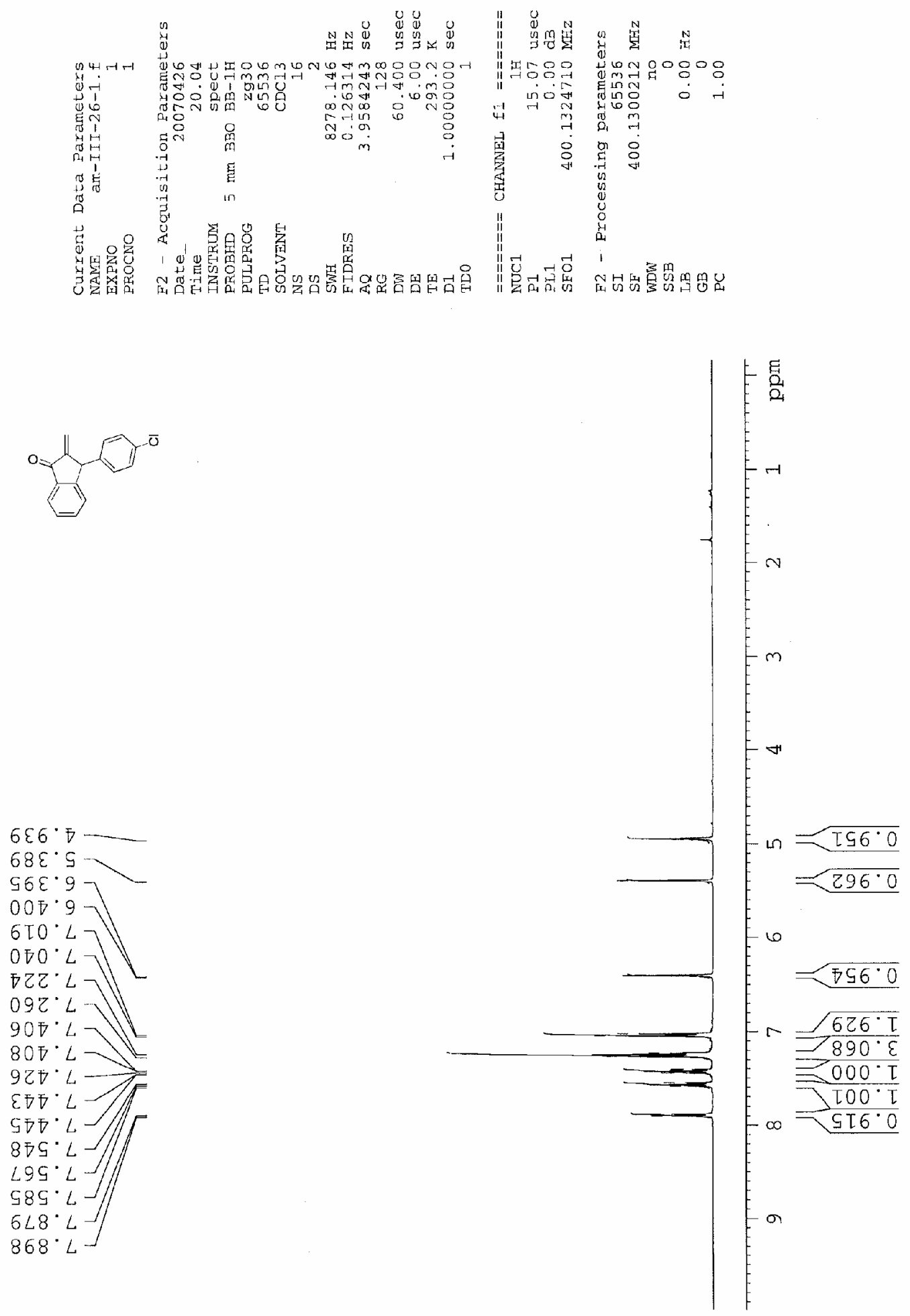


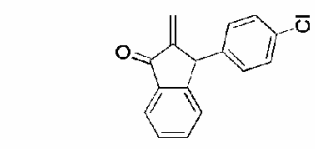

โ万9・8も
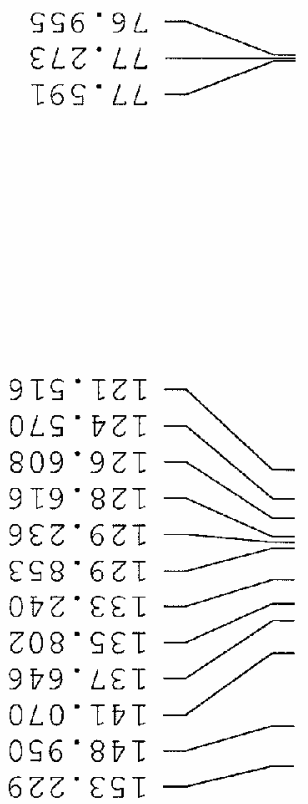

$\left[S Z^{*} \varepsilon 6 \tau\right.$

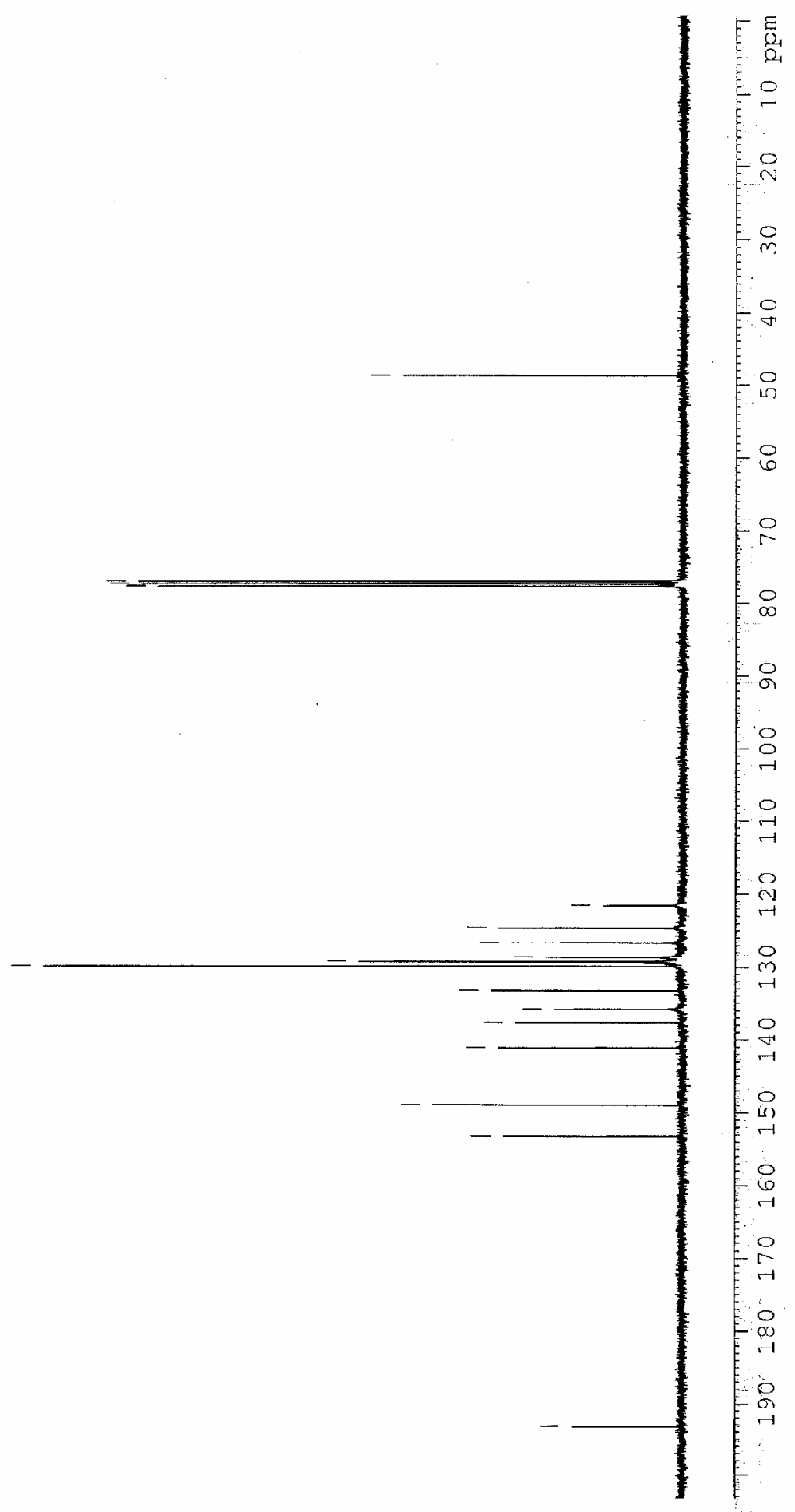



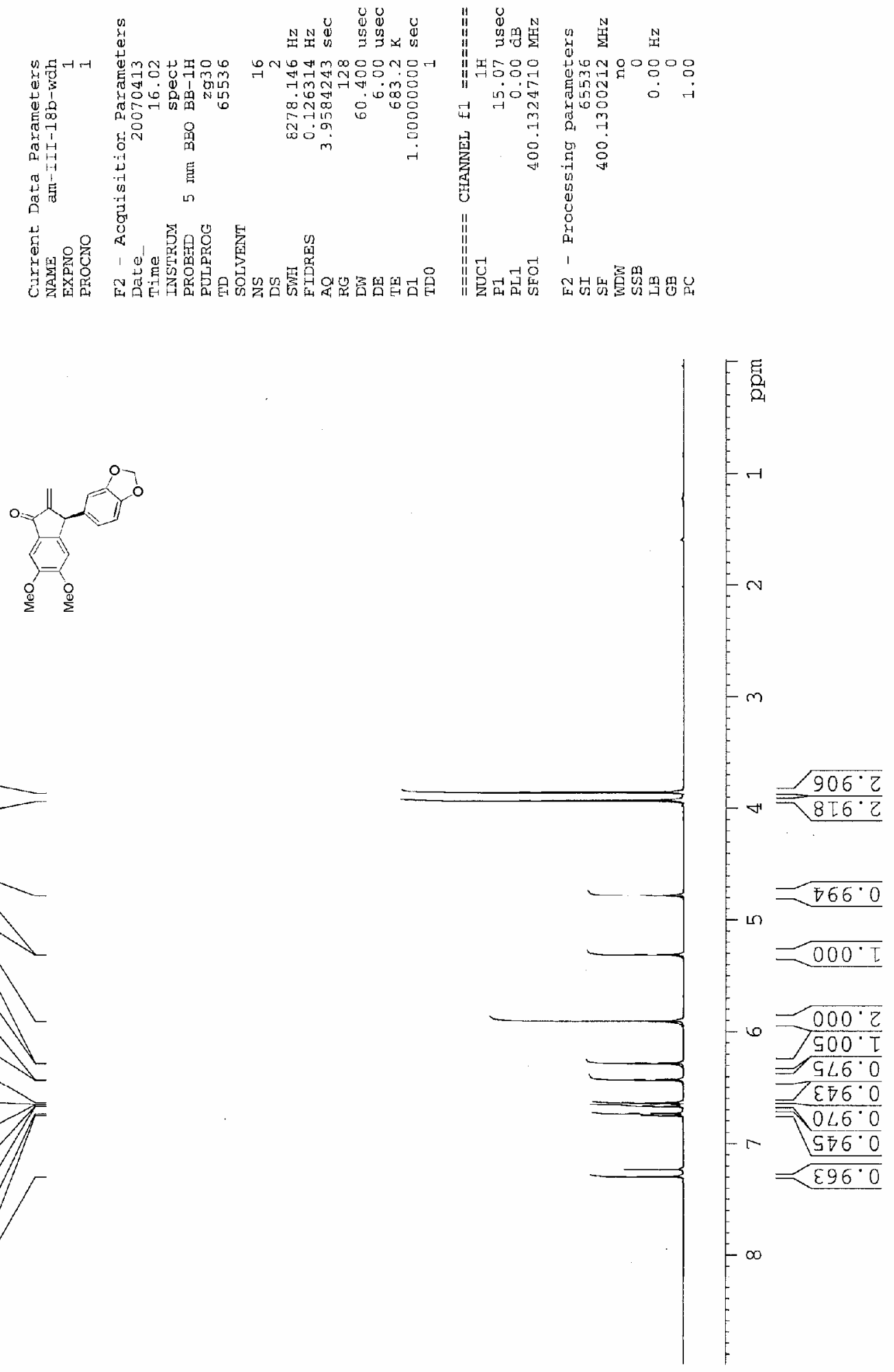

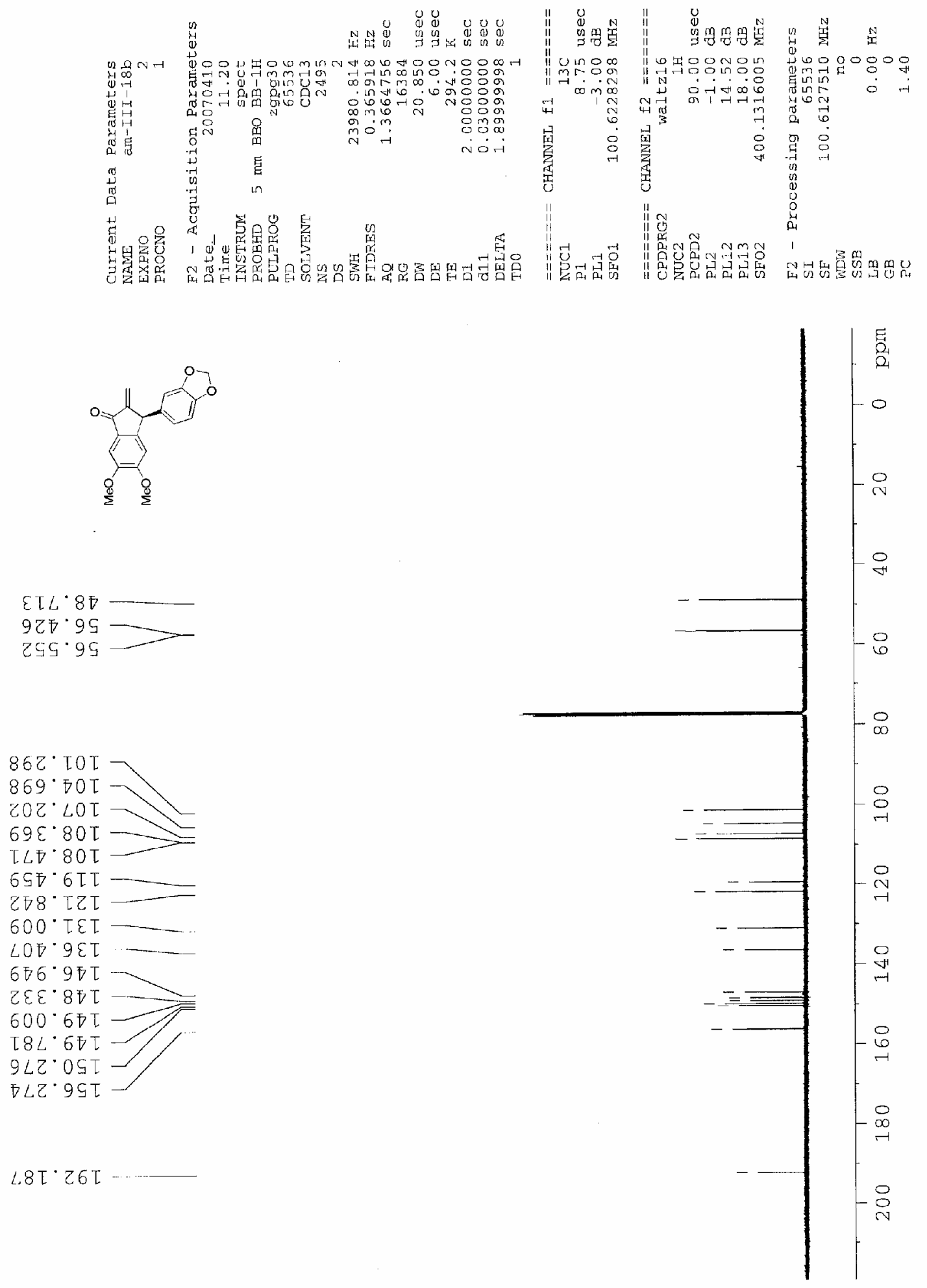

$28 T \cdot 26 \tau$ 

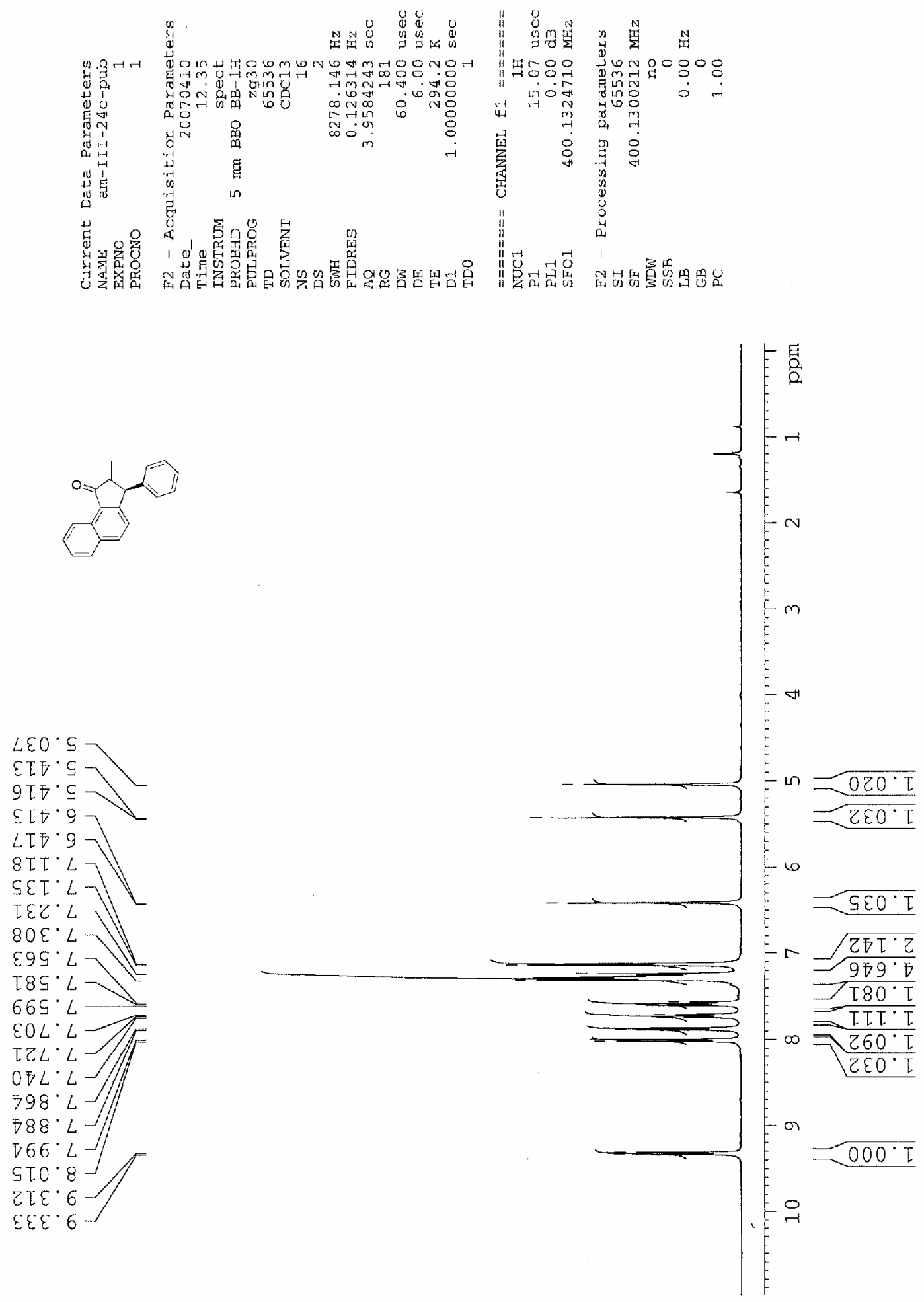

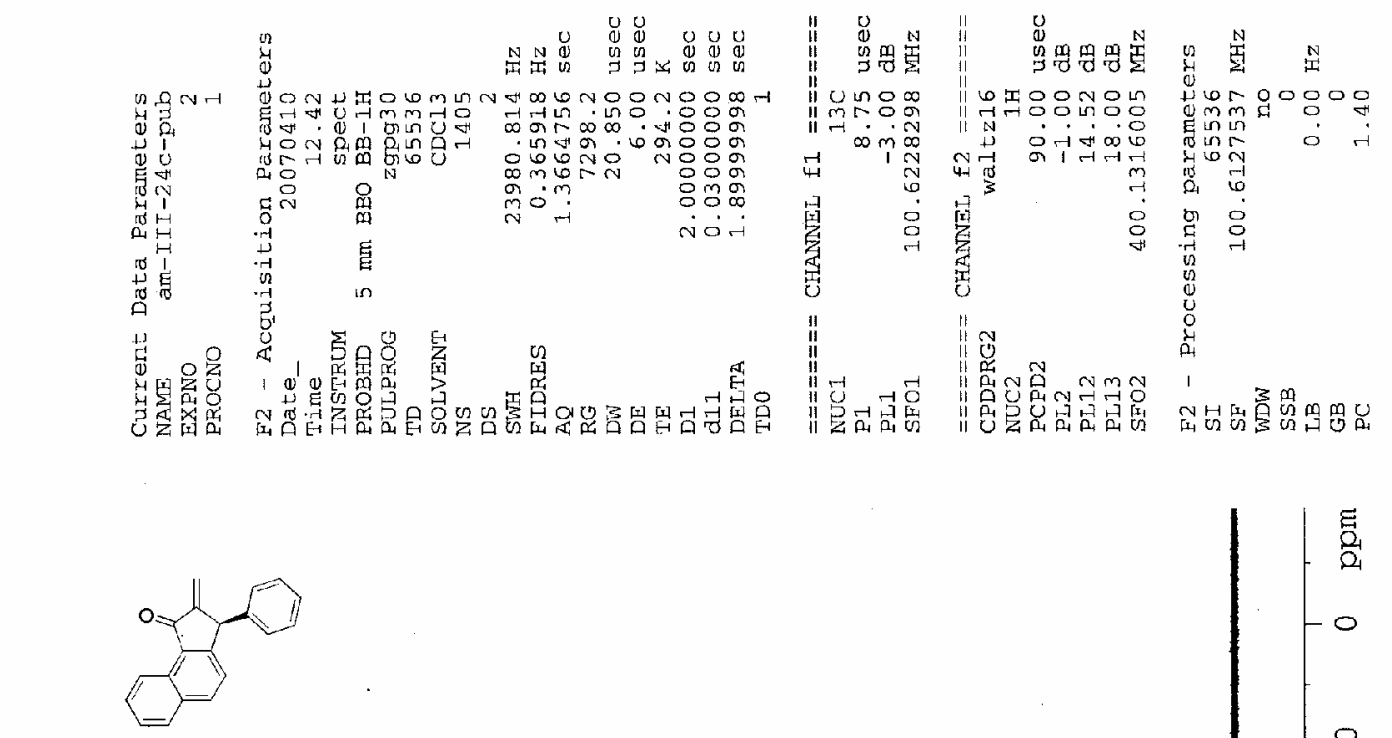

$\varpi \varsigma \varepsilon \cdot 6 \downarrow$
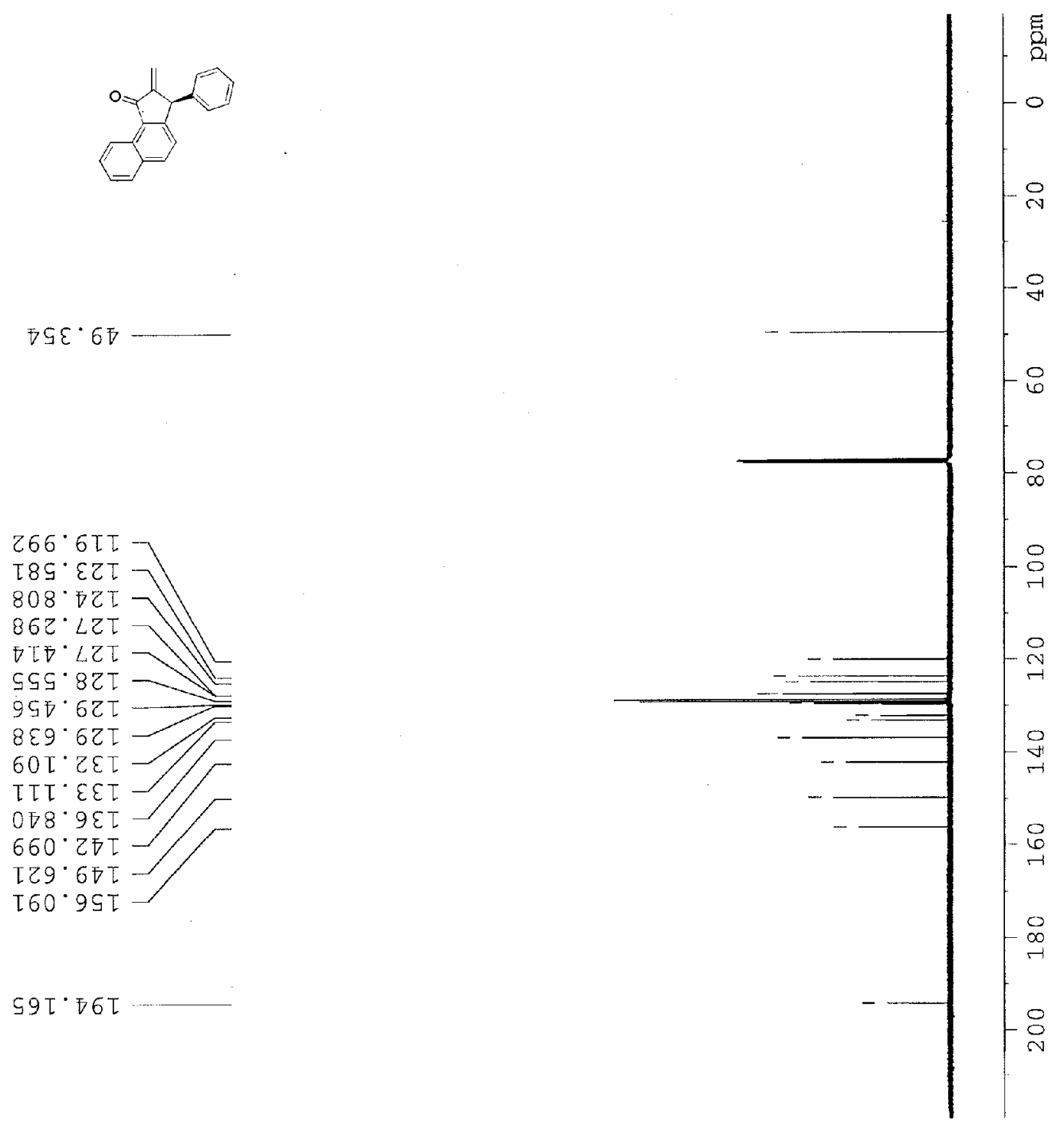


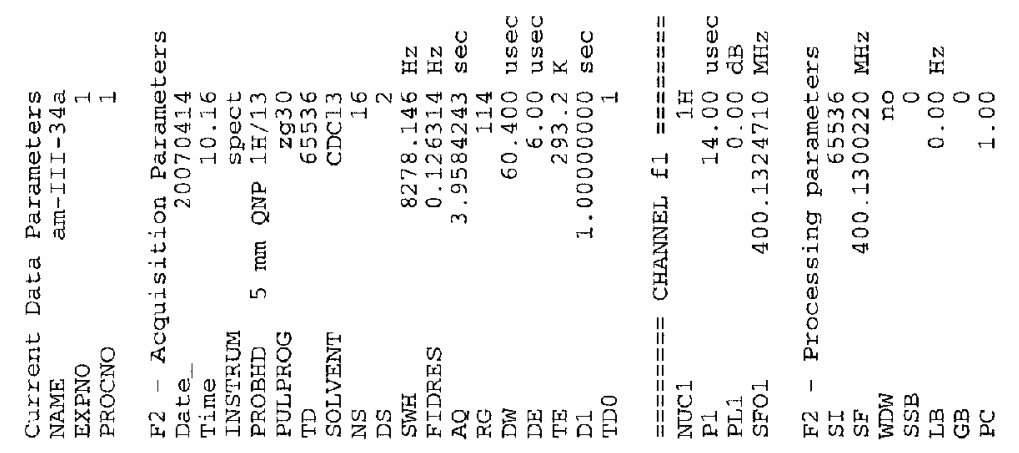

$996^{\circ} \tau$
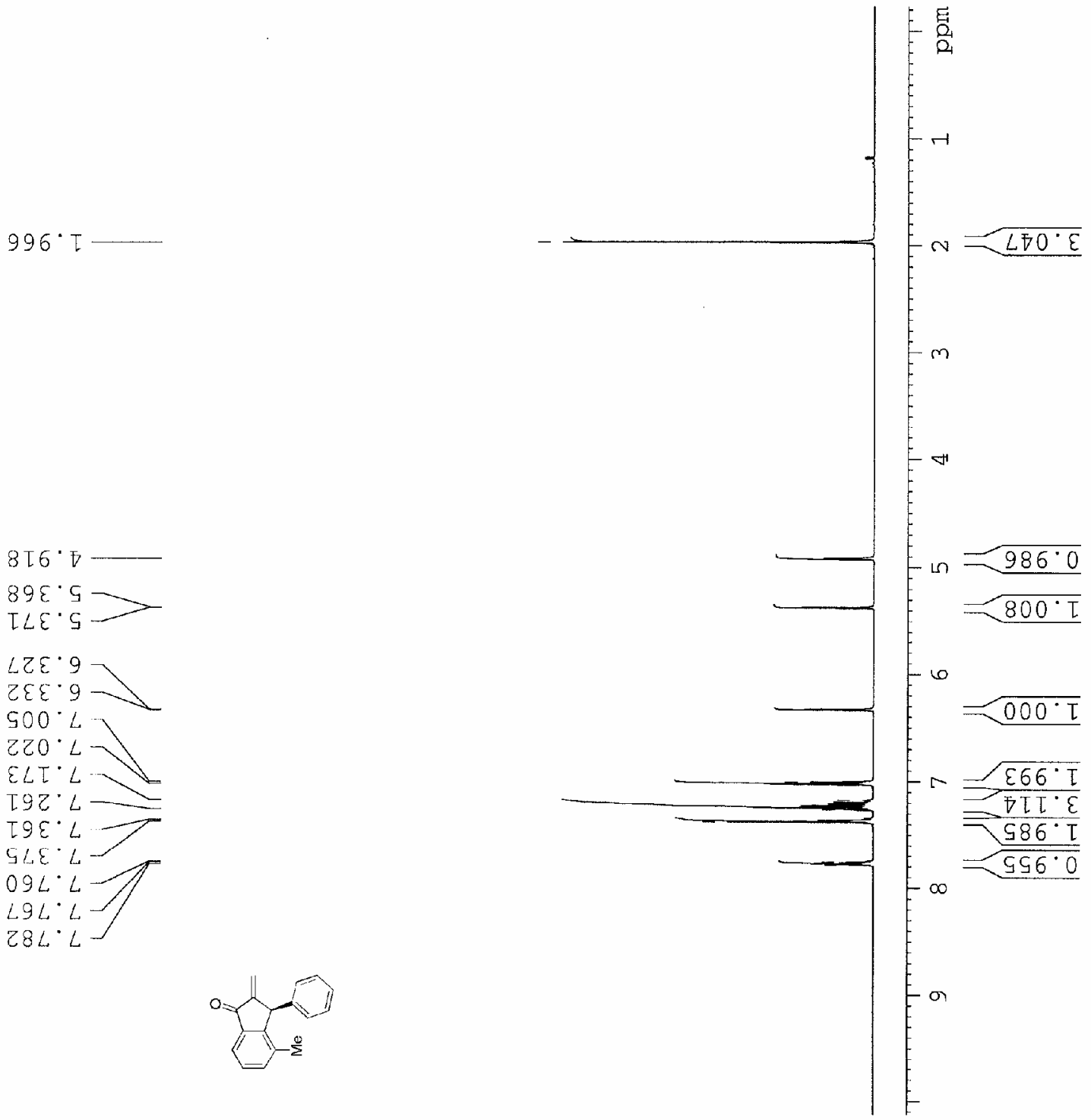

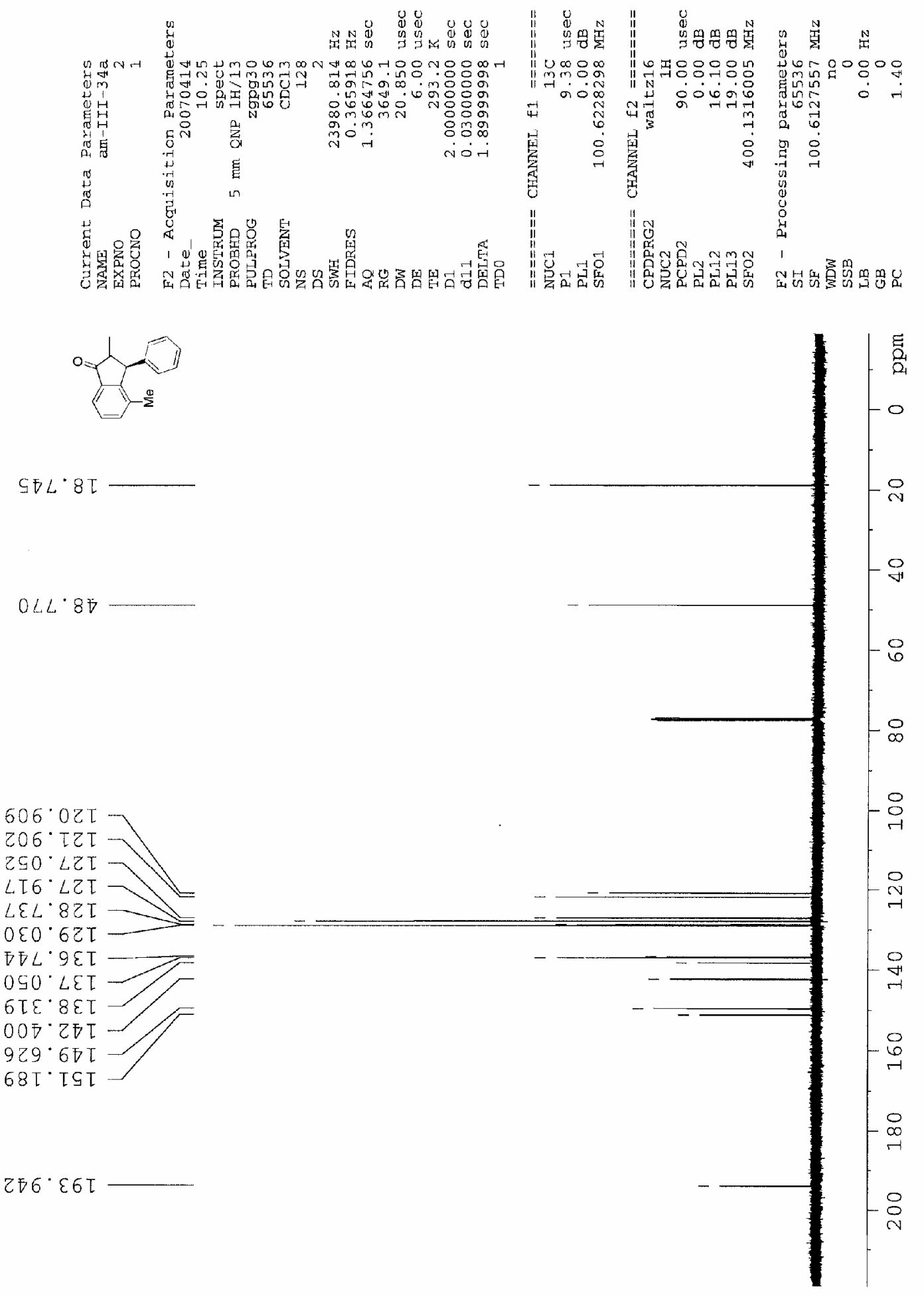

$57 L \cdot 8 I$

$O L L 8{ }^{\circ}$

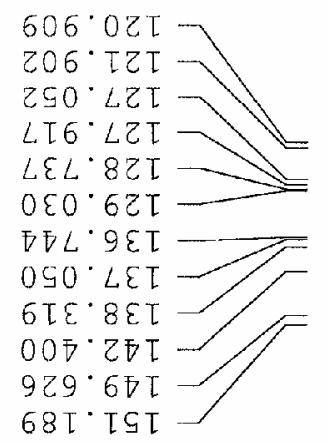

$2 \nabla 6^{\circ} \varepsilon 6 \tau$ 

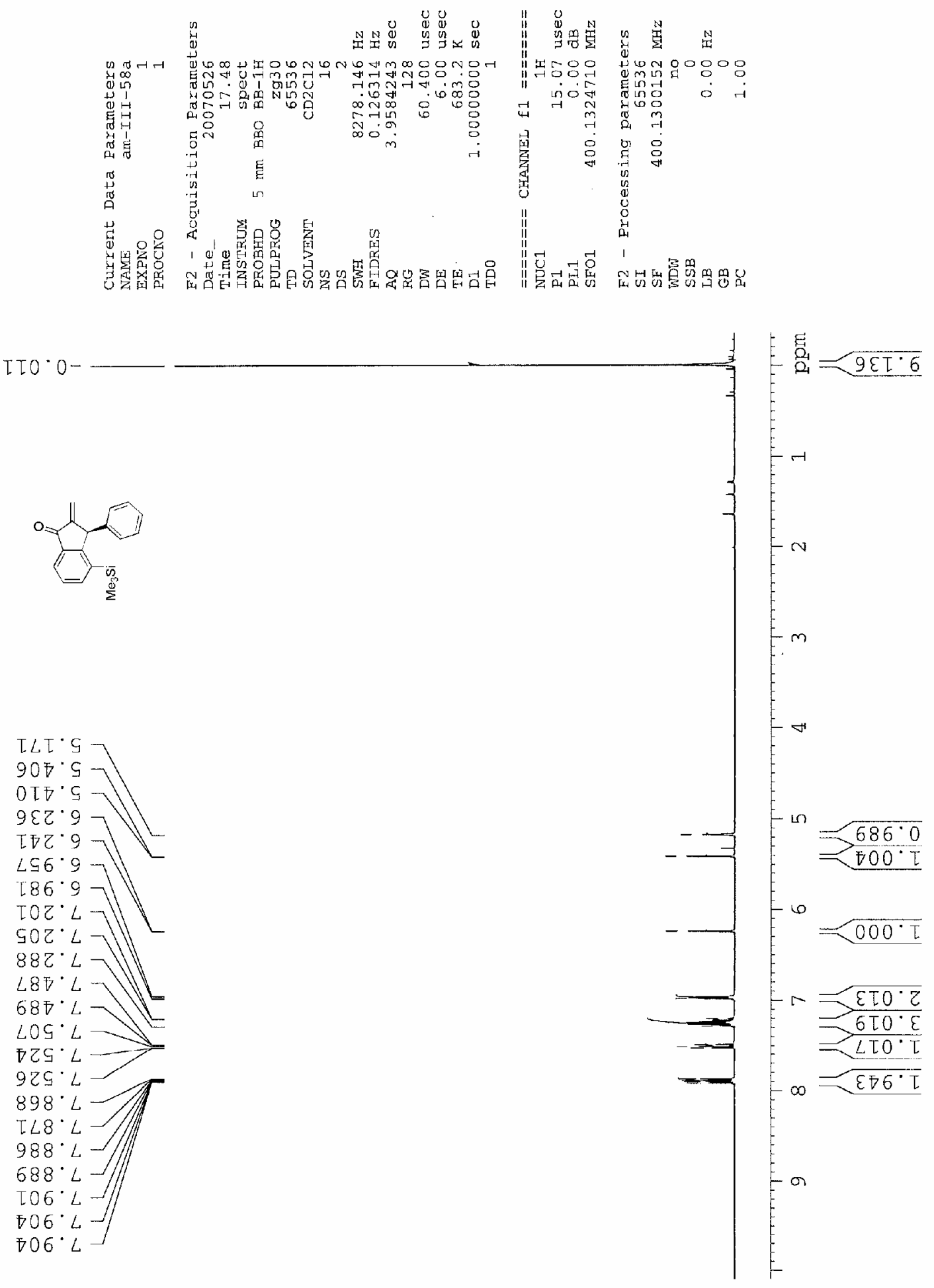

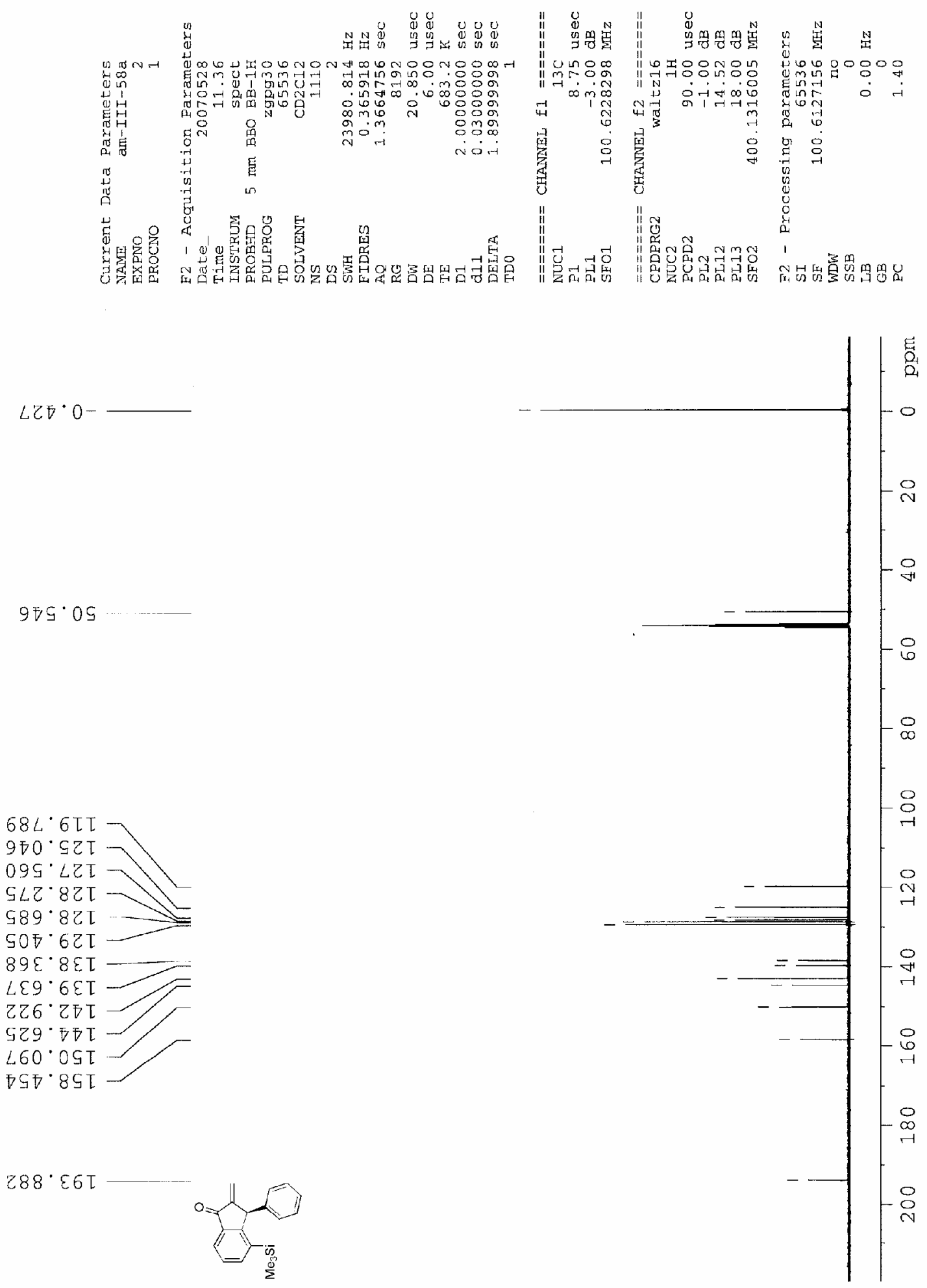

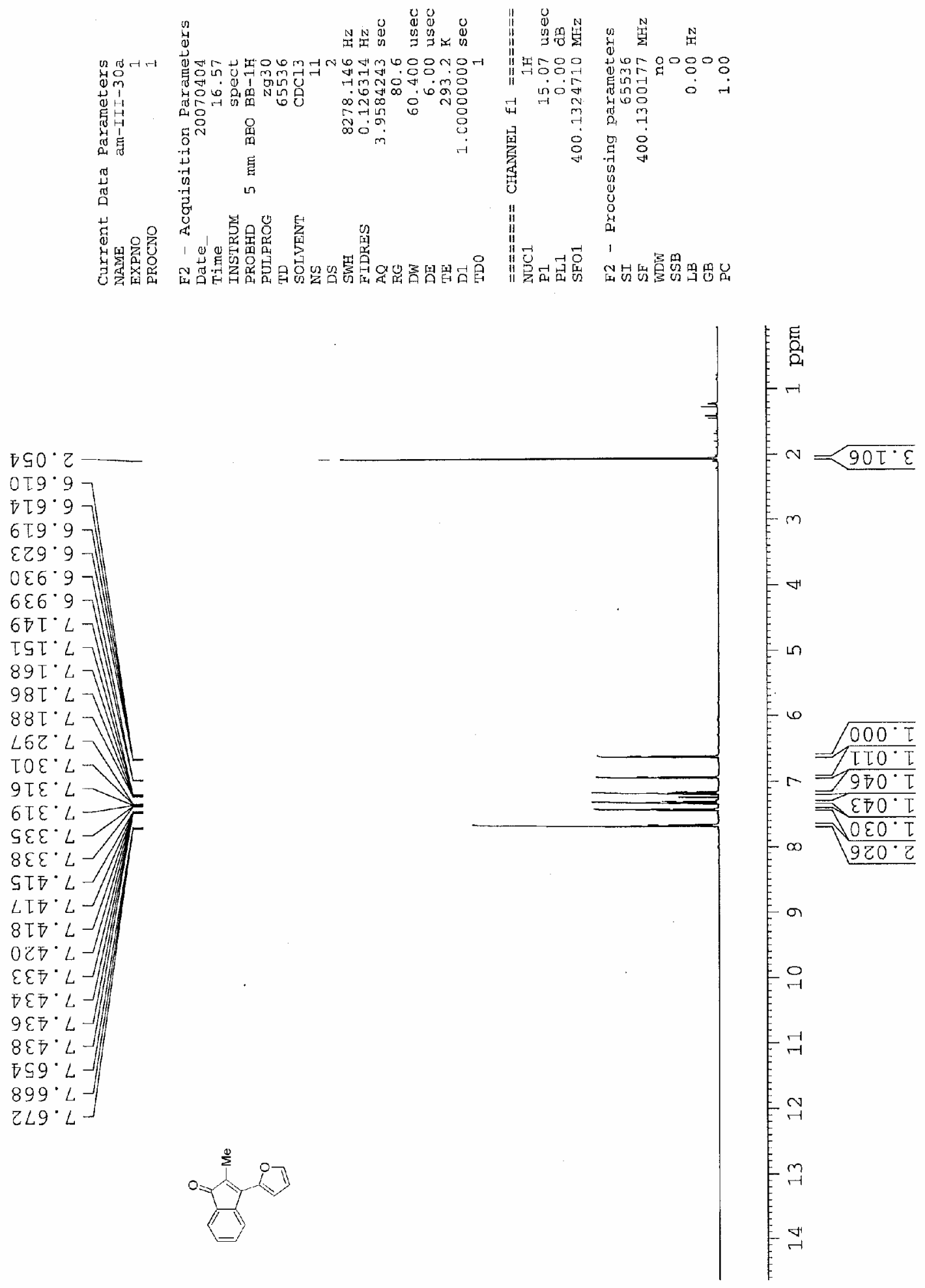

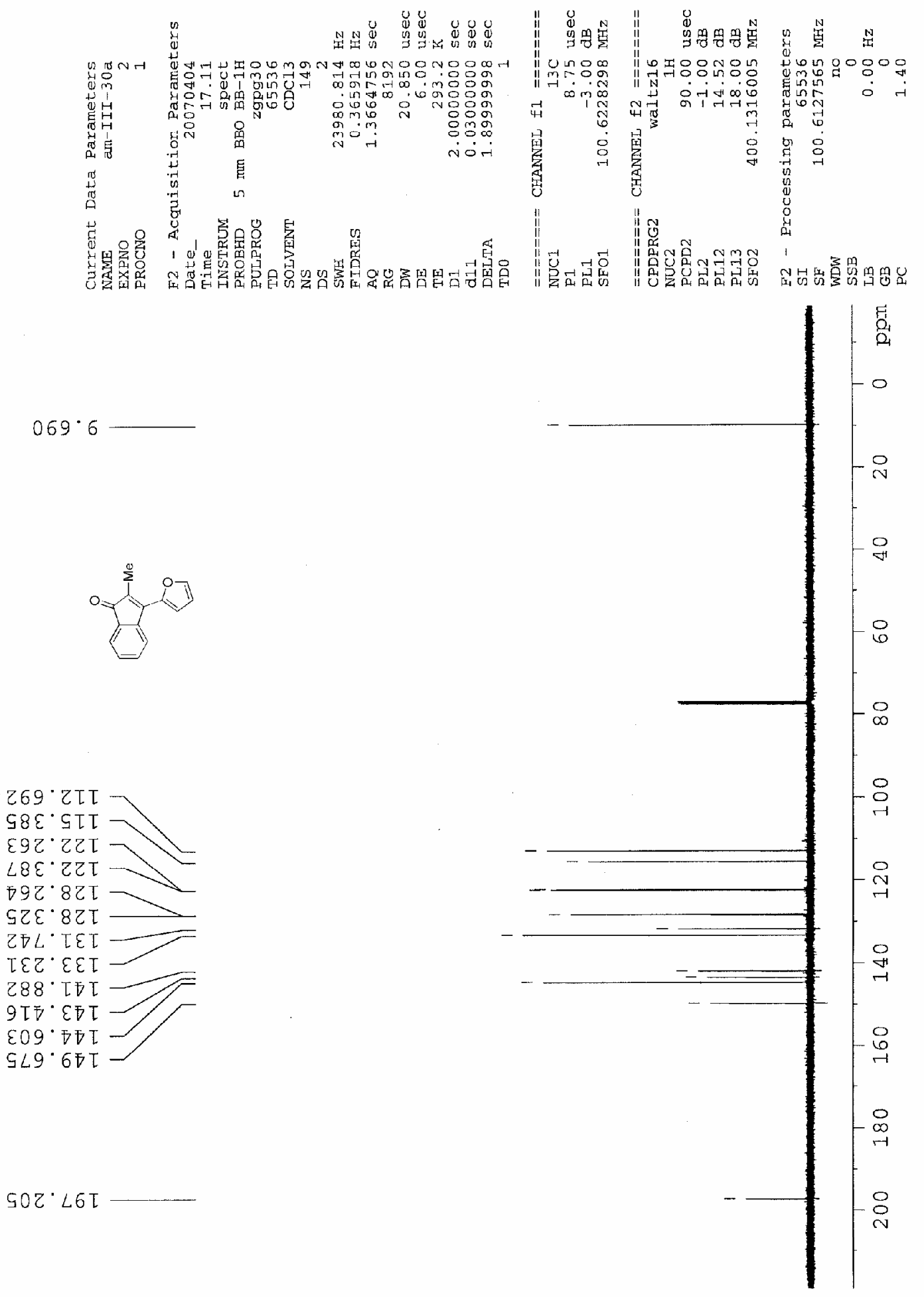

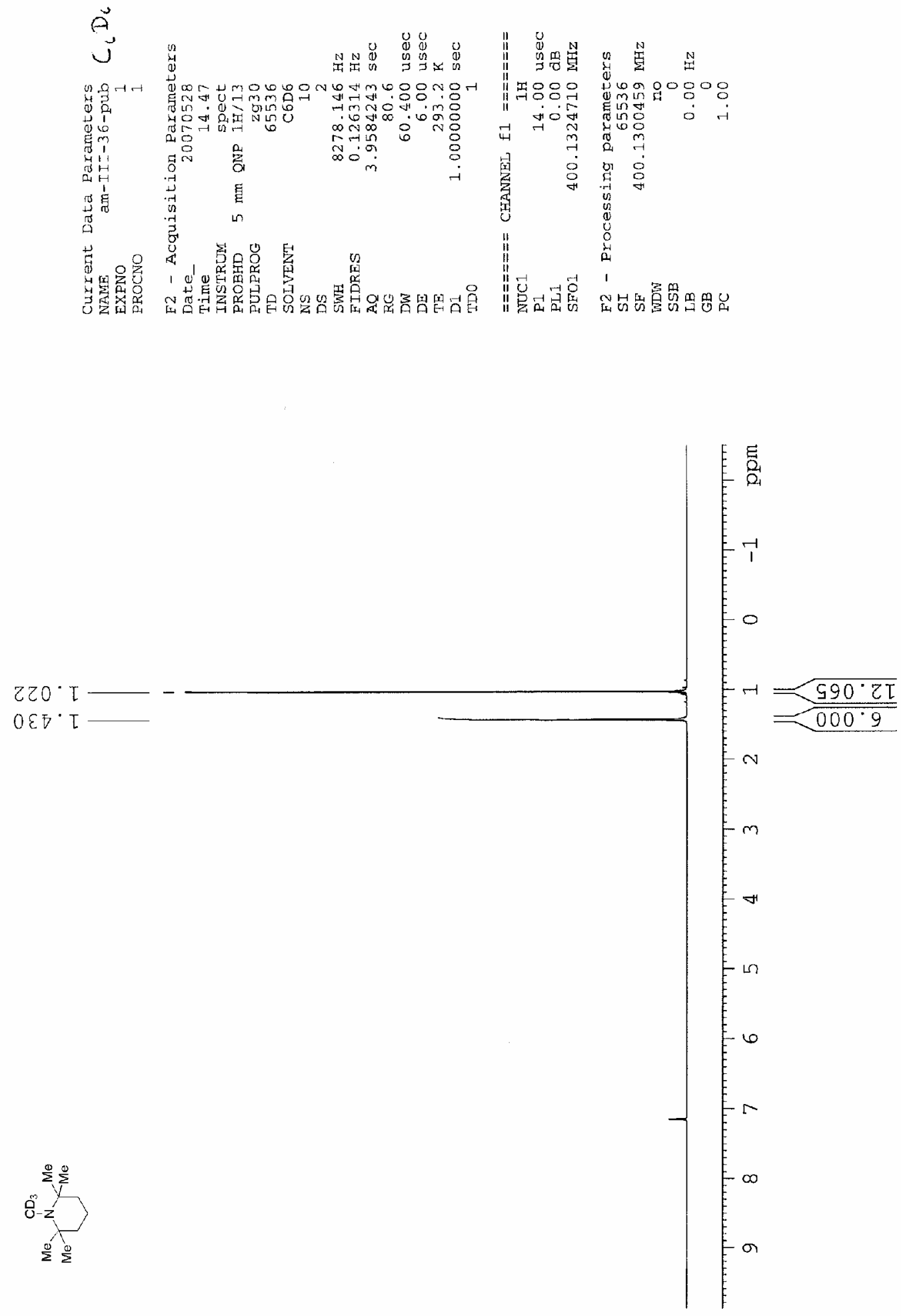

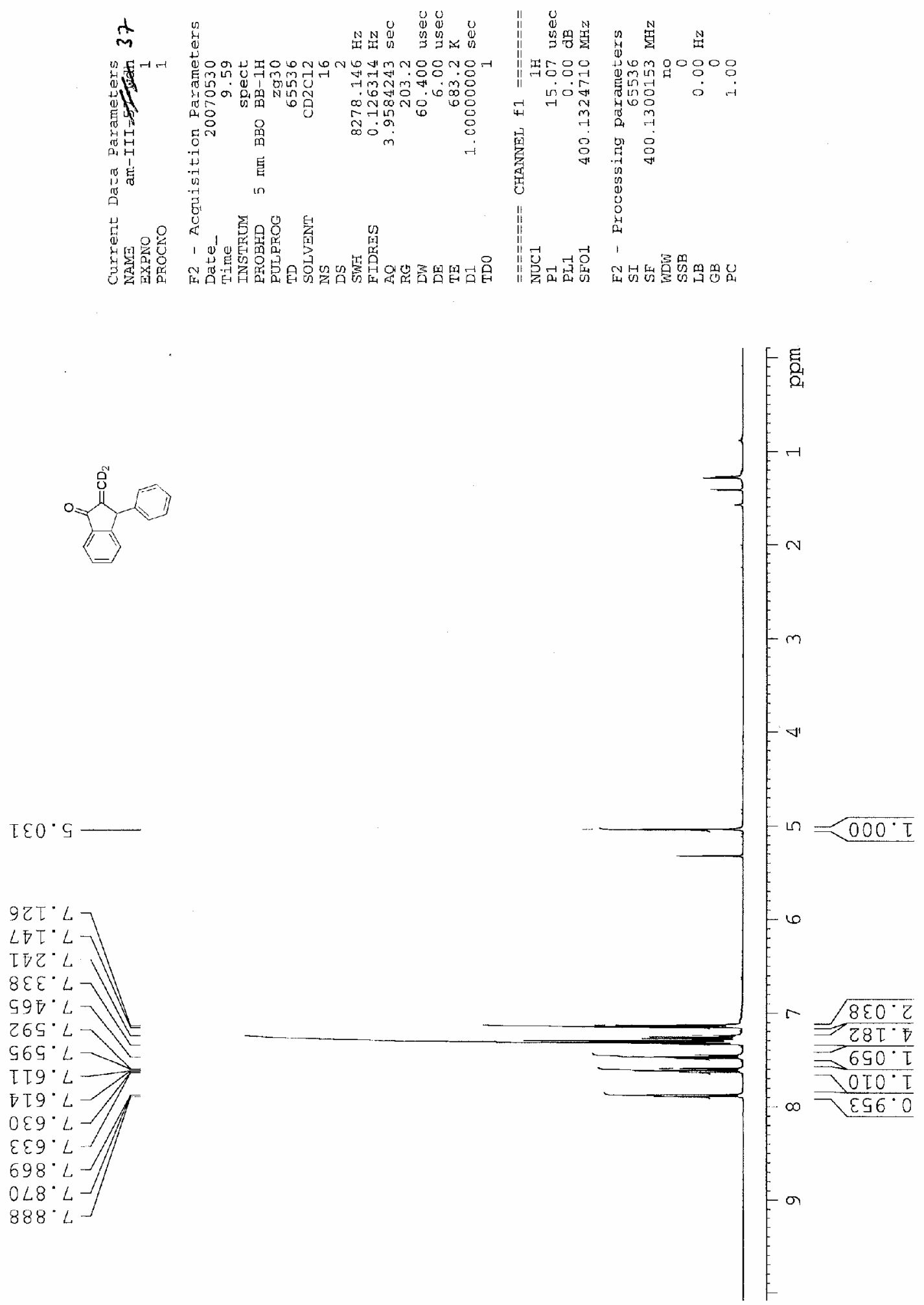

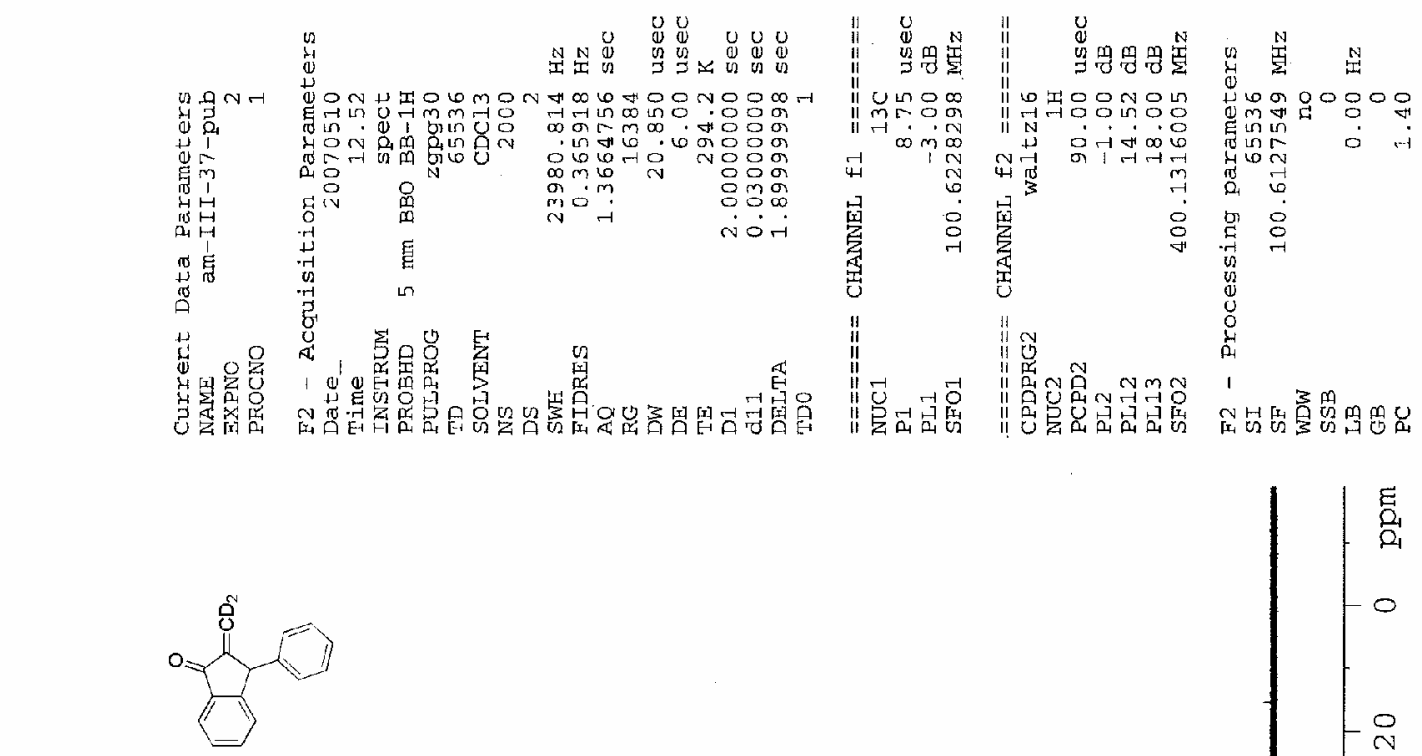

$\varepsilon 0 \tau^{*} \cdot 6 \bar{\square}$
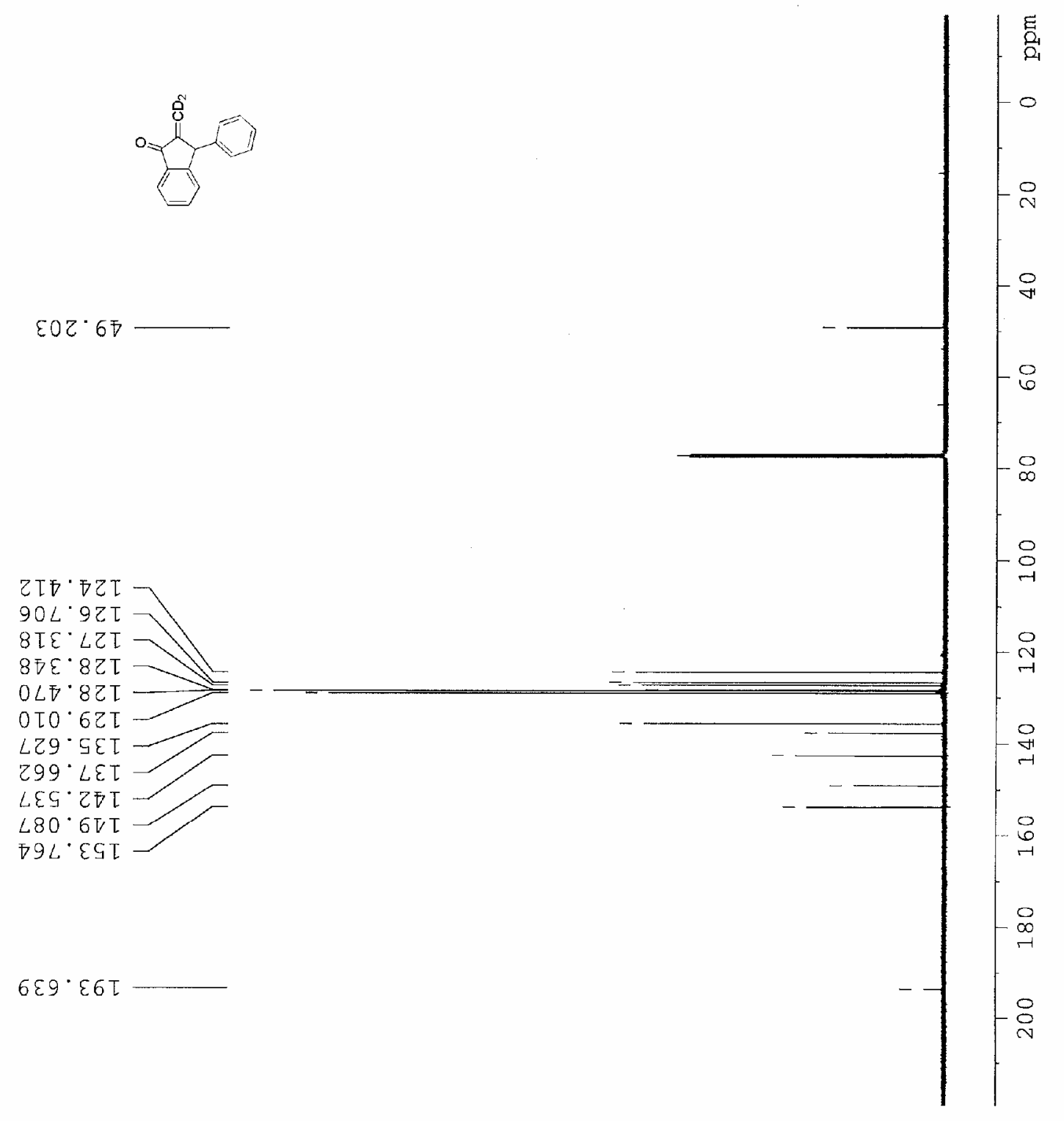

$6 \varepsilon 9 \cdot \varepsilon 6 \tau$ 

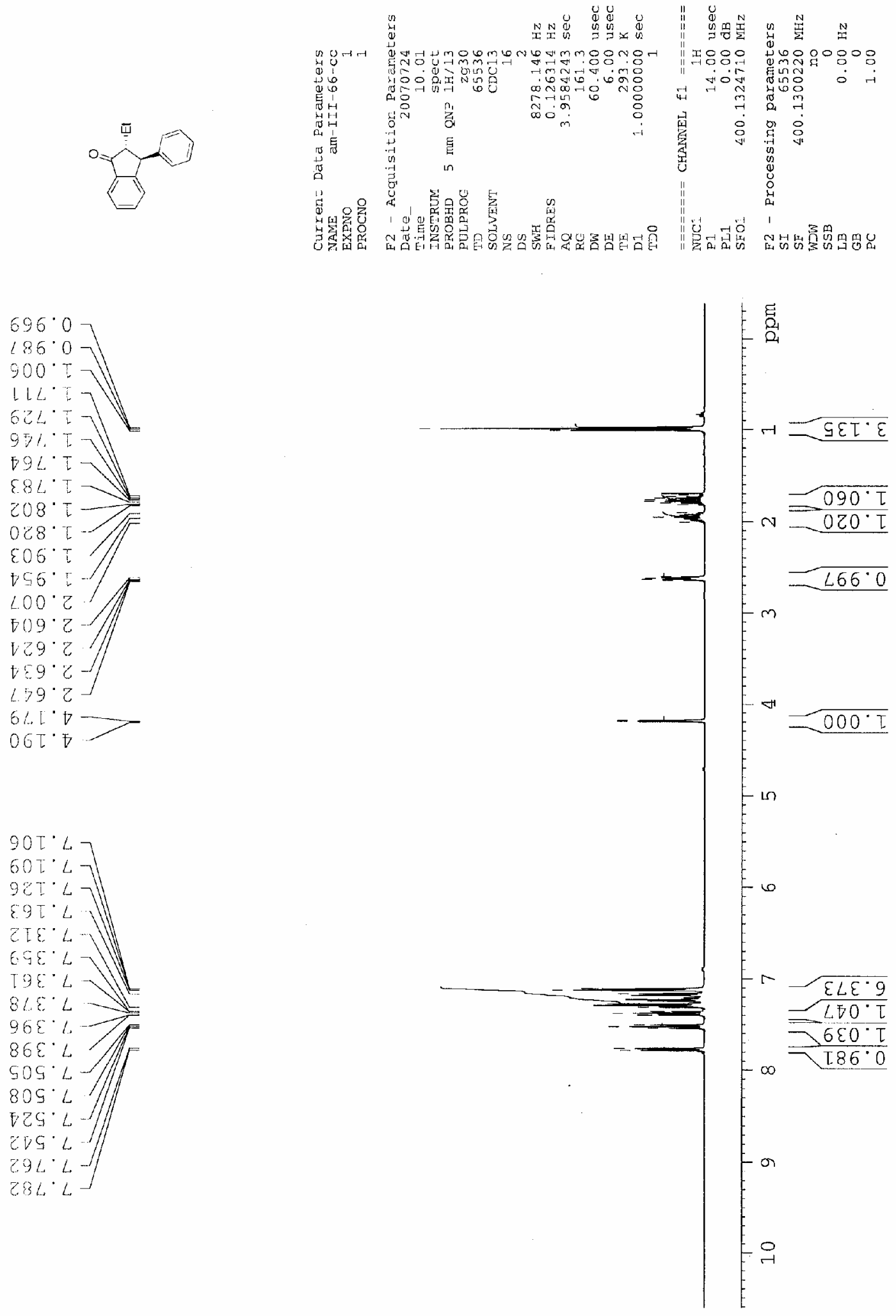


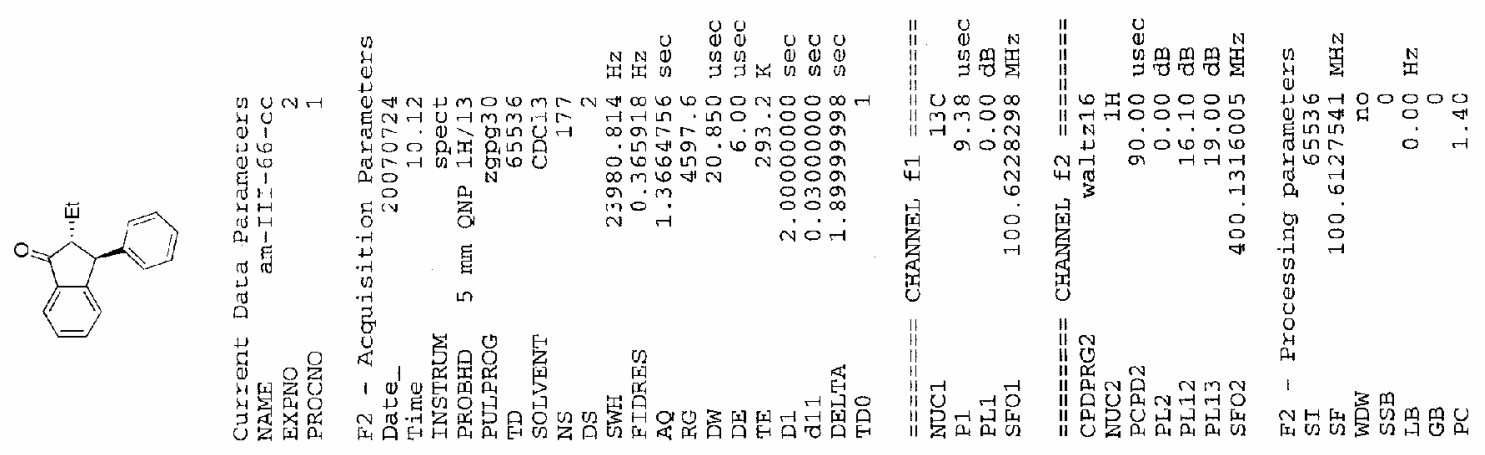

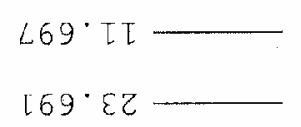

$80 \mathrm{C}^{\circ} \mathrm{TS}$

$9906 \mathrm{~s}$

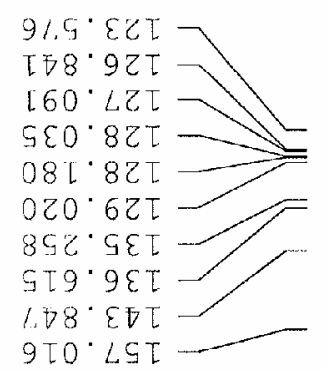

$[0 \tau \cdot 80 \tau$

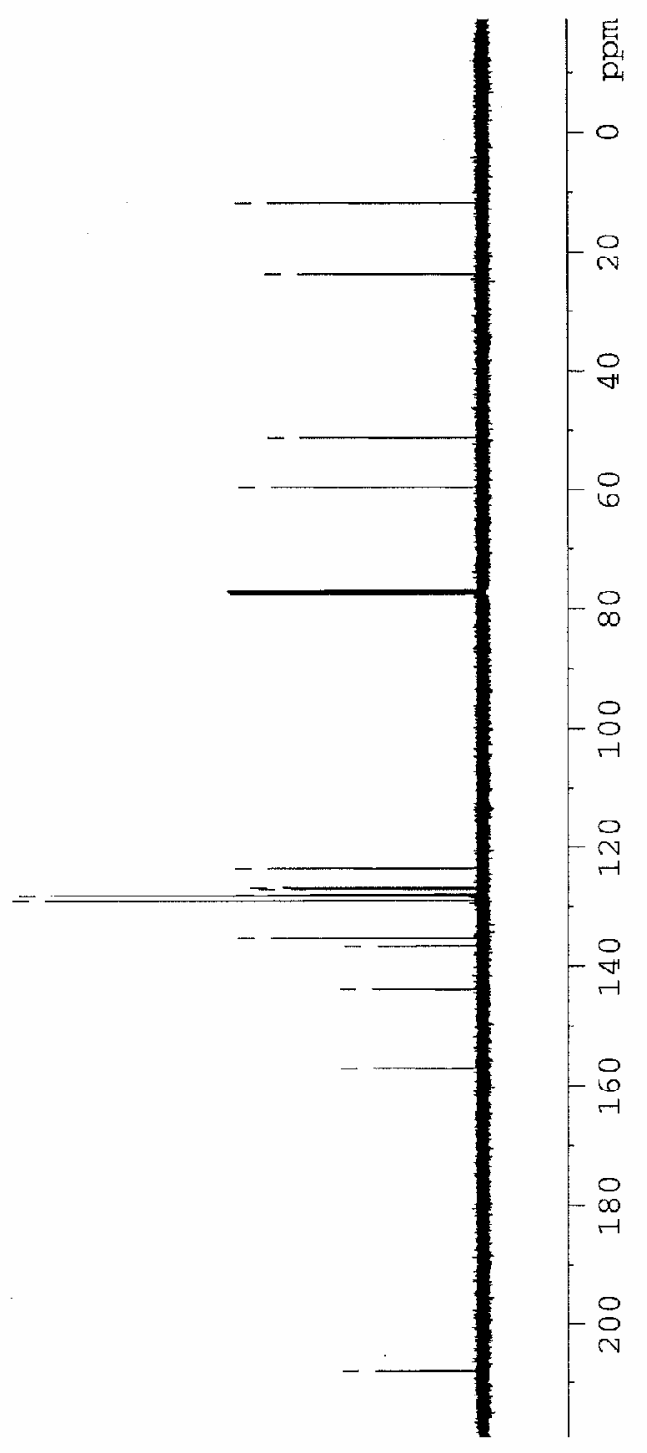



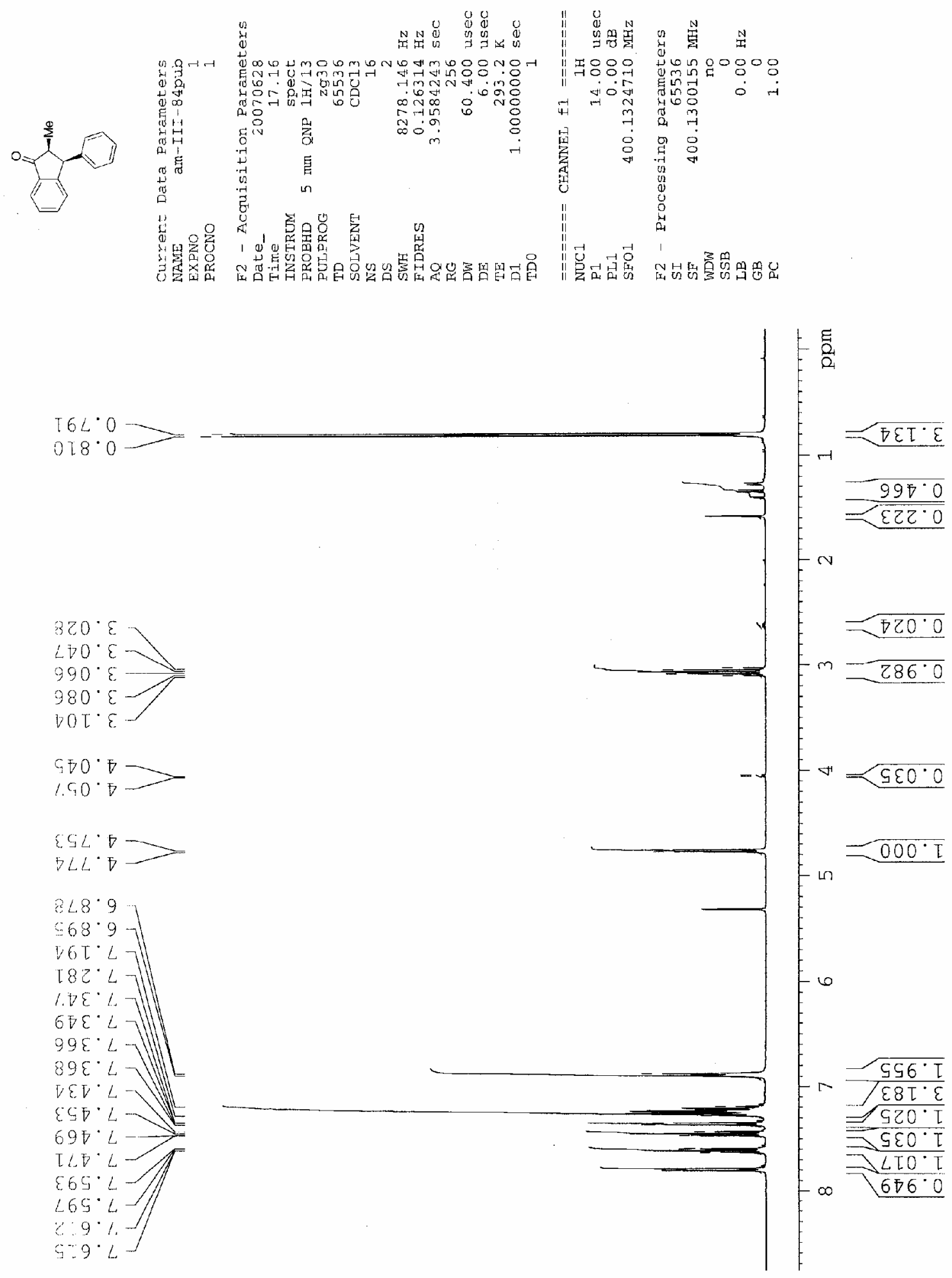

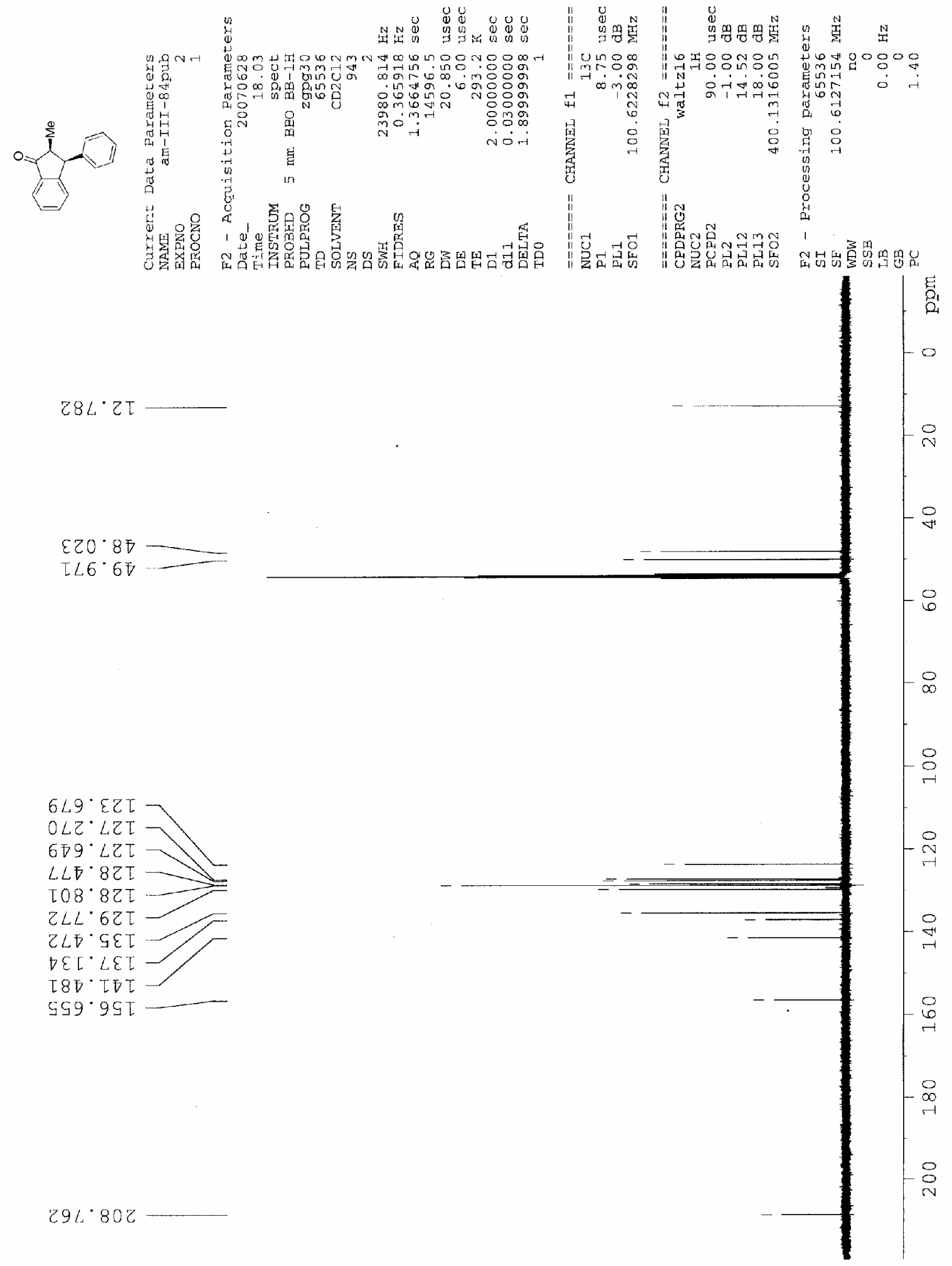
${ }^{\text {i }}$ Davey, W.; Gwilt, J. R. J. Chem. Soc. 1957, 1008.

${ }^{i i}$ Püschl, A.; Rudbeck, H. C.; Faldt, A.; Confante, A.; Kehler, J. Synthesis 2005, 2, 291.

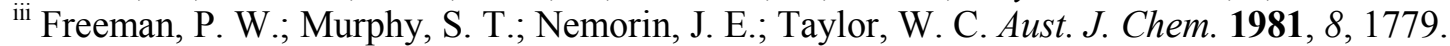

${ }^{\text {iv }}$ a) Kawamura, Y.; Maruyama, M.; Tokuoka, M.; Tsukayama, M. Synthesis 2002, 17, 2490. b) Liu, M.; Wilairat, P.; Croft, S. L.; Tan, A. L.-C.; Go, M.-L. Bioorg. Med. Chem. 2003, 11, 2729.

va) Hsieh, H.-K.; Tsao, L.-T.; Wang, J.-P.; Lin, C.-N. J. Pharm. Phramacol. 2000, 52, 163. b) Alcantara, A. R.; Marinas, J. M.; Sinisterra, J. V. Tetrahedron Lett. 1987, 28, 1515.

${ }^{v i}$ a) De, M.; Majumdar, D. P.; Kundu, N. G. J. Indian Chem. Soc. 1999, 76, 665. b) Alam, S J. Chem. Sciences 2004, 116, 325.

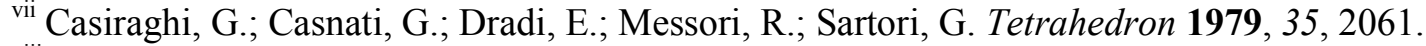

viii Nishide, K.; Ohsugi, S.-i.; Miyamoto, T.; Kumar, K.; Node, M. Monatsh. Chem. 2004, 135, 189.

${ }^{\text {ix } W o n, ~ S .-J . ; ~ L i u, ~ C .-T . ; ~ T s a o, ~ L .-T . ; ~ W e n g, ~ J .-R . ; ~ K o, ~ H .-H . ; ~ W a n g, ~ J .-P . ; ~ L i n, ~ C .-N . ~ E u r . ~ J . ~ M e d . ~ C h e m . ~ 2005, ~}$ $40,103$.

${ }^{x}$ a) Majumder, P. L.; Chatterjee, S.; Mukhoti, N. J Indian Chem. Soc. 2001, 78, 743. b) Old, K. B.; Main, L. J. Chem. Soc. Perkin Trans. 2 1982, 1309.

${ }^{x i}$ Donnelly, D. M. X.; Keenan, A. K.; Leahy, T.; Philbin, E. M.; Janzsó, G.; Kállay, F.; Koczar, I. Tetrahedron 1972, 28, 2545.

${ }^{x i i}$ Khan, H.; Zaman, A. Tetrahedron 1974, 30, 2811.

xiii The analytical/spectral properties are identical with those previously reported in the literature: a) Yun, J.; Buchwald, S. L. J. Org. Chem. 2000, 65, 767. b) Natori, Y.; Anada, M.; Nakamura, S.; Nambu, H.; Hashimoto, S. Heterocycles 2006, 70, 635.

xiv The synthesis of the racemic compound has been reported before: a) Seery, M. K.; Draper, S. M.; Kelly, J. M.; McCabe, T.; McMurry, T. B. H. Synthesis 2005, 3, 470. b) Pfeiffer, P.; Roos, H. H. J. Prakt. Chem. 1941, 159, 13. c) the synthesis of the enantiomerically pure compound has been reported before but no analytical evidence/properties are stated: Clark, W. M.; Kassick, A. J.; Plotkin, M. A.; Elridge, A. M.; Lantos, I. Org. Lett. 1999, $1,1839$.

${ }^{x v}$ The synthesis of the racemic compound has been reported before: a) Püschl, A.; Rudbeck, H. C.; Faldt, A.; Confante, A.; Kehler, J. Synthesis 2005, 2, 291. b) Barltrop, J. A.; Acheson, R. M.; Philpott, P. G.; MacPhee, K. E.; Hunt, J. S. J. Chem. Soc. 1956, 2928.

${ }^{x v i}$ The synthesis of the racemic compound has been reported before: a) Johnston, K. M.; Shotter, R. G. Tetrahedron 1974, 30, 4059. b) Rendy, R.; Zhang, Y.; McElrea, A.; Gomez, A.; Klumpp, D. A. J. Org. Chem. 2004, 69, 2340.

${ }_{\text {xvii }}$ The synthesis of the racemic compound has been reported before: a) Yu, H.; Kim, I. J.; Folk, J. E.; Tian, X.; Rothman, R.B.; Baumann, M. H.; Dersch, C. M.; Flippen-Anderson, J. L.; Parrish, D.; Jacobsen, A. E.; Rice, K. C. J. Med. Chem. 2004, 47, 2624. b) Bøgesø, K. P.; Christensen, A. V.; Hyttel, J.; Liljefors, T. J. Med. Chem. 1985, 28, 1817.

${ }^{x v i i i}$ The synthesis of the racemic compound has been reported before: Allen, J. M.; Johnston, K. M.; Jones, J. F.; Shotter, R. G. Tetrahedron 1977, 33, 2083.

${ }^{\text {xix }}$ The synthesis of the racemic compound has been reported before: a) Shotter, R. G.; Johnston, K. M.; Williams, H. J. Tetrahedron 1973, 29, 2163. b) v. Braun, J.; Manz, G.; Reinsch, E. Liebigs Ann. 1929, 468, 277.

${ }^{\mathrm{xx}}$ Barluenga, J.; Trabanco, A. A.; Flórez, J.; García-Granda, S.; Llorca, M.-A. J. Am. Chem. Soc. 1998, 120 , 12129.

${ }^{x x i}$ The synthesis of the racemic compound has been reported before: Koller, M.; Karpf, M.; Dreiding, A. S. Helv. Chim. Acta 1986, 69, 560.

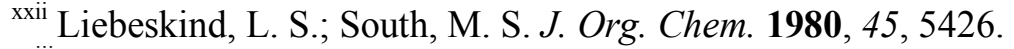

xxiii The synthesis of the racemic compound has been reported before: Hiscock, M.; Poter, G. B. J. Chem. Soc. B 1971, 1631. 